\title{
Chinese immigrant children in New Zealand early childhood centres
}

\author{
By \\ Karen Liang Guo (梁耿)
}

\begin{abstract}
A thesis
submitted to the Victoria University of Wellington in fulfillment of the requirements for the degree of Doctor of Philosophy in Education
\end{abstract}

Victoria University of Wellington

2010 


\begin{abstract}
This research investigated the learning experiences of Chinese immigrant children in New Zealand early childhood centres with the aim of describing educational implications for early childhood professionals.

A qualitative research approach was adopted using a multiple case study design. Eight Chinese immigrant children aged three to five years, their parents and their teachers participated in the study; the children were enrolled in six early childhood centres in a large urban area. Procedures of data gathering included child observations, and child, parent and teacher interviews. Data were analysed from phenomenological and sociocultural perspectives.
\end{abstract}

The children's learning experiences, particularly languages and interpersonal relationships, were discussed from the perspectives of sociocultural theories. The concept of a learning community contributed to the analysis which was also influenced by the notions of cultural diversity and multiculturalism.

A major theme of this research was the value of the culture of Chinese immigrant families to mediate the learning experiences of Chinese immigrant children in New Zealand early childhood centres. Familiar cultural tools and mediators provided important support for the children who were able to access them in their centres.

The children's intention to drive their own learning experiences was also a salient feature. Evidence was documented that illustrated how learning and development were mediated by fulfilment of feelings of belonging, as well as the children's commitment to cross cultural boundaries. The Chinese immigrant children were active drivers of their own learning and capably negotiated and created the relationships between their family culture and that of their early childhood centre. Specific strategies they adopted to construct their own learning experiences were found to be significant in explaining the emergence of hybrid cultural tools which mediated the children's evolving development of appropriate repertoires of practice in their early childhood centres. 
This thesis contributes to the body of sociocultural research via the examination of children's creation of intercultural learning possibilities. It provides early childhood teachers with insights regarding how to enhance pedagogical policies, values and practices to more closely align with sociocultural frameworks, concepts of learning communities and cultural diversity. It is important that diverse cultural relations are appropriately established in the early childhood centres so that immigrant children can move between different cultures in order to generate a useful intercultural way of being for themselves. 


\section{Acknowledgements}

I wish to thank the many people who have supported me to bring this thesis to fruition.

Associate Professors Carmen Dalli and Val Podmore's scholarly supervision has been excellent and I would not have asked for anything better. The undertaking of this research has been a long and intense endeavour and I thank them for walking alongside me to its completion. I thank Val for retaining me as a student when she semi-retired, and Carmen for the research grant applications, the pleasant meals and water view accommodations at her home, the airport collections, drop-offs, as well as all the opportunities that she and Val arranged to communicate with me. My supervisors' understanding of the difficulties due to my work commitments and my living outside Wellington and their support to overcome them are much appreciated.

Thanks to all my colleagues and friends at the Unitec Institute of Technology for the opportunities to talk about my thesis. I am extremely appreciative of Associate Professor Jenny Ritchie for her time and feedback. Discussions with Jenny about culture, diversity and multiculturalism were invaluable at the later stages of writing up this research. Help and discussion with Professor Carol Cardno about the research methodology and my 'thesis' are also appreciated. Thanks also to Robyn Reid and Maggie Haynes for their invaluable input in editing this work. Caroline Malthus is appreciated as well for assisting me with the writing structures of some chapters. I also acknowledge the management people at the Unitec Institute of Technology, Professor Carol Cardno, Helen Aitken and Kiri Gould who provided me with time and space to immerse myself in writing whenever possible.

My heartfelt thanks are also to my family for being unquestioningly supportive throughout my education career. I owe a debt of gratitude especially to my mother inlaw, who undertook so many family duties in my absence. XieXie mama! My two children, Yinuo and Jialiang also deserve a special mention. Yinuo developed herself into an outstanding teenager both academically and socially when mum was not always there for her. She also helped me draw the pictures of the stories I used with the children in this research. Jialiang was conceived exactly at the same time this research was proposed and he started school when the thesis was submitted. So, Jialiang has shared 
this research journey with me in a remarkable way. His early childhood years were precisely my $\mathrm{PhD}$ years. The inspiration from this special Chinese immigrant child has been immensely valuable to my understanding of the children in this study. Thanks are also due to my husband for being endlessly interested and encouraging of my professional development. I am also lucky to have my parents' trust, pride and confidence in me as I was travelling on this journey.

It is with much gratitude that I recognise the assistance of the Unitec Institute of Technology for funding the study fees, and the Victoria University of Wellington for awarding me the research grants in 2008 and 2009, and a PhD submission scholarship in 2010.

Grateful appreciation goes to Andrea Godfree who provided valuable assistance with the final formatting.

Finally I wish to thank all the children, teachers and families in the early childhood centres for their interest and participation. 


\section{Table of Contents}

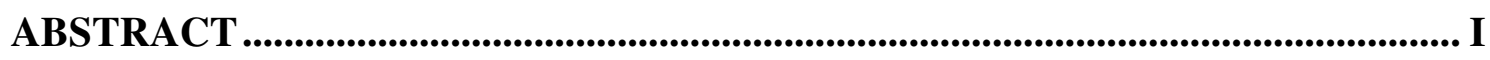

ACKNOWLEDGEMENTS.................................................................................................. III

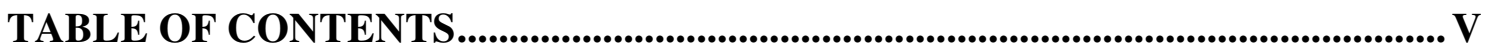

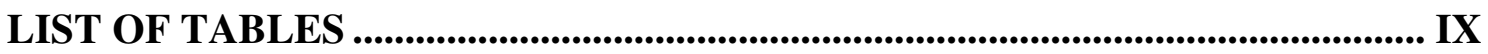

LIST OF FIGURES ............................................................................................................................. IX

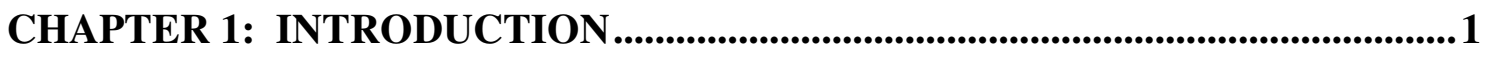

$\begin{array}{lll}1.0 & \text { Introduction } & 1\end{array}$

1.1 Background 1

1.1.1 Chinese immigrant children and families in New Zealand .............................................. 1

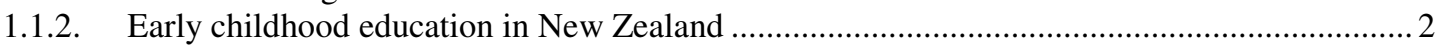

$\begin{array}{llr}1.2 & \text { Rationale } & 6\end{array}$

$\begin{array}{llr}1.3 & \text { Research questions } & 8\end{array}$

1.4 Researcher's statement 9

$\begin{array}{lll}1.5 & \text { Overview of the research } & 11\end{array}$

$\begin{array}{lll}1.6 & \text { Outline of the thesis } & 12\end{array}$

$\begin{array}{lll}1.7 & \text { Chapter summary } & 13\end{array}$

CHAPTER 2: THEORETICAL UNDERPINNINGS .......................................14

$\begin{array}{lll}2.0 & \text { Introduction } & 14\end{array}$

2.1 Sociocultural origins of learning and development $\quad \mathbf{1 5}$

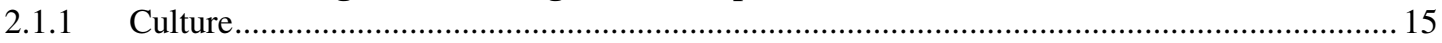

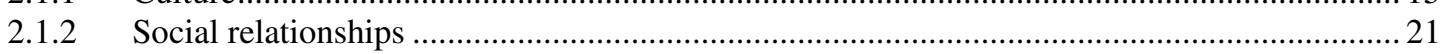

2.2 Learning as guided transformation of participation in families and in cultural
communities

2.2.1 Dealing with cultural variations ...................................................................................... 28

2.3 Concepts relating to multiculturalism and diverse cultural relations 29

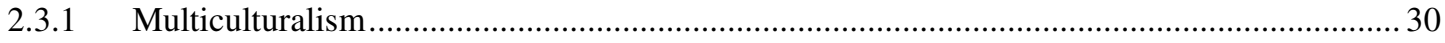

2.3.2 Cultural diversity and the difficulties in implementing cultural diversity .................................. 31

$\begin{array}{lll}2.4 & \text { Learning communities } & 33\end{array}$

$\begin{array}{lll}2.5 & \text { Chapter summary } & 34\end{array}$ 
$\begin{array}{lll}\text { 3.0 } & \text { Introduction } & 37\end{array}$

3.1 Children in Chinese immigrant families 38

3.1.1 Traditional Chinese cultural orientation to childrearing............................................................ 38

3.1.2 Immigration life of Chinese immigrant parents and children................................................ 40

3.2 Chinese immigrant children in non-Chinese speaking early childhood settings 46

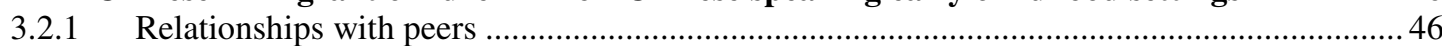

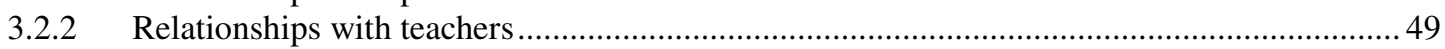

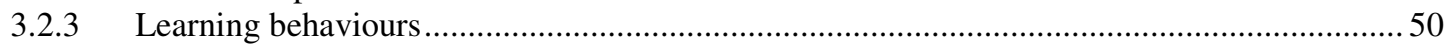

3.2.4 Second language learning and development ……….............................................................51

3.3 Working with immigrant children: The role of teachers $\quad 58$

3.3.1 Understanding and positively responding to differences ....................................................59

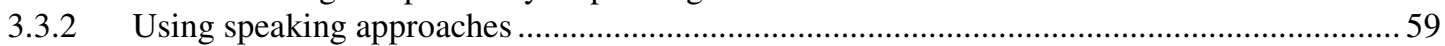

3.3.3 Supporting children's development in a new language and culture ............................................... 60

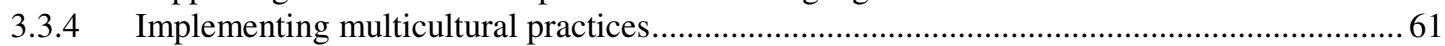

3.3.5 Developing partnership relationships with immigrant parents ................................................ 63

$\begin{array}{lll}3.4 & \text { Chapter summary } & 63\end{array}$

CHAPTER 4: RESEARCH METHODOLOGY AND RESEARCH DESIGN ......65

$\begin{array}{lll}4.0 & \text { Introduction } & 65\end{array}$

4.1 Methodological choice: Qualitative research $\quad 65$

$\begin{array}{lll}4.2 & \text { Position of inquiry: Phenomenology }\end{array}$

$\begin{array}{lll}4.3 & \text { Research practice for making inquiry: Multiple case study } & 67\end{array}$

$\begin{array}{lll}4.4 & \text { Methods of inquiry } & \mathbf{7 0}\end{array}$

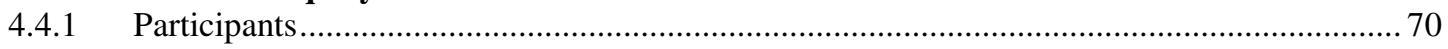

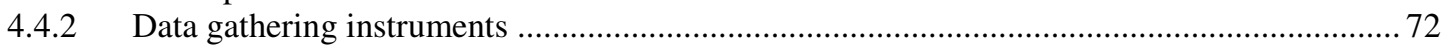

$\begin{array}{lll}4.5 & \text { Ethical approval } & \mathbf{7 6}\end{array}$

4.6 Data gathering procedures $\quad 77$

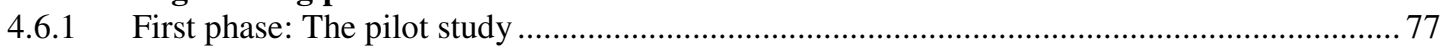

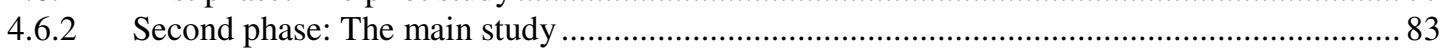

$\begin{array}{lll}4.7 & \text { Research rigour } & 92\end{array}$

$\begin{array}{lll}4.8 & \text { Field work reflection } & 94\end{array}$

$\begin{array}{lll}4.9 & \text { Chapter summary } & 98\end{array}$

CHAPTER 5: CHINESE IMMIGRANT FAMILIES AND NEW ZEALAND

EARLY CHILDHOOD CENTRES: THE CHILDREN IN CONTEXT .................100

$\begin{array}{lll}5.0 & \text { Introduction } & 100\end{array}$

$\begin{array}{lll}5.1 & \text { Introducing the children } & 102\end{array}$ 
5.2 New Zealand EC teachers' beliefs and practices about working with Chinese immigrant children

5.2.1 Attending to the children

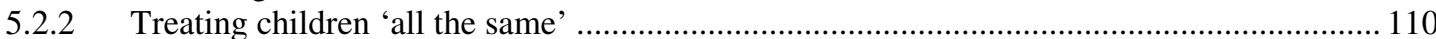

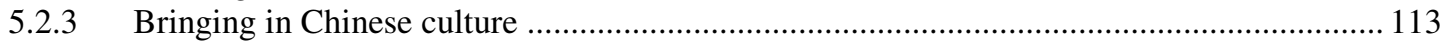

5.3 Chinese immigrant parents' beliefs and practices about child rearing and early educati 118

5.3.1 Serious learning only happens at home.....

5.3.2 Embracing the mainstream culture of the centres .......................................................... 121

5.3.3 Holding back their opinions from 'Kiwi' teachers ........................................................ 122

5.3.4 Communicating with Chinese teachers ........................................................................ 126

5.4 The children's perspectives of learning and developing in two different cultural communiti 128

5.4.1 Learning happens at home

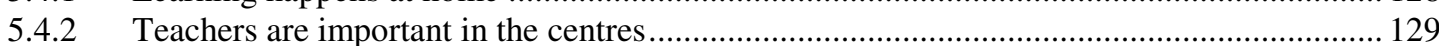

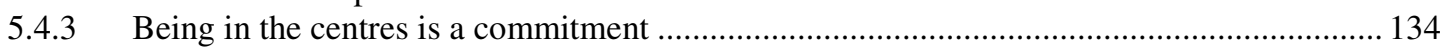

P.4.4 Parents are passers-by in the centres ..................................................................... 136

$5.5 \quad$ Chapter summary

CHAPTER 6: CHINESE AND ENGLISH: LANGUAGES AS A TOOL TO ACT UPON THE WORLD ..........................................................................................................141

$\begin{array}{llr}\text { 6.0 Introduction } & 141\end{array}$

6.1 Code switching between English and Chinese 142

$\begin{array}{lll}\text { 6.2 Experiencing the Chinese language } & 151\end{array}$

6.2.1 Seeking a sense of being together and belonging with Chinese speaking people ................ 151

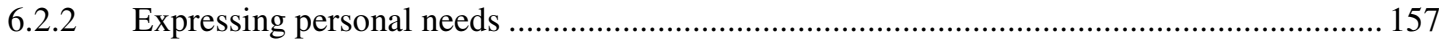

6.2.3 Mediating thinking and guiding behaviours.......................................................... 161

$\begin{array}{lll}6.3 & \text { Experiencing the English language } & 164\end{array}$

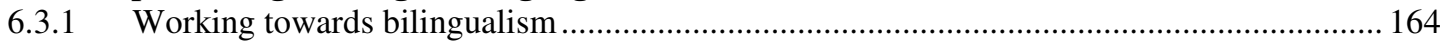

$\begin{array}{llr}\text { 6.4 Limited usage of English } & 179\end{array}$

$\begin{array}{llr}\text { 6.5 Chapter summary } & 182\end{array}$

\section{CHAPTER 7: CHINESE AND ENGLISH: PEER RELATIONSHIPS AS A}

TOOL TO MEDIATE LEARNING ..........................................................................186

$\begin{array}{llr}\text { 7.0 Introduction } & 186\end{array}$

$\begin{array}{llr}7.1 & \text { Relating to Chinese peers } & 188\end{array}$

7.1.1 Speaking Chinese and being Chinese as the main criterion for friendship ....................... 188

7.1.2 Obtaining a sense of belonging and togetherness as a small group within the larger

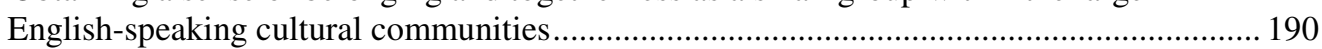

7.2 Relating to English-speaking peers 206

7.2.1 Learning with English-speaking peers was a desirable but complex experience ................206

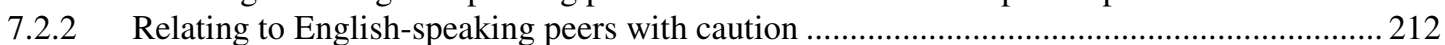

7.2.3 Aggressive reactions to peer conflicts...................................................................... 215

7.3 Chapter summary 


\section{CHAPTER 8: DRAWING THE FINDINGS TOGETHER AND LOOKING}

FORWARD

$\begin{array}{llr}\text { 8.0 Introduction } & 222\end{array}$

8.1 Drawing together: Conceptualizing the children's learning experiences 222

8.1.1 Learning experiences of Chinese immigrant children drew on the application of cultural tools of Chinese immigrant families and those of New Zealand early childhood centres ..... 224

8.1.2 Learning experiences of the children were driven by children's drive to belong................ 230

8.2 Looking forward: Educational implications 235

8.2.1 Introducing a focus on Chinese language and Chinese immigrant family culture ................ 236

8.2.2 Supporting the children's experiences with English-speaking peers ............................... 239

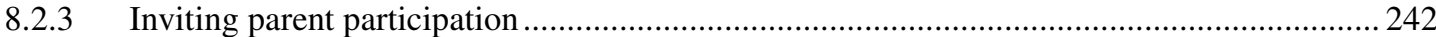

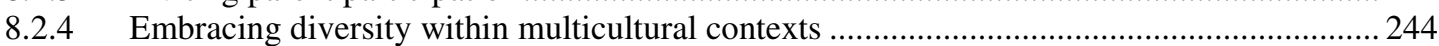

8.2.5 Developing teachers' professional strategies and relevant educational policies .................. 246

$\begin{array}{llr}\text { 8.3 Chapter summary } & 249\end{array}$

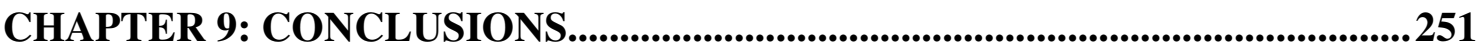

$\begin{array}{llr}9.0 & \text { Introduction } & 251\end{array}$

9.1 Key findings $\quad 252$

$\begin{array}{llr}9.2 & \text { Contribution to theory and knowledge } & 253\end{array}$

$\begin{array}{lll}9.3 & \text { Research limitations } & 256\end{array}$

$\begin{array}{llr}9.4 & \text { Future research } & 258\end{array}$

$\begin{array}{llr}9.5 & \text { Final remarks } & 260\end{array}$

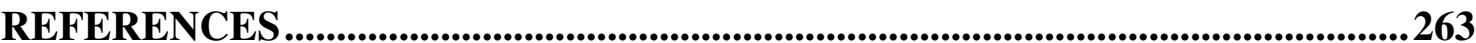

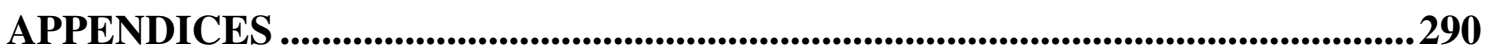

Appendix 1 Number of enrolments in early childhood education by ethnicity (Ministry of Education, 2008a)

$\begin{array}{llr}\text { Appendix } 2 & \text { Teacher participant information sheet } & 292\end{array}$

$\begin{array}{llr}\text { Appendix } 3 & \text { Parent participant information sheet } & 294\end{array}$

$\begin{array}{llr}\text { Appendix } 4 & \text { Consent forms for teachers and parents } & 295\end{array}$

Appendix $5 \quad$ Children's assent form 296

$\begin{array}{llr}\text { Appendix } 6 & \text { Children's interview schedule } & 297\end{array}$

$\begin{array}{llr}\text { Appendix } 7 & \text { Parent's interview schedule } & 300\end{array}$

$\begin{array}{llr}\text { Appendix } 8 & \text { Teachers' interview schedule } & 303\end{array}$

$\begin{array}{lll}\text { Appencix } 9 & \text { Teachers and parents' interview transcript feedback } & 305\end{array}$

$\begin{array}{lll}\text { Appendix } 10 & 306\end{array}$ 


\section{List of Tables}

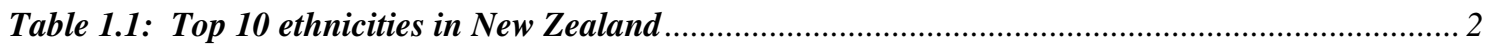

Table 4.1: Inquiry framework and methodological rationale .............................................................69

Table 4.2: Main study data gathering for each child ...................................................................... 86

Table 4.3: A line-by-line coding of a field note extract ............................................................. 88

Table 4.4: An example of organizing codes to develop a category .....................................................90

Table 5.1: Demographic and familial background of the children and their linguistic and peer

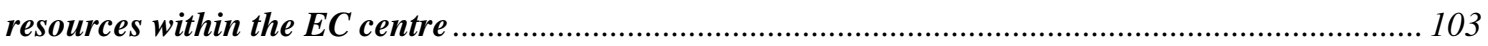

Table 5.2: Parents' morning arrivals with the children................................................................... 107

Table 6.1: The children's dual language usage in private situations............................................ 144

Table 6.2: Dual language usage in social contexts ......................................................................... 148

Table 6.3: Using Chinese to contact and seek togetherness with Chinese peers............................. 152

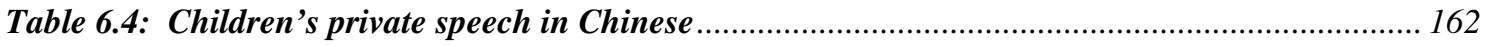

Table 6.5: The use of single words or formulaic speech............................................................... 171

Table 6.6: Asking Chinese speakers to help with English ...................................................... 174

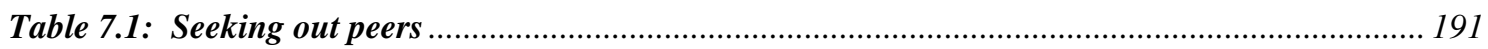

Table 7.2: Children's assertive communication to accomplish their own wishes ............................... 202

Table 7.3: Children's play experiences with peers during an-hour free play session....................... 208

Table 7.4: Categories of the children's relationships with English-speaking peers .......................... 211

\section{List of Figures}

Figure 5.1: Convergence of cultural practices and beliefs from the children's two cultural communities to guide children to settle in the centre

Figure 6.1: Chinese and English as common and simultaneous communicative devices to converge the two cultures.

Figure 6.2: The Chinese language bridges the two cultures. 164

Figure 7.1: Chinese immigrant family culture being kept apart from the culture of early childhoood centres. 195

Figure 7.2: Chinese-Chinese peer relationships to bridge the two cultures ..... 205

Figure 8.1: Representation of intercultural possibilities between Chinese immigrant family culture and the culture of New Zealand early childhood centres. 220

Figure 8.2: Child-driven hybrid cultural mediation. 234 


\section{Chapter 1: Introduction}

\subsection{Introduction}

This research investigated the learning experiences of a group of Chinese immigrant young children in early childhood (EC) centres in a New Zealand urban area with the aim of describing educational implications for teachers and families. It explored how children from Chinese immigrant families encountered the English language and group membership in their learning settings. Chinese parents' beliefs and practices about childrearing and early education, and those of New Zealand early childhood teachers about working with Chinese immigrant young children and their parents were investigated.

This chapter briefly discusses the background and rationale for the research. It also includes a summary of the research questions, the researcher's reflexive statement, as well as an overview and structure of the thesis.

\subsection{Background}

Because this research is focused on Chinese immigrant children entering New Zealand EC services, it is useful to begin with some demographic information about Chinese immigrant children and families in New Zealand and a brief outline of EC services in New Zealand.

\subsubsection{Chinese immigrant children and families in New Zealand}

Like elsewhere throughout the developed world, New Zealand has undergone a major population transformation in recent decades as a result of significant changes in immigration policies. These new policies aim to address economic demand by contributing overseas skills and finance into the New Zealand economy (Department of Labour, 2009). There has been an influx of immigrants, many of whom are people of Chinese ethnicity. The latest population census conducted in 2006 showed that Chinese was the fourth highest ethnicity group in New Zealand (Table 1.1). 
Table 1.1: Top 10 ethnicities in New Zealand

\section{Ethnicity group}

New Zealand European

Māori

New Zealander

Chinese

Samoan

Indian

Cook Islands Māori

Tongan

English

Korean

\section{Population count}

2,381,076

565,329

429,429

147,570

131,103

104,583

58,009

50,478

44,202

30,792

(Statistics New Zealand, 2006)

The increase in the number of Chinese residents contributes to the increasing enrolment of Chinese young children in New Zealand EC services. Statistical data from the Ministry of Education (2008a) suggests that from 2000 to 2007, the number of Chinese children enrolled in New Zealand EC services was the fourth highest among all ethnic groups. The enrolment of these children was steadily increasing over these years (Appendix 1).

\subsubsection{Early childhood education in New Zealand}

Early childhood education (ECE) in New Zealand occurs through a diverse range of services, including education and care centres (private or community-organised, full day or sessional), playcentres (sessional, parent collectives), kindergartens (sessional), Te Kohanga Reo (Maori immersion language nests), Pacific Island Language groups, and home-based services (a small group of children in a caregiver's home) (Ministry of Education, 2009a).

Despite the distinctive contexts across them, all licensed and chartered EC services in New Zealand are required to operate their programmes in line with Te Whäriki, the national ECE curriculum (Carter, 2008).

Te Whäriki (Ministry of Education, 1996) was released in the 1990s. Its development was a contextual response to the educational, social, cultural and political conditions in New Zealand at the time (Nuttall, 2005). The key focus of this curriculum is on bringing together a diverse EC community, and positioning the EC sector as made up of 
educational settings that provide education, care and socialization for children from birth to school age. The developmental perspective that promotes a 'child-centred', 'play-based' or 'informal' teaching approach, and the sociocultural emphasis on the importance of learning contexts are the binding theoretical structures of Te Whâriki (Cullen, 1996). According to Wu (2009), Te Whāriki is an eclectic mix of theoretical frameworks: "it endorses a developmental continuum; it also adopts a Vygotskian socio-cultural approach with an ecological standpoint" (p.73). The way that EC educators have traditionally gone about working with children involves the use of a child-centred approach (May, 2001). Te Whāriki aims to build on this tradition but create a sense that "the critical role of socially and culturally mediated learning" is also visible (Ministry of Education, p.9).

Consistent with the developmental and sociocultural perspectives, Te Whäriki supports the idea that children's learning experiences should be built on their major interests (Carr \& May, 2000), and are shared by teachers, parents and children in a collaborative participation process (Hedges, 2003). Frequently, children are encouraged to engage in child-initiated playful learning opportunities supported by their teachers (Anning, Cullen \& Fleer, 2004; Anning, Cullen \& Fleer, 2009; Hedges, 2007). In so doing, teachers are not expected to carry out didactic teaching but to be engaged with children in responsive and reciprocal interactions to encourage, support and extend children's learning from "the sum total of the experiences, activities and events, whether direct or indirect" (Ministry of Education, 1996, p.10). These teaching practices are premised on a principle that the holistic and total process of learning and development should be promoted in ECE (Anning et al., 2004; Anning et al., 2009). The holistic view also extends to valuing contributions from home, and learning experiences are shared between teachers and children's parents/families (Citizens Preschool and Nursery Centre of Innovation, 2008; Dalli, 1997; Grey, 2003; Mitchell, 2003). Adding to this is the practice in EC centres that recognises the importance of parents' contributions to teaching (MacNaughton, 2004; Ministry of Education, 2008b).

The changing context of early childhood education within which Te Whäriki was introduced and implemented also included other projects from the Ministry of Education to support early childhood teaching practice in New Zealand (Nuttall, 2005). One leading initiative was the release of learning stories, a narrative form of assessment 
based on the strands of Te Whäriki. The other was the development of Kei Tua o te Pae (Ministry of Education, 2004 \& 2005), a collection of early childhood exemplars that provide narrative cases of assessment to support EC teachers to work with children, parents and families on children's learning and assessment. The emphasis on narrative assessment in children's learning and development is an attempt to seek a match between the aims and goals of Te Whäriki and actual teaching practice with these guidelines (Haggerty, 1998). It is clearly stated in the Te Whäriki draft (1993) that, "assessment and evaluation should start from the children's needs and from the aims and goals of these [Te Whäriki] guidelines" (p.116).

For children from diverse language and cultural backgrounds, in addition to all the general educational philosophies and principles, the idea that has underpinned EC teaching practice is the embracing of cultural diversities:

The early childhood curriculum supports the cultural identity of all children, affirms and celebrates cultural differences, and aims to help children gain a positive awareness of their own and other cultures. Each early childhood education service should ensure that their programmes and resources are sensitive and responsive to the different cultures and heritages among the families of the children attending that service. (Ministry of Education, 1996, p.18)

According to Cullen (2003), an important theme in Te Whäriki is diversity. With the woven mat as its metaphor, this document aims to enable the weaving together of a curriculum that embraces diverse languages, cultures and socio-economic conditions of all children in New Zealand.

The most identifiable government initiative to support children from diverse language and cultural backgrounds is 'equity funding' for EC services to support children to learn English as a second language, or to provide learning programmes in a culture or language other than English. This support is planned to facilitate diversity, as well as minimise inconsistencies in children's learning due to their diverse backgrounds (Cullen, 2003).

Ngā Huaraki Arataki, the Strategic Plan for ECE, is a policy scheme that provides a framework for the government to take action to empower families and parents to be involved in their children's learning (Ministry of Education, 2002). It is an attempt to fulfill government commitment to ensure that "all families have access to quality 
education services that are responsive to their needs and those of their children" (p.9). A specific aim underpinning the implementation of the plan is that all aspects of a child's world are connected, including the language, culture and home learning experiences. The Strategic Plan was in the process of implementation when this research was carried out.

However, despite good intentions, there are discrepancies between practice and policy (Duncan, 2004; Nuttall, 2003). These arise because EC services are encouraged to implement programmes according to their particular sociocultural contexts and interests as illustrated in the following statement in Te Whāriki: "Each service will develop its own programmes to meet the needs of its children, their families, the specific setting, and the local community" (Ministry of Education, 1996, p.27). Teachers negotiate teaching practices in line with what they think are appropriate (Nuttall, 2003). As a result, teachers' different knowledge, experiences, training, and their views of children become the major issue in relation to the implementation of the EC policies (Cullen, 2003; Gibbons, 2005).

Similarly, practical applications to embrace cultural diversities in EC services face challenges. Cullen (2001) says, “phrases like 'accepting all cultures' do not always correspond to teaching practices; there can be a gap between curriculum discourse and reality which may not be easy to bridge" (p.6). Ritchie (2003) attributes this to the influence of teachers' own cultures. Ho, Holmes and Cooper (2004) describe teachers' limited understanding of other cultures as a barrier to their work with children from minority cultural backgrounds. The Education Review Office (2004) reported that 22 out of 53 EC services evaluated regarding their responsiveness to diverse cultures were making limited effort to cater for the cultures in their services. Due to a lack of practical guidance on the use of the equity funding for immigrant children, several years after it was introduced, Cullen (2003) expressed her concern about the effectiveness of this initiative. Although successful examples have been identified (Gerreiy, 2003; Glass, Baker, Ellis, Bernstone \& Hagan, 2008; Stewart-Mackenzie, 2007), some EC services were found to be in need of improving teaching approaches to diversity (Mitchell, 2008; Mitchell \& Hodgen, 2008). 
In summary, the attempts by the New Zealand government to support EC services to raise the educational achievement of young children demonstrate that the government recognises the importance of early learning experiences and the values of ECE. Despite the fact that there are discrepancies between policies and practices, the EC sector in New Zealand is provided with a pedagogical framework that privileges "holistic, strength and interest-based" learning and teaching (Cullen, 2008, p.10).

\subsection{Rationale}

This study was undertaken for several reasons.

The first reason is the scarcity of research about the early educational experiences of Chinese immigrant young children both in New Zealand and other countries. Although the increase in the number of Chinese immigrant children is significant, an extensive search of New Zealand literature located only one relevant study carried out in EC centres. This study considered the experiences of two Chinese immigrant toddlers and their parents (Chan, 2006). Most overseas research on Chinese immigrant children concerns school-aged children and commonly addresses two issues: intergenerational conflicts between parents and children due to the discrepancies in the acculturation levels; and psychiatric problems as a result of adaptive challenges during the settlement (Costigan \& Dokis, 2006; Lee \& Chen, 2000; Rao \& Yuen, 2001; Wu \& Chao, 2005; Zadeh, Geva \& Rogers, 2008). Most scholarly literature on young Chinese immigrant children is concerned with only one aspect of the learning experiences of these children, for example social behaviours or language (Chiang, 2003; Liu, 2006; Wang \& Pape, 2007), or providing insights to Western teachers about Chinese immigrant families' educational traditions and orientations (Ebbeck \& Gokhale, 2004; Feng, 1994; Huntsinger, Huntsinger, Ching \& Lee, 2000; Li, 2006). One study by Feng, Foo, Kretschmer, Prendeville and Elgas (2004) explored Chinese preschoolers' experiences with language and peer relationships in English-speaking settings, but not the overall learning experiences of the children.

In the research literature, studies regarding Chinese immigrant young children viewed them as one group of culturally and linguistically diverse children on a broad spectrum of other cultures (Barnard, 2003; Gonzalez-Mena \& Bhavnagri, 2000; Okagaki \& Diamond, 2000; Tabors, 1998; West, 2001). I consider these studies to be only partly 
relevant to one's understanding of Chinese immigrant children as they look at 'culturally and linguistically diverse' children as an entire unit and offer suggestions applicable to many groups but not specifically to Chinese immigrant children. In light of the uniqueness of Chinese culture, parenting styles and the children themselves, the most relevant approach to studying Chinese immigrant young children should be one that focuses exclusively on these children.

In terms of research into the experiences of local children settling in EC services, studies have shown a variety of challenges they confront (Dalli, 1998; Dalli, 1999; Dalli, 2000). Dalli reported, "starting childcare was a physically tiring experience which [children] needed to get used to" (1998, p.57). She talks of this experience for children as a challenging process that involves them separating from their mothers, developing trust with the people in the new environment, gaining familiarity with new routines, and "learning to fit in" (1999, p.361). If we add to these those specifically faced by immigrant young children, such as the difficulties of communication in an unknown language and of function in line with the unfamiliar cultural knowledge and practice, we begin to see the hurdles that immigrant children must overcome. Rogoff (2003) describes how children who are raised to be members of their culture could face many difficulties when trying to function in another cultural system. This gives an important justification for my research. Whilst there is evidence that immigrant young children encounter difficulties when settling in EC services (de Melendez \& Beck, 2007; Ryu, 2004; Tabors, 1998), none of these studies was conducted with Chinese immigrant young children. It is important that this study specifically focuses on these children and aims to investigate how they learn and develop in English-speaking EC centres.

Another reason for the choice of this research is the New Zealand government's policy and expectation for EC services to embrace cultures and the concept of diversity (Ministry of Education, 1996). If 92\% of teachers in New Zealand EC centres are of non-Asian ethnicity (Ministry of Education, 2008c), then it is reasonable to speculate that working with Chinese immigrant children could be challenging for some teachers, which highlights a need to identify insights that might be helpful for them.

A final reason for this study is my own background as a Chinese immigrant parent and as an EC professional, who trained and has worked in a number of EC institutions in 
New Zealand. I have been approached by both Chinese immigrant parents and New Zealand EC teachers regarding concerns and issues they faced as they worked with each other. Having a common language with both groups and the knowledge and experiences of the theories and practices used in both contexts makes me an appropriate person to conduct this research.

\subsection{Research questions}

This study is premised on the belief that children's learning and development are specifically influenced by their cultural contexts. It proposes that Chinese immigrant young children's learning experiences are grounded in the mainstream culture of New Zealand EC centres, as well as the culture of their families. I use the term 'culture' without an 's' here for the purpose of fluid writing. However, it is understood that a culture is never a single set of behaviours, beliefs and practices. Based on this thinking, while I will refer to 'Chinese immigrant family culture' and 'the culture of EC centres' in the singular in this thesis, I take the view that culture is multifaceted by nature.

The study recognises that the differences between two cultural communities in terms of raising and educating young children will provide Chinese immigrant children with distinct and different sets of cultural practices that they need to use to respond to opportunities in specific contexts of the EC settings (Rogoff, 2003; Vygotsky, 1978). Children's learning experiences can be unique to a certain culture in one situation or in a form of blending aspects of the two cultures in the other. Underlying the investigation of the children's learning experiences therefore will be an exploration of the 'coexistence' or 'relation' between the two cultural practices.

Given that language and interpersonal relationships are the main cultural components for learning and development (Vygotsky, 1978), it is envisaged that an understanding of the children's experiences with these will provide this research with valuable findings.

This research seeks insights that might be used to facilitate the learning and development of Chinese immigrant children in New Zealand EC centres through understanding the experiences of a small group of children. The input of Chinese immigrant parents and New Zealand EC teachers will be important in this because they 
play significant roles in Chinese immigrant children's lives and are able to provide valuable information about Chinese immigrant children's learning experiences.

Specifically the research explores the following questions:

1. How do Chinese immigrant children experience learning and development in New Zealand early childhood centres?

1.1. In what ways do Chinese immigrant children experience the English language?

1.2. How do Chinese immigrant children experience their learning as a member of a group in New Zealand early childhood centres?

2. How can Chinese immigrant children be supported towards a positive learning experience in New Zealand early childhood centres?

2.1. What are New Zealand early childhood teachers' beliefs and practices about working with Chinese young children and their parents?

2.2. What are Chinese parents' beliefs and practices about childrearing and early education?

\subsection{Researcher's statement}

There is a Chinese proverb that states that to know the road ahead, one must ask those coming back. Implicit in this proverb is a belief that the people who have walked along a road have knowledge, experience and opinions about it. This is a pertinent metaphor for understanding my journey as the researcher in this study. As a member of the Chinese cultural group and a mother of two children, I share a point of identification with the Chinese participants in this research. I had travelled on the road that this research explored and I had knowledge, experience and personal opinions about it. Therefore, as I present this report of my research journey, I feel that I must explain my own positions. As an insider in this research, I am likely to leave in it traces of my own voice.

I know from my own experience that the use of the term 'Chinese' is in many senses a confusing one. As with other 'categorizing' terms, such as 'boy', 'building', or 'flower' 
there is no clear definition of this term. In some cases, particularly in immigrant countries like New Zealand, Chinese embraces every person of Chinese descent (Zhang, 2010). There is, thus, a large diversity among Chinese. For a closer match to the research questions, in this research, I take a relatively specific definition for my use of the term Chinese, referring to it as people who immigrated to New Zealand from mainland China, Taiwan or Hong Kong within the decade in which the research began. Their children either came with them from the home country or were born in New Zealand. By this definition, I am an obvious insider to this research as I too immigrated to New Zealand within the decade of this research.

Literature suggests that research conducted in a community where the researcher possesses intimate knowledge of the research participants has advantages and shortcomings (Hellawell, 2006). Due to their familiarity with the participant culture, researchers can enjoy enhanced rapport with their research participants, and be privy to insider thoughts and information that may not be trusted to outsiders. On the side of shortcomings, the informed perspectives of the researcher may influence the data gathered. These different outcomes of insider research highlight the importance for me to reflect on my own beliefs and values, as well as my research methodology. In Chapter 4, I further reflect on my field work experience (see section 4.8).

I was born in mainland China in the early 1970s, a transitional time between Mao Zedong's communist influence and Deng Xiaoping's economic reforms. Socially and culturally, it was a time of significant cultural review in China. Many traditional beliefs, particularly those of Confucianism were re-emphasised. I started school at the age of five and a half. My whole schooling was a time of a great emphasis on academic achievements and the phrase ‘万般皆下品唯有读书高, meaning everything is subordinate to study success, permeated every class that I was in.

Upon completion of my study at a medical institute, I gave up my beginning career as a medical practitioner and immigrated to New Zealand with my family. In the years that I have been in New Zealand, I have seen myself continuing my academic pursuit in the same way as I did in China. Trying hard in study was also a principle that I promoted to my daughter, who was constantly under pressure to cope with many out-of-school 
learning activities. There is no doubt that what I learned and experienced in China has been influencing my values and practices as a person and a parent in New Zealand.

Having lived in New Zealand, I also experienced the process of adapting myself to fit into the social and cultural systems of this country. The opportunities of new education and work introduced me to many different approaches to life. I was surprised to see myself becoming a relaxed mother with my son, my second child, who had a much more playful childhood than his sister did. Although my son was introduced to academic experiences, such as counting or writing before he started school, my parenting priority with him was the development of his confidence and ability to socialise and solve problems.

It is against my life experiences both in China and in New Zealand that the focus of this research is set. These backgrounds have grounded my beliefs about children, approaches to life, and my actual practices. I acknowledge that my own experiences and knowledge influenced the present research. While this qualitative research "values [my] personal involvement and partiality ('subjectivity')" (Davidson \& Tolich, 2003, p.124), I have made every effort to rigorously keep my own experience out of the experiences of the participants (see section 4.8). Nevertheless, it is not the purpose of this research to produce generalizable findings, but rather to reveal a process of synthesizing the sociocultural elements pertinent to the learning experiences of the children in this study, illustrating them with examples, exposing their dynamics, and exploring implications for teaching in early childhood education and care settings.

\subsection{Overview of the research}

A qualitative multiple case study approach was adopted for the study. It aimed to describe the learning experiences of eight Chinese immigrant children in six EC centres in New Zealand. Sociocultural influences on learning and development, as proposed in sociocultural theories, were at the centre of my deliberations in this research. The research design was underpinned by a 'watching, listening and reflecting' technique. It involved child observations in the EC centres and interviews with the children, their parents and teachers about their perspectives of the children's experiences, and the parents' and teachers' own experiences when living or working with the children in the study. The research focused on understanding and interpreting each child's experience 
of various opportunities in the EC environment, people's construction of meanings around these experiences and their subsequent responses to these experiences. The explanation for this design is that knowing the Chinese immigrant children's experiences in EC centres requires joint interpretations between the researcher and participants.

\subsection{Outline of the thesis}

The thesis is in nine chapters, each of which is briefly described here to provide an overall context.

Chapter 1 provides background information about the study, the rationale for the selection of the research focus and the research questions.

Chapter 2 sets out the key theoretical concepts underpinning the research topic. Learning and development are discussed from the perspectives of sociocultural theories and their relevance is explained for the ways in which the experiences of the children unfold within this study. The notions of multiculturalism and cultural diversity are also built into this chapter to shape the research focus.

Chapter 3 reviews the literature associated with the learning experiences of Chinese immigrant children in their families and in EC settings. It describes studies exploring the impact of Chinese culture and the culture of immigration countries on child-rearing, parenting styles, and practices of Chinese immigrants. Literature on second language acquisition, social behaviours and learning behaviours of Chinese immigrant children in EC settings is reviewed too. The chapter concludes with a description of some teaching approaches reported as traditionally used with Chinese immigrant children.

Chapter 4 describes the methodological approach of this study, including its conceptual framework, its position of inquiry, and specific research strategies. It justifies the research design, illustrates the process of conducting the fieldwork phase of the study, as well as describes the manner in which the data are analyzed. My own reflection on the research experiences is also discussed. 
Chapter 5 contextualises the research by introducing the study children and providing the perspectives of the children, their parents, and their EC teachers on the learning and development of the children both in their families, and in the EC centres. It also explores the relationships between Chinese immigrant parents and New Zealand EC teachers. This chapter emphasises that learning experiences of the Chinese immigrant children occurred in a sociocultural context. The organization of beliefs and practices of the children, their parents and teachers forms this context.

Chapters 6 and 7 focus on the learning experiences of the study children in their EC centres. The children's experiences with the languages used in the centres and their relationships with peers are described in each of these two chapters.

Chapter 8 brings together the main findings of the research, and offers an overall answer to the two central questions of this research. This chapter provides conceptual analyses of the learning experiences of the children in New Zealand EC centres. These analyses then lead to considerations for educational implications for working with Chinese immigrant children in these centres.

Chapter 9 concludes the study with a synthesis of the arguments of this research, highlighting its contributions, and providing thoughts on further research. Limitations of the study are also discussed.

\subsection{Chapter summary}

The undertaking of this study followed my personal interests which were reinforced by contextual needs in New Zealand EC services, and a lack of a substantial body of scholarship dealing with the chosen issue in existing literature accounts. This chapter has introduced a brief overview of the research in order to set the scene for the rest of the work.

The next chapter describes the theoretical ideas underpinning this study, including the sociocultural ideas proposed by Lev Vygotsky (1978), Etienne Wenger (1998) and Barbara Rogoff (2003) and the relevant concepts of these theories. A number of other concepts related to diverse cultural relations are also discussed. 


\section{Chapter 2: Theoretical underpinnings}

\subsection{Introduction}

This chapter outlines the underpinning theoretical concepts of this research, which primarily derive from sociocultural perspectives. Central to this theoretical choice is the view that an understanding of Chinese immigrant children's learning experiences can be achieved by investigating how the children live in their social and cultural contexts at a particular point in time (Cole, 2003; Rogoff, Mosier, Mistry \& Göncü, 1993). The social and cultural context is interpreted here as a child's living environment including their family and the immediate situational events (García, Lamberty, Jenkins, McAdoo, Crnic, Wasik \& Vazquez, 1996). In this research, the children's social and cultural contexts consist of the cultural community of the EC centres and the Chinese immigrant children's families. Underlying this investigation is an attempt to see how the cultures in these two communities simultaneously or interchangeably influence Chinese immigrant children and how these influences manifest within their learning experiences. The central focus of the study is culture and the emphasis that sociocultural theories place on culture as an integral part of human development influence this study. This theoretical choice can also be attributed to the recognition of the appropriateness of sociocultural positions for understanding and explaining learning experiences of children (Brennan, 2005; Fleer \& Robbins, 2004; Hedges, 2007; Robbins, 2005). Sociocultural theories, according to Fleer (2002), are not only increasingly underpinning early childhood educational research and practice, but also "re-building the theoretical foundations of early childhood education" (p.105).

The theoretical framework of this research draws on a range of perspectives, including (i) the sociocultural position initially framed by Lev Vygotsky (1987) and relevant concepts to this position, to explore learning and development as a sociocultural experience; (ii) the notion of cultural participation and transformation discussed by Barbara Rogoff, and her consideration of cultural variations; (iii) the concepts of multiculturalism and cultural diversity to provide a way to articulate relations between different cultures, and (iv) the perspective of learning communities to consider the shared nature of learning as applied in the relationship between teachers and parents, and in children's creation and performance of identities in a networked and social 
learning approach. These perspectives situate the discussions of this thesis in terms of broader discourses in cultural, social, cross-cultural, multicultural and intercultural levels of learning and development.

\subsection{Sociocultural origins of learning and development}

Lev Vygotsky, the founder of the sociocultural perspective, makes an important point about the interdependent relationship between learning and development. He argues that "properly organised learning results in mental development and sets in motion a variety of developmental processes that would be impossible apart from learning" (1978, p. 90). Vygotsky describes the situation where a child interacts with people in his/her cultural group, or in cooperation with peers, to be a properly organised learning context.

Thus, learning and development are socially and culturally constructed. Vygotsky (1981a) believes that a child's learning and development appears on two planes: "First it appears on the social plane, and then on the psychological plane. First it appears between people as an interpsychological category, and then within the child as an intrapsychological category" (p. 163). Wertsch (1985) takes the view that the key to sociocultural theories is the connections between human beings and their sociocultural contexts.

\subsubsection{Culture}

Culture is the most prominent aspect of the sociocultural perspective. Within it, culture is discussed as incorporating three key components: cultural tools, mediation and social relationships (Cole \& Gajdamaschko, 2007). The development of culturally mediated beliefs and practices via the use of cultural tools and social relationships characterises the process of learning and development.

The sociocultural perspective that highlights the influence of cultural tools, cultural mediation, and social relationships on learning and development is consistent with the focus of the current research on Chinese immigrant children's use of cultural tools and their social relationships. In addition, because sociocultural scholars from Vygotsky to Rogoff have established the unity between learning and development, in this research 
Chinese immigrant children's learning experiences are considered to include both their learning and development.

As already stated, the learning experiences of Chinese immigrant children in New Zealand EC centres should be conceptualised within both their family culture ${ }^{1}$ and the culture of the EC centres because "for the child, everyday concepts are connected to family and community life" (Cole \& Gajdamaschko, 2007, p.204). Given the importance of social relationships for children's learning and development, and the cultural underpinnings of those social relationships, this study investigated the Chinese immigrant children's learning experiences in the context of the relevant cultural experiences of Chinese immigrant parents, New Zealand EC teachers and also the peers of Chinese immigrant children.

\subsubsection{Cultural tools}

Human functioning depends on the cultural tools that are organised in society (Wertsch \& Tulviste, 1996). These include material and symbolic tools (Daniels, 2005; Newman \& Holzman, 1993). Material tools facilitate "the process of natural adaptation by determining the form of labour operations" (Vygotsky, 1981b, p.137), while the symbolic tools direct human minds and behaviours. In sociocultural terms, language, signs and symbols are important symbolic tools (Woolfolk, 2004).

An essential function of cultural tools, particularly the symbolic tools is to express cultural meanings. Of particular importance in relation to people's use of symbolic tools, therefore, is for them to encode the cultural meanings embedded in these tools (Vygotsky, 1981a). Marx William Wartofsky (1979) endorsed a similar view to that of Vygotsky's. Wartofsky emphasised the importance of cultural context in understanding knowledge construction and described cultural modes of representation as utilising cultural tools. According to Wartofsky, cultural modes of representation, namely a distinctively cultural form of tool application, should be explored in order to understand the ways in which individuals retain and manifest cultural meanings within the same

Family culture means the children's culture in their families. This thesis uses this term but not the 'home culture' because home culture commonly refers to one's original cultural heritage and to immigrants, the culture of their original countries. The specific culture that is discussed in relation to the children in this study is the culture in their families. 
cultural group. As such, members of the same cultural groups are "making sense of the world, in broadly similar ways" (Stuart Hall, 2003, p.2).

Nevertheless, it is not the case that individuals within the same cultural communities ascribe similar meanings to their cultures. Children's relationships with their learning environments determine the ways in which they interpret the meanings of their cultures. Vygotsky argued that "any event or situation in a child's environment will have a different effect on him depending on how far the child understands its sense and meaning" (1994, p.343). A key contributor to the learner-environment relationship is the characteristic of learners in their process of development. For this reason, the same cultural environment influences different learners in dissimilar ways.

Two implications of these views in research are: that people's behaviours associated with their use of cultural tools to make meanings of a culture are matters for investigation if one intends to understand the influence of a culture on its members; and that culture has different influences on people in the same community.

In relation to this research, cultural tools provide an explanation of how culture, as an abstract notion, visibly operates on and among human beings. Within this notion, an investigation of children's learning experiences must focus on children's operation of cultural tools, and on the ways in which this operation influences their behaviours. Given that individuals vary in the extent to which they relate to a culture, the influence of Chinese culture on the children in this study needs to be viewed in an open sense, in recognition of the different environments of the EC centres. These foci provide an understanding of children's learning as being purposeful, supported, evolving, and contextualised, and lead to a reading of them creating, sorting out, and utilizing cultural means of support.

\subsection{Language}

Vygotsky has given significant thought to language and viewed it as the most significant symbolic tool. Wertsch and Tulviste (2005) note that Vygotsky defined culture mainly in terms of symbolic systems, and the symbolic system that appeared most in his theories was language. In practical research, this means that researchers seek evidence about children's engagement with language. Vygotsky studied language in two 
ways. The first was to make links between language and thoughts, and the second was to consider language as a device that served specific social practices.

For Vygotsky, language helps complete thoughts. Mooney (2000) said "Vygotsky believes that language presents the shared experience necessary for building cognitive development" (p. 89). In the early childhood years, language is a powerful tool that supports children to make sense of their world. With reference to Wartofsky's concept of cultural representation discussed above, language is a cultural mode of representation, because it retains and manifests cultural meanings.

When considering the links between language and thought, Vygotsky (1978) described examples of children's private speech. He distinguished social speech from private speech, claiming that children processed information and thought about their actions when speaking to themselves. Private speech is located in a social context, functioning to connect thoughts and words with actions. Private speech transforms "the social method of behaviour into a function of individual adaptation" (Vygotsky \& Luria, 1996, p. 168). In this way, Vygotsky also brought to life the nature of language to crystallise cultural meanings (Minick, 2005; Mooney, 2000).

In addition to its role in supporting thinking, language is also perceived by Vygotsky as a tool for social operation. Language assists people to implement their social roles (Minick, Stone \& Forman, 1993), and the use of a language is "a cultural practice with specific rules and tools" (van Oers, 2007, p.301) "in socially appropriate ways" (p.300). Taking inspiration from Vygotsky's approach, John-Steiner and Tatter (1983) wrote:

From birth, the social forms of child-caretaker interactions, the tools used by humans in society to manipulate the environment, the culturally institutionalized patterns of social relations, and language operating together as a socio-semiotic system are used by the child in cooperation with adults to organize behaviour, perception, memory and complex mental processes. For children, the development of language is a development of social existence into individuated persons and into culture. (p.83)

If language is an important tool for social connection, internal thinking and behavioural guidance, then it is a particular area for research on children's learning and development. Specifically, research investigating children's use of language to talk both to themselves and with others should provide significant information about how children locate themselves in a cultural setting. 


\subsubsection{Cultural mediation}

Associated with the application of cultural tools is the notion of mediation. In sociocultural theories, one's contact with the world is a mediated process, and learning is the result of the mediation of actions through tools.

Mediation is a process in which individuals operate with cultural tools, and in doing so negotiate and apply meanings within their cultures. Because of this, "mediation is not restricted to human capacities or manufactured objects; it could be a feature of the landscape representing the connection between people and place" (McDonald, Le, Higgins \& Podmore, 2005, p.115). The fundamental contribution of mediation to human development, therefore, rests on its support of people's attempts to connect themselves with a cultural world (Vygotsky, 1998).

The 'making sense' process is a product of two mediational forces: language and social relationships. According to Daniels (2005) the mediational dimensions of language and social relationships are essential components of their capacity to serve as cultural tools, thereby to help create and ascribe cultural meanings in learning and development. An investigation into children's learning experiences, therefore, should make attempts to see how language and social relationships act as mediators for children's learning and development.

\subsubsection{Activity theory and activity system}

Activity theory is a sociocultural framework that can explain the complexities of tool mediation. Alexei N. Leont'ev developed it as an extension of Vygotsky's idea of mediation (1981). Leont' ev formed a three-level model of activity, through which he conceptualised learning and development as requiring an object-related motive, conscious goals of individuals and the tools at hand. Within activity theory, an activity is a system of human action, whereby individuals employ tools to achieve a particular object of intention. Leont' ev sees activities as being mediated by the cultural community in which they are implemented. This process involves some forms of labour division and the use of particular rules. 
There are four key principles that underpin activity theory. First, learning is dynamic and evolving. Second, learning is widely distributed but shows the close influence of history, social practices, and values of a particular cultural community. Third, learning is associated with the use of the tools available in the community. Fourth, individuals display agency in their learning "but do so in environments that involve others, environments of people-with-tools that both afford and constrain their actions" (Russell, 2002, p.67).

Closely associated with activity theory is the activity system. The activity system is defined as "a theoretical lens" (Russell, 2002, p.67), "a context", or a "functional system of social/cultural interactions that constitutes behaviour and produces that kind of change called learning” (p.68). Engeström (1987) developed a 'web' model to illustrate the interwoven relationships between the essential elements of an activity, for example, subjects, object/motive, mediating tools, rules, community and division of labour. This is an expansion of Vygotsky's mediational model, demonstrating that learning is not as simple as mediation on a learner through objects but involves many affordances and constraints within and beyond the learner's immediate space, context and time. According to Edwards (2005, p.55), Vygotsky's cultural psychology “is often able to tell us about how specific ways of engaging with the object are enabled or discouraged at the level of the activity system"

Engeström (1987) conceptualised the interaction or coordination of activity systems as 'boundary crossing' or 'expansive learning'. Through his notion of 'object transformation', Engeström talks of learning as occurring in a changing combination of interconnected activity systems towards common goals. Boundary crossing suggests a process of creating new activities. Common or complementary tools as 'boundary objects', are used as an initial means of bridging different activity systems (Star \& Griesemer, 1989).

If the activity system is a context as described above, boundary crossing happens through the interconnection of learning contexts. However, there are usually contradictions in the boundary zones because boundaries represent discontinuities of practice between the activity systems. According to Fürstenau (2003, p.91) "often it is not easy to cross boundaries... Obstacles to boundary-crossing can be such phenomena 
as groupthink or fragmentation of viewpoints". Successful boundary crossing therefore requires the communication, discussion and critical reflection of the involved parties (Engeström, Engeström \& Kärkkäinen, 1995).

It is important that activity theory and the related concepts of activity systems and boundary crossing are incorporated into the discussion of this thesis because these concepts can assist in explaining the observable phenomena of the research as learning across settings and over time. This helps understand Chinese immigrant children's learning and development in their EC centres as a transferable process, in which their engagement with cultural tools, roles and community are expanded. Specifically, these theoretical concepts provide a basis to understand how children agentically weave their wider learning experiences in a sociocultural web, in recognition of the affordance and constraints of their contexts. The boundary crossing provides a useful metaphor to describe how children and other participants use objects to help them coordinate learning experiences across different cultural communities.

\subsubsection{Social relationships}

Social relationships are the central point of the sociocultural arguments. It has been claimed that the best way for humans to learn is through interacting with people who are more capable than the learners themselves (Feden \& Vogel, 2003). Social relationships have also become a focus of attention in research, particularly in terms of their role as a cultural mediator for learning. For example, "in early childhood education, the notion of mediated learning is evident in the focus on relationships that mediate learning" (Anning et al., 2004, p.176). This means that if we want to investigate children's learning and development, serious attention should be paid to the relationships that children develop with others, as well as to how these relationships mediate children's learning and development. For this purpose, the following subsections consider the interpersonal constructs that are relevant to the sociocultural perspectives.

\subsubsection{Peer relationship}

William Corsaro, a renowned child ethnographer, paid significant attention to children's peer relationship and conceptualised it as 'peer culture' (1985; 1997; 2005). In Corsaro's (1997, p.95) own words, peer culture is "a stable set of activities or routines, artefacts, values and concerns that children produce and share in interaction with peers". 
From his perspective, children's strong desire to participate with peers in cultural practices contributes to their establishment of peer culture, which then fosters children's social development. Specifically, Corsaro claimed that "children immensely enjoy simply doing things together" (2005, p.158), and "peers and peer culture are central to children's evolving membership in their culture" (1997, p.112).

The importance of peer relationships for children's development is also highlighted in other research. For example, Krapperman (1996) pointed out that due to their "equal standing" (p.44), peers create appropriate socialization contexts. Deegan (1991) found that the voluntary nature of peer relationships allows children to connect in ways that they prefer, thereby enabling them to make attempts to be creative and sociable. Peers help children "meet important basic needs, including the need for belongingness [sense of belonging], companionships, social acceptance and intimacy" (Malcom, JensenCampbell, Rex-Lear \& Waldrip, 2006, p.722).

Peer culture is characterised by close and stable peer relationships (Corsaro, 2005). These relationships are formed on the basis of two dynamics: the social practices learned in families and children's search for emotional bonds or security. As Corsaro explains, "children are introduced to elements of a more general peer culture and to particular local cultures in the family through interaction with older siblings, from television and other media, and even from parents" (p.133). I perceive him to mean here is that although peer relationships are formed by children themselves, they build on children's experiences in families. Because of this, peer interactions can also be considered to provide children with opportunities to put into practice familiar knowledge and experiences. This assumption is supported by Aboud and Mendelson (1996) who note that young children look for familiarity and similarity and tend to form a bond with peers from similar and familiar groups. Feng et al. (2004) also argue that peer interaction resembles family interaction, and that familiarity with their play mates and play materials helps children form a bond with each other.

As mentioned above, Corsaro (2005) has seen a strong link between children's peer culture and their development of social skills. Specifically, children develop the ability to share through relating to peers (Woods, Boyle \& Hubbard, 1999). Corsaro argues that "although children's cultures are composed of a wide range of language and 
behavioural routines, none are perhaps more symbolic of childhood ethos than sharing rituals" (p.145).

Other major themes revolving around children's peer relationships are: the acquisition of thinking skills; the use of verbal communication (Chang, Li, Lei, Liu, Guo, Wang \& Fung, 2005); the ability to control aggression (Johnson, Ironsmith, Snow \& Poteat, 2000; MacDonald, 1996); and "reciprocal positive affective exchanges" (MacDonald, p.66); as well as companionship, intimacy, and emotional support (Howes, 1996). According to Howes, the use of interactive skills underpins the development of close peer relationships. "Peer friends are important socialisers who make substantial and possibly unique contributions to children's social and cognitive development" (Ladd \& Kochenderfer, 1996, p.322). These studies indicate that the commitment and engagement in friendship relationships contribute to children's development and learning of a wide range of skills.

It is not uncommon that some children have trouble developing peer relationships, perhaps because of dissimilar abilities; different cultural backgrounds (Deegan, 1991; Igoa, 1995; Tabors, 1998); children's passive or aggressive characters; and racial attitudes (Aboud, Mendelson \& Purdy, 2003). In addition, in Newcomb and Bagwell's (1996) research on children's friendships, they found that "friendless children's lack of experience in mutual, dyadic friendship relationships is associated with difficulties in initiating play with peers and in achieving positive peer interactions" (p.312). Given that peer relationships are so important for children, and children have strong desires for peer interactions, a related point is that children who do not have friends are at the risk of feeling lonely, distrustful, emotionally insecure, behaving aggressively and of developing social competence slowly (Brown, Odom \& Buysse, 2002; Johnson et al., 2000; Qualter \& Munn, 2005).

Evidently, children's life experiences are very much affected by their relationships with peers but so also are the peer relationships closely related to individual children's learning contexts, cultural orientations, family backgrounds, learning experiences, and individual abilities. The concept of peer relationships, therefore, implies that to understand them, researchers should investigate the individual, contextual and sociocultural realms within the space in which children relate to peers. 


\subsubsection{Togetherness}

Togetherness is "a complex tendency of forming and maintaining a group" (van Oers \& Hännikäinen, 2001, p.102), and "an affective involvement of an individual in a group's activity" (p.104).This definition gives further explanation of interpersonal relationships in terms of the purpose that underpins these relationships and the way in which they are manifested. The relevance of this concept to sociocultural theories is also perceived by Hännikäinen and van Oers (1999) who comment that "in describing children's activity, Vygotsky himself used a terminology that expressed the sharing feature of this activity with others (sovmestnaja dejatel'nost'), so the 'togetherness-dimension' can be viewed as a core element of the Vygotsky's approach" (p.32). They believe that people relate to each other in order to develop togetherness in both perception and practice. At the heart of interpersonal relationships, according to this perspective, lies the desire of people wanting to belong together for shared activities (Deegan, 1991) "in a shared space" (Hännikäinen, 1998, p.23).

The construction of togetherness is grounded in a number of social practices, such as sharing, creating 'we-ness' and connectedness (Hännikäinen \& van Oers, 1999). In the pursuit of togetherness, one would expect to develop certain skills and adopt strategies (Deegan, 1991). Among all, verbal communication is the most essential skill in constructing and maintaining a sense of togetherness for people (Deegan, 1991; De Hann \& Singer, 2001). In van Oers and Hännikäinen's words (2001), “the language people use is a very strong determinant of group formation and confirmation of a group's togetherness" (p.107).

\subsubsection{Sense of belonging}

A sense of belonging indicates a form of human feeling in group situations. Osterman's (2000) study on children's development of a sense of belonging highlighted its importance for them. Osterman concluded that it is only when members of a group experience a sense of belonging that the group has a real existence for them. This means that having a sense of belonging indicates one's success in developing a group identity. Gibson, Bejinez, Hidalgo and Rolón (2004) agree that a sense of belonging is a similar construct to group membership. 
In her review on students' experiences in schools, Osterman (2000) identified the sense of belonging to be a very strong desire of students, and it was also students' most basic need. She noted that a sense of belonging allowed students to "securely connect with others in the environment and to experience oneself as worthy of love and respect" (p.325). Because of this, the need to belong can become "a pervasive drive to form and maintain at least a minimum quantity of lasting, positive, and significant relationships" (Baumeister \& Leary, 1995, p.497). Beaumeister and Leary further explained that when people considered themselves to belong to a group, they demonstrated increasing abilities to cooperate with others. For this reason, a sense of belonging makes a positive contribution to one's learning and development.

Te Whäriki specifically identifies a sense of belonging as a learning goal for all children in EC services. Similar to the viewpoints above, the establishment of this goal is also derived from a reason that "the feeling of belonging, in the widest sense, contributes to [children's] inner well-being, security and identity" (Ministry of Education, 1996, p.54).

Given that a sense of belonging is a strong desire of children in developing themselves in group situations, and it is a government requirement for New Zealand EC settings, it is reasonable to expect that paying attention to the establishment and maintenance of a sense of belonging of the Chinese immigrant children is a vital component of understanding this research topic.

\subsubsection{The zone of proximal development}

The zone of proximal development (ZPD) is envisaged by Vygotsky to be an indicator of a child's potential to learn. "The ZPD is the distance between the actual developmental level as determined by independent problem solving and the level of potential development as determined through problem solving under adult guidance or in collaboration with more capable peers" (Vygotsky, 1978, p.86). Vygotsky saw learning as a reflection of "the relationship between 'matured' and 'maturing' processes" (Newman \& Holzman, 1993, p.56). For him, a child can achieve a higher level of functioning in collaboration with others than alone. According to Hatano (1993), the ZPD is an explicit interpretation of Vygotsky's emphasis on the social origin of human development, and is an image that visualises the integration and coherence of the inner ability of a person and their social support. For this reason, the ZPD presents 
itself as a concept with great teaching implications, because it directs teachers to provide guidance to students within students' zones of proximal development. This means that teacher instructions should be made within an existing ZPD and entail the creation of a further ZPD to trigger the process of children's inner development (Hedegaard, 1990).

\subsection{Learning as guided transformation of participation in families and in cultural communities}

Learning and development can be viewed as the result of the "transformation of participation" (Rogoff, Matusov \& White, 1996, p.388). Through developing this perspective, Barbara Rogoff, another prominent figure of the sociocultural school of thoughts, argues that when individuals participate in cultural activities, they prepare for involvement with future related activities.

Rogoff's consideration of 'culture' is rather straightforward. It is where we see her most clearly coining 'cultural community', a concept involving "people trying to accomplish some things together, with some stability of involvement and attention to the ways they relate to each other" (2003, p.80). In this statement, Rogoff indicates culture as a somewhat integrated set of values, practices, activities, and goals of the people in a community.

Rogoff emphasises repetition as a useful approach to adopt values and practices of a cultural community, describing how "tacit, routine expectations of everyday life are likely to be among the most powerful cultural experiences" (2003, p.491). She considers both direct interactions with other cultural members and indirect interpersonal arrangements of cultural activities as important dimensions of cultural experiences.

For Rogoff, families are the most fundamental cultural community. Many of her studies of children involved families as the site of research and family members as key participants (Chavajay \& Rogoff, 1999; Rogoff \& Angelillo, 2002; Rogoff, Moore, Najafi, Dexter, Correa-Chavez \& Solis, 2007). For example, in a research project on children's participation in cultural activities, Rogoff and her co-researchers noted: 
Our observations were videotaped in a home visit involving child-rearing questions and the opportunity to observe the children and their families in everyday problem solving: exploring novel objects (which we supplied), playing social games, handling the feeding implements of their community, being dressed, and treating other people appropriately. (Rogoff, Mosier, Mistry \& Göncü, 1998, p.226)

Here Rogoff and co-researchers drew attention to families and family members as having primary importance in children's learning and development. An implication for researchers is for them to investigate how children live in their family in order to understand how children relate to a culture they have been born into.

Rogoff (2003) identified three sets of interrelated sociocultural elements. Firstly, participation and learning to participate occur simultaneously. Secondly, participation and contributions can happen together. Thirdly, the process of human development constitutes, and is constituted by, social experiences. In her portrait of human development, Rogoff (p.254) wrote: “A person develops through participation in an activity, changing to be involved in the situation at hand in ways that contribute both to the ongoing event and to the person's preparation for involvement in other similar events".

In sum, the analysis of learning and development assumes that a developing person and his/her social partners and cultural experiences are inseparable. Thus an investigation into the person's transformation of participation should consider person-person-culture interactions. More specifically, Rogoff uses the notion of 'guided participation' to describe the dynamic of these interactions.

Guided participation is a process in which children and other people participate in cultural systems of practice, by learning together and extending skills, values and knowledge of the cultural community (Rogoff et al., 1998). Children's learning and development occur in the form of gradually advancing cultural understanding, and is the result of increasing repertories of participation in cultural practices. Guided participation is premised on the belief that children are powerful and active learners, furthermore it considers the efforts of their social partners and the context of their cultural communities as integral to the process. Thus guided participation is "an attempt to keep individual, interpersonal and cultural processes simultaneously in focus, 
representing inseparable aspects of whole events in which children and communities develop" (Rogoff, Mistry, Göncü, Mosier, Chavajay \& Heath, 1993, p.5).

It can be seen from Rogoff's explanation of guided participation as noted above that she takes the view that individual learning and cultural development function together. This view is different from that of 'internalization' coined by Vygotsky. 'Internalization' highlights the inner ability of individuals to transfer activity of external knowledge into internal dimensions, thus, developing a boundary between the 'interpersonal' and 'intrapersonal' process of learning (Daniels, 2001, p.7). The basic concept behind Rogoff's 'guided participation' is one in which there is a clear belief that the innate processes of learning are constituted by interpersonal and community processes in sociocultural activities. In Rogoff's own words, "the individual is viewed as participating in and contributing to sociocultural activity [and] there is no impermeable boundary between individual and environment" (2003, p.269).

Of particular importance in guided participation is the transmission of cultural information from skilled cultural members to new learners through shared endeavours. Embedded in this process is mutual structuring of each other's perspectives, where participants seek common understandings and opportunities for learning to take place. It emphasises children as equals in their involvement with others in a diversity of relationships and activities. Unlike Vygotsky, who considers interactive activities as the way to enable human cooperation, Rogoff (2003) perceives learning with others to include a wide range of experiences such as through observation as onlookers. She says that learning can happen not only in proximal and explicit ways but "also the side-byside or distal arrangements of activity without co-presence" (p.284).

\subsubsection{Dealing with cultural variations}

For Rogoff (2003), there is no description of culture that is universal. As a result of a wide range of research, she describes how variations occur across cultural communities in the form of people's expectations and practices. Therefore, there is a significant implication particularly for those children in New Zealand "whose community ways differ from the ways of Western schooling" (p.256) because these children may have difficulties finding connections between the two (or more) contexts they are experiencing. 
Within the variations in cultural practices, Rogoff and co-researchers (Rogoff et al., 2007) highlight the importance of children's creation of "hybrid forms" of practices (p.509), to find ways to participate in cultural communities different from their own. They claim that the cultural practices established by the children are critical to their participation in unknown situations, and that there might then be an adjustment or redevelopment of their familiar repertoires to produce "their own repertoire of practice" (p.491) to cope with the new situational demands. According to Rogoff et al. "faced with home/school discontinuities, children (and parents) often adjust their ways of participating; they may adopt school ways, or they may develop hybrid forms" (p.509).

The dimensions of hybrid forms of practice and repertoire of practice highlighted in this section are significant learning components in settings where different cultures exist. The important point here is that within their family or cultural community, children develop fundamental cultural skills or strategies of seeing and doing things which assist their participation in other cultural communities. These concepts address how children can make contributions to their own learning processes by finding out "which strategies are helpful in what circumstances" (Rogoff et al., 2007, p.510).

In relation to current research, these ideas from Rogoff and her colleagues indicate the importance of the processes in which Chinese immigrant children become members of another cultural community. They do so through the involvement of their family knowledge and experiences in the events of the new cultural community. The view that learning and development are a guided transformation of participation suggests that this investigation should be conducted in a multifaceted manner, that focuses not only on the participating children, but also on the people in different contexts of their lives. It is important too to recognise that children's learning and development are dynamic and transformative, being influenced by their social partners and learning contexts.

\subsection{Concepts relating to multiculturalism and diverse cultural relations}

This chapter includes an exploration of the concepts related to diverse cultural relations. With reference to the above-mentioned perspectives about culture, cultural mediation, cultural community, cultural practice, activity system, boundary crossing and cultural variation, it is reasonable to expect that Chinese immigrant children's learning 
experiences relate both to their families and their EC centres. The children's learning experiences in New Zealand EC centres, therefore, would reflect the relations of beliefs and practices between two different cultures. For this reason, a theoretical framework that captures the essential aspects of diverse cultural relationships is a crucial component of a comprehensive understanding of the topic for the present research. Therefore, ideas and notions pertinent to the present research such as multiculturalism and cultural diversity are now explored.

\subsubsection{Multiculturalism}

The 'multicultural perspective' entered scholarly discussion in the 1960s in response to the trend of immigration to Western countries. The initial aim of this perspective was to listen to the voices of ethnic minority people (Banks, 2006; May, 1999; Ryan, 1999). Over the past forty years, various attempts have been made to address the issues for people from minority and majority cultures living together (Brisk, 1998; May). Despite the fact that multiculturalism is still not an obvious practice (Larkin \& Sleeter, 1995; May, 2004), people's acknowledgement of the limitations of monoculturalism has increased as a result of these attempts (Todd, 1991).

It has been argued that the main challenges regarding implementing multiculturalism are those of power, identity and difference (Larkin \& Sleeter, 1995; May, 1999; Modood \& May, 2001; Ritchie, 2003). According to May, the idea of multiculturalism finds resistance because people of the predominant cultural group are afraid that bringing other cultures into their own cultural group threatens the legitimacy of their current power, identity and social systems. May names this resistance as "cultural essentialism" (p.12), in that the fluid feature of life contexts is ignored and a single culture becomes "necessary" (p.32), "fundamental [and] immutable" (p.34).

In discussing the cultural relationships in the New Zealand EC sector, Ritchie (2003, p.3) points out "the dominant Pākehā mainstream culture" as the culture of power that is embedded in the teaching programmes of EC services. She discovers the reason for this is that "Pākehā educators, as representative of the dominant culture, are able to exercise power [in their teaching practice] because their discourses have become institutionalised as normal, right, and desirable, thus privileging these people and silencing and marginalizing alternative discourses". 
For May (1999), the implementation of multicultural practices is a matter of balancing the power of the dominant monoculture and the cultures of those who differ from it. May, Modood and Squires (2004) point out that multicultural practices should interconnect with cultural differences, and accept the beliefs and practices of the minorities as unique and dynamic components of a national identity. In relation to education, May suggests that schools adopt an accommodating approach to nondominant forms of knowledge, "allied to a critique of wider power relationship", and undergo "an ongoing and recursive process of cultural construction and reconstruction (p.34).

\subsubsection{Cultural diversity and the difficulties in implementing cultural diversity}

Michael Cole is another scholar working from a sociocultural perspective. His theoretical position, similar to that of Rogoff's cultural variation, is that cultural diversity is an important factor when considering human experiences. Cole's (1988; 1998; 2005) view is based on a strong belief that life experiences are extremely contextspecific, and therefore should be understood in line with the contexts in which they occur. In his opinion, the everyday encounters of people should be viewed against a multidisciplinary framework; variations and differences embedded within and across cultures need to be recognised.

In the education sector, noticeable interest in cultural diversity emerged in the 1970s when educators began to realise that "an 'ever-changing and diversifying process' exists because of our relationships with other people, other discourses, and other positioning" (Rhedding-Jones, 2005, p.142). However, responses to cultural diversity encountered difficulties and resistance. Until very recently, cultural diversity is still an ambiguous term associated with multiple interpretations and superficial applications (de Melendez \& Beck, 2007).

A major underlying issue for supporting cultural diversity is people's conceptions of culture. Williams (1958, as cited in Ryan, 1999) contends that, "cculture' is one of the three most complicated words in the English language" (p. 55). Banks and Banks (1993) claim too, that a significant issue in relation to multicultural education is that teachers, policy makers and the general public oversimplify the concept of 'culture'. 
Generalizing culture adds a further complexity to the work on cultural diversity or multiculturalism. Such examples have been documented by Bennett (2006), who has discussed cases in her personal experiences that "multicultural education focused primarily on ethnic groups within one society" (p.11). In much the same way, in relation to ECE, Siraj-Blatchford (2001) points out that there is a generalization of cultural groups which results in an over-representation of cultural diversities. She means here that diversity within a culture has been overlooked in the ECE sector. Rhedding-Jones (2005) also gives evidence that the term diversity is commonly defined in a singular way. Similarly to Siraj-Blatchford, Rhedding-Jones contends that it is mistaken to see culture in terms of a single group of people.

The emphasis on equity and harmonious relationships in society could serve as another challenge to the incorporation of cultural differences. If the emphasis on social equity is underpinned by the idea that cultural convergence promotes coherence and harmony (Dijkstra, Geuijen \& de Ruijter, 2001), and it is possible to avoid difference because the beliefs and practices of mainstream cultures are neutral (Banks, 2001), then diversities would not be addressed because they take a different stance from that (Brewer, 2007). Moreover, since "a problem with ... understanding of diversity is that they [diversities] appear to require an 'add-on' multiculturalism that 'celebrates' exotic otherness" (Laubscher \& Powell, 2003, p.221), a focus on diversities has a danger of labelling differences as 'others' that need to be excluded from the mainstream, which thus bring challenges to the emphasis on social equity and harmony.

The importance of cultural diversity and the difficulties in addressing it in multicultural societies mean that cultural diversity is an interesting conceptual structure for the focus of this research. This enables the research to move beyond an interpretation of the learning experiences of Chinese immigrant children to attend to the wider sociocultural contexts which the children experienced. By including the notion of diverse cultural relations into the sociocultural approach, it is possible to establish an intercultural construct in the sociocultural approach in which the learning contexts of the Chinese children in New Zealand EC centres may be viewed through a broader lens. This construct can guide the present research to examine beliefs and practices in the two different cultural communities, and more specifically how their differences influenced the experiences of the Chinese immigrant children in New Zealand EC centres. 


\subsection{Learning communities}

The concept of 'learning communities' focuses on relationships. This concept has gained popularity in contemporary educational fields (Fleer, 2003; Hall, 2003; Hughes, 2003) and has been used variously within literature, in parallel with, for example, ‘communities of practice' (Lave \& Wenger, 1991; Wenger, 1998).

Generally speaking, the notion of learning communities requires consideration of the relationships between people when they learn together. According to Hall (2003), the concept of a learning community emphasises the relational nature of learning. Underlying this concept is an expectation that every member in a group contributes to the group outcome as a result of their knowledge about the group system and function (Marsick \& Kasl, 1997). Because of this, aiming for a learning community is viewed as a practice that balances individuals and groups. For Hall, it involves negotiation and the establishment of shared ways of working together. Daniels (2001) finds links between the notions of learning community and ZPD, and describes "a learning community as a context with which multiple zones of proximal development are in place at the same time (p.119). He alerts us to the fact that a learning community is a dynamic mediator of sociocultural practice.

Hughes (2003) discusses the term 'learning communities' in the context of relationships between teachers and parents in ECE programmes. He, alongside others (Billman, Geddes \& Hedges, 2005), believes that the term resembles teacher-parent partnerships or home-school linkages that have been highly recommended for teaching practices. Within Hughes' discussions, the formation of learning communities between parents and teachers "promotes democratic citizenship by inviting parents and others to form policies, manage resources and evaluate service" (p.269). The establishment of learning communities focuses on the members' mutual engagement in a joint task through sharing experiences and expertise (McNaughton, 2002). Of particular importance for this practice is the establishment of a group identity that evolves around the meaningful practice of all members.

In New Zealand, the establishment of learning communities is also an aspiration for ECE practice. For example, in her discussion of assessment of children in EC settings, Carr (2001, p.157) noted that "practitioners got to know the children, planned for their 
learning, and established a learning community with shared values". A focus on learning communities is also evident in Kei tua o te pae, Assessment for Learning (Ministry of Education, 2004). "The exemplars indicate that there is a learning community that is involved in both curriculum and assessment. The learning community includes children, families, whānau, teachers, and others" (Ministry of Education, p.3).

Wenger's (1998) concept of 'communities of practice' shows a similar focus to 'learning communities', describing groups that interact to achieve a shared purpose. However, he expands that idea by also giving thought to the ways in which individuals develop and learn as a result of them engaging with their communities. Learning entails changing abilities, ascribing meanings of experiences, and developing a sense of identity in sociocultural practices (Wenger, 1998).

In some sense 'communities of practice' integrates Rogoff's idea of participation and Wenger's notion of 'boundary crossing', in that it considers development as an evolving participation in a community and a crossing of boundaries. However, what is more noticeable about the notion of a community of practice is its emphasis on the identity of individuals in their communities and the meanings they construct, negotiate and consolidate across time and space. The experiences of people in their communities of practice include forming identities, undertaking social practices and developing their “"own' meanings" of their experiences (Wenger, 1998, p.15). For Wenger, community, identity, membership and meaning are mutually constituted in learning.

\subsection{Chapter summary}

This chapter has discussed some key ideas that underpin this research. They act as theoretical and conceptual supports to help me understand the issues and dynamics raised in this research.

I gained significant inspiration from sociocultural ideas including the beliefs of Vygotsky, Rogoff and others in the power of culture, cultural tools and cultural mediation in human learning. These highlight the importance of language, interpersonal relationships, and the ZPD in explaining learning and development. These ideas provided conceptual tools with which I was able to contemplate the learning 
experiences of Chinese immigrant children as a process mediated by their social experiences, through the use of languages.

Rogoff's approach of transformation of cultural participation in guided practice has implications for my study which looks at children's evolving participation in cultural practices within EC settings in New Zealand. It suggests the need to study how children and their teachers, parents and peers participated in shared learning experiences and how, and to what extent, these experiences guided the children's learning and development. Insights arising from Rogoff's ideas about culture, cultural community, and variations in cultural practices, as well as children's own creation of a hybrid response to cultural variations, are similarly helpful to this study.

In sociocultural theories, culture is an integrated set of variables, including the practices, beliefs, and expectations of the people in a community, who relate to each other through cultural tools, directly or otherwise. This idea becomes a fundamental concept in this research as it indicates what constitutes culture in the children's families and their EC centres.

Concepts from activity theory, of activity systems and boundary crossings have constructed views of expansive and distributed learning (Russell, 2002; Wenger, 1998). Within these views, learning involves an engagement with cultural affordances and constraints, and a crossing of boundaries through the use of common boundary objects. These conceptual ideas suggested that I needed to recognise that my understanding of Chinese immigrant children should draw upon the relationships between the children's two activity systems in their lives, namely the activity system in their families and that operating in the EC centres. Although the primary focus of this study was on the children's learning experiences in their EC centres, the ways in which they crossed the boundary between these two zones were clearly relevant to my study.

The perspectives of multiculturalism, cultural diversity and the notion of learning communities provide further lenses through which I can refocus my understanding of Chinese immigrant children's immediate experiences and place them in a wider sociocultural context. These perspectives provide an essential theoretical foundation for this study. They also enable insights to explain how Chinese immigrant children and 
their families, as people from minority cultural communities, experienced their lives in the dominant Pākehā mainstream cultural community of the EC centres (Ritchie, 2003).

Engaging in communities of practice means the evolution of full participation in sociocultural practices. In this process, children develop a sense of identity, and construct and discover meanings of their experiences. The analysis of this perspective suggested that a significant undertaking of my research should be an attempt to capture the children's sense of community membership and their interpretation of their experiences.

The following chapter reviews existing literature on the learning experiences of Chinese immigrant children. It is structured around three key components: learning experiences of Chinese children in Chinese immigrant families; Chinese immigrant children in nonChinese speaking EC settings; and the role of teachers who work with Chinese immigrant children. 


\title{
Chapter 3: Literature review on the learning experiences of Chinese immigrant children
}

\begin{abstract}
My daughter arrived in New Zealand when she was five and a half years old. She started school as soon as she came to the country. Regardless of her experiences at a Kiwi primary school, once my daughter was back home, she was exposed to many Chinese things: Chinese food, Chinese friends, Chinese television programmes, Chinese festivals, Chinese language, Chinese pictures, Chinese stories and Chinese language classes. When she was naughty, my husband and I still used the same ways we used in China to tell her off or discipline her by depriving her of her toys. Upon reflection, I wonder how my daughter experienced a non-Chinese learning environment as a result of her learning experiences in a Chinese immigrant family. (Journal Reflections, August, 2004)
\end{abstract}

\subsection{Introduction}

My daughter's experience led me to realise that Chinese culture could be maintained in Chinese immigrant families and that the family experiences of these children might influence their learning experiences in non-Chinese learning settings. The work of Rogoff (2003) reporting the different learning behaviours of children across cultures, due to 'guided participation' in communities, is a good reference point for the way in which this chapter is presented. Moreover, given that sociocultural research with children focuses on their learning contexts in both their families and EC services (Adair \& Tobin, 2008; Hedges, 2007; Pacini-Ketchabaw \& Schecter, 2002), an understanding of Chinese immigrant children's learning experiences in non-Chinese cultural communities would be more profound if a knowledge base about their families is established as a backdrop.

This chapter begins with information about rearing children in Chinese immigrant families, followed by a review of literature on Chinese immigrant children's learning experiences in non-Chinese EC settings.

In thinking about family or culture as it relates to learning and development, I have found it useful to begin with a statement that "immigrant individuals vary in the extent to which they strive to maintain traditions and values from their culture of origin, as well as the extent to which they adopt the features of the new culture" (Costigan \& Su, 2004, p.518). This acknowledges that there is great variability among Chinese 
immigrant children's learning experiences in their families and the wider cultural community, and therefore the material reviewed in this chapter provides only general ideas on the topic.

\section{1 ${ }^{2}$ Children in Chinese immigrant families}

\subsubsection{Traditional Chinese cultural orientation to childrearing}

Recent studies show that Chinese immigrant children develop in line with many traditional Chinese beliefs about child development, even when living in a place not of their origin (Chen, 2001; Ebbeck \& Gokhale, 2004; Huntsinger et al., 2000; Lin \& Fu, 1990; Zhang, Kohnstamm, Slotboom, Elphick \& Cheung, 2002). Chen concluded in his comparative study of Chinese parents, Chinese American parents and American parents that in terms of raising and educating children, "no significant differences were found between the Chinese and Chinese-American groups" (p.310). Chan's (2004) research on recent Chinese immigrants in Australia indicates that in order to establish a sense of security, Chinese immigrants try to be more Chinese than the Chinese in China.

The ideas of Chinese philosophers, including Confucius, Mencius and Hsun Tzu have influenced how children are brought up (Chan, 2004). While these philosophers differ in their views of human nature, they all believe that regardless of one's genetic heritage, all humans can be cultivated for a good end. A core idea in Chinese philosophy is that nurturing can make a difference. Among all the philosophers, Confucius is considered by many as the most influential in guiding people's view of human development (Chan; Chen, 2001; Li \& Wang, 2004; Lin \& Fu, 1990; Shek \& Chan, 1999). Confucian thinking has permeated into many, if not all, aspects of childrearing and education in Chinese culture (Chan).

\subsubsection{Self perfection and the development of proper characteristics}

In Confucian terms, learning is aligned with the development of 'the proper characteristics' of self perfection (Li \& Wang, 2004). An ideal person, by Confucian standards, shows self restraint, controls feelings and emotions, maintains harmonious relationships, and pursues knowledge to the best of one's ability with a goal of developing compliant characteristics (Shek \& Chan, 1999). Restrained and compliant

\footnotetext{
${ }^{2}$ Parts of this chapter have been first published in Guo, K (2006). Australian Journal of Early Childhood, 31(2).
} 
characteristics are the precursors of harmonious relationships. The development of these characteristics leads one into a purposeful life, including playing the role of a responsible family member. Among his many ideas, Confucius' conceptualization of 'family' provides important insights for understanding the human characteristics expected in Chinese culture. 'Family' in Confucian thought revolves around two notions: the collectivist tradition, and the ethic of filial piety. The former characterises the nature of interdependence in families, and the latter highlights the importance of obeying and honouring seniors (Huntsinger et al., 2000). This extends to the wider political realm because Chinese people believe that it is important "to regulate the family before one can rule the state" (Shek, 1996, p.86). Confucius emphasises that "children achieve for their family" (Huntsinger et al., p. 8).

To develop proper characteristics, "self-cultivation" is the most favoured approach (Shek \& Chan, 1999, p.293). It is achieved by learning shame and self blame. Fung (1999, p.182) confirms that "Chinese culture is rather a 'shame-socialised culture' in which individuals are strongly socialised to be aware of what others think of them, and are encouraged to act so as to maximise the positive esteem they are granted from others, while trying to avoid incurring their disapproval".

Closely related to shame or self-blame is the emphasis on loss of face. According to $\mathrm{Hu}$ (1944), the concept of 'face' is both interesting and powerful in Chinese culture because it is not directly understood as the physical face but mien $t z u$, a recognition ego, meaning the respect from other people. The loss of face "makes it impossible for him [a person] to function properly within the community" (p.45). Once face is lost, a person becomes ashamed as they lose social respect. Therefore, keeping face is an important aspect of one's strategies in life. For this reason, in Chinese culture, learning and development are devoted to one's desire to maintain a respectable social image in order to prevent a loss of face (Chang \& Holt, 1994). Mien tzu, thus, reveals that Chinese people are deeply concerned about their social values and social judgments (Chang \& Holt).

\subsubsection{Working hard for academic success}

"Confucian thought strongly emphasises individual intellectual development, skill acquisition and love for learning" (Li \& Wang, 2004, p.416), and academic success is 
the eventual goal. For many Chinese, this provides the fuel for upward social mobility and reflects the achievement of a person. Along with academic achievement, Chinese culture stresses the importance of working hard (Li \& Wang; Shek \& Chan, 1999). In Chinese culture, the most important dimension of seeking knowledge is the concept of "haoxuexin" (Li, 2004, p.126), meaning having a heart of learning. Chinese consider that hereditary factors are not as important as social environment and "they believe that one can go beyond what nature has given" (Li \& Wang, p. 419). A bright person will not achieve much if he or she does not work hard, while a slow person can achieve more if that person continues to strive. A similar view is even expressed by Chinese preschoolers, who were reported by $\mathrm{Li}$ and Wang to have claimed that "people who make the effort are smart, and smart people work hard" (p.417). Socialization for academic achievement in childhood involves building academic skills and cultivating a hard working spirit so that a child can succeed in the school environment (Chao, 1995). According to Li, this spirit does not simply reflect one's determination for academic achievement but has social and moral implications because "if a person is perceived as refusing to learn, he or she may be regarded as socially irresponsible (for parents and family), and worse yet, immoral (not wanting to strive to be good)" (p. 126).

\subsubsection{Immigration life of Chinese immigrant parents and children}

Research indicates that immigration results in an alteration of immigrants' traditional beliefs and values. For example, Goldman (1993) used the term 'cultural lag' to describe the difference between resistance and changes that inevitably occur in cultural values. Margo's (1993) more in-depth analysis differentiated between “"pragmatic values', which are likely to change during social and cultural transitions and central 'core values' which resist such changes" (as cited in Roer-Strier \& Rosenthal, 2001, p.220). These pragmatic and core values are individual and subject to immigrants' own interpretations and perceptions of the host country and the original culture (Roer-Strier

$\&$ Rosenthal). Having researched the adjustment process of immigrants, Sharlin and Moin (2001) claimed that the beliefs and attitudes of immigrants towards life in the host culture were formed through comparison between their life before and after immigration. Immigration has been portrayed as a dynamic and struggling process like "walking a delicate tightrope" (Kibria, 1993, as cited in Foner, 1999, p.257), in which immigrants challenge certain aspects of their traditional beliefs while also trying to retain others to strategically form a satisfactory personal life in a new society. 
A change in traditional Chinese cultural beliefs among Chinese immigrant families is reported in literature. In view of the inevitable exposure to different cultural values and life realities in the new environments, it is not surprising that Chinese immigrant families change their beliefs and practices (Parette, Chuang \& Huer, 2004). Zhang et al. (2002) gathered evidence of Chinese people everywhere possessing some common deeply rooted cultural traits as well as some additional ones nurtured by their respective environments. An example is Lin and Fu's study (1990) compared the childrearing beliefs and practices among Chinese and immigrant Chinese in U.S.A. in which they observed the adaptability of the Chinese immigrants to the conditions of life and social structures of the United States. Similarly, Li's (2001) study of Canadian Chinese immigrants revealed that they were motivated to conform to the Canadian sociocultural context. This is because Chinese immigrants tend to develop community membership in the new society (Parette et al., 2004).

The most identifiable variable that contributes to immigrants' settling in new countries is the extent to which they respond to changes (Jain \& Belsky, 1997; Li, 2001; Sharlin \& Moin, 2001; Souto-Manning, 2007). Amongst these responses, is the influence on the ways that immigrant parents raise children.

\subsubsection{Responding to changes in parents}

Immigration is characterised by "determination and hesitation, expectations and apprehensions, and dreams and worries" (Li, 2001, p.489). When immigrants adapt to a new and culturally unfamiliar environment they face a high level of uncertainty (Sharlin \& Moin, 2001; Souto-Manning, 2007). Schnittker (2002) concluded in his study of the acculturative process of immigrants that immigrants needed to respond to the mismatch between their own practices and those of others in the host country. These practices could include the use of language; implementation of values; roles and norms of family and social interactions; cultural participation; and how parents socialise their children (Foner, 1999; Jambunathan, Burts \& Pierce, 2000; Portes \& MacLeod, 1999). Florsheim (1997) raised the point in an earlier study that immigration brings parents into a perplexing position of not knowing how to apply parenting skills acquired in one context, to facilitate their children's development in a different one. 
Despite the changes, research has shown that most Chinese immigrant parents maintain their parental efficacy (Schnittker, 2002; Souto-Manning, 2007). Underpinning this are the parents' beliefs in the importance of their role as parents and their expectations for their children's success in a new society (Adler, 2001). Regarding this, Chinese immigrant parents appear to be able to adapt their parental styles to the needs of new societies. Studies suggest that Chinese immigrant parents exercise their parental roles in immigration societies in two ways: firstly by utilizing their own accumulated skills and strategies; and secondly by providing their children with opportunities to develop biculturalism (Gorman, 1998; Jose, Huntsinger, Huntsinger \& Liaw, 2000; Li, 2001; Portes \& MacLeod, 1999).

In some countries, the skill-based immigration system generates an influx of welleducated Chinese immigrants. According to Li (2001) in Canada, and Ng (1998) and White, Watts and Trlin (2002) in New Zealand, the majority of recent Chinese immigrants are highly educated urban professionals. "Given their [Chinese immigrants'] educational and professional qualifications, it is not surprising that these immigrant parents wanted their children to secure a good life through education" (Li, p.491). Thus, Chinese immigrant parents support their children to improve their lives through providing them with education and support them to excel in education. As parents' expectations were derived from their life experiences, Chinese immigrant parents' previous experiences significantly influenced their expectations for their children (Li \& Dyson (2001). Successful adaptation of Asian immigrant children, therefore, reflects the education of their parents (Portes \& MacLeod, 1999; Suizzo, 2007).

Li (2001) reported on Chinese parents' beliefs in the effectiveness of traditional values to guide human development. These parents claimed, "children who grow up in Chinese culture are generally motivated to pursue excellence" (p.482), and "the demanding nature of Chinese parenting could ultimately produce positive outcomes" (p.484). In their study of the values and practices of first-generation Chinese in the United States, Jose et al. (2000) noted the endorsement of traditional Chinese values of those people. For example, they observed the Chinese immigrants to exert clear parental control over their children, and these parents were more directive than their American peers. 
The development and maintenance of a sense of original culture is also reflected in parental input into the construction of their children's original ethnic identity. In her study of Asian-American children's ethnic identity formation, Adler (2001) found "these parents socialise their children about race and ethnicity" (p.285). She commented that in comparison with parents of other minority cultures "Asian culture... was passed down in a more explicit way as most of the families celebrated ethnic holidays, especially their New Year, and many sent their children to some form of ethnic school to learn the culture and language" (p.286). This, for Adler, revolved around the parents' desire to model ethnic pride to their children. Given that "children as young as 3 years old are very aware of racial difference and absorb the attitudes of those around them regarding a particular racial or ethnic group" (p.274), it is clear that the purposeful construction of ethnic identity by Asian parents raises their children's awareness of being Asians.

It has been found that Chinese immigrant parents positively maintained their first language with their children and they also supported children to grasp the new language (Gorman, 1998). Li (2006) wrote in his case studies of Chinese Canadian school children that, "all families expected their children to become biliterate" (p.355). Huntsinger et al. (2000) described how Chinese immigrant parents reinforced their language with their children at home and also hired native English-speaking children to read English stories to their children. Chao (1995) illustrated Chinese immigrant parents involving their children in English language lessons after school time to compensate for their language limitations. This means that "English was viewed positively because of its importance as a route to success in English society" (Woods et al., 1999, p.192). After studying Asian-American children, Adler (2001) concluded that while trying hard to maintain their own culture, Asian-American parents were also willing to have children obtain American culture. For her as well as for other researchers (Costigan \& Dokis, 2006; Costigan \& Su, 2004), this parental attitude arose out of a belief in the importance of both sets of cultural knowledge for children's development.

\subsubsection{Home training of academic skills}

A strong point emerging from empirical studies of Chinese immigrant parenting practices suggests that Chinese parents play a very active role in their children's upbringing (Chen, 2001; Gao, 2006; Huntsinger et al., 2000; Lin \& Fu, 1990; Shek, 
1996). The parents not only teach children what is morally and socially correct, but also actively participate in all aspects of their children's learning (Johnston \& Wong, 2002). Although Chinese parenting is traditionally believed to be that of parental control (Lin $\& \mathrm{Fu}, 1990)$, modern researchers claim that "Chinese child-rearing items involve the concept of training" (Chao, 1994, p.1111). Such a statement is gaining popularity in recent studies of Chinese parents (Chen \& Luster, 2002; Gorman, 1998; Xu, Farver, Zhang, Zeng, Yu \& Cai, 2005). While this style of training is based on parental control, it involves parental concern, devotion, involvement and sacrifice as well as close interactions between parents and children (Chao, 1994).

Evidence that a training style is adopted and exercised by Chinese immigrant parents is also presented in Gorman's (1998) study, which illustrated that parents were involved in their children's daily activities and constantly carried out protective and watchful duties. It has been reported that immigrant Chinese mothers exercised constant and strict supervision over their children and were actively involved in their children's lives (Liu, Chen, Rubin, Zheng, Cui, Li, Chen \&Wang, 2005).

In Chinese immigrant families, training is embedded in children's academic studies, starting with preschoolers. It is a common picture that "Chinese preschool children do their home work in the presence of family, all seated around a table" (Huntsinger et al., 2000, p.11). Similarly, Johnston and Wong (2002) noted that Chinese mothers used picture books and flash cards to teach their young child new words.

Another example is Ebbeck and Gokhale's (2004) study that investigated forty Chinese parents in Singapore in terms of their views about children's learning and development in their homes and childcare centres. The parents in that study described the children's academic experiences in the childcare centres as insufficient, with the result that the parents organised private academic tutoring for their children. Huntsinger et al. (2000) observed that Chinese immigrant young children routinely practise the violin, piano, writing or drawing as after-school activities. This is because these "basic skills" were perceived by Chinese immigrant parents to be important for children's development, and the parents felt that "U.S teachers do not assign enough home work" (Huntsinger et al., p.12). Therefore Chinese immigrant parents "often give their children additional work as early as the preschool years" (p.12). Gorman (1998) reported that Chinese 
American parents commented on the insufficient investment of their American counterparts in their roles as parents when they allowed children to do school-assigned work only.

\subsubsection{Parental roles in schools}

Although Chinese immigrant parents actively participate in their children's lives at home, they do not seem to do so in their children's schools (Chao, 1995; Guo, 2006; Li, 2006). Guo (2006), after visiting elementary schools in Canada, reported that the teachers felt that Chinese immigrant parents did not like coming to school. Teachers wondered “why didn't they show up?" (p. 80). Similarly, Guo (2005a), in New Zealand, noted the reticence of Asian parents about participation in their children's early childhood centres.

In a study of expectations of recent Chinese immigrants, Dyson (2001) concluded that cultural and linguistic differences hindered parental involvement in school activities. This could be the result of parents' lack of confidence to work with people of different cultural and linguistic backgrounds (Woods et al., 1999).

Chinese cultural attitudes to teachers serve as another contributor to the level of parents' participation in children's schools. According to Huntsinger et al. (2002), "the traditional Chinese view of education portrays teachers as repositories of knowledge" (p.11). Chinese parents have great respect for teachers and believe that with professional expertise and experience teachers can work well with children in schools. In the context of immigration this is particularly so, because these parents rely on teachers in the mainstream culture to give children a chance there (Adair \& Tobin, 2008; Ulich \& Oberhuemer, 1997). There has been evidence that "Chinese immigrants perceive U.S. public school teachers...to be essential components of a more secure future" (Palmer, Chen, Chang \& Leclere, 2006, p.241). Implicit in these perceptions are two beliefs: teachers can provide children with the essentials to function in the new society and that it is not necessary for parents to intervene in school (Guo, 2005a).

Another important explanation for Chinese immigrant parents' lack of school involvement is their perception that parental responsibilities are within families, which are separate from those of the teachers. Chinese parents did not intervene in their 
children's learning in school but worked directly with children to teach and manage children's learning (Chao, 1995; Chen, 2001; Dyson, 2001; Li, 2006). As mentioned in section 3.1.2.2, in Western countries Chinese parents are reported to engage in much home teaching of their children to supplement the insufficient learning provided by the school.

Furthermore, Woods et al. (1999) reported that immigrant parents were unsure of how to support their children's learning and development with two different cultures. They said that in response to their children's experience with another culture, parents of minority cultures experienced a tension in determining "how far this alternative culture should be encouraged and how far resisted" (p.192). This tension is a key theme in Adair and Tobin's (2008) research of immigrant parents' perspectives. They wrote that "this tension goes to the heart of the problem facing immigrant parents everywhere: How can they raise their children to be able to succeed and feel at home in their adopted country while retaining their heritage language and culture?" (p.145). A reasonable conclusion arising from this tension might be to limit one's participation in schools.

\subsection{Chinese immigrant children in non-Chinese speaking early childhood settings}

\subsubsection{Relationships with peers}

\subsubsection{Forming a bond with peers of the same culture}

The predominant theme that runs through studies on peer relationships of Chinese immigrant children is that they form close relationships with Chinese peers. Feng et al. (2004) found that Chinese-speaking young children formed intense friendship networks in an English-dominant setting. The three Chinese children in their study demonstrated clear behaviours to create and maintain a bond. They concluded that "it is clear that these three Mandarin-speaking children had formed a sub-group as part of their attempt to function in an English-only environment" (p.32). Woods et al. (1999) could explain this phenomenon as these children's reliance on each other to cope with the unfamiliar demands of a new culture. They made the point that the friendships might have their roots in culture and ethnicity. Long (1997) wondered if this could be because samelanguage-speaking peers were able to understand, share and negotiate the meaning of their play experiences together. Furthermore, the social bonding behaviours of these 
children may also suggest that "children from different ethnic groups and socioeconomic backgrounds vary in the extent to which they maintain friendships" (Fletcher, Bridges \& Hunter, 2007, p.1137).

There has been strong evidence that Chinese immigrant children sustained interactions through fluent communications and by adopting socialization themes consistent with what they had experienced in their families (Feng et al., 2004). Feng et al.'s finding is supported by Scourfield, Dicks, Drakeford and Davies (2006) who reported that English-speaking children in Welsh-medium schools only played amongst themselves and spoke their first language.

The basis of children's close bond with peers from the same cultural backgrounds is essentially an explanation of how similar language, backgrounds and learning experiences influence their peer preferences and relationships. Howes' (1996) research on the earliest friendships is a further indicator of how similar play styles help children choose friends. Scourfield et al. (2006) discovered too that familiarities among the children served as a resource to draw on between themselves "especially along the lines of what they felt they 'ought' and 'ought not' to be doing" (p.136). Children prefer being with peers who are similar to them. It has been observed that "children from preschool on tend to like, and play with, classmates of their own race to a greater extent than those of another race" (Aboud \& Mendelson, 1996, p.91).

\subsubsection{Social interaction with peers of the second language group}

In a study of Spanish children in English preschools, Hruska (2000) reported on the social isolation of these children from their local peers. Hruska's research is consistent with the findings of Barnard (2003) and Tabors (1998), which suggested that the limited interactional experiences of second language learners with peers of mainstream cultures was an area of concern.

The reasons cited for this phenomenon are typically twofold: native English-speaking children may not seek out second language speaking peers (Hruska, 2000); and second language learners may have language difficulties (Hruska; Tabors, 1998). Tabors' research found that young second language learners' "linguistic constraints" led them to "social isolation". He described this as a cyclic "double bind of second-language 
learning" (p.22) in which the second language learners' limited social experiences are attributed to their limited skills with the new language, and also contribute to their slow learning of the new language, which again restricts their development of social status in groups.

Emotional barriers of second language learners are of particular significance in terms of understanding their relationships with peers of a new language group (Igoa, 1995). Igoa said that while second language learners are keen to blend in with other children, "their emotions and fears hold them back" (p.38). In an early childhood environment, Konishi's (2007) study of a Chinese girl at an English-speaking preschool similarly noted that "the girl was not ready to join in play with other children... She seldom smiled for anyone at the preschool" (p.268). The withdrawn behaviours of second language learners in English-dominant settings could be an indication of learners' lack of confidence in socializing with English-speaking peers (Barnard, 2003). Due to their low self-esteem and anxiety, second language young learners demonstrate social withdrawal (Hart, Yang, Nelson, Robinson, Olsen, Nelson, Porter, Jin, Olsen \& Wu, 2000).

Krupa-Kwiatkowski's (1998) study provides further examples of second language children's social behaviours in peer-learning contexts. In it, she revealed that learners perceived their social status and that of the people with whom they interacted, and they grounded their social behaviours in these perceptions. Krupa-Kwiatkowski noted that the immigrant child in her study tried very hard to interact with peers of immigrant backgrounds, but displayed restrained behaviours in interacting with children of the mainstream cultural group. For Krupa-Kwiatkowski, this is because the child was typically approached by other immigrant peers but not those of the mainstream cultural group. She described the interaction between the child and the mainstream cultural peers to be "primarily structured through imitative and repetitive activities" (p.160). Unlike Tabors (1998), who attributed social issues of second language young children to their second language limitations, Krupa-Kwiatowski believed that the child's primary concern was with the attitudes of mainstream cultural peers to him.

Krupa-Kwiatowski's belief is supported by Hruska (2000), who claimed that the social experiences of young second language learners were not totally determined by their 
English language proficiency, social skills or personality, but by "the local construction of friendships and the attitudes of native English speakers to new relationships" (p.37). Extending on this perspective, there is evidence to suggest that immigrant children made friends with cross-race peers who included them in common play experiences (Aboud et al., 2003). In the same study, the researchers found that despite explicit samerace preferences of friendships, "cross-race friends, once chosen, tended to be viewed as no different from same-race friends in fulfilling most friendship functions" (p.171). This finding, therefore, alerts us to how the attitudes of children of mainstream cultural groups make an important contribution to the formation of their relationships with immigrant children.

\subsubsection{Relationships with teachers}

In their study of children from diverse cultures, Klein and Chen (2001) noted that the Chinese immigrant children did not seem to like direct informal interactions with teachers. This was especially so for the children from families following strong Chinese traditions. "They [the children] are more accustomed to formal interactions with adults in which there are clear rules regarding responses expected from children" (p.107). Cheng (1987) described young Chinese children's wishes to have a middle person, such as a peer, to communicate with teachers on their behalf. This could be attributed to Chinese children's discomfort with public physical affection (Klein \& Chen).

However, there is also research that indicates immigrant children's eager attachment to teachers during their initial period of settlement. For instance, Igoa (1995) wrote: "in reflecting upon their immigrant experiences, many of my students have mentioned that the teacher is their closest friend at first and is sometimes the only person to whom they can turn" (p.138). She found that immigrant children purposefully sought teacher affirmation in order to develop self value in the new environment. Ozer, Wolf and Kong (2008) claimed that affective relationships with teachers were a core contributor to developing immigrant children's sense of belonging in schools and that these children were longing for it.

It has been pointed out that immigrant children form special relationships with bilingual-bicultural teachers because "these teachers possess cultural mediation skills that can help these students to mediate the two distinct sociocultural environments of 
home and school" (Weisman, Flores \& Valenciana, 2007, p.192). Another explanation for this could be that bilingual teachers establish an environment in which immigrant children perceive themselves as having equal status with non-immigrant peers (Flores, Keehn \& Perez, 2002).

Regardless of Chinese immigrant children's behaviours with teachers, it is unanimously agreed that they are respectful of teachers. In investigating the feelings of ethnically diverse children to schools, Ozer et al. (2008) reported a Chinese girl stating that the most important thing she needed to do in school was be "nice to the teacher" (p.457). Likewise, American Chinese children showed respect to teachers and allowed them to enter their play, although they had rejected other peers under similar circumstances (Feng et al., 2004). Feng et al. explained this as due to the children's perception of teachers' authority and power. This analysis is consistent with the viewpoint that Chinese immigrant children are aware of hierarchies and respect people in authority and with knowledge (Fang, Fang, Keller, Edelstein, Kehle \& Bray, 2003; Yau \& Smetana, 2003). In an attempt to examine learning styles of Chinese learners, Chan (1999) also noted that Chinese learners were raised to avoid challenging teachers because they recognised teachers as a representation of knowledge, power and expertise.

\subsubsection{Learning behaviours}

A recurring theme in much of the research on Chinese children's learning attitudes and behaviour is that Chinese children demonstrate willingness and a desire for learning ( $\mathrm{Li}$, 2004; Trnavsky, 1997). For example, Li indicated that Chinese preschoolers as young as three-years of age, established the purposes of learning in line with their cultural expectations. These children pointed out that learning benefited their personal growth and stated that "I learn and I grow big" (p.126). Li commented that at such an early age, Chinese young children clearly appreciated a commitment to learning. When expressing their perceptions about achievement, Chinese kindergarteners said that "people who make the effort are smart and smart people work hard" (Li \& Wang, 2004, p.417). In analyzing an Asian child's rapid progress in learning, Patary-Ching, Kitt-Hinrichs and Nguyen (2006) inferred that the child's and parents' expectation for achievement motivates the child to try his best in school. 
With regard to play styles, Chinese children prefer different types of play experiences from peers of other cultural groups (Cheng, 1987; Huntsinger et al., 2000; Trnavsky, 1997). Cheng reported that many young Asian children liked physical manipulation of objects but not open-ended symbolic play. This is consistent with Trnavsky's observation regarding Chinese children's limited involvement in social play. Trnavsky found that Chinese children played less but talked more than American peers. For Klein and Chen (2001), it was clear that Asian children liked routine schedules, which they considered to be the result of Chinese children's customary concern over learning outcomes.

\subsubsection{Second language learning and development}

\subsubsection{General views}

In this section, the general views on second language learning and development are developed only from research with an educational focus, as opposed to that with a linguistic focus, because the present research is an educational study.

The development of a second language after the establishment of a first language is referred to as sequential acquisition of bilingualism (Baker, 1996). In this process, psycholinguistic and sociolinguistic factors have been identified as explanations for the outcomes of learners' experiences. According to Mickan (2006), "psycholinguistic and sociolinguistic studies in second language (L2) acquisition have made us aware of the centrality of learners' communicative experiences for learning additional languages" (p.342). Psycholinguistic studies take into account individual differences such as personality, previous language learning experience, language preferences, and cognitive and social strategies or attitudes towards the learning of a new language. Sociolinguistic studies consider the social environment in which language acquisition takes place. Sociolinguistic researchers believe that social interaction constitutes an environment for language learning (Thorne, 2000). Therefore there is alignment between the sociolinguistic views of language acquisition and sociocultural perspectives because they both consider social interactions to be the basis for language development.

Research indicates that sequential second language learners approach learning situations with certain social skills, understandings of conversational rules, ways of influencing communicators and of the social conditions that underpin communication (Krupa- 
Kwiatkowski, 1998). Therefore, what learners most require is 'comprehensible input' (Krashen, 1982). Nowadays, a shift has occurred in the interpretation of language development away from a social learning/behavioural perspective to a more sociocultural perspective (Bruner, 1996). Researchers have started examining not only learners' inner factors and language input, but also the role of teachers, peers and cultural tools in supporting the language learning process (Haworth, Cullen, Simmons, Schimanski, McGarva \& Woodhead, 2006a). These researchers endorse a view that learning a new language calls for an ability to understand culturally appropriate ways of communication across situations. This point serves as a springboard for identifying how social and cultural practices, cultural tools and mediated learning facilitate second language acquisition.

For young second language learners, researchers agree that learning is more likely to occur in playful contexts (Anderson, 2004; Siraj-Blatchford \& Clark, 2001; Tabors \& Snow, 1994), and that children tend to view the new language as a tool rather than a form to learn. Moreover, researchers highlight individual differences of young children as important contributors to their achievement of learning outcomes. They believe that children's personalities influence their second language learning process and for this reason variations in their learning occur (Robinson, 2001). In general, sociable and outgoing children and those who are passionate about participating in the new language activities tend to follow the process with ease. On the other hand, children who are shy, not willing, or have no interest in becoming integrated into the new language groups are in a relatively awkward position when learning that language.

\subsubsection{Language choice/preference}

It has been found that bilingual young children make choices of their language use in accordance with communication situations, communication forms, the role the language serves, and their personal values attached to a particular language (Ledesma \& Morris, 2005). For Ledesma and Morris, even bilinguals who display competencies in both languages prefer one language to another. Both Lanza (1997) and Quay (1993) claim that children as young as two know how to differentiate the use of a language according to the particular languages, the people involved, and the contexts. It is also believed that young learners' language choices and patterns of behaviours are the result of social situations, and in these situations, learners' own needs for communication are the most 
identifiable contributor to their development of a particular language (Lefkowitz \& Hedgcock, 2002; Mickan, 2006). For Lambert (1973), learners' communication imperative is related to their attitudes and motivation. Based on this idea, Lambert constructed a motivational theory to explain what is responsible for learners' level of achievement with a language. Ellis (1985) agrees, saying "learner motivation and needs have always had a central place in theories of SLA [second language acquisition]" (p.11).

As mentioned above, Feng et al.'s (2004) study confirmed the use of the Chinese language by Chinese speaking young children in an English-dominant setting, which they attributed to the children's understanding of the usefulness of it to form and maintain friendships. Speaking their first language was a strategy for these children to gain entry into a common play activity because "the use of their first language seemed to establish a type of social and emotional bonding that was unique to these children" (p.24). Feng et al. noted that once the children started using their first language, they stuck with it and did not move to the dominant language. The study by Scourfield et al. (2006), cited earlier, also pointed out that English-speaking children spoke English among themselves in Welsh-medium schools, regardless of how well they knew Welsh.

There is evidence that for emotional security, children used their first language with their first-language speaking peers in a second-language learning setting (Feng et al., 2004). This is because in a new linguistic environment, young children might "feel silly, humiliated, and helpless", therefore they might have found it necessary to be together to speak in Mandarin so "they did not have to work hard communicating with other children on unfamiliar topics and experiences" (Feng et al., p.33). Feng et al. understood that the first language use allowed the children who could not speak English well to socialise with their peers, and also fulfilled the desires of the children who could use English to have extended and rich exchanges with others. Using the first language helped second language young learners develop a sense of belonging, safety and selfesteem in a new learning environment (Feng et al).

From another angle, Saunders (1988) considered peer pressure to be a possible contributor to the choices of language learners, because the purpose of pleasing peers 
could result in their exclusively using one language with each other in a bilingual environment (Lefkowitz \& Hedgcock, 2002).

Evidence of selective use of first language also came from Reyes (2004) who observed Spanish-English bilingual children employing their first language, Spanish, when engaged with a difficult task. This surprised Reyes as the children were all competent English speakers. Reyes concluded that in response to cognitive challenges, children could gain comfort from their first language. These findings were also reported in Wang and Pape's (2007) study of Chinese boys in English learning settings where the boys predominantly used English when playing together but changed to Chinese when encountering difficulties with certain English words.

Selective use of languages by second language learners has gained prominence as a research interest in the area of second language discourses (Ledesma \& Morris, 2005; Reyes, 2004). This phenomenon is linguistically termed as code switching, which, according to Jorgenson (2003), is a behaviour closely associated with language choice patterns.

Two types of code switching have been proposed. They are "situational shifting" and the "linguistic change of code" (Beardsmore, 1986, p.49). When people switch from one language to another in response to the change of speaking topics, settings or speakers, they make a situational shift. If the language change occurs at the level of sentences, phrases or words, this is called a linguistic change of code.

Among all the recent discussions on code switching, there are two viewpoints. One perceives code switching as a sign of language incompetence (Baker, 1996), and the other understands it as a strategic method of communication. Baker claims learners' limited proficiency in one language is a contributor to their application of some words of the second language.

There is a large group of researchers, however, who understand code switching as a strategic form of communication. For example, Reyes (2004) proposed that code switching was a strategy to extend communication, but not a sign of language incompetence. He believed that even young children know how language functions in 
sociocultural situations and they can manipulate the language in a wide range of communication contexts, including the topics of conversation and the language abilities of their peers. Reyes concluded that "children during peer interaction use the language with which they both feel most comfortable and have greater competence" (p.93). Code switching is considered to be selected and operates "as a fairly conscious device" (Beardsmore, 1986, p.77). Canagarajah (1995) made a similar argument from his study of Tamil children learning English, in which he reported that the children used English for class related formal tasks but Tamil in interactions "that [were] considered, personal, personalised, unofficial or culturalised" (p.190). When they could speak both languages, children alternated the use of the languages in accordance with the speakers and the contexts. Haworth et al. (2006a) echoed this finding in their study on Samoan children who were learning English, and said that "these children drew on all of their available language, both Samoan and English, in the process of making meaningful conversation" (p.305). Many other researchers are in agreement with this view, claiming that code switching is a sign of language competency (Alejandro \& Celeste, 2001; Hughes, Shaunessy, Brice, Ratliff \& McHatton, 2006; Kohnert, Yim, Nett, Kan \& Duran, 2005).

Another way of understanding language mixing within a sentence is to see it as a means for fulfilling speakers' desire for linguistic creativity through verbal interactions (Bhatia \& Ritchie, 2008).Communication using two languages represents the flexibility inherent in languages themselves. Although languages usually function as separate linguistic systems, they can become a joint device for a creative style of communication. As Bhatia and Ritchie have commented, "the cooperation... and coexistence of the bilingual's two languages make a bilingual a very complex and colourful individual" (p.10).

\subsubsection{Learning strategies}

Research has documented that sequential second language acquisition is a purposeful and strategic process in which learners demonstrate certain behaviours and adopt strategies (Baker, 1996; Wang \& Pape, 2007). Ellis (1985, p.164) describes strategies as either learning or communication strategies, and aligns them with two types of second language knowledge: "declarative and procedural". Learning strategies are the "declarative knowledge about 'knowing that', while communication strategies are associated with the procedural knowledge about 'knowing how"'. Learning strategies 
are the processing of new language data for acquisition and use, and are usually unobservable and related to language reception. Communication strategies require behavioural modifications to use the new language knowledge in conjunction with other knowledge sources to communicate in it. Communication strategies are typically visible and related to language production.

'Being silent' appears to be the most identifiable learning strategy adopted by young second language learners (Krupa-Kwiatkowski, 1998; Siraj-Blatchford \& Clark, 2001). It is employed by children refraining from interactions with speakers of the new language. Although learners during this phase may not be completely silent, they minimise their conversations with people who speak the new language.

Two possible reasons have been put forward for the 'silent' phenomenon in second language acquisition: the first being that the children "become aware of the impossibility of using the first language to make sense to the second-language speakers" (Guo, 2005b, p.39), and the second is that some learners are "inner directed" (KrupaKwiatkowski, 1998, p.135) that is, for them it is better to "approach language learning as an intrapersonal task" instead of "an interpersonal, social task". Usually, during the silent period, children watch and listen carefully in preparation for producing new language items (Siraj-Blatchford \& Clark, 2001; Tabors, 1998). This silent way of learning means the children are adopting a memorizing approach. Ellis (1985) takes the view that although memorization is unheard or unseen, it is often used by second language learners to learn new language expressions or language patterns. KrupaKwiatkowski wonders if the adoption of these unobservable strategies indicates that the "language that is eventually used with the target language speakers...is first arduously practiced in safer, less threatening environments" (p.171).

In discussing silence as a learning and communication strategy for second language acquisition, it is important to note other perspectives that view it as an indicator of a learner's social identity and a response to learning situations (Brown, 1979; Granger, 2004; Nakane, 2007). Granger understood the silent period in some second language learners to be a process of self discovery, and she argued that it could indicate "the loss, ambivalence, and conflict that accompany a transition between two languages, a psychical suspension between two selves" (p.62). This statement highlights silence as a 
sign of the complexities between languages and identities that are embedded in the dayto-day experiences of second language learners. Such a view adds a new dimension to our understanding of silence in young children's second language acquisition.

Communication strategies that are commonly used by second language learners are “avoidance of $\mathrm{L}^{3}$ rules, [of] certain speech functions such as replacing a topic, using a language instead of the new language, replacing one L2 form with another, using body language, overtly requesting assistance, waiting for the item to come, and thinking of the form in another language and then translating it into the L2" (Ellis, 1985, p.184).

In addition, Ellis (1985) recorded that difficulties in responding to communication tasks in second language situations led young learners to use a language resembling the first language, or a language resembling the second language. The language resembling either of the two languages according to Krupa-Kwiatkowski (1998) is "an invented language" (p.168). Behind the use of this strategy is the user's intention to compensate for the second language shortcomings or to play with the languages. KrupaKwiatkowski proposed that although children understood that their invented language did not make sense to others, they used it to cope with the new learning situation, as well as to gain personal pleasure.

Other strategies that second language learners have been reported to employ are: joining a group, acting as if they understand what is going on even if they do not, and using a few carefully selected words to speak (Fillmore, 1976). Moreover, Krupa-Kwiatkowski, cited earlier, identified the learner using "mediation strategies" (p.143) which usually involve asking a familiar adult to make the intentional move and have him/her included in a group.

Imitation and repetition are also communication strategies reported to be widely used by young second language learners (Ellis, 1985; Patary-Ching et al., 2006; Tabors, 1998). Unlike memorization, which is to quietly memorize the new language features, imitation and repetition involve deliberate copying of utterances used in a speech.

${ }^{3}$ L2 means second language. 


\subsection{Working with immigrant children: The role of teachers}

Working with immigrant children is a challenging task for teachers of mainstream cultures (de Melendez \& Beck, 2007; Fumoto, Hargreaves \& Maxwell, 2007). Because of the cultural differences between them, teachers could have "the experience of feeling as an outsider" (Marx, 2008, p.38). Marx examined the perspectives of four white teachers about their work with non-white children, and found that "all teachers admitted that there were many ways in which they could not relate to their students. All of those were connected obviously or subtly to race" (p.55). She concluded, "Whiteness was a limiting characteristic for all teachers" (p. 59). This view is supported by Adler (2001) who wrote that "even whites who have held true to our calling as educators continue to struggle with the issues of white dominance" (p.265).

Language differences are identified as another major barrier in teachers' work with immigrant children. Fumoto et al. (2007) reported:

It can be extremely difficult to ascertain whether these children's problems .... are due to their limited experience in English language or due to their learning difficulties in general. This can be a source of enormous anxiety for many teachers. (p.137)

The barriers can be compounded when teachers have to consider the needs of each individual learner in large group situations (Fumoto et al., 2007). Brooker (2005) admits that "none of us will under-estimate the difficulties facing teachers with large groups, too little help, and too many children speaking an unknown language" (p.128).

Despite the challenges, research demonstrates that teachers must play an active role in immigrant children's lives. For example, Dalli (2000) reported on children and parents' experiences when starting childcare in New Zealand, that "what teachers did, as well as what teachers did not do, made a difference".

For the purpose of this literature review, identifying teachers as important in immigrant children's development serves to identify them as essential support in these children's learning experiences. As de Melendez and Beck (2007) state: "teachers are the key to successful multicultural education" (p.vii). 


\subsubsection{Understanding and positively responding to differences}

The literature on teachers' support of immigrant children indicates teachers' construction of theoretical and practical concepts of these children's needs and strengths. Schofield (2007), after researching teachers' work with immigrant children in New Zealand claims, "It is important first of all for teachers to understand the needs of immigrant children. Based on this knowledge, teachers can support immigrant children" (p.24).

In much of the research, a recurring idea in relation to supporting children of diverse cultural and language backgrounds has been acknowledging, welcoming and positively using their differences (Robinson \& Jones-Díaz, 2006). This message suggests that working with these children revolves around differences. Both Lindon (2006) and de Melendz and Beck (2007) state that teachers cannot take a colour blind perspective but should face the reality of diversity and reflect on diversity within culture. This statement implies that teachers can neither ignore differences nor think there are none among children, because differences are salient features of human beings and should be responded to in visible ways.

Mickan (2006) particularly recognises teacher behaviours as a significant influence on children because children usually observe what they do so they can comprehend how to behave in a new learning environment. Ideas regarding teaching behaviours typically point to teacher reflections on their own cultural attitudes. It is necessary for teachers who work with children of minority cultural groups to raise cultural awareness and to scrutinise possible biases (Lindon, 2006). Teachers should make their respect for cultural identity visible, because in doing so, they convey a message to all the children that it is acceptable to be different. Lindon argues that young children do not initially associate people's skin colour or group differences with their abilities but develop this attitude from those of adults.

\subsubsection{Using speaking approaches}

To support young language learners, teachers should use the following speaking approaches with them: visual cues; simple and slow speech; code switching in instructions; body language; repetition; reiteration; emphasizing certain words; checking understanding; prompt answering of questions; helping with answers; and relating the 
conversations to what the child knows and to what is happening (Alejandro \& Celeste, 2001; DeBey \& Bombard, 2007; Mickan, 2006). Patary-Ching et al. (2006) state that teachers should help children express ideas by providing them with the words needed when conversing with them. Code switching of young children should be encouraged and recognised "as a third legitimate code" (Hohnert, Yim, Nett, Kan \& Duran, 2005, p.259). Alejandro and Celeste extend this by saying that "a code mixed combination of the two languages constitutes a unique third language form. Educators [who are able to do so] should consider its use in instruction" (p.13). In view of the silent behaviours of some children, Klein and Chen (2001) tell teachers to allow children to be silent and not to pressurise them to talk. These arguments clearly imply that teachers should make themselves understood and the conversations should be meaningful for young second language learners. Underlying this is that teachers understand the language levels of the learners and their learning needs and interests (Alejandro \& Celeste).

\subsubsection{Supporting children's development in a new language and culture}

Inherent in the learning of a new language and culture is making sense of the new cultural context (Patary-Ching et al., 2006). It is important that learners are socialised with the second language culture, and supported to use the new language for making meaning in cultural practices (Mickan, 2006).

In an attempt to investigate successful bicultural development Mickan (2006) places an emphasis on routinised cultural practices as the key to help children succeed in learning a second language and culture. This is consistent with a point made in an earlier study by Ellis (1985), who perceived repeated interactions to be very useful for learning a new language and culture. The explanation for this is that "routines contained much of the cultural information and language the children would need to gradually become competent members of the class" (Willett, 1995, p.485). These ideas are firmly aligned with the theoretical concept of 'learning through participation in cultural practice' constructed by Rogoff (2003).

In recognition of the complexity of second language and cultural acquisition, Haworth et al. (2006a) take the view that it is not only routines that need to be addressed. The entire learning programme should be designed and implemented to enable children to utilise all their learning resources. They suggest a balance between freely chosen play 
experiences and organised opportunities for young second language learners to interact with teachers and peers.

Regarding young second language children's social needs, Tabors' (1998) study suggests that the social isolation of these learners from their English-speaking peers might be due to English-speaking children's lack of awareness of these children's difficulties. He asserts that "English-speaking children do not understand why a particular child is not eager to join their play, so they leave the child out of the group" (p.25). In response, teachers should intervene in the play of children who are socially isolated by purposefully pairing the child with others (Tabors). This could be done by organizing "cooperative problem-solving tasks" or "paired activities" (Haworth \& Haddock, 1999, p.13). Teachers should explain to English-speaking children, especially those who are not happy to team up with second language children, that this child comes from a home where he/she has learned another language. This explanation will help English-speaking children develop understanding about that child's difficulty (Tabors). A buddy system can be set up in class and teachers can enlist some outgoing Englishspeaking children to help with this (Siraj-Blatchford \& Clark, 2001). Moreover, teachers can engineer the seating arrangements at group times to make these Englishspeaking children sit together with a second language speaking child, which should help the young learner quickly make social contacts (Tabors). Even so, Konishi (2007) warns that teachers need to develop awareness of second language children's social preferences in their attempt to scaffold these children's peer experiences because the children can have particular social needs aligned with their home cultures.

In an ethnographic study, Willett (1995) reported that if English-speaking children were more open to friendships, non-English speaking children could be more able to develop group status, despite their inability to communicate well in English. de Melendez and Beck (2007) extended this by saying that in multicultural classrooms, it is important that children are supported to be learning with each other by discovering that they can all contribute.

\subsubsection{Implementing multicultural practices}

It is suggested that multicultural learning environments should be established through using children's home and previous experiences (Podmore, Sauvao \& Mapa, 2003; 
Woods et al., 1999). However, given the examples in which multicultural practices are exercised superficially using "folksongs and folktales, food fairs, holiday celebrations, and information about famous people" (Sleeter, 1995, p.23), it is important that multicultural education does not consist of only a list of children's home items to be used in practice, but is infused into mainstream teaching programmes and implemented through a variety of teaching approaches (Brown \& Kysilka, 2002; Sleeter \& McLaren, 1995). Moreover, because school cultures often reflect the culture of their teachers but not the culture of the children (de Melendez \& Beck, 2007), a key to meaningful cultural practice for minority children are teachers' reflections on the influence of their own cultures on their teaching practice. It is important that teachers put aside the cultural resources or tools that they prefer to use, and always apply those of the children's.

A tool that has been highlighted as important for children of minority cultural groups to develop in another cultural setting is their first language (Baker, 2006; Faust \& Smidt, 1998; Podmore, Samu \& the Ao'oga Fa'aSamoa, 2006). Inspired by sociocultural theories that highlight the leading role of language in the development of thought, researchers currently agree that first language maintenance is crucial for bilingual children because learning originates from home, and children develop essential sociocognitive thoughts via home language and culture. Alladina (1995) perceives the learning of a new language as not difficult if the foundation in a home language is strongly laid, because the process for learning both languages is very similar. Faust and Smidt in a study of Samoan children's learning in an English-speaking childcare reported that the use of the Samoan language as a learning tool helped the children settle into the programme, which ultimately contributed to their learning of the new culture and the new language.

In terms of supporting the use of first language in a second language speaking early childhood setting, researchers believe that the most essential aspect of a new language learning context is the legitimacy of the first language (Hohnert et al., 2005). Implicit in this view is that young learners are encouraged to use the home language in a learning programme that accepts its value. 


\subsubsection{Developing partnership relationships with immigrant parents}

The significance of developing partnership relationships between teachers and parents of different cultural backgrounds to provide their children with meaningful learning experiences has been highlighted by many researchers (Faust \& Smidt, 1998; Haworth et al., 2006a; Haworth et al., 2006b; Klein \& Chen, 2001; Lindon, 2006; Podmore et al., 2006). At the same time, it is also acknowledged that working with parents of diverse cultures is challenging for teachers, mainly due to the difficulty of achieving effective communication with them (Kaufman, 2001), different cultural expectations and practice (Gonzalez-Mena, 2008), and lack of time and limited opportunities (Martin, 2006).

Some literature reviews have suggested that a key to teachers' work with parents of other cultures is building and maintaining parents' confidence in their role in children's schools or childcare settings (Hoover-Dempsey \& Sandler, 1997; Tett, 2001). The specific strategies suggested in literature include: explicitly welcoming the parents and leaving them with a good impression during the first visits; having open communication about sharing the care of children; defining success from parents' perspectives; approaching and contacting parents in a way that they are comfortable with, either in the centres or by visiting their homes; helping with the language; providing them with daily access to the people whom they can talk with; providing opportunities for parents to see their children work in the environment; maximizing cultural courtesies; learning to address parents; and providing opportunities for parents to be involved (Brooker, 2005; Kaufman, 2001; Klein \& Chen, 2001). In addition, the necessity of maintaining first languages for second language young learners particularly highlights the importance of teachers' work with parents. The development of trusting relationships with parents enables teachers to help parents understand the necessity of maintaining their first languages for their children, because there are cases in which parents encourage children to use the second language at the expense of their home languages, from the mistaken belief that the two languages have different purposes and they are not connected (Woods et al., 1999).

\subsection{Chapter summary}

This chapter has reviewed literature on Chinese immigrant children's learning and development in homes and EC settings. Common themes that emerge from literature on children's learning experiences in Chinese immigrant families include the pervasive 
power of Confucianism in guiding Chinese people, and the inevitable influence of the new living dynamics on Chinese immigrant parents. A close examination of literature on Chinese immigrant children's learning experiences in non-Chinese speaking EC settings reveals themes of English acquisition, learning behaviours, and social experiences with peers and teachers. There is also increased recognition of the importance of teachers supporting Chinese immigrant children's learning and development in early childhood settings.

To summarise, Chinese immigrant children enter into non-Chinese speaking EC settings equipped with their family-oriented learning experiences such as the use of their first language, a desire for learning, preferences for making friends with Chinese peers and a tendency to respect teachers. There is also evidence that during the course of development in new language and cultural learning contexts, immigrant children demonstrate their need to take up available cultural activities in the new settings, to make friends with peers of the mainstream culture and to communicate in the new language. Despite the fact that none of these studies specifically focused on the experiences of Chinese immigrant children in non-Chinese speaking EC settings, the literature points out the importance of family cultural experiences to facilitate their learning experiences in another cultural community. As they develop in an out-of-home setting, children construct new knowledge, new positions and new cultural meanings in line with the contextual insights of the new setting and the knowledge and experiences of their families (Brooker, 2002; Rogoff et al., 2007).

The following chapter moves to the empirical part of the study. The focus of that chapter is to outline my methodological choices for the field investigation of this research. 


\section{Chapter 4: Research methodology and research design}

\subsection{Introduction}

This chapter describes the methodological framework underpinning the research design, encompassing qualitative methodology derived from the conceptual basis of the research, the phenomenological paradigm that provides the position of inquiry, a multiple case study approach as a specific practice for making inquiries, and the research methods to gather data. This framework focuses on exploring Chinese immigrant children's learning experiences in New Zealand EC centres to answer the research questions as set out in Chapter 1.

\subsection{Methodological choice: Qualitative research}

This study aims to describe and explain a phenomenon: the learning experiences of Chinese immigrant children in New Zealand EC centres. Through understanding this phenomenon, the study intends to gain insights into how Chinese immigrant children could be supported in their learning and development in New Zealand EC centres. For me, the most important feature of this study is the direct learning experiences of Chinese immigrant children and the meanings associated with them. Investigating lived experiences and understanding the meaning constructed around them is consistent with a qualitative research approach. This is because qualitative research methodology seeks to gather rich information about people's activities in natural settings through building on insiders' perspectives (Denzin \& Lincoln, 2005; Maykut \& Morehouse, 1994). According to Gay, Mills and Airasian (2000) "qualitative research seeks to probe deeply into the research setting in order to obtain understandings about the way things are, why they are that way, and how the participants in the context perceive them" (p.16).

\subsection{Position of inquiry: Phenomenology}

A position of inquiry is an overarching perspective that shapes people's stance about research, and phenomenology is a major position of inquiry in qualitative research (Dahlberg, Drew \& Nystrom, 2001). As Bryman (2004) argues, the attempt to see through the eyes of the participants by qualitative researchers aligns them with the position of phenomenology. 
A phenomenological position describes, interprets and seeks to understand how individuals experience phenomena. Phenomena are the appearances of things, the ways people experience things and the meanings things have in people's experiences (Dahlberg et al., 2001). Phenomenology is rooted in the assumption that "there are many ways of interpreting the same experience, [but] the meaning of the experience to each person is what constitutes reality" (Ary, Jacobs \& Razavieh, 2002, p.447). According to Bryman (2004, p.14), "phenomenology views human behaviour ... as a product of how people interpret the world". The application of a phenomenological perspective, thus, allows researchers to explore conscious experience from the first person point of view.

Of particular importance in phenomenological research is researchers' attention to the meanings of the experiences being studied (Bryman, 2004). According to Edmund Husserl (1931/1977), the founder of phenomenology, behind any phenomenon is 'intentionality', which is one's directed consciousness about an event. In this view, a phenomenologist intends not only to see how, but also why experiences become experiences for the participants. As Schultz, Walsh and Lehnert (1967) highlight, the combination of identifying and understanding meaning is vital to achieving the aims of phenomenological research.

Lincoln and Guba (1985) note that phenomenological research recognises the interdependent relationship between the knower and the known. Associated with this is the researcher's own subjective process as a resource to investigate phenomena. Two phenomenological tools, introspection and verstehen are related to this research process (Wagner, 1970). Introspection is the researcher's own reflection on a research process; verstehen, meaning understanding, refers to the researcher's effort to understand the experiences of the researched. According to Dahlberg et al. (2001), the task of phenomenology is to make manifest the intersubjective nature of the human world. Through building interrelationships in research, phenomenology shows a close link with sociocultural theories which promote the inseparability of individuals and between individuals and their context (Brennan, 2006).

Phenomenological researchers who conduct research with children invite children's perspectives of their life experiences in order to understand these experiences. Danaher 
and Briod (2005) state that although infants and young children might have limited communication competence or skills, phenomenological researchers should not underestimate them but attempt to recognise and include children's voices.

These insights influenced how I gathered information from the children in this study. In an attempt to investigate their learning experiences, I sought the children's own understandings of these experiences. So it was important that I learned about the experiences of the children, not only by observing how the experiences appeared, but also by listening to the perspectives of the children. I took the view that to understand the experiences of the children I should build relationships with them and engage in considering how, as an observer and enquirer, I experienced my role in this investigation process. Denzin and Lincoln (2003) claim that researchers should hear their own voices alongside the voices of research participants because rich and in-depth information from multiple sources characterises qualitative research. This is why I made a reflexive statement and established my own position in this research (see section 1.4), to show how my presence and positioning might have influenced the research process and its outcomes.

It is important to note that the use of phenomenology in this research was to enable it to gather the perspectives of the children, their parents and their EC teachers. Through working with the participants in a reflective way, and entering into their perspectives through conversations and interviews, I could obtain insights into the meanings that they brought to their individual actions. Meanings thus became shared and connected. They arose from a complex interplay between the beliefs and practices of a group of people about their experiences as, and with, the Chinese immigrant children in this study. Phenomenology, in this sense, was not compared with sociocultural theories but rather used to complement them because it served as a springboard for identifying the sociocultural and historical sources of the children's learning and development.

\subsection{Research practice for making inquiry: Multiple case study}

Denzin and Lincoln (2003) note that qualitative research does not have a distinct set of practices that are entirely its own, so that a researcher should make the methodological decisions and identify approaches and strategies of inquiry that are useful for the 
research. In my research, the specific research approach that I chose to use for data gathering and analysis was that of qualitative case studies.

Qualitative case studies are frequently used when an attempt is being made to understand a phenomenon through a detailed and comprehensive study of one or a small number of cases or instances. The case or instance can be "a program, an event, a person, a process, an institution, or a social group" (Merriam, 1988, p. 9). Anderson (1998) notes that a case study allows a researcher to analyse a phenomenon in a holistic way through the use of many sources of evidence. It aims at "an interpretation in context", to "uncover the interaction of significant factors characteristic of the phenomenon" (Merriam, p.10). Bell (2002) believes that the greatest strength of case studies lies in a researcher's concentration on the specificity and the details of various interactive processes of the instance or situation, thereby portraying a full picture of a phenomenon.

Merriam (1998) identifies three essential characteristics of qualitative case studies, including being "particularistic, descriptive and heuristic" (p.29). She explains that qualitative case studies give an in-depth description of a particular instance, which reveals in detail the meanings associated with this instance. The discovery of new meanings often leads to some rethinking of the instance. Because of this, case studies are grounded in an inductive mode of reasoning.

To conduct case studies Tellis (1997) highlights the need for researchers to watch for people's interactions. He considers a salient feature of case studies to be a consideration not only of those under investigation but also the relevant groups of others with whom a participant has interactions. The commitment to social factors of the case study approach, therefore, brings to life the emphasis of sociocultural perspectives.

The aim of my study, which is to describe and explain the learning experiences of Chinese immigrant children in New Zealand EC centres, sits comfortably with the case study approach. The phenomenon under study is the learning experiences of these children. My aim is to search for rich and in-depth evidence about these experiences. Moreover, my intention of identifying educational implications for working with Chinese immigrant children through understanding their learning experiences 
necessitates the use of the case study approach. The inductive mode of reasoning of the case study approach can add a useful dimension in helping to discover new meanings based on the understanding of cases.

An important purpose of this research is to introduce readers to a group of Chinese immigrant children and their learning experiences. Although the data are presented in an overall pattern as one phenomenon across all the children, it is initially analysed thematically for each individual child (See the example of a case report in Appendix 10). The findings of this study are generated in two steps: gaining a deep understanding of the unique features and context of each case; developing an understanding of common themes across them. The study contains eight individual cases, therefore I refer to it as a multiple case study (see section 4.6.2.5.2).

The following table outlines the inquiry framework of this study, as described in the sections above.

\section{Table 4.1: Inquiry framework and methodological rationale}

Inquiry choices

Methodological choice Qualitative research

\section{Position of inquiry} Phenomenology

\section{Practice of inquiry Case study}

There is congruence between qualitative research and the aim as well as the theoretical underpinning of this study, such as the sociocultural perspective. Qualitative research is naturalistic, descriptive and in depth, aims to capture meanings and is concerned with the process of research. My study of Chinese immigrant children aimed to describe and capture the meanings of their life experiences. In line with a qualitative approach, I am able to gather rich and detailed information through exploring sociocultural activities and events in Chinese immigrant children's living contexts.

Phenomenology strives to take an intersubjective perspective and to allow experiences to emerge in a way that the people in the experience see them as meaningful. It accepts observable matters and gives prominent recognition to the voices of the researched. In my study, phenomenology ties together the apparent phenomenon of what children do with a sociocultural underpinning of why they do it, thereby satisfying the need to capture not only the experiences of these children but also the meanings of their experiences within their sociocultural contexts.

The case study approach is consistent with the aim of my study of collecting rich information about a particular phenomenon, in this case, Chinese immigrant children's learning experiences. The learning experiences of Chinese immigrant children as a phenomenon comprise many instances and my purpose to gain in-depth insights of these instances can be served by a multiple case study approach. 


\subsection{Methods of inquiry}

The methods of inquiry were chosen to address the research questions in line with the conceptual framework outlined in the sections above.

\subsubsection{Participants}

The selection of the participating children followed four main criteria. Firstly, they had to be young children aged 3 to 5 years who spoke Mandarin, standard Chinese as their first language. Secondly, the children or their parents had immigrated to New Zealand from China, Hong Kong or Taiwan within the last ten years. Thirdly, the children attended an education and care centre in a major city of New Zealand at the time of my study. Lastly, the children consisted of an equal number of boys and girls.

There were issues to be considered during the selection process. The first issue was the choice of the age group of the children. I chose only young children (aged 3 to 5 years) rather than infants and toddlers because young children would have had longer experiences in their families than infants and toddlers. Since the research focused specifically on Chinese immigrant children, it seemed likely that the study would yield more meaningful data if conducted with those who had been immersed for some years in their family cultures.

In addition, in view of the ongoing influx of Chinese new immigrants into New Zealand, studying recent immigrants was important as they would be likely to uphold more culturally-related childrearing beliefs and practices than those who had been in New Zealand for longer.

Two ideas informed my selection of education and care centres as the research sites. Firstly, education and care centres have the highest participation rates of Asian children among all licensed early childhood services. The latest data (Ministry of Education, $2009 \mathrm{~b}$ ) show that 7,730 Asian children were enrolled in education and care centres out of a total of 12,357 Asian enrolments in all types of EC services. This number was significantly higher than the 3,689 enrolments of Asian children in kindergartens, which were the second most attended type of EC services for these children. Secondly, most education and care centres provide full day services, allowing children to have continuous all day experiences. The data gathered in these settings would be more likely 
to provide information about Chinese immigrant children's daily learning experiences than in sessional settings, such as kindergartens.

I decided to have an equal number of boys and girls participating in the study because I aimed at portraying an overall picture of Chinese immigrant children's experiences in New Zealand EC settings. A focus on any particular sex would not achieve this aim.

The initial search for suitable participating centres for both the pilot study (the first phase) and the main study (the second phase) occurred through consultation with the local telephone book. A list of licensed education and care centres within easy travelling distance from my place of residence was noted and the first contact was made by phone. I telephoned all the listed centres to check their interest in participating in the study and the availability of Chinese immigrant children who could meet my selection criteria.

This research was conducted in two phases, the pilot phase and the main study phase. For both phases of the study, once the initial contact and the approval process was completed in the centres, the centre managers or supervisors were asked to identify suitable children who met the criteria. At the same time, the managers and supervisors also made a request within their teaching teams for volunteers in this study. Selection of the teacher participants was based upon the following three factors: an interest in the study focus; an intention to know more about Chinese immigrant children; the experience of working with the chosen child/ren. Following that, I sent information letters written in English to the prospective participating teachers and in Chinese to prospective participating parents. The letters explained the aims, background, timescale and data gathering process of the study. The participation of both parties was formally invited in the letter.

Two EC centres participated in the pilot study. Using the selection criteria, one child from each centre was chosen for the study. In total, two Chinese immigrant children, two Chinese immigrant mothers and five EC teachers were included in the pilot study.

During the main study, the first round of the phone contact also resulted in two interested centres. Two parents from one centre agreed to participate and to have their children included in the study. At that time, one parent from another centre also gave 
permission. Because the study aimed at exploring in detail the learning experiences of each child, these children's experiences were studied one after another. Upon completion of the first three investigations, I contacted other centres, following the same process and had four more centres participate. Among them, one centre contributed two children and the other three centres one child each.

The total number of participants in the main study were eight Chinese immigrant children, ten Chinese immigrant parents and seventeen EC teachers. Among all the teachers who participated in the research, three were of Chinese ethnicity, who could speak Chinese. The other fourteen teachers all declared themselves to be New Zealanders of European descent, with one having some Pacifika ancestry. These teachers could only speak English. All the participants lived in a major city in New Zealand. In two centres, all teachers were New Zealanders of European descent and in the other four centres, there were teachers from Indian, Maori and Pacifika cultural backgrounds. In all the six EC centres, most of the children were of New Zealand European ethnicity, but there were also children from Chinese, Indian, Korean, Maori, Pacifika, and European background families. Table 5.1 summarises details about the participating children's background and centre context.

The size of the participant group was designed to allow me to concentrate on each participating case and to document rich information to address the aims of the study. For this study, the depth of the data, my documentation, and analytical abilities were important considerations. Involving participants at a manageable level was, therefore, an important decision that I needed to make in order to meet the expectations for the quality of this research, as well as achieve them within the timeframe.

\subsubsection{Data gathering instruments}

I designed the study to obtain rich and detailed information about Chinese immigrant children's experiences. To achieve this end, I used two major methods of data collection for each case study. One method was my field notes encompassing direct observations of each child's behaviour in an EC centre alongside my reflective field notes containing insights, understandings, working hunches, questions and thoughts generated during the data collection process. The second method was to conduct interviews with each child, the parents and early childhood teachers. 


\subsubsection{Field notes}

The use of field notes was grounded in a qualitative research methodology and was aligned with case study approach aimed at obtaining live and detailed information about the learning experiences of the children in this study. I intended to document detailed episodes of what was happening in Chinese immigrant children's lives in EC centres through a written account of what I heard, saw, thought and experienced in the course of collecting and reflecting on the data. Field notes have been given great recognition in qualitative research and, as Bogdan and Biklen (2003) claimed, the achievement of a successful outcome of qualitative research depends on "detailed accurate and extensive field notes" (p.111).

My field notes encompassed two parts: a descriptive part and a reflective part.

The information recorded in the descriptive field notes included "portraits of the subjects; reconstruction of dialogue; description of physical setting; accounts of particular events; depiction of activities; the observer's behaviour" (Bogdan \& Biklen, 2003, p.113-4). In particular, I wrote down the social interactions in which each study child participated, and play and learning activities the child engaged in. During the observations, I paid special attention to the children's use of language, the situations in which they spoke, the people involved, and the body language the children displayed when they spoke. I used pen and paper to record my observations and a cam video recorder to document salient scenarios, on the basis of their clear illustration of the children's language, behaviours or social relationships.

The reflective part of my field notes recorded my personal experiences and accounts of the research journey. They included "reflections on analysis; on method; on ethical dilemmas and conflicts; on my frame of mind; on points of clarification" (Bogdan \& Biklen, 2003, p.115-6). After returning from each field visit, I wrote about my feelings, ideas, impressions and experiences associated with the actual actions described. The interpretations were made in spaces adjacent to what was recorded as descriptive notes. Additional thoughts and experiences were recorded on separate pages to accompany the rest of the field notes. 


\subsubsection{Interviews as conversations}

Kvale (1996, as cited in Dahlberg et al., 2001, p. 154) valued interviews in research by questioning "if you want to know how people understand their world of life, why not talk to them? This question supported my use of interviews which sought to gain an understanding of people's lived world. As stated in Chapters 2 and 3, my adoption of sociocultural theory meant that although the study involved Chinese immigrant children, their lives were inevitably related to others, particularly their teachers and parents. Therefore, the voices of all these people needed to be included.

\subsection{Child interview}

My decision to use child interviews in this study closely aligned with the philosophical orientation of phenomenology. The aim was to obtain children's own perspectives and interpretations of their experiences. The significance of seeking children's voices in educational research has been clearly acknowledged (Powell \& Smith, 2006; Smith, Duncan \& Marshall, 2006). Of particular importance, according to Robbins (2002) is not to focus on the topic of interest to the researcher, but to have extended conversations with children and during the interview, to use the same style of cultural tools that children themselves might use. In this way, researchers can gain "a greater insight into their [children's] thinking and the activities in which it is embedded" (p.13). In Robbins' words, it is to take "a sociocultural approach to interviewing young children" (p.13). Therefore, this process cannot be too formal but should take the form of conversations.

In each interview with the children, I used our common first language, Mandarin Chinese, as the key tool for our conversations and the process was supported by five stories constructed around five typical life experiences of children (Appendix 6). The topics of these stories included being at home for a day, starting the day at the early childhood setting, free play time, playing with peers, and playing with a teacher. Using these stories, I showed the child a picture created for each story to tell only the beginning of the story and asked the child to continue it in his/her own words. Other cultural tools, such as paper and pen for drawings were also used. 


\subsection{Parent interview}

A semi-structured interview with parents took place after I had observed their child three or four times (Appendix 7). It was expected that the parents would not only provide me with new insights but also help me answer specific questions that had arisen from the field notes.

The interview followed a predetermined list of questions which I used to prompt myself to maintain continuity. My intention was to clarify thoughts derived from the field observations and generate new ideas about how to move on to gather further data. The participants and I conversed in Mandarin Chinese. During the process, I not only encouraged the parents to talk as much as possible, but also took opportunities to clarify any phenomenon that interested me. In doing so, I attempted to create coherent accounts with the parents. Dahlberg et al. (2001) claimed that interview accounts are a result of both researchers and informants.

During the data gathering phase, informal conversations were also made through which I sought feedback for any emerging enquiries. I took opportunities to have conversations with the parents and the children when they came to me or when I noticed emerging events or behaviours of the child that I needed to clarify for a further understanding.

\subsection{Teacher interview}

A semi-structured interview with teachers was conducted after I had observed a study child three or four times (Appendix 8). I considered teachers' perspectives of their teaching experiences and their insights of the study children to be valuable information for my research because they lived their daily lives with these children and they should be able to articulate their perceptions. In addition, since the study also aimed at identifying educational implications, teachers' current viewpoints were an important professional contribution for further consideration.

A schedule for the interview was followed along the lines of natural conversations. Similar to the parent interviews, I encouraged the teachers to talk about their experiences and insights concerning working with Chinese immigrant young children in general and the study child in particular. My role was mainly an active listener who 
occasionally sought clarification of any new insights generated during the interview process.

Beside the planned interviews, I was also alert to opportunities that might arise for informal conversations throughout the data gathering period. The teachers were encouraged to approach me with any information I might have missed. I talked to them at tea times to clarify emerging enquires or verify understandings.

\subsection{Ethical approval}

Before commencing the field research, approval was obtained from the Human Ethics Committee of Victoria University of Wellington. The study was premised on the ethical principle that participants were respected as people with human dignity, self esteem, and the right to privacy. My aim was that the participants would not be harmed or exploited.

Documents submitted to the ethics committee included a copy of an application form, information letters to the adult participants (Appendices 2 and 3), adult participant consent forms (Appendix 4), children's assent form (Appendix 5), and the data collection schedules (Appendices 6, 7 and 8). On the application form, I provided detailed information about the study, such as the research aims, questions, data collection methods, possible contributions of the study and potential harm to the participants. The information letters informed the participants about the study, my responsibility as a researcher in this research and how they could participate. The participants were also reassured about their rights including the right to withdraw from the study. The information letters and the consent forms for teachers were written in English and those for parents both in English and Chinese. The assent form for children included both pictures and words that children could understand and parents were requested to help me seek consent from children using the form provided. All the participants were asked to sign up the forms if they agreed to take part in the study. A consideration of the rights of the participations to "be heard, to participate, to have control of their lives" (Brooker, 2001, p.163), particularly those of the children's, was an important part of the ethical practice of this research. I believed that if the basis of the research laid in these people as essential knowledge-construction sources, I needed to respect them as I do myself. This thinking necessitated a flexible approach on my part that allowed my participants to conduct the interview in ways they wished, including 
choosing the time, setting, duration of the interview, as well as negotiations between us about its content.

As discussed above (see section 4.4.2), for all the participants, data gathering instruments included semi-structured interview schedules. Parents' interview schedules were created both in English and Chinese and those of the teachers were in English. The purpose of an English translation for the Chinese document was to help Englishspeaking people, particularly the ethics committee members and the participating teachers to understand them.

During the research process, I strictly followed the procedures as described in the ethical application. The participants were well informed of the study and of their rights when participating in this study. Even so, issues still arose which I reflect upon in a later section (see section 4.8).

\subsection{Data gathering procedures}

\subsubsection{First phase: The pilot study}

A pilot study was conducted to test the appropriateness of the research methods regarding the efficacy of my approach to the observations and interviews in drawing out Chinese children's experiences in New Zealand EC centres. I also aimed to identify what was feasible and should be focused on in the main study. This created an opportunity for reflection. I followed the procedure designed for the main study to take field notes and conduct interviews with parents, children and teachers. The proposed approach for data analysis in the main study was piloted too.

\subsubsection{Participants}

My pilot study involved two Chinese children: Kevin aged 4 years and 7 months and Tanya, aged 3 years and 6 months. These names are pseudonyms.

Kevin came to New Zealand from China when he was 10 months old and his parents were students at an educational institution in a major city in New Zealand. Kevin had a two-year-old brother who was taken care of by his grandmother at home. Kevin attended the centre five days a week on a full time basis and had been there for eight months. Prior to this, Kevin was at another EC centre for four months. 
Tanya was born in New Zealand and her parents had been settled in New Zealand for six years when they took part in the study. Tanya's mother worked at an office in a major city in New Zealand. Her father lived in China most of the time but visited New Zealand twice a year. Tanya's sister was 6 years old and studying at a primary school. Tanya started attending the centre eight hours a day and five days a week when she was three years of age. She had been at the centre for six months when I observed her.

\subsubsection{Field notes}

The time frame for child observations was not decided prior to the pilot study, as its purpose was to examine the feasibility of observations as a data collection technique for understanding the topic of this research. I allowed myself to carry on for as long as needed for this purpose. In the first three days, observations were conducted in the form of unstructured running records and there was new information flowing in. I noted down every detail about the child, the situations in which the child could be seen, the people and things the child interacted with, and the child's use of verbal and non-verbal languages. However, from the fourth day on, repeated scenarios emerged and on the fifth day, very few new episodes could be seen. At that time, I thought the study might be approaching the point of data saturation because little new information was being generated. Glaser and Strauss (1967) advise researchers that when similar instances occur repeatedly, the researcher is beginning to reach the point of data saturation. On the fourth day, my documentation changed from running records to a summary form of recording and I then only wrote in detail what appeared to be new or could be used to answer the questions I had formed as a result of the previous observations. In doing so, some particular scenarios received further attention, because of their value for understanding the child. When these occurred, I used a portable cam recorder to capture the actions. The cam recorder I used had to be switched on every five minutes and some precious moments were lost. For this reason, I decided to use a more advanced recorder for the main study to complete recording without disruptions. 


\subsubsection{Interviews with children, teachers and parents}

I was aware of the importance of having children understand me and having myself understand them during the interviews. Therefore, I spoke slowly and tried to be as clear as I could.

The interview with Kevin occurred on my last day of observation in his centre. With the help of one of his teachers, Kevin sat down with me. The teacher left the site as soon as Kevin and I started talking. I presented Kevin the beginning of the five stories. He appeared interested, but when I asked him to continue the stories, he did not seem to be keen to speak. The answer 'I don't know' characterised his response to my questions and I obtained very little information from him. The child was quiet and I noticed him examining my face. From his mother, teachers and my own observations of Kevin, I learned that he was a quiet child who took time to relate to people he did not know well. Although I approached him several times during the data gathering time, Kevin did not seek me for interactions and maintained a distance from me. Noting the tendency of children to give 'I don't know' answers, Brooker (2002, p.166) emphasised the importance of creating a "child-friendly" interviewing process. Even though I endeavoured to do so by allowing Kevin to be accompanied by a teacher and using a language he understood well, the child contributed little. I wondered if this was because we rarely interacted during the study. Kevin might have felt that he should not have talked much with me as I was not one of the regular adults in his centre.

This insight from my interactions with Kevin indicated the importance of strengthening relationships with the study children before expecting them to talk in an interview situation. With Tanya, I started to relax and tried to relate to her as much as possible. While I was in the centre, we played together on some occasions. My role was not simply as an observer but 'one of the teachers' according to Tanya. Very quickly, after the first day, Tanya looked out for me and always approached me with pictures and the work she had done. This seemed to work so that when we sat together, she was very willing to talk to me. Although she looked interested in the stories, I was surprised to discover that Tanya could also contribute little to the stories. She kept saying, 'you tell me'. Similarly to Kevin, Tanya also said 'I don't know' in many conversational turns. Tanya appeared to have difficulties constructing the stories. However, when I put the 
stories aside and asked her about her friends in the centre and her home life, she responded readily.

The interviews with the teachers of both children were conducted at the end of each study. The teachers were too busy to have time-out for interviews during work hours and both interviews took place at lunch time. In each case, the interviewees were supervisors of the teams. While I told the teachers that it would be helpful if they elaborated on their answers, much of the interview fell into a 'question and answer' session with me determining the topic of the conversation. The teachers were notably cautious of their answers and there were many hesitations and searching for appropriate responses.

The interview with the parents provided me with very detailed information from them. In contrast to the teachers' interview, the parents were relaxed and we talked much longer than planned. While the interview was similarly designed to be semi-structured the actual conversations with the parents were informal. Both parents were delighted to meet with me. They seemed to have also treated me as an expert for many questions because "I [the parent] know nearly nothing about my child's experience in the centre. It is great that I learn that from a person who knows early childhood education in New Zealand" (A parent interview). One parent openly requested that I check how the teachers worked with her child and to let her know whether it was a good place to stay. This placed me in an awkward ethical situation. I explained to her the non-judgemental purpose of the study and my need to keep information confidential. I also assured the parent that I would provide her with a summary of the findings at the end of the study.

\subsubsection{Pilot data analysis}

Data analysis in the pilot study consisted of two key stages: individual case analysis and cross-case analysis. Individual case analysis occurred alongside the data gathering. After each phase of data gathering, I typed out the data, uploaded images and saved them in the Microsoft word package under the name of each child. I then read the data repeatedly and extracted similarities and differences through drawing diagrams and tables to explore thinking, with reference to the research questions. The data were further processed and grouped, and then aligned with my research aims. Once all the data were gathered for each child, they were re-organised into themes and sequenced so 
that a holistic description for each child was developed. Upon completion of the data analysis for both children, I looked across the cases for recurring similarities and differences and created categories to identify their shared experiences.

\subsubsection{Insights from the pilot study}

The pilot study provided me with insights that led to a reshaping of my main study.

\subsection{Field notes}

The information gathered in five full days provided sufficient data for the analysis and I gained a clear picture of each child's experience. Therefore I decided that in the main study, the proposed observation period would be five full days.

Before the pilot study, I was very aware of the relationships I might develop with the study children for two reasons. One was that I was also Chinese, I looked similar to them and their family members. Another was that both children in the study had been informed by their parents that I would stay with them in their centres and watch them. In view of these factors, I perceived my presence to have an effect on my observations of children's natural behaviours. Therefore, for my observation of Kevin, I consciously kept away from him. For the whole study period, I approached him on very few occasions. Even so, I was not sure whether I was indeed minimizing my impact on his behaviours. Aubrey (2000, p.71) stated that "lack of contact... does not guarantee no effect of observer presence. Over time the familiar figure in the role of non-participant observer would be less likely to affect children's behaviour appreciably". This point alongside my reflection regarding interviewing Kevin, suggested that I should have allowed natural interactions to arise between myself and the children. This helped me gain richer information in the subsequent study with Tanya. For the main study, I decided to relate to children naturally, in the belief that an unforced relationship could facilitate the research process.

\subsection{Interviews}

I expected each child to relate the stories to his/her own life experience at an EC centre, but the actual interviews indicated to me that the children had trouble constructing the stories and relating the stories to themselves. As a result, for the main study interviews, I considered using the stories only as a starting point and conversed with the children in 
a natural way about what they wanted to say and to do. I would briefly tell the children the story, using the pictures to set the scene for the conversations and encourage the children to talk about their own experiences. Sumsion (2003, p.22) advises researchers to use the principles of "humility, reciprocity and community" to guide their interviews with children, to establish a productive research relationship with them and to eventually gain valuable information. In saying so, she emphasises that researchers should respect children, allow them to make decisions about the interview and interact with children as equals. My pilot study indicated that a strict focus on the stories did not work, because children did not have opportunities to make their own conversational choices.

Reflecting on my experience of interviewing Kevin, I realised it was useful to build a working relationship with children. This again highlighted the importance of interacting naturally with children so they might accept me as a friendly person with whom they wanted to talk.

Perhaps the reason for the teachers' caution in speaking with me during our interviews might be that they needed to consider their replies so that the centres and their practices could not be criticised. Although the participating teachers had read the information letters and signed the consent forms, they might still have felt responsible for protecting their centres and themselves. In view of this phenomenon, I decided to reassure my participants of the confidentiality of their replies during the interview.

In addition, because the teachers did not have the interview schedule beforehand, they seemed to have difficulty answering certain questions. In the main study, I decided to provide the interviewees with the schedules before the interviews so they might feel more prepared.

The question that arose during the parents' interview, with one parent asking me to judge the quality of their child's centre, indicated the necessity to start the interviews with clear ethical explanations to all participants. The parent's request highlighted the need for me to protect the research information from being disclosed across the participants. 


\subsubsection{Second phase: The main study}

\subsubsection{Participants}

Four boys and four girls participated in the main study. The parents of one child were from Taiwan and the other seven all had immigrated to New Zealand from mainland China. Among them, one boy, Jim, was 3 years and 1 month when the study started with him. The other three boys included Luke and Eden, both 3 years and 3 months, and Rick who was 4 years and 5 months. Amy was 3 years and 6 months, Sarah was 3 years and 8 months and Leah was 4 years and 6 months. Xiaohan was 4 years and 8 months and thus the oldest child. As in the pilot study, these names were pseudonyms. Details about each child can be found in table 5.1, in Chapter 5 .

\subsubsection{Field notes}

Before beginning my field notes, I was introduced to each child by either the parents or teachers. During the process, I did not hesitate to interact with the child when approached and allowed myself to initiate contact with them too. Although most of the time I stayed physically distant from the child I was observing, I tracked them closely, and played or talked with them whenever possible. I observed each child for one day a week over a period of five consecutive weeks. During the first three days of observations I documented, with paper and pen, the settings, the child's activities, the people they interacted with, and their languages and behaviours.

During the morning of the first visit to each child, I purposefully recorded in detail the child's experiences with peers during an hour-long free play session. From this, I aimed to gain initial information about each child's peer experiences in the centres from seeing with whom they tended to play, how they played together, and how long they played.

After two or three observations of the child a summary of the observational notes was shared with the child's teachers and parents during the interviews to inform the foci of the subsequent observations. They helped me construct meanings of certain points and advised me about how to move to the next steps. The last two days of the field work was recorded in a summary form. I simplified the writing but kept details of any emerging episodes or phenomena that the teachers and parents told me to pay attention to. I also noted in detail the child's behaviours that I thought would bring significant 
findings. When salient events arose, I videotaped the child's experience. The field notes and videotapes were reviewed, reflected upon and regularly cross-checked, so that the notes from previous sessions helped me to identify emerging themes for the subsequent sessions.

\subsubsection{Interviews}

The interviews all occurred after I had observed a child for two or three days. Parents and children's interviews were in Mandarin Chinese, while teacher interviews were in English. I had arranged to meet the parents before the interviews. I was a familiar and regular visitor for each study child and since I interacted with them, a certain rapport was established. The adult participants were provided with a written interview schedule and I sought their advice on the date, time and place for the interview.

Each child's interview began on the fourth day of data gathering, at free play time at the centre and lasted for about 30 minutes. I commonly began when finding the child doing something alone. The child was then asked to sit with me in a quiet place. After playing for a short while with the child, I would say, "Let me tell you a story about Hong (an imaginary Chinese child)". Following that, I told the child each of my stories and then asked: "I have finished my story. Hong watches TV before she sleeps. How about you then? What do you do at home?" I guided each child to move to his/her centre experiences by, for example, saying 'Hong goes to her centre with her dad. Who sends you to the centre? Why do you go to the centre?" My intention to learn about each child's perception of his/her experiences at home and in the centre was incorporated naturally in conversations with the child in line with the child's cues and responses.

Among all the children, Xiaohan, the oldest child, was the only one who spontaneously spoke at length about herself and her experiences without much help. The other children needed frequent prompts but they were able to give replies to my questions. When a child was speaking, I mainly smiled, listened and gave brief expressions such as 'oh'. The interviews were not audiotaped because I wanted these talks to appear as normal conversations for the children. Instead, I jotted down the child's key responses and wrote up the conversations immediately after the interviews. During the interviews, Luke, Xiaohan and Leah drew pictures. The other five children simply talked with me. 
Although both parents were invited, six of eight interviews involved only mothers of the children. Fathers of five children were not in the country at that time. One father had another event to attend. For the teacher interviews, at least one teacher in each centre participated on the basis that they knew the study child well. All the teachers and children were interviewed in the EC centres. Three parent interviews took place in cafés close to where they lived, three in their homes, one in a parent's work place, and one was at the EC centre. Each parent or teacher interview was about an hour long, and with their permission, they were audio-taped. I transcribed the data promptly after each interview and it was given to the participants for checking.

As planned, all the interviews followed a basic schedule of intended questions. The interviews began with the background information about the participant. For each parent, this was mainly about her/his family background of immigration and for each teacher about her teaching experience. What followed were conversations in which the parents or teachers told me about their experiences with the study child. I focused specifically on their expectations, strategies, challenges and needs. We talked about the child's experiences with peers and with adults, their learning of English and also their interests and needs. From these descriptions I asked further questions for clarification of their ideas, feelings and understandings. At times, I interjected with alternative explanations to encourage insights from a broader perspective, for example to say 'I wonder if it is also because she was...' By introducing alternative viewpoints, I adopted a dynamic sociocultural and phenomenological approach to cooperatively seek meanings with the participants about what had been expressed (Wells, 1993).

I searched for teachers' and parents' perspectives on working strategies for Chinese immigrant children. Interviews concluded with space for any comments. I then provided the participants with a summary of the field notes from previous observations of the child to clarify understandings and seek suggestions of possible foci in the subsequent field work.

The teachers and parents were approached again for informal conversations during the course of data gathering. Similarly, I repeated my ethical obligations when talking with them. In three EC centres, other teachers gave information about the study child. Informal conversations occurred with the parents at arrival and departure times and with 
the teachers at a time they nominated as suitable to talk. Purposeful interactions were initiated with each child too when I observed the child showing a particular behaviour that I wanted to learn more about. Not all the words from the informal conversations were recorded and only points considered useful for the study were recorded using paper and pen on the spot.

Table 4.2 shows the information about the data gathering methods for each child in the main study.

Table 4.2: Main study data gathering for each child

\begin{tabular}{|c|c|c|c|c|}
\hline & Child observation & Child interview & Teacher interview & Parent interview \\
\hline Frequency & Once a week & $\begin{array}{c}\text { Once } \\
\text { (semi-structured) } \\
\text { Ongoing } \\
\text { (unstructured) }\end{array}$ & $\begin{array}{c}\text { Once } \\
\text { ( semi-structured ) } \\
\text { Ongoing } \\
\text { ( unstructured ) }\end{array}$ & $\begin{array}{c}\text { Once } \\
\text { ( semi-structured ) } \\
\text { Ongoing } \\
\text { ( unstructured ) }\end{array}$ \\
\hline Duration & Full Day & $\begin{array}{c}\text { Half an hour } \\
\text { (semi-structured) }\end{array}$ & $\begin{array}{c}\text { An hour } \\
\text { ( semi-structured ) }\end{array}$ & $\begin{array}{c}\text { An hour } \\
\text { ( semi-structured ) }\end{array}$ \\
\hline Number & Five & $\begin{array}{c}\text { One } \\
\text { ( semi-structured ) } \\
\text { Many } \\
\text { ( unstructured ) }\end{array}$ & $\begin{array}{c}\text { One } \\
\text { ( semi-structured) } \\
\text { Many } \\
\text { ( unstructured ) }\end{array}$ & $\begin{array}{c}\text { One } \\
\text { ( semi-structured ) } \\
\text { Many } \\
\text { ( unstructured ) }\end{array}$ \\
\hline
\end{tabular}

\subsubsection{Main study data organization}

The search for significant concepts, guided by the research questions, began from the moment data gathering commenced and was ongoing throughout the fieldwork. During data gathering, I progressively narrowed the focus of the study, and some important aspects of the study emerged in the process. Once a field note was taken or an interview was completed, I read the notes or played the tape over and over again. I chose to look at them promptly while the experience of gathering these data was still fresh in my memory. Memos in my reflective journal about thoughts on these data were used to guide my subsequent research with each child.

Each case study resulted in two major sets of data, field notes and interview transcripts. A folder was created for a child in the Microsoft word system, which contained subfolders for field notes and interview transcripts, according to the dates they were taken. So, for each child, there was a total of five sets of field notes, three semi-formal interview transcripts (from the child, parents and the teachers) plus one document 
containing all the informal conversations about the child. Photographs and video clips for the child were stored in a sub-folder. All the data recorded in Chinese were translated into English.

\subsubsection{Data analysis}

Data analysis was undertaken for two purposes: (i) drawing together each individual child's experience to create a holistic description of the events within each case study; and (ii) synthesizing areas of congruence and differences across all the cases. Because the phenomenological approach had the potential to generate meaning from the firstperson perspectives, I drew upon its principles to gain meanings from the data as they were constructed by the people who presented them (Hycner, 1985). The informal conversations with the participants during the data gathering process and my reflective field notes were helpful here. Furthermore, given that human interactions and interrelated ways of interpreting data are an essential strategy of sociocultural research, developing joint accounts with my participants through involving them in informal conversations and in data verifications is built on sociocultural perspectives. The discovery of the meanings of these data was the result of social interactions and negotiations between the participants and me. The data analysis was inductive and descriptive. I aimed at describing and explaining Chinese immigrant children's experiences in a rigorous and detailed manner with a view to highlighting important insights.

\subsection{Individual case analysis and description}

1. The first step: gaining an initial sense of the whole

In line with the principles of phenomenological analysis (Giorgi, 1997), the initial work I did was to review the transcripts and the field notes to get a sense of the learning experience for each Chinese immigrant child and its meaning for them. I read and reread the field notes, listened to audio recordings of the interviews with the adults and watched videotapes repeatedly to gain sensitivity to the entire data. My reflective notes were not part of the case description and were not analysed, but they helped me to analyse the data and reminded me of how I understood something when it happened. 
2. The second step: coding topics

The second step of the data analysis entailed scrutinizing the data to develop preliminary codes for clustering around topics. It involved extracting the notes and transcripts that directly pertained to understanding a child's learning experience and putting aside the data that did not show close relevance to the research phenomenon. Although certain codes were developed during the preliminary step, coding topics was not a static process in my data analysis; later thoughts on including further items were also part of the progressive development of data. The work was carried out on a computer as illustrated in table 4.3 below, which illustrates the line-by-line coding of a field note extract of Xiaohan's behaviour.

\section{Table 4.3: A line-by-line coding of a field note extract}

\begin{tabular}{|c|c|}
\hline Field note extract & Coding \\
\hline Xiaohan calls out: "Rick" when seeing R walking into the room. & Initiate a peer contact \\
\hline $\begin{array}{l}\text { She goes to Rick and gets a paper blower from a box that Rick is holding. } \\
\text { Xiaohan blows it. }\end{array}$ & Following a peer \\
\hline $\begin{array}{l}\text { Red walks to Xiaohan, watching sideways. X does not pay attention to } \\
\text { Red. Xiaohan reaches her hand to Rick: "Rick ya. Zhe ge gei ni" [Rick, } \\
\text { this is for you], pretending to give the blower back to Rick. }\end{array}$ & $\begin{array}{l}\text { Ignoring a peer } \\
\text { Purposefully keeping a } \\
\text { peer }\end{array}$ \\
\hline $\begin{array}{l}\text { Xiaohan turns around and sees Ben: "Ben, kan. Wo hui chui" [Ben, look, I } \\
\text { can blow it], and blows again. }\end{array}$ & Initiate a peer contact \\
\hline
\end{tabular}

3. The third step: categorizing meaning units

Discovering meaning units was an important practice of data analysis, in line with the phenomenological approach (Hycner, 1985). It called for a close examination of the data and was achieved by studying the preliminary codes many times to see whether some of them illustrated a similar point. From the initial codes, I identified key words, phrases, sentences and extracts that indicated a child's experiences in similar ways and grouped them together. As I read and listened, I searched for patterns of meaning among all the initial codes. I looked across the field notes and transcripts to re-organise the segmented codes to establish links with my research questions. The approach employed at this step was to go beyond the data and to think creatively about it, and it required a continuous interaction with the data. As I read, I asked myself the question 'who, what, when, why, where and how?' In essence, this step constantly involved asking 'what is happening here?' and 'how does it help me understand the experience of this child?' As 
in the process described by Davidson and Tolich (2003), my understandings from the literature, insight into the children's learning context and each participant's ability to give meaning to the data all contributed to the categorizing step of the data analysis.

At times, I also encountered emerging incidents that could not be categorised into any existing categories. For example, when collecting and analysing Sarah's data, I noted her keeping a distance between herself and her peers when they were all sitting on a bench waiting for a turn to play. This behaviour had been unnoticed in the previous data gathered and did not fit into any existing analytical category. In that situation, I developed a new category of social withdrawal and paid attention to similar behaviours during the rest of my study with Sarah. This emerging category created an area of new interest and marked points for expansion that added a new dimension to my understanding of the research topic.

As in the second step, I worked with Microsoft Word to develop categories through reading notes, typing them, cutting and pasting key words, phrases and extracts.

The example shown in table 4.4 demonstrates the establishment of linkages among the initial codes from different data sets (child observations, interviews) to develop a category. 
Table 4.4: An example of organizing codes to develop a category

\begin{tabular}{l}
\hline \multicolumn{2}{c}{ Category } \\
\\
\\
\\
\\
\\
\\
\\
\\
\\
Forming a \\
bond \\
Chinese peers \\
for common \\
play and \\
mutual and \\
communication
\end{tabular}

Playing with Xiaohan is cycling outside. Lisa (an English girl) runs to her Chinese peers due on another bike. Lisa says: Xiaohan, chase me. Xiaohan to the common first gives her a glance, says nothing and carries on biking. 49 language seconds later, Xiaohan gets off the bike and walks inside (Field notes)

Karen: does she have a preference of friends?

Xiaohan's mother: I think she likes Chinese because they understand each other (Interview with Xiaohan's mother)

Jenny (Xiaohan's teacher): Xiaohan plays with the Chinese peers a lot. I guess because they understand each other Very occasionally, Xiaohan plays with English speaking peers (interview with Xiaohan's teachers)

Karen: who are your friends here?

Xiaohan: Rick, Ben, Peter (Chinese boys).

Karen: anyone else?

Xiaohan: maybe Red. I only play with Chinese children because we speak Chinese (interview with Xiaohan)

Xiaohan runs outside. She stands at the door and looks around. Xiaohan sees Rick, Ben and Peter (All Chinese) in the sandpit and runs to them. Xiaohan: "ni men zen ma dou zai zhe ya" [Why are you all here?] (Field notes)

'I don't play with little kid. They don't know how to play'.
Xiaohan sits at a table. Red, a 2-year-old Chinese girl goes to sit with her. Xiaohan sees her and quickly leaves. Xiaohan walks to the bathroom. Red waits for her outside. Xiaohan comes back and sees Red and says to her: "ni qu na bian wan. Buyao gen zhe wo" [You go there to play. Don't follow me], pointing to outside and then walks to the family area where Rick was (field notes).

Xiaohan's mother: Xiaohan is the only child in our family and she does not know how to play with little ones (mother interview).

4. The fourth step: thematizing

With further scrutiny of the data, it became evident that some categories could be linked and this led to the development of the themes. In Xiaohan's case, when putting together all the categories about her experiences with peers, the theme naturally became 'similarity-oriented peer relationships'. In recognition of the relevance of this theme to the second sub-question of the first research question, 'How do Chinese immigrant 
children experience their learning as a member of a group in New Zealand early childhood settings?', I then returned to the data to consider how this theme could be further developed to create an answer to that question that was as full as possible. As the data collection went on and new insights emerged, new codings were developed which were either grouped into an established category, or developed into new categories, and then into a theme.

Some examples of the themes developed at this stage of the individual case analysis are:

- interchanging language usage between English and Chinese;

- communicating in English with strategies;

- speaking Chinese when being alone;

- Chinese peers as a base of further peer relationships;

- treating teachers with respect;

- withdrawing social interactions; and

- cautiously responding to potential unfriendly peer treatments.

5. The fifth step: structuring the experience

Subsequent data analysis entailed a synthesis of all categories and themes into a coherent description of each child's learning experiences (see Appendix 10 for an example). The purpose of this step was to draw together the repeated key ideas to create an integrated account and explanation of each child's learning experience. Again, there was a focus on consistencies of meaning to reach understanding of the experience. All the codes, categories and themes were examined and reviewed again and connections were detected and created among relevant data. I stepped out of studying the parts and considered the whole picture and how each part contributed to it. This, according to Van Manen (1990), was a back and forth movement of data and the text created. Each individual case analysis was performed so that the final descriptions were specific to each child. This helped to keep intact the data for each case and to provide enough and appropriate evidence for the final cross-case analysis.

\subsection{Cross-case analysis}

After completing the individual case descriptions, I began a cross-case analysis to identify recurring patterns across the cases. This analysis went beyond individual case descriptions by looking across the cases so as to reveal their shared accounts. According 
to Miles and Huberman (1994), this is to see the commonalities and differences across many cases, thus develop more advanced descriptions and more vigorous explanations. I used the same process of cutting and pasting relevant narrative texts and created code headings as described in section 4.6.2.5.1. I reviewed all these children's cases periodically to search for patterns of meaning within and between the examples and codes, as well as for extracts that could suggest the shared and differing experiences. For example, the theme 'treating teachers with respect', as shown above, occurred for many children, so that I developed a cross-case file of data for this. Many of the themes and categories that had been developed in the individual case analyses were changed in this cross-case interpretation process for emergent common meanings across the data. At the same time, some data that were put aside in individual analysis were re-visited for new insights. The end result of the cross-case analysis created categories and themes that were meaningful for all the cases.

\subsection{Research rigour}

The criteria for rigour in qualitative research are trustworthiness and authenticity, which means that researchers should obtain the true voices of the participants and conduct the research in a way that a rich and accurate recording of the data is maximised (Toma, 2006). Silverman (2000) has a similar view, saying that the outcome of qualitative research relies on true dialogues between the researchers and the researched. Researchers need to "convince themselves (and their audience) that their 'finding' is genuinely based on critical investigation of all their data" (p.176).

To ensure quality measures were met for this study, I followed some specific procedures such as the approaches of participant validation and triangulation suggested by Bryman (2004). Participant validation means that researchers return to the research participants with their tentative results and refine them according to the feedback of the participants. There are three possible components of triangulation, consisting of (i) the use of a variety of data sources, (ii) multiple methods to investigate the issues or (iii) multiple theoretical perspectives to analyse the data (Bryman). Blaikie (1991) argues that a study may be more empirically valid and less biased if a range of perspectives is used and their individual strengths are capitalised. The purpose of this is viewed as creating a convergence in interpretation in the research, leading to perhaps "the interweaving of processes: discovery, telling, storying, representation" (Lincoln \& Guba, 2000, p.182). 
To validate findings, I returned a copy of the summary account of the observed phenomenon, and the interview transcripts to the adult participants to verify that the account was reasonable and reflected their knowledge and experience. Their feedback led to revisions and further points from them were incorporated into the data analysis. I requested parents to 'brief' the observation summary to each participating child for any possible modification of the account I had outlined. In view of my insider role, I was very cautious with this practice so as to avoid biases. The summaries for the parents and children were presented in Chinese. I made a follow-up request with each child after returning the child's copy to the parents to make sure that the child had indeed seen it and given opinions. To my delight, all the parents had done this.

I sought to triangulate the data through the use of several data gathering methods to gain insights into the research questions. The use of child observations, together with child, teacher, and parent interviews allowed a degree of cross checking of the data gathered. In addition to field notes and interviews with children, teachers and parents, I wrote research reflections in my journal to constantly scrutinise what I was doing and what I felt at the time. From these reflections, I was able to look for my preconceptions and work out how I might be able to minimise my impact. In the journal I also kept further thoughts on how to proceed with the rest of the field work and what contextual cues to look for. These thoughts helped identify salient scenarios for more attention. I also used this technique to record ideas about coding relationships to elaborate on the work of discovering themes and structuring case descriptions. Additionally, I used insights from two theoretical perspectives to analyze the data, that is, sociocultural and phenomenological perspectives. As noted earlier (see section 4.2), this combination of theoretical perspectives in the analysis of this study enabled me to gain information both from the children and the adults in their sociocultural contexts. The pilot study, which served as a testing technique for the data gathering procedures of my study, also provided scope for identifying issues that I needed to attend to in the main study, enabling me to refine the study questions. 


\subsection{Field work reflection ${ }^{4}$}

The key task of my data collection was to develop effective working relationships with my participants to obtain authentic and useful information from them.

The use of a phenomenological perspective required me to understand how my participants perceived their own experiences with the intention of accessing their lives. As the key instrument of data gathering, I needed sufficient information about Chinese immigrant children's experiences in New Zealand EC centres, and it was essential that I gathered the information in a way that was ethically sound and appropriate to the cultural needs of the participants. This led me to consider how to relate to my participants.

The data in this study were gathered from people of two major cultures. Given my Chinese upbringing and New Zealand tertiary background, I perceived myself to be reasonably knowledgeable about both cultures. However, this made me unsure of my ability to reconcile the ethical adherence with both cultures, because of the differences between them regarding how to approach people and deal with information. In their reflection on the challenges of research in intercultural situations, Salinas, Rance, Serrate and Castro (2000) coined the term "unethical ethics" (p.104), and questioned the adequacy of institutional ethical protocols for doing research in an intercultural context. They proposed that research needed to be ethically flexible in order to allow participants to negotiate with the researchers about how they would participate.

Salinas et al.'s (2000) term 'unethical ethnics has relevance to my research experience. I found it difficult to maintain a balance between being ethical and culturally sensitive because of the tension between my role as a Chinese immigrant, whom the parents could easily relate to, a researcher from a non-Chinese educational institution with a specific set of ethical rules to follow, and an EC lecturer, perceived by the participants to have "expertise in the chosen area of research" (A teacher interview). The following account illustrates some of the tensions I experienced.

\footnotetext{
${ }^{4}$ Parts of this section were first published by Guo (2009) in New Zealand Research in ECE 12.
} 
Firstly, the participating adults approached me for information and advice. Some teachers asked whether their practice was appropriate and they wanted to know how the Chinese parents perceived their practice. I also had parents ask what I thought of the work of teachers and of the quality of the childcare centres. In these intercultural contexts, the different languages and different cultural norms of communication created communication gaps between teachers and parents and it was not surprising that they expected me to help them understand each other. While pleased that the participants trusted me, I was also concerned about what information could be conveyed to them. I was clear that I could describe but could not judge, and I felt that it was not possible for me to satisfy the needs of one party at the expense of those of the other. Both parties' hunger for information indicated the value of this study but presented me with a dilemma regarding how to reply to them. Recognizing the importance of being ethical, I reiterated my requirement to protect all the people involved in the study. This approach worked for the teachers but not for all the parents.

Some parents told me that they were keen to know what I learnt about their child because they intended to provide assistance for their child. Thus, their intention when requesting information was not only to learn about their child but also to use the information to take further actions, which naturally enough, contributed a further dilemma for me in my response to them. The following was one of the notes in my research journal. It was written after I had verified the data with Eden and his mother. It is an example of how Eden's mother was doing more to assist Eden's learning.

I visited Eden's mother and Eden at their home with the summarised field notes and the interview transcripts that I have taken from them. His mother was warm as usual and we conversed in their lounge while Eden was playing around. In the summary of the field notes, I described Eden's learning behaviours in social situations. In response to his exclusive play with Chinese children, the mother immediately said that Eden needed to go to another centre with no or fewer Chinese children, so that he could learn English. In a rage, she called Eden in and blamed him for making no effort to play with English speaking children. Eden simply stood there with a confused and sad look. I was embarrassed as well as feeling guilty about what happened to poor Eden. Regardless of how I explained, the mother was adamant that Eden should be moved out of that centre. (Journal reflections, June, 2006)

This incident led me to reconsider the verification component of the field study. I had been mistaken to presume that the participants regarded all the described learning experiences as appropriate. The incident above illustrated that my understanding could 
have differed from that of the parents and some descriptions that I perceived to be positive could trigger possible negative reactions in the parents. Following this incident, I enclosed a note with the field notes that I shared with parents stating 'these data only document a specific period of a child's behaviours in his/her normal course of development; they do not provide sufficient insights into understanding the child and his/her learning setting'. Although parents' reactions to their child's behaviours still occurred, the note seemed to work. I did not see other parents condemn their children in front of me. Also, no further parents insisted on taking an immediate action after reading my notes, although I could not tell whether they did so at a later time.

Another issue I was aware of was the potential effect of my presence on the dynamics of the learning settings and thus the nature of the data. I felt that with the children knowing the purpose of my visit to them, my presence might have led some children to make a deliberate effort to capture my attention. I noted that Xiaohan, Leah, Luke and Jim tried to include me in their experiences. Some children, on the other hand, might have been concerned about their special status in the group because of my particular focus on them. On a few occasions, I noted Sarah giving me a quick glance when she was playing with peers or teachers. It is highly possible that some children changed their behaviours in my presence. In addition, my previous visits as an 'assessor' of student teachers' performance in some of the participating settings might have influenced the practice of the teachers there. In recognition of the likelihood of the effects of my presence, I endeavoured to minimise the impact by making no interventions and strictly following the centre practices.

Because children were the main participants in the study and they were being observed, the most important aspect of my data collection was to minimise the use of them as the objects of this academic gaze. It was important to remember that they were informed participants and therefore protected for being so (Morrow \& Richards, 2007). In view of Chinese parents' traditional role to be in charge of their children, as discussed in Chapter 3, I described to the participating parents why I was seeking their children's permission. Possibly because they had given their permission, the children did not appear to be uncomfortable with me around them. Furthermore, given the potential stress of my interviews for the children, I allowed each child to leave the interview place at any time and continued the interview when they were happy. Other children 
interested in our conversation were invited in, as I aimed to keep the study child in a natural learning setting where he/she was not seen as being singled out. Each child was only guided to contribute but was not coerced to give replies by any material incentives.

After the field study, in keeping with proper etiquette and as a validation tool, I contacted all the participants to check their interview transcripts. Following that, they were informed of the completion of my study and the possibility of meeting again for any points or issues that might arise in the subsequent phases of the study. They were all happy to talk to me again. This was a necessary step for me because I believe a research study involves team work and all the participants should be closely informed of its progress.

In contemplating social science research, Bryman (2004) admitted the difficulties of drawing a line between ethical and unethical practices. My overall data collection also told me that it is difficult to reconcile the ethical principles and cultural considerations in intercultural research. In this research experience, I saw myself constantly considering my role, the role of my participants, my expectations and those of my participants. The reality of ethics as applied in this research process is that I often needed to pause and reflect on tensions and dilemmas that emerged and to only respond after carefully deliberating on the implications of the various courses of action. I noted in the reflection journal as follows:

The relationship between myself and the Chinese immigrant parents generated extra demands on me, which was not predicted in the beginning of the research. It was difficult to relate to the parents in an ethical way.... I was fortunate that as 'an insider' [also a Chinese person], my hesitation to supply them with some information they asked for did not turn them away and most parents were enthusiastically taking part in the study regardless. I would, however, wonder how pure outsider researchers deal with such ethical issues. Would participants be less demanding to researchers of different cultures? Would 'outsider' researchers be in a more advantageous position for intercultural research because the interplay of cultures and the intercultural elements are less visible to them? (Journal reflections, January, 2007) 


\subsection{Chapter summary}

This chapter has described the methodological foundations of the study, encompassing the qualitative framework, the phenomenological position, and the approach of a multiple case study. It also detailed the information about the research instruments, participants, and the specific procedures for gathering data.

Data gathering in this study was conducted in two phases, the pilot and the main study phase. The pilot study was conducted to inform me of the feasibility of the research methodology planned for the main study. These data did not contribute to any analysis in the main study.

In the main study, data were gathered from observing and videotaping the study children, reflecting on the research process and interviewing the children, their parents and some of their teachers. Data were analysed in line with a phenomenological framework in two steps: individual case description that involved a search for individual meanings of the research phenomenon, and cross-case analysis, in which a holistic account was created that incorporated both individual and shared meanings of the research phenomenon. These steps saw the data firstly being written in the form of an individual case description and secondly as an account across all the cases. It was these data that provided the essential basis for investigating the two major research questions regarding understanding Chinese immigrant children's learning experiences in New Zealand EC centres, and identifying implications for supporting them.

During the field work, several issues received heightened attention, and the research experiences involved in responding to these issues were also reflected upon. The ethical dilemmas that I came across from working with the participants, especially with the Chinese immigrant parents provided critical points upon which to reflect on my experiences in this intercultural research. At the extremes of these experiences/dilemmas was Eden's mother's intention to move Eden out of the centre where this research was being conducted after finding out that Eden only played with Chinese peers there. While I made as many attempts as possible to protect the children from being harmed, what happened to Eden was very unfortunate and filled me with dismay that I had inadvertently caused him harm. There is a lesson to be learned from this incident: researchers need to be highly proactive in taking actions to uphold children's right to protection from harm, including from the power inherent in the 
parent-child relationship, or for that matter, in any adult-child relationship. In their review of New Zealand ethical guidelines for research with children, Powell and Smith (2006, p.127) wrote about "the power difference between adults and children" as a specific ethical issue to bear in mind when conducting research with children.

The following chapter, Chapter 5, positions the study children within their sociocultural contexts. It presents a summary introduction of the children, and then describes their perspectives of themselves and the perspectives of their parents and teachers regarding the children' experiences in ECE. 


\section{Chapter 5: Chinese immigrant families and New Zealand early childhood centres: The children in context}

\subsection{Introduction}

The aim of this chapter is to interpret the learning contexts of the study children within the sociocultural paradigm and phenomenological approach described in Chapters 2 and 4. To accomplish this, there is first a summary introduction to the children, followed by aspects of their learning experiences in homes and in EC centres, drawn from interview data from teachers, parents and the children. Relevant field notes also add to the picture of the children's learning contexts. They provide an answer to the second and third subquestions of the second research question which explores how Chinese immigrant children can be supported towards positive learning experiences in New Zealand EC centres. These two sub questions asked:

2.1 What are New Zealand early childhood teachers' expectations and practices about working with Chinese immigrant children and parents?

2.2 What are Chinese parents' beliefs and practices about childrearing and early education?

Through introducing the children, this chapter aims to provide a reference point in the thesis, where each child can be appreciated as a holistic individual, who is a member of two cultural communities.

In sociocultural perspectives, cultural beliefs, practices, expectations, tools, social interactions and cultural variations are all important influence on children's learning and development. Thus, children's learning experiences are immersed in a system of their own cultural beliefs, practices, expectations and choice of cultural tools, as well as those of the people with whom they have close interactions. This means that the learning experiences of the Chinese immigrant children should be contextualised by presenting information from the children themselves, their parents and their teachers. This chapter aims to achieve this goal and describe the sociocultural contexts which the children experienced. As explained in Chapter 2, sociocultural contexts are the influences from children's living environments that include both their families and their EC centres. 
Therefore the children's opinions as well as those of their parents and their EC teachers provide valuable insights into understanding the children's learning experiences in their sociocultural contexts.

I have a very clear purpose for obtaining parents' perspectives regarding the experiences of their children in families and their own experiences with their children. According to Rogoff et al. (2007), in settings where different cultures meet, learners develop a hybrid form of practice that interconnects their knowledge base generated from day-to-day functions in different cultural communities. This is also the point made by Wenger (1998) in his concept of 'boundary crossing', or 'expansive learning', that learning occurs in a changing combination of interconnected activity systems. Many factors inherent in family practices affect children's development in out-of-home learning settings (Cole \& Gajdamaschko, 2007; Rogoff, 2003). Gravelle (1996) notes, "Bilingual learners arrive in school, at whatever stage in their education, with a range of abilities and experiences to build on" (p.74). Given that children's learning experiences in their EC centres have features of their home experiences, it is important that I learn relevant information from the parents regarding how the children's cultural experiences in their families influence their learning and development in the centres.

A focus on people's opinions of their life experiences is central to the phenomenological framework which, according to Husserl (1931/1977), its founder, means that phenomenological researchers need to investigate and record people's experiences in the context of their own intentions. The phenomenological framework supports the attempt here to understand the research phenomenon using people's own voices.

The discussion in this chapter is also underpinned by the concept of 'learning communities' (Fleer, 2003; Hughes, 2003) involving “a relationship of 'working together and "working with" (Woods et al., 1999, p.206), and is "particularly appealing in the context of bilingual learners and their parents" (p.205). By building into the discussion the concept of learning communities, the present chapter addresses the children's learning and development as a collaborative endeavour (Hughes). This concept provides a backdrop against which an integrated picture of the experiences of 
the teachers, parents and children in the sociocultural community of the EC centres is developed.

This chapter will incorporate the notion of 'culture' by investigating how Chinese immigrant family culture simultaneously functioned alongside the mainstream culture of the New Zealand EC centres' teaching and learning programmes, or "the dominant Pākehā mainstream culture" (Ritchie, 2003, p.2), and how the children responded to the influences of the different cultures. I purposefully use the term 'Chinese immigrant family culture' but not 'Chinese culture' because of the transformation of original cultures in immigrant families (see section 3.1.2.1). Rogoff's (2003) notion of variation in cultural practice is used to identify the practices of the children, their parents and their teachers in their cultural communities. There will be a discussion of the findings, focusing on concepts including 'family', 'centre', 'multiculturalism' and 'cultural diversity'.

It is important to note here that the terms 'Kiwi' and 'Pākehā' will appear in various parts of the thesis, in line with how the participants used them, or with reference to Ritchie's (2003) discussion of mainstream culture of New Zealand ECE. The terms are interchangeably referred to as 'New Zealand European' (Callister, 2004). As a matter of fact, in their clarification of the term 'Kiwi', the participating parents acknowledged it as the mainstream European culture of New Zealand.

\subsection{Introducing the children}

This section presents background information about each study child. As shown in Chapter 4, the children in the study are referred to by pseudonyms, as Jim, Eden, Luke, Amy, Leah, Rick, Leah and Xiaohan. The background information was constructed from the description of each child provided by the parents and teachers and my own field note records. Table 5.1 presents key demographic and familial details about each child and their linguistic and peer resources within the EC centre. 


\begin{tabular}{|c|c|c|c|c|c|c|c|c|}
\hline Child & Jim & Eden & Luke & Amy & Sarah & Rick & Leah & Xiaohan \\
\hline Age & 3 yrs $1 \mathrm{mth}$ & 3 yrs 2 mths & 3 yrs 3 mths & 3 yrs 6 mths & 3 yrs 8 mths & 4 yrs $5 \mathrm{mths}$ & 4 yrs 6 mths & 4 yrs 8 mths \\
\hline Sex & Boy & Boy & Boy & Girl & Girl & Boy & Girl & Girl \\
\hline $\begin{array}{l}\text { Country of } \\
\text { Origin }\end{array}$ & Taiwan & China & China & China & China & China & China & China \\
\hline English level $^{1}$ & Minimal & Minimal & Minimal & $\begin{array}{l}\text { Routine } \\
\text { Conversations }\end{array}$ & $\begin{array}{l}\text { Basic } \\
\text { Conversations }\end{array}$ & $\begin{array}{l}\text { Almost } \\
\text { competent }\end{array}$ & Competent & Minimal \\
\hline Chinese level & Competent & Competent & Competent & Competent & Competent & Competent & Competent & Competent \\
\hline $\begin{array}{l}\text { Chinese peers } \\
\text { available in the } \\
\text { centre }\end{array}$ & Leah & Jo \& Others & None & Kevin & None & $\begin{array}{l}\text { Xiaohan, Peter, } \\
\text { Ben \& others }\end{array}$ & $\begin{array}{l}\text { Jim, Fang \& } \\
\text { others }\end{array}$ & $\begin{array}{l}\text { Rick, Peter, } \\
\text { Ben, Red \& } \\
\text { others }\end{array}$ \\
\hline $\begin{array}{l}\text { Regular play } \\
\text { peers in the } \\
\text { centre }\end{array}$ & ${ }^{2}$ Leah (Chinese) & Jo (Chinese) & None & $\begin{array}{l}\text { Kevin } \\
\text { (Chinese) }\end{array}$ & None & $\begin{array}{l}\text { Xiaohan, Peter, } \\
\text { Ben (Chinese) \& } \\
\text { Allan (English) }\end{array}$ & $\begin{array}{l}\text { Jim, Fang } \\
\text { (Chinese) \& Mary } \\
\text { (English) }\end{array}$ & $\begin{array}{l}\text { Rick, Peter, } \\
\text { Ben \& Red } \\
\text { (Chinese) }\end{array}$ \\
\hline $\begin{array}{l}\text { Childcare } \\
\text { attendance }\end{array}$ & 1 month & 3 months & 2 months & 4 months & 10 months & 2 yrs $5 \mathrm{mths}$ & 2 yrs $4 \mathrm{mths}$ & 2 months \\
\hline $\begin{array}{l}\text { Previous } \\
\text { childcare } \\
\text { experiences }\end{array}$ & $\begin{array}{l}2 \text { wks in NZ } \\
\text { (New Zealand) }\end{array}$ & $3 \mathrm{mths}$ in NZ & None & $\begin{array}{l}1 \text { yr } 6 \text { mths in } \\
\text { China }\end{array}$ & None & None & None & 2 yrs in China \\
\hline $\begin{array}{l}{ }^{3} \text { Teachers who } \\
\text { participated in } \\
\text { the interviews }\end{array}$ & $\begin{array}{l}\text { Carol, Helen, } \\
\text { Ellie (Chinese) }\end{array}$ & $\begin{array}{l}\text { Sue, Dawn } \\
\text { (Chinese) }\end{array}$ & Nicole & Mary, Iby & $\begin{array}{l}\text { Gail, Casey, } \\
\text { Natalie }\end{array}$ & $\begin{array}{l}\text { Jenny, Iris, May } \\
\text { (Chinese) }\end{array}$ & Carol, Julie & $\begin{array}{l}\text { Jenny, Chris, } \\
\text { Fran, May } \\
\text { (Chinese) }\end{array}$ \\
\hline $\begin{array}{l}\text { Usual family } \\
\text { structure }\end{array}$ & $\begin{array}{l}\text { Mother, older } \\
\text { brothers (Father } \\
\text { in Taiwan) }\end{array}$ & Mother, Father & $\begin{array}{l}\text { Mother, Father, } \\
\text { Younger Sister }\end{array}$ & $\begin{array}{l}\text { Mother, Father, } \\
\text { Older Sister }\end{array}$ & $\begin{array}{l}\text { Mother, Father, } \\
\text { Older Sister }\end{array}$ & $\begin{array}{l}\text { Mother, Younger } \\
\text { brother (Father } \\
\text { in China) }\end{array}$ & $\begin{array}{l}\text { Mother, Older } \\
\text { sister, Grandma } \\
\text { (Father in China) }\end{array}$ & $\begin{array}{l}\text { Mother (Father } \\
\text { in Australia) }\end{array}$ \\
\hline
\end{tabular}

1 Data sources for compiling the children's language 'levels' including parents' and techers' informal assessment recorded during intervies, supported by my observations of children's langaguage used in the ECE centres. 'Minimal' means that the children are able to cope with very few communication contexts in English. 'Basic conversaton' refers to the chileren's abiality to comprehend and respond to some routine-related communication tasks in English. When children are able to comperehend and respond to nearly all routine activities in English, their English is concered to readh the level of 'Routine conversations'. Being 'almost competent' requires chiodren to manage many daily conversatons in English. 'Competence' is demonstrated when children are ableto deal with all elarning experiences that require the use of a language/s.

2 Leah and Jim were in the same EC centres and so were Xiaohan and Rick.

3 Due to the fact that the teachers were interviewed at different times for each child, for some children like Leah and Jim, as well as for Xiaohan and Rick who were in tehsame EC centres, the teachers who participated in the interviews were not exactly the same. Despite this, all the Chinsee-speaking techers in the EC centres participated in the interviews. 


\subsection{New Zealand EC teachers' beliefs and practices about working with Chinese immigrant children}

\subsubsection{Attending to the children}

The teachers all stated that their programmes were 'child-centred' and that this meant that they based their work with Chinese immigrant children on their needs, interests and individual experiences:
Iby:
Our programme is child-centred and we focus on the [Chinese immigrant] children to help them settle.
Julie \& Carol:
We are trying to help these children discover their interests.
Nicole:
We pay attention to Luke and talk a lot with him.
Sue:
We pay attention to Eden and make sure that he is happy.
Gail:
We give Sarah individual time and attention and focus on what she wants. We take the view that we should focus on the child.
Jenny:
The teachers here are consistent on the point that we focus on the Chinese immigrant children. We observe them closely to find out what they like and dislike.

The field notes also provided evidence of the teachers' attention to the study children. Having observed the teachers in their centres, I was able to note instances of their childcentred practices. For example, teachers maintained interactions with the children by listening and eliciting contributions from them. The teachers all adopted a particular speaking approach, such as talking slowly, employing examples and using appropriate body language to communicate with the children who had not yet developed competence with the English language. Teachers also kept a learning record for each study child that included examples of the child's learning experiences and teachers' notes. All the teachers implied in our interviews that they observed the children both informally and formally on an ongoing basis to uncover the children's interests and needs.

Teachers' attention to the study children was also noted when they attempted to focus on the children's particular strengths, which for many teachers seemed to be the children's motor skills and their ability to complete tasks (teacher interviews). On some occasions, I recorded the teachers calling to a study child to join an activity that required 
hand co-ordination skills. Often, the child was allowed to take time to work it out and the teachers' words of encouragement in those situations were often noticeable. The purpose of this for them, according to Sarah's teacher was to make Sarah proud. Leah's teachers made a similar statement that: "we are letting these [Chinese] children know that they are great".

Another skill that the teachers paid attention to for some of the children was the children's bilingual abilities. I observed teachers ask Amy and Leah to help them communicate with other Chinese children. Leah's teacher Julie said that "we want Leah to know that it's good to know two languages".

Many teachers stated in our interviews that attending to children was a practice grounded in the child-centred teaching philosophy of their centres. In New Zealand ECE, this has a professional orientation (Broström, 2003; Gunn, 2000) and according to Corrie (1995, p.4) it is "a deeply rooted implicit pedagogical knowledge". Thus the teachers' practice here demonstrates their absorption into a working tradition. As a result, the teachers believed that holding a child at the centre of their attention enabled them to work effectively with that child.

In the analysis of the children's learning experiences I began to get a sense of the importance of the child-centred tradition within Te Whäriki and how this guides EC teaching practices (Cullen, 1996; Doddington \& Hilton, 2007; Wu, 2009).

According to Burman (1994), the child-centred teaching philosophy is also an approach that teachers and schools use to separate children from their social relationships, particularly those with their families. In this philosophy, the goals are couched in terms of what children do in their schools or preschools. For this reason, the implementation of a child-centred approach could lead teachers to work with children in the absence of the children's families. While my study did not find evidence that indicated teachers' purposeful actions to separate the Chinese immigrant children from their families, the data did give reasons to believe that teachers' underdeveloped relationships with Chinese immigrant parents could indeed have an important bearing on their focused practice with the children. During the field research, I noticed very few examples of the teachers communicating with Chinese parents, and confirming this, all the teachers also 
described their relationships with the Chinese immigrant parents as a weak area in their teaching practice.

Mary: We do not know much about these [Chinese] children's families.

Jenny: $\quad$ We haven't done much work with the Chinese parents.

Sue: $\quad$ We should learn more about the Chinese children from their families.

Nicole: $\quad$ We know very little about Luke's family.

Iris: $\quad$ We'd love to cooperate with the Chinese families but for some reason have not done well.

Natalie: We hardly talk with Sarah's parents.

On exploring the issue with the teachers, several reasons for their underdeveloped relationships with Chinese parents emerged: the parents were too busy; the parents were hard to understand; the parents did not want to share information with them; they did not know how to interact in a culturally appropriate way with the Chinese parents; there was little useful insight that the teachers could gain from the parents; and working with the children was not difficult and therefore the teachers needed little help from the parents. Some teachers also reported feeling nervous when parents were hovering around.

Many teachers indicated that Chinese immigrant parents appeared to be too rushed to engage in communication. For example, Sarah's teachers said "Sarah's parents are rarely involved in the centre's programme because they are busy". Similarly, all the other teachers mentioned that they hardly met the Chinese immigrant parents. This was a phenomenon in the observational data as well. Leah's mother was the only parent who stayed in the centre between five and twelve minutes in the mornings when I was observing Leah. The other parents all rushed in and out, and typical interactions between English speaking teachers and Chinese parents were smiles. The following notes were recorded during morning arrivals of all the participating parents on my first visit to their child. 


\section{Table 5.2: Parents' morning arrivals with the children}

Jim's mother brings Jim in, puts Jim's bag in his locker, gives Jim a kiss on the cheek and walks out. When she turns back to see Jim, the mother meets the eyes of Jim's teacher Helen and waves 'goodbye' to her with a smile. Helen says: "bye" to her.

Amy's father brings Amy to the entrance and stops at the recording book, writing down the time of Amy's arrival. Amy walks into the room by herself. Her father waves 'goodbye' over the door. Amy: "zai jian" [bye.] On his way out, Amy's father meets the centre manager and smiles to her. The manager smiles back.

Leah walks in the door. Her mother is following. Leah puts her bag in the locker and walks to the room where the children and her teacher Ellie are talking. Leah's mother stays in the main play room. She then goes to the notice board, watching, and then goes to watch Leah from the window. Carol (Leah's teacher) sees her: "not busy today?" Leah's mother: "no, but I am leaving soon". Carol walks away from her. The mother watches Leah for a while and leaves.

Xiaohan's father comes in with her. He places Xiaohan's bag on a shelf and follows Xiaohan to her teacher May. May: "Hi, Xiaohan". Xiaohan's father: "wo zou le" [I am leaving.] Xiaohan: "zao dian lai jie wo ya" [come to pick me up early.] Xiaohan's father nods and walks out.

Rick's mother stops at the entrance: "jin qu ba" [walk in.] Rick gives his mother a cuddle and walks in. The mother watches him walking into the room and leaves.

Eden's mother brings him in and hands the bag to him: "mama 5 dian lai jie ni" [mum comes at 5 o'clock.] "Hao hao che fan, ting lao shi ha" [eat well and listen to the teachers.] Eden: "mama zai jian" [mum bye] and walks in. Eden's teacher Cathy is by her side, talking to another parent. She sees Eden's mother and gives her a smile. Eden's mother (to Cathy): "morning” and walks out.

Luke's father comes with him, holding his bag. The father puts the bag away and then takes Luke's hand and leads him to the toilet: "zou, shang ce suo" [let's go toilet first.] When they come out of the toilet, the father bends down towards Luke: "bu yao wang le ding shi qu ce suo ya" [don't forget to go to the toilet regularly] Luke: "hao de" [ok.] The father: "na zai jian" [bye then.] Jess, a teacher is playing with two children at a table by the door and sees Luke's father leaving and says "bye" to him. The father: "bye", walking out.

Sarah's father leaves the car and takes Sarah out. The father: "baba bu jin qu le. Mama hui lai jie ni de. Zai jian" [dad is not going in with you. mum is picking you up later today. Bye for now.] Sarah: "bye", and walks in. The father gets in the car and leaves.

So, during these arrival times, the parents and teachers did not communicate at all about the children. They either did not see each other; interacted with a smile, a single word 'bye' or 'hi' or with a few neutral words.

Luke's teacher Nicole described Luke's father's limited English as a barrier for her to communicate with him, therefore, "I do not talk to him". Other teachers mentioned their inability to speak Chinese, and the Chinese parents' lack of English made their communications difficult. 
Some teachers thought that they learned little about the children from their communication with the Chinese immigrant parents because usually teachers gave information and the parents listened. Sarah's teacher, Gail, told me "when we talked to Sarah's parents, they just listened". Xiaohan and Rick's teacher, Chris, had a perception that the parents did not want to express an opinion: "we show the parents the children's work and learning stories. The parents love seeing that but they don't comment". For Leah and Sarah's teachers the parents did not want to interfere. Leah's teacher Carol said: "Leah's mother has embraced our philosophies and practices. She seems happy with what we do". In much the same way, Sarah's teacher Gail said: "Sarah's parents trust us. Sarah's sister was here and we know each other well". While the teachers gave varied reasons for why the Chinese parents were reserved with their input, they seemed to have agreed that these parents did not reciprocate teachers' attempts to communicate.

Another reason offered by Dawn, Eden's Chinese-speaking teacher, about a lack of communication with the Chinese immigrant parents was a perception that it was unlikely teachers would gain useful information from these parents. Dawn told me, “Chinese parents do not talk about children's learning and when given opportunities, they would just make requests about what the children ate at the centre, or how they got a bruise for example". For Dawn, "because Chinese parents focus on different things from teachers, conversations with parents are not very useful". This led her to think that "unless there is a real need, it may not be necessary to communicate with the Chinese parents".

As a Chinese-speaking teacher, Dawn was here able to draw from her two cultural experiences to identify the difference between the Chinese parents' focus and that of New Zealand EC teachers. In comparison to the Pākehā's teachers' opinions, Dawn offered an explanation which she illustrated with useful evidence from her conversations with Chinese parents (see also section 5.3.4).

Some teachers attributed their underdeveloped relationship with Chinese immigrant parents to their lack of confidence in appropriately communicating with the parents (Amy's teachers) or a desire "not to offend them" (Eden's teacher Sue), due to the teachers' limited knowledge about the Chinese culture. 
Several teachers, including those of Sarah, Amy, Jim, Leah and Eden told me that they had learned that when some Chinese immigrant parents were hovering around, the children were behaving badly. For these teachers, too much involvement of these parents could have a negative impact on their work with the children.

Mary: $\quad$ The children have no problems but their parents' over anxiety and apprehension make the children unhappy and unsettled.

Although some teachers identified specific challenging behaviours of Chinese immigrant children, including putting on layers of layers of clothes (Sarah and Amy's teachers); snatching things (Luke and Eden's teachers); watching instead of engaging in learning activities (Eden's teacher), and crying excessively and not being able to be calmed down (Leah and Jim's teachers), the teachers were all positive that their work with Chinese immigrant children was not difficult because "these children try" (Xiaohan and Rick's teacher Jenny) and "they will gradually move in" (teachers of Amy, Sarah, Luke, Eden, Leah and Jim). In addition, all the teachers pointed out that the children respected teachers. This, according to Jenny, made teachers' work with them easy.

In her study of teachers from mainstream cultures working with diverse cultural children, Keats (1997, p.56) reported that "many [professionals] prefer to be able to deal only with the child, finding the parent from another culture difficult to understand". While this study supports this statement, the teachers' opinions were that it is more than just difficulties in understanding Chinese immigrant parents that led them to prefer to work with the children. The range of teachers' beliefs and practices about their work with Chinese immigrant parents, thus, offers scope for interpretation of relationships between people of different cultural communities. The finding that the teachers had clear intentions to support the children through focusing only on the children constitutes a useful empirical basis from which to investigate the complex relationship between New Zealand EC teachers and Chinese immigrant parents.

When Daniels (2001) spoke of a learning community as a site for regular interpersonal negotiation and communication, he suggested the need to focus on people's attitudes and actions. From the examples above, it is clear that a learning community was not established between Chinese immigrant parents and the New Zealand EC teachers 
because they neither demonstrated the necessary attitudes towards relating to each other, nor took actions to relate to each other. Given that family members are a primary contributor to children's learning and development (Rogoff, 2003), the finding that there were limited interactions between Chinese immigrant parents and New Zealand EC teachers, must be taken into account when seeking to understand the children's learning experiences. It also raises implications for teachers to find ways to build a learning community with these parents to better facilitate learning and development for the children in their EC centres.

\subsubsection{Treating children 'all the same'}

The teachers made a number of statements which suggested that they believed that Chinese immigrant children were no different to children from the mainstream culture and that any difficulties they might experience were typical of children who were settling into a new environment.

Mary: $\quad$ I could not see any behaviours of Amy that would suggest Amy was different from Kiwi children.

Sue: $\quad$ Same as other children, Eden looks happy.

Carol: $\quad$ There is nothing about Leah that we need to be concerned about. Her behaviours are strong at times but it is typical of a young child.

Nicole: Luke is in transition. He will fit into the group. It is not a good idea to single him out for his differences.

Helen: We are trying to include Jim into the group in the same way as we do with every other child.

Gail: $\quad$ Sarah and the other Chinese children we had are no different from local children. They only need more of our time and encouragement.

Fran: $\quad$ Because she is not familiar with the new environment and she cannot speak English, Xiaohan has difficulties. She is getting better and this is the same as all the children.

These beliefs seem to have led the teachers to decide that the children should be treated the same as their non-Chinese peers. Sue articulated this as "we promote a practice that immigrant children are accepted and treated in the same way as 'Kiwi' kids"'.

As well as the belief that the children were similar to their local peers, some teachers recognised that these children were sensitive and apprehensive. Helen said, "Jim looks 
very sensitive and I think emphasizing his difference might make him unhappy". Sarah's teacher Casey gave the same opinion: "Chinese immigrant children are very sensitive and I don't think it is a good idea that we focus on their differences".

I was interested to find that of all the replies from the teachers about the equal treatment they gave to children, none came from the four Chinese-speaking teachers, perhaps because the latter may have been more able to recognise the children's differences. For example, my informal conversations with the Chinese-speaking teacher Ellie suggested that she was aware of some differences between Chinese children and children from non-Chinese cultural groups, but she did not address them as an issue because "children change everyday. It is not really needed to emphasise their differences". May, another Chinese-speaking teacher, noted: "Yes, Chinese are different from Kiwis. Like myself, I am different from our Kiwi colleagues, but given the same routines and the same programmes, these differences should give way to what we do together as one group". These two Chinese teachers' statements suggest the Chinese-speaking teachers, regardless of their ability to identify differences for Chinese children, did not want to make them visible, and they were willing to share with their Pākehā colleagues the same teaching approach.

The teachers' words were consistent with their practice because I observed few examples that illustrated any explicit attempts by the teachers to make visible the study children's differences. The following conversations between Luke's teacher Nicole and two children showed that Nicole did not want to emphasise the difference between Luke and his peers.

\footnotetext{
A boy: "I don't like Luke. He can't talk". Another boy: "he is Chinese (voice up)". Nicole: "Luke is learning English and he will speak". The second boy again: "but he is Chinese, right?"

Nicole: "he is Chinese, but he is learning English". (Luke/ $3^{\text {rd }}$ visit, $3: 25 \mathrm{pm}$ )
}

Nicole used the word 'Chinese' given by the boy but did not elaborate on it. Instead, she shifted her response to indicate the similarity between Luke and his peers, thus trying to put aside Luke's difference. 
In addition, there was strong evidence across the cases that the teachers included the children in all aspects of their programmes. Therefore it is reasonable to infer that they did not intend to differentiate these children from other children.

The teachers' practice of treating the children similarly to all the other children was also identified in Chan's study (2006). When reporting EC teachers' beliefs in working with diverse cultures, Chan wrote, "some early childhood teachers believe that they do not differentiate in their treatment of children because all children are the same" (p.34).

Another of the teachers' principles regarding implementing the 'same' teaching programme could be a belief that "equipping children with the skills and abilities they need to succeed in mainstream schools is the best outcome for any child, regardless of cultural or ethnic differences" (Brewer, 2007, p.73). Although the teachers in this research did not articulate that the dominant Pākehā mainstream pedagogy was the most appropriate for the children, their actual practice indicated that the teachers regarded the study children as having the same needs as children of mainstream cultural groups. Therefore underpinning the teachers' work was a view that mainstream pedagogy was a suitable means for these children.

In a very recent study of New Zealand EC teachers' perspectives of their teaching strategies, Alvestad, Duncan and Berge (2009) reported that: "the teachers described to us ...traditional teaching approaches to children's activities and learning" (p. 10). If the EC tradition in New Zealand was grounded in the "white, European culture" (Terreni \& McCallum, 2003, p.7), or "the dominant Pākehā mainstream culture" (Ritchie, 2003, p.3) it is not surprising that the teachers in my study also promoted it in their practice.

Nonetheless, the 'same' teaching approach that the teachers adopted here is in direct opposition to the practice of 'diversity' (Dijkstra et al., 2001). In one sense, 'focusing on children' and 'treating children all the same' are both fundamental markers of monoculturalism because they tend to separate children from their cultural background and to shape children's learning experiences in a universalised way (Burman, 1994). Therefore, an interpretation of the data is that the teachers were not implementing the approach of cultural diversity. This finding supports the conclusion of previous researchers, namely de Melendez and Beck (2007), Ogbu (1992), Rhedding-Jones 
(2005), and Ryan (1999) that responses to cultural diversity encounter difficulties and resistance in the education sector. This also confirms one of Cullen's assumptions (1996) that many EC educators in New Zealand are prepared to live with the traditional approach of teaching practice instead of making curriculum innovations.

It can be seen from this study that regardless of a culturally diverse EC learning environment being promoted in Te Whäriki and other EC policy documents, these policy initiatives had not led to engagement with different cultures in the centres involved in my study. Rather, what seemed to have occurred was that the teachers lived in a self-constructed learning community, in which there were disparities between what they did and what they were expected to do.

One can argue that the 'same' teaching approach reflects, at least in part, teachers' beliefs in "a naturalised, individualised model of childhood" which underpins the approaches of child-centred education (Burman, 1994, p.163). This is an important point, as these beliefs shaped the teachers' practice, and thus the learning experiences of the study children. For example, such beliefs can lead teachers to interpret cultural behaviours of the Chinese immigrant children as natural and neutral. Given that immigrant children came to EC centres with culturally-oriented behaviours (Igoa, 1995; Rogoff, 2003), treating them in the same way would have constrained the teachers from helping the children in culturally appropriate ways. The 'same' teaching approach and the child-centred practice are also significant challenges to the Vygotskian notion that teachers should work in children's zone of proximal development (Vygotsky, 1978). The 'same' teaching approach could result in the possibility that children's potentials and their ability to move further are unrecognised. The interviews with the Chinese immigrant parents showed that, at the very least, treating the children in the same way as all other children, whether knowingly or not, did not meet the culturally-based educational expectations of the parents.

\subsubsection{Bringing in Chinese culture}

Many teachers in this study described how they encouraged the Chinese immigrant children to feel at home and how the children's family culture was valued in their practice. This included encouraging the children to speak their first language; allocating children who were Chinese speakers to support the study children who had recently 
started at the centres; using resources that were relevant to the children; and having professional training sessions to develop teachers' knowledge about Chinese culture.

All the teachers claimed that it was important that they allowed and encouraged the children to use their first language. In this way, according to Rick and Xiaohan's teacher Jenny, "the children feel at home". Jim and Leah's teachers said: "this keeps their culture". Eden's teacher Sue stated "Chinese children can use their own language to seek help from whoever they are comfortable with; therefore the environment is not threatening to them".

Chinese peer support was identified as an explicit practice to support the children. Leah and Jim's teacher Carol said that "we asked Leah to help Jim". Amy's teachers also pointed out their reliance on Amy to help Ken. While Eden's teachers did not make peer support a visible teaching strategy, they said "because of the large proportion of Chinese children, a Chinese child could easily find support when the child first started and we are happy for them to play with each other". Rick and Xiaohan's teachers made a similar point and said that "we are amazed at how quickly a Chinese child could connect with Chinese peers. It helps them all".

The teachers from the centres where there were Chinese teachers pointed out the benefit of having these people around them to give special support to the children.

Jenny: $\quad$ May makes our work with the Chinese children so easy because the children relate to her naturally.

Carol: $\quad$ Ellie is an asset to us. She understands Chinese children and their parents and is able to give them a sense of security.

Sue: $\quad$ The Chinese children approach Dawn a lot and the parents want her too. Dawn provides some security to them.

The teachers from two centres mentioned that they incorporated Chinese cultural tools in the centre programme. These include, as Rick and Xiaohan's, and Leah and Jim's teachers indicated, family photos, Chinese words displayed in the room, Chinese food for tea, basic Chinese words used by teachers and celebration of the Chinese New Year. Jenny elaborated: "we displayed the room in the Chinese way on their New Year's day, 
by putting on lots of red things and some big Chinese words. We asked the Chinese parents to make dumplings for us that day".

In recognition of their need to develop competence in other cultures Sarah's teachers said that "we had professional training sessions on Chinese culture and Chinese ways of raising children and we learnt a bit of Chinese too". Gail, the manager of this centre was once observed to say: "gao xing ma?" [Are you happy?] to Sarah.

The incorporation of Chinese cultural tools was observed in a few centres during my field work. In Xiaohan and Rick's centre, their teacher Iris asked May to show children the Chinese version of Twinkle twinkle little star. Amy's teacher Iby learnt 'good morning' in Chinese and used it with Amy and her Chinese peer Ken. Chinese greeting words were on display in Jim, Leah and Xiaohan and Rick's centres alongside the greetings in other languages.

It appears that the English-speaking teachers in this study relied on the Chinese language and people, as two available resources to work with the children. However, in most cases, the use of Chinese tools was as described by Rhedding-Jones to be "in a tokenistic manner" (2005, p.131) or an "add-on" (p.134) to be only seen (photos, written words), sometimes heard (songs, verbal words), or occasionally celebrated (festival), but not embedded in the daily programmes of the children. This practice also reflects what Banks (1988) has referred to as superficial multicultural practice characterised by a limited inclusion of ethnic content in the curriculum on the condition that "the mainstream curriculum remains unchanged in terms of its basic structure, goals, and salient characteristics" (p.37).

These difficulties are also reminiscent of Cullen's argument (2003) that lack of competence in identifying diversity, variation in teachers' knowledge base, and the contexts in which teachers work all limit teachers' effectiveness in implementing practices that respect cultural diversity.

In addition, it is important to note that the teachers all used 'Chinese culture' in their discussions of the study children's family culture. None of them specifically referred to the children's family culture as 'Chinese immigrant family culture'. This is a very 
significant finding in this research. When associating 'Chinese culture' but not the culture of Chinese immigrant families with the children, it is very likely that the teachers regarded the children's family culture to be the same as that of all the Chinese children they worked with, including possibly of the Chinese people they had known as neighbours or from books. The teachers' generalization of diversity is not a unique finding to this study. Bennett (2006), Rhedding-Jones (2005), and Siraj-Blatchford (2001) have all noted that the generalization of cultures has been a noticeable practice of multicultural education.

Banks (2006) conceptualises school as a microcultural system that constitutes many norms, values, roles, statuses, and goals, and that student and teacher cultures are too diverse to be integrated into a school culture. Regarding cultural integration, considerable work may go into considering such issues as what differences are worth reconciling and the ways in which they can be reconciled. In relation to this research, the inclusion of only accessible and celebratory tools of Chinese culture and the teachers' generalization of the children's family culture could be attributed to the complexity of culture, complexity of diversities and difficulties in relating to other cultures.

Furthermore, because the teachers valued a child-focused approach, they might have formed a belief that although it was important to include the minority cultures in the programmes, they could still help the children without exploring the children's family cultures on a deeper level.

It is also important to note that the teachers' beliefs and practices regarding Chinese culture reflect some essential discourses surrounding multiculturalism. On one hand, the teachers appeared to have acknowledged the limitations of monoculturalism as they readily used available Chinese sources in their work with these children (May, 1999; Todd, 1991). On the other hand, they upheld mainstream cultural practices as the key way of working with the children. Possibly the teachers were at a crossroads in terms of their development of multicultural practices. This finding clearly reflects research in New Zealand literature regarding increasing recognition of the limitations of monoculturalism of people from the mainstream cultural group and their reluctance to be fully multicultural (May, 2004; May, Modood \& Squires, 2004). Teachers' practice 
illustrates that EC centres mirror the social practices of New Zealand society. Therefore it is important to acknowledge that EC teaching practice is not only the product of the teachers, but of their engagement in sociocultural activities within and beyond the centre environments. Factors such as teachers' education, home background, their upbringing should all be considered (Banks, 2001). This finding highlights that the learning experiences of the children in this study were influenced not only by the culture of their families and of the centres but also by other cultures, such as their teachers' personal cultures. This idea confirms the point I made before about the multifaceted nature of 'culture' in this research (see section 1.3).

To be addressed here too is the implementation of minority cultures at a level of only celebratory and explicit artifacts, although this was a basic practice aimed at multicultural education (Ryan, 1999; Sleeter, 1995), it had its positive outcomes. The inclusion of some Chinese cultural tools would have given the children a message about the accepted status of their family culture and put them at ease in their new cultural community. As will be noted later in this chapter and in Chapters 6 and 7, the children's motivation to use tools of both Chinese immigrant family culture and those of the EC centres should be evidence of their comfort in both cultures. Woods et al. (1999) consider this to be a natural outcome for children exposed to two cultures. According to Igoa (1995), this could also mean that these children wished to become bicultural and "that longing may in time move [them] to... integrate both worlds" (p.85). The inclusion of accessible and celebratory tools of Chinese immigrant family culture in the community of the New Zealand EC centres, to a certain extent, constituted a basis on which the study children could explore both cultures.

The attempts to include Chinese culture also suggest transformation of teachers' practice. It raises the need to rethink culture, for its evolving, social and context-based nature (Lubeck, Jessup, deVries \& Post, 2001; Vygotsky, 1978). The teachers were changing as a result of changes in their context and their interactions with the changed context. Rogoff's (2003) explanation of the cultural nature of human development is helpful here in terms of how learning has a social nature and results from participating in life. The teachers' transformation of teaching practice could also be the result of the influence of some EC guidelines, particularly Te Whäriki that emphasises the importance of cultural diversity in EC settings. 


\title{
5.3 Chinese immigrant parents' beliefs and practices about child rearing and early education
}

\subsubsection{Serious learning only happens at home}

An important statement by all the study parents was that the children needed to learn serious things, such as words, numbers, drawing, or piano. The parents all said that their children learned these things at home as opposed to in the EC centres.

\begin{abstract}
Amy's mother: I teach Amy words and numbers. She learns Chinese and English with us.

Eden's mother: Children must be taught. I give Eden tasks everyday, colouring pictures, writing English alphabets, Chinese words or numbers.

Jim's mother: $\quad$ I teach Jim words in English and Chinese.

Leah's mother: I teach Leah numbers, words and some important things she needs to learn. Leah attends art classes and ballet classes at weekend too.

Luke's father: Luke has a forty minute piano session each day. He has to sit there all the time because piano is a formal lesson. Although he could not in the beginning back to two months ago, he can do it now. Except for piano, Luke's mother teaches him drawing.

Luke's mother: $\quad$ I draw first and get Luke to learn.

Rick's mother: $\quad$ I teach Rick to write and learn numbers.

Sarah's mother: $\quad$ Sarah does some learning everyday with her sister. My husband teaches them together. Sarah loves learning. She can sing, dance and draw.
\end{abstract}

Xiaohan's mother: I teach Xiaohan some English words and she is learning chess.

The word teach was used in many parents' statements. This indicates that the children experienced being taught in their homes. One can also see from the parents' statements above that in their parental roles, the parents adopted a training practice, by being actively involved in their child's learning and undertaking the role of supervision and teaching (Chao, 1994; Gorman, 1998; Johnston \& Wong, 2002).

The parents reported several reasons why children should learn serious things at home. Firstly, according to all the parents, academic skills were crucial for children's development. With slight differences in opinion about how academic skills could be 
introduced at this stage, all the parents agreed that their children should be given opportunities to develop these skills. Luke's father said that learning must take place now otherwise it would be too late: "you know the beginning decides the end. It is important that a young child takes life seriously or it will be too late".

Secondly, all the parents said that although academic learning was important, it did not seem to be happening in the EC centres, so the family should provide it. Luke's mother said: "Parents are responsible for their children's learning". Amy's mother told me: "Amy has not learned any academic things in the centre" and Eden's mother stated: "Parents cannot rely on the teachers to teach our children". All the participating parents had university qualifications and for them, developing into an educated person was not a big goal for their child but more a natural outcome. For example, according to Luke's mother, "my child should be better than us". Eden's mother said that too: "in my family, everyone has a good education. My child cannot be brought up in another way".

Thirdly, for the parents of Amy, Xiaohan, Leah and Rick, it was important that their children were not behind their peers in China because they might return to live in China.

\begin{abstract}
Amy's mother: $\quad$ Three-year-olds in China know a lot. I don't want Amy to be too much disadvantaged because she may go to China for primary school and it is important that we build good academic foundations for her.
\end{abstract}

Xiaohan's mother: Xiaohan may not stay here too long. I want her to experience everything, in particular, the academic things. If we want, we can just take her back to China and she can easily catch up with the peers there.

Leah's mother: Leah is going back to China probably before she starts school. She will stay there for a while. I want her to learn as much as she can so that she will not be too unhappy because she is not as good as the peers there.

Rick's mother: $\quad$ We are here temporarily. Rick's father is in China. Rick will go back. You know the children in China learn writing and numbers very early and even if we cannot be the same, Rick should learn some.

Leah and Eden's mothers indicated peer pressure to be a reason for them to provide their children with academic learning. 
Leah's mother: $\quad$ My friends do the same. They take their children to classes and teach children many things. I will feel bad if Leah does not have those experiences.

Eden's mother: I always share experiences with other Chinese parents. Their children learn lots of things at Eden's age.

In addition, all the parents believed that there were lots of valuable things in Chinese culture and it was only in homes that the children could learn them.

Amy's mother: Amy is Chinese and we should show her all the important aspects of Chinese traditions. It is particularly important that Amy keeps the Chinese language.

Eden's mother: There are lots of good things in Chinese culture we must let our children know, such as the value of working hard, self discipline. Although he will grow up here, Eden is always Chinese. Nothing can change that.

Jim's mother: $\quad$ Chinese culture is the right guide for us in many aspects. The centre cannot do it so I should show these things to Jim at home.

Leah's mother: The fundamental education is solid in China and it is good that children have a good start so that they can be more competitive.

Luke's father: Chinese culture has much value and we must use that with our children for them to develop proper characters. Chinese children must keep their home language.

Rick's mother: $\quad$ The fundamental education in China is so good that children can take from it some important stuff for life, such as hard working attributes.

Sarah's mother: $\quad$ My husband and I had our education in China. I like the hard working attitude and I want Sarah and her sister to have that.

Xiaohan's mother: The academic emphasis in Chinese culture benefits children.

There seems to be a range of determinants of parents' expectations for their children's learning experiences, which suggest that the parents upheld the Chinese cultural emphasis on academic pursuit, cultivation of proper characters (Huntsinger et al., 2000; Rosenthal \& Feldman, 1996), the use of the Chinese language, and the importance of parents' role in training children (Chao, 1994). Central to the parents' opinions was their recognition of the values of the original culture (Adler, 2001; Li, 2001), and some views showed that they were influenced by their Chinese peers in New Zealand. An orientation to the future can also be detected from the replies that the purpose of learning was to prepare children for later opportunities, such as returning to China or 
adapting to the new society. At a simple level, these orientations indicate that the parents were deciding what they should do with their children. At another level, these decisions reflect the practical issues of parenting in another cultural context. Thirdly, the parents' opinions illustrate that they were all taking responsibilities for their children's learning and development.

\subsubsection{Embracing the mainstream culture of the centres}

All the parents stated that the key purpose for sending their children to EC centres was for the children to learn to function in an out-of-home learning environment in particular and in the New Zealand society in general. Many of them, therefore, intended to embrace their children's centre programmes.

Eden's mother:

Jim's mother:

Leah's mother:

Luke's mother:

Rick's mother:

Sarah's mother:

Xiaohan's mother:

Amy's father:
Eden needs to learn the mainstream culture of the centre, including English and the skills that he can use. Unlike us, Eden cannot be a pure Chinese and if he does not know about the New Zealand culture, he will not have a proper life.

Jim cannot learn 'Kiwi' things from me. It is important that he learns that in the centre.

I want Leah to enjoy herself in the centre by following the New Zealand style of living.

We want Luke to learn New Zealand culture in this centre because he cannot get that at home.

Rick learned to work with people in this centre. It is the most needed skill. At home, Rick can't get the experience of being with people of other cultures. The centre gives him something that he cannot get from home so it is good that Rick fits in.

Sarah should learn New Zealand lives because she will grow in this country. Her centre can give her that.

Xiaohan is very self-centred. She was mean to her friends when she was in China. I hope that she can learn to care, to make compromises and to work with people. The centre is a good place for her to learn these things.

It is important that Amy gets to know how to function in a 'Kiwi' environment.

It appears that the parents expected their children to be experiencing lives in the mainstream way of living through their experiences in the centres; therefore, the parents were willing to cooperate with the centres. This is also reflected in the fact that seven 
out of eight participating children had been given English names by their families. Eden's mother told me: "I don't want others to have trouble using his Chinese names". Jim's mother said: “Jim's older brother said he might be teased if we used his Chinese name". Parents' embracing of the mainstream culture was similarly reported by Ulich and Oberhuemer (1997) in their study of Turkish children's experiences in German kindergartens, where those parents treated the kindergartens as "a ticket that is being bought in advance to give the child a chance in mainstream German education" (p.68). What is particularly noticeable about the Chinese parents in this study is that they placed high value on the ways of being in their new society, and EC centres were seen to be contexts for their children to develop appropriate cultural knowledge. Related to this was the view that the children's experiences in the centres were necessary and worthwhile.

\subsubsection{Holding back their opinions from ${ }^{{ }^{6}}$ 'Kiwi' teachers}

While the parents demonstrated an accepting attitude of their children's experiences in the centres, some commented on the extent to which they thought the children were developing mainstream knowledge. I heard expressions of dissatisfaction about aspects of the children's learning experiences but many said that they kept these opinions to themselves. Following are some typical examples.

Eden's mother:

Amy's mother:

Leah's mother:
Eden speaks too much Chinese at the centre. He might have felt insecure in the beginning and wanted to be with the Chinese people, but it should not continue so long. I can see the centre encourages that because this makes the teacher's work easy. The only thing that Eden learned there was to eat by himself. He became naughtier and once he even lay down on the floor wailing. The centre is too relaxed. There should be more structure. Children must do useful things every day and Chinese children should be helped with English. I feel that the teachers [all teachers] don't do anything with him. Eden manages his own life.

Amy has not learnt much at the centre, not even much English.

The teachers [all teachers] should teach children a bit more.

\footnotetext{
5 In this study, the term 'Kiwi teachers' refers to Pākehā teachers. This is especially so for Amy, Luke and Sarah who only had Pākehā teachers in their EC centres. In some cases, however, I also use this term as a collective term for all the teachers. There will be an indication in the form of '[all teachers]' when Chinese-speaking teachers are also included. Section 5.3.4 specifically focuses on the relationships between Chinese immigrant parents and Chinesespeaking teachers.
} 
Luke's mother: I want Luke to be recognised for his music talent. The teachers do not seem to know that.

Amy's mother was the only parent who approached teachers with her opinions but according to her, it was pointless. She said: "I went to see the manager about moving Amy to the 4-year-old group because she needed advanced learning, but the manager did not help. I just gave up. It is pointless to go further".

Moreover, most parents kept their opinions to themselves and demonstrated an accepting attitude because they were not sure whether the teachers would be interested in what they said due to their different values and beliefs and the parents' membership of a minority cultural group.

Eden's mother: $\quad$ The 'Kiwi' teachers do not talk to me. I don't think they like to listen to me. We are different cultural people. It's important that we don't offend teachers because we are minorities.

Luke's mother: It is not that we do not have anything to talk to the teachers. We don't know whether the teachers would be interested.

Several parents commented that they did not know much about the centre's practice and might not be knowledgeable enough to judge the work of the teachers. This also contributed to their thinking that they should be quiet.

Yet another reason for the parents to keep their opinions to themselves was the lack of time for them to share information with teachers. Xiaohan's mother, Luke's parents, Jim's mother and Rick's mother stated that they would love to be involved in their children's lives in the centres if they had time:

Xiaohan's mother: I am too busy. Sometimes I feel guilty that I just leave Xiaohan there to manage her own life. I should know something about her centre but I cannot.

Jim's mother: $\quad$ I should work with teachers [all teachers]. But I am so busy. At this stage, I just let Jim learn ways around and if there is a big concern, I would talk.

Rick's mother: I have a baby here. I have no time for Rick. I stayed in the centre before I had the baby but could not do it anymore. 
Luke's mother: $\quad$ Luke's dad is busy with his sister at home and I study full time. Even if we want, we have no time to think too much about Luke's centre education.

A further reason for some parents not initiating communication with teachers was the lack of substantial and useful information from teachers.

Amy's mother: The teachers tell nothing except how good Amy is.

Eden's mother: What can I hear from the teachers, especially those Kiwi teachers? They always say good things. We are too different. They may think of our opinions as exaggerations only.

Luke's mother: I know Luke might have trouble settling in. I tried to ask and the teachers said everything was good.

Many parents claimed their children's safety and happiness to be an important focus of the children's experiences in the centres. As long as their children were happy, they did not need to trouble the teachers.

Jim's mother: $\quad$ I do not have a big concern to communicate with Jim's teachers [all teachers]. Some problems are only minor. Jim's happy here, so I'm happy.

Leah's mother: Leah is happy. There may be unfairness but what matters most is that Leah is happy and she is not affected.

Amy's father: $\quad$ Nothing else is more important than seeing Amy happy there. There are problems and we are not happy with the teachers but since Amy's happy, we can just let her go.

Rick's mother: $\quad$ I know Rick is safe and happy. That is important.

Sarah's mother: $\quad$ Sarah seems fine in that centre so I have nothing much to worry about.

Luke's mother: $\quad$ The teachers have so many children to attend to so it does not matter if they cannot do much for my child. The main thing is that Luke is safe.

However, unlike the other Chinese parents, Eden's mother did not think she could subordinate her other expectations to only the safety and happiness of her son. She told me: "The child knows nothing and he can be happy just for being naughty and doing bad things. Eden learnt bad behaviours at the centre but I don't want to express this to the teachers. The teachers cannot change. The centre cannot change. I am looking for another centre for Eden. Eden needs to learn proper behaviours and he should learn 
English". It is evident that the reason for her silence was that Eden's mother perceived herself to be powerless to challenge the centre's status quo. Her response was to seek an alternative way to meet her needs. As noted in the next section, my interview with Eden's mother revealed that although she had spoken with Dawn, the Chinese-speaking teacher in Eden's centre, about her unhappiness with Eden's exclusive play with Chinese-speaking peers, she had not seen Dawn doing anything about her concern. Possibly because of this, Eden's mother said, "The teachers cannot change". As my interview with Eden's mother took place after her conversations with Dawn, therefore, it is likely that she included Dawn as one of the 'teachers', who acted only for the 'Kiwi' community.

To a large extent, the findings here are consistent with what Dyson (2001) reported in her study of home and school communication between Chinese immigrant parents and schools in Canada. She also identified a range of parental dissatisfied opinions regarding their children's learning in schools. As in Dyson's study, the parents here also kept their opinions from the teachers. Although this could indicate, as Dyson also suggested, the cultural and linguistic differences between the Chinese parents and 'Kiwi' teachers, the findings in this section also reveal the parents' dilemmas, stress and their perceived powerless position in New Zealand EC contexts. The complexity of the parents' attitudes to members of the EC culture can be seen as the result of their perception of their minority status and their adaptive strategies to live in the new society (Li, 2001). Although there were consistent positive statements regarding living in New Zealand, with replies such as "the people are quite nice", some also agreed that racism is inevitable in an immigration country like New Zealand (e.g., Leah's mother) and it was therefore important not to do anything that was offensive to people of the mainstream culture, especially teachers of their children (Eden's mother; Luke's mother).

In addition, unlike what is stated in traditional Chinese culture about the clear division of roles between teachers and parents (see section 3.1.2.3), many parents in this study considered their involvement in the centres to be important, perhaps as a result of their dissatisfaction with some learning experiences of their children in the centres, or the 'useless' information they gained from the teachers. However, in response to their life situations and possibly their anticipated experiences as immigrants, the parents chose to 
be quiet, although this was not by preference. This means that changes in life obliged the parents to adopt a reluctant way of living because the parents "struggle[d] to adjust to their visible minority status" (Li, 2001, p.488).

\subsubsection{Communicating with Chinese teachers}

In the centres where there were Chinese-speaking teachers, many Chinese immigrant parents were observed to approach them for conversations, especially Eden's mother, who talked with Dawn quite often. The following is part of a conversation between them.

The mother: "Eden wu fan chi de hao ma?" [How much lunch did Eden have today?] Dawn: "bu shao" [quite a bit].

The mother: " "ta you you he na ji ge zhong guo hai zi wan le?" [Did he play with those Chinese children again?]

Dawn: Eden hai mei you jiang ying yu de peng you" [Eden has not got English-speaking friends yet].

The mother: "zhe bu hao, ta he na ji ge zhong gou xia hai xue le hao duo huai mao bing, er qie ying yu yi dian dou bu jin bu" [Too bad, he learnt some bad things from those boys and his English has not progressed at all].

Here, Eden's mother appeared interested in Eden's life in the centre and she expressed her concern about it as well. Rick and Jim's mothers were also observed to talk with the Chinese-speaking teachers in particular, asking detailed questions about their children's days.

These parents' behaviours with Chinese teachers indicate their interests in the children's experiences in the centres as well as reinforcing the fact that the parents had concerns. The parents' relationships with Chinese teachers made a striking contrast to their relationships with 'Kiwi' teachers. It is interesting to note that although the parents such as Jim's and Rick's mother cited their busy schedules as why they did not communicate with 'Kiwi' teachers, they both made time for conversations with Chinese teachers.

The reasons offered by the parents for their specific relationships with the Chinese teachers are as follows:

Rick's mother: $\quad$ I talk to May just as friends. Since I knew her the first day Rick came to this centre and she was the person for Rick at that time, we built a personal relationship with each other. 
Jim's mother:

Eden's mother:
Ellie knows Jim because we are all Chinese.

I feel comfortable to talk to Dawn because she is Chinese.

These replies agree with Woods et al.'s (1999) finding about immigrant parents' relationships with teachers of mainstream cultures that "parents sought a solution by withdrawing into their own cultures" (p.201). By relating to Chinese teachers, the parents appear to have withdrawn into the Chinese cultural community, possibly for the reasons they mentioned in the section above, that is, being uncertain of 'Kiwi' teachers' interests, diffident to approach 'Kiwi' teachers, and sceptical about the efficacy and outcome of speaking out. It is very likely that the parents felt comfortable to relate to the people of their own culture because Chinese teachers spoke their language and were able to communicate effectively with Chinese immigrant parents. While Eden's mother knew that Dawn did not help her with her enquiries, she still felt comfortable to talk to Dawn because they were both Chinese. Adair and Tobin (2008) reported the same point in their study of immigrant parents in preschools, that for easy communications, "they [immigrant parents] were very clear about their appreciation of the preschool having teachers and other school staff members who speak their language" (p.143). Obviously, the study points out the value of bicultural and bilingual teachers because they were able to assist the parents from minority cultural backgrounds to participate in EC settings.

However, the aspect of being Chinese was commonly exhibited in the Chinese-speaking teachers' use of the Chinese language with the children and their parents. None of the study data indicated purposeful incorporation of Chinese practices in these teachers' teaching programmes with the Chinese children, for example by initiating a Chinese nursery rhyme. One of the Chinese-speaking teachers, May, sang a Chinese song with children in a large group but she did so in response to a colleague's request not her own initiative. Dawn, although she knew of Eden's mother's need, did not bring it to the attention of the teaching team because she did not feel that Chinese parents shared with teachers useful aspects of their children's learning (see section 5.2.1). It is likely that, similar to their Pākehā colleagues, the Chinese-speaking teachers perceived the mainstream ECE to be appropriate for Chinese immigrant children. Another possible reason is that the Chinese-speaking teachers regarded themselves to be also cultural minorities, therefore, they were careful with what they did in order not to deviate from the norms of their ECE centres. 


\subsection{The children's perspectives of learning and developing in two different cultural communities}

\subsubsection{Learning happens at home}

All the children, as illustrated in the following statements, perceived their life schedules at home to be different from what they did in their centres. Their answers to my question regarding what they thought they did at the centre were as follows:

$\begin{array}{ll}\text { Amy: } & \text { Play and learn English with teachers. } \\ \text { Jim: } & \text { Play and wait for mum. } \\ \text { Sarah: } & \text { Do what other children do and play. } \\ \text { Eden: } & \text { Play. } \\ \text { Xiaohan: } & \text { Pay. } \\ \text { Rick: } & \text { Play with others. } \\ \text { Leah: } & \text { Pay and learn something. I should learn English here. } \\ \text { Luke: } & \text { Play and do what other children do. }\end{array}$

Thus the children thought their attendance at the centres had three purposes: to play, to do whatever other children do or to learn. However, they told me that they did the following at home:

Amy: $\quad$ Play, help mum; go to school with my sister; learn lots of things.

Jim: $\quad$ Play, see friends; learn songs.

Sarah: $\quad$ Play with my sister; with some friends; read and write.

Eden: $\quad$ Learn English; play, read stories.

Xiaohan: Play chess, learn Chinese; learn English; play other things.

Rick: $\quad$ Play with my brother; help mum; play with friends; do work everyday.

Leah: $\quad$ Talk with mum; go to library; go to friends; learn piano, draw, play.

Luke: $\quad$ Piano; learn English; watch TV; play. 
So, the children believed they did many more activities at home than at their centre. While at the centre, they experienced a range of learning opportunities which to me as an observer, did not seem to be much less than what they stated happened at home, the children, however, perceived their home experiences to be broader and richer. Perhaps the reason for this perception could be that the home experiences were more focused, memorable and familiar for the children. It is probable too that the parents would have communicated their thoughts to the children regarding the importance of home learning and the limited learning opportunities in their centres. This line of thought can be further supported by the words play and learn used by the children. While learn mainly described their home experiences, play characterised the experiences in both settings. This indicates that the children perceived the EC centres to be a place where they predominantly played, while in homes they learned as well.

Amy was the only child who pointed out that she learned with teachers, which gives an insight into her understanding of what learning entailed. It is very likely that Amy perceived learning to be an adult-organised activity, rather than when she was playing alone or with peers. This explanation might apply to the other children too because in Chinese culture, learning derives from being taught (Wang, Bernas \& Eberhard, 2005). Therefore the children in this study might have aligned their perceptions to the Chinese culture, and to have categorised many aspects of their experiences in the centres in the broad sense of play.

In addition, considering some children's statements about what they worked on in their homes, it is conceivable that the children understood learning to be a focused engagement with tasks. Xiaohan told me: "when you learn, you should sit at a table and do it quietly". Luke said the same thing: "I sit long time when playing the piano". If the children understood learning to be work on adult-organised tasks that required sitting at a table, they would define very few experiences that I observed at the centres as learning.

\subsubsection{Teachers are important in the centres}

In the interviews, when I asked the children "what do your teachers do with you?" they replied: "teachers direct me"; "teachers look after me here" and "I listen to them". 
Furthermore, nearly all the parents described their children's relationships with teachers in this way: "Leah knows to respect teachers because she has been told of the importance of teachers to teach them, watch them and to make sure they behave well. Teachers are like parents to her" (Leah's mother). The parents' and children's statements provided insights into the children's beliefs in teachers as important people.

One feature of teachers that the children appeared to consider as important was teachers' power. According to Corsaro (2005), children see adults "as having ultimate power over their everyday lives" (p.219). This point is important because it draws attention to the children's perception of teachers' power in their relationships with the children, which in turn influenced the children's learning experiences. There was indeed evidence in the children's data that suggested that some aspects of their experiences were anchored in the children's perceptions of the power of their teachers. In subsequent discussions, I have chosen some examples to illustrate this.

The following examples came from my observational field notes in which I recorded two hiding manoeuvres that Luke did to teachers. To me, his behaviours in these situations reveal a tension for the child between retaining his own way of doing things and following those of the teachers. Luke was reluctant to change his behaviours for the teachers, but in the end he did comply.

Luke is jumping on the trampoline alongside two children. The teachers are not with them.

Luke quickly gets off when Jess (teacher) is walking to him.

Luke: "lao shi yao sheng qi le" [the teacher would be upset].

Luke takes off his shoes and goes back to the trampoline again. (Luke/4 $/ 4^{\text {th }}$ visit, $\left.4: 11 \mathrm{pm}\right)$

Prior to the first incident, Luke had been told by a teacher to take his shoes off before going on the trampoline.

On another occasion, Luke brought a box of toys off a shelf. When he was just about to open it, he saw Nicole walking over and quickly put it back, and went to the bathroom. Similarly, observing this incident, I recalled that I had previously seen Nicole telling Luke not to touch that box in response to his attempt to take it. In the first incident, Luke said to himself "the teacher would be upset" when he took off his shoes. It is very clear that Luke did not want the teachers to know what he was doing, so he stopped. 
Sumsion (2005) described preschool children's perception of teachers as having power, and that teachers have techniques to make children conform to rules. Similarly, in this research, the reason for Luke's hiding of his rule-violating behaviours from his teachers could also be his awareness of the teachers' power to regulate his behaviours.

One teaching technique that Luke had experienced was when a teacher looked 'upset', she moved him from what he was doing to a place where he needed to sit for a few minutes on his own, such as time-out. It is possible to infer that Luke learned from these experiences that he would be in trouble if he upset his teachers. Because of this, Luke used hiding as a strategy to get out of trouble.

Overall, Luke's behaviours with teachers in these scenarios highlight two points: Luke intended to resist the teachers' control (Cobb, Danby \& Farrell, 2005; Corsaro, 1997, Hoogsteder, Maier \& Elbers, 1998) and he knew the importance of teachers regulating his behaviours. These points illustrate that Luke was in a dilemma. However, when he changed his preferred behaviours, Luke displayed his submission to the teachers possibly because he acknowledged the teachers' authority and the importance of submitting to it, thereby avoiding trouble.

The teacher's importance for the children was also noticeable in Amy and Sarah's data which provided a record of their careful following of teachers' instructions. As identified in the following scenario of a 'tidy-up' activity in which children and teachers worked together to clean the centre, Amy actively took part in all of it, while many children in her centre did not participate.

Iby (Amy's teacher) (calling): "tidy up time”.

Amy is running around outside and quickly jumps inside, bending down, picking up things on floor.

Iby, Mary and Fiona, three teachers are tidying up and a few children are helping too. Amy calls to Iby, who is standing close to her: "Iby, me tidy up" Iby: "good girl".

When the room is clear, Amy goes outside to continue helping tidy up. (Amy/ $4^{\text {th }}$ visit, 11:36am)

Amy seemed enthusiastic and happy with what she was doing with the teachers and some peers. The words "Iby, me tidy up" reflected the child's intention to be acknowledged and liked. Igoa (1995) reported immigrant children's eagerness for 
affirmation from teachers because they knew teachers were important to them. This could explain why Amy was so responsive to teachers.

Sarah also seemed to closely follow teachers' instructions. The example below illustrates her helping out at tidy-up time, responding to teachers and being among only a few children who did this.

Sarah is in the sandpit digging sand when Casey (teacher) calls: 'tidy up time'. Sarah quickly stops, taking the spade to a bucket. She then picks up the toys in the sandpit and puts them in the bucket. After the sandpit is clear, Sarah runs to help lift a table with some peers and Casey. Sarah then goes to the storage room, helping Anne (teacher) put things in order.

Anne: "thank you".

Sarah smiles.

Sarah walks to the block area, putting the blocks away. (Sarah/3 $3^{\text {rd }}$ visit, 11:55am)

Sarah looked happy when she was praised. As with Amy's liking for teacher affirmation, Sarah appeared motivated because Anne acknowledged her contribution. In the study, Sarah did everything that teachers asked. Sarah's mother said: "Sarah really wants to be liked". The teachers in Sarah's centre appeared to be friendly with Sarah, so Sarah might have believed that the teachers liked her which would explain why she took every opportunity to obtain teacher approval. Furthermore, as will be discussed in Chapter 7, Sarah had very few interactions with peers, so she might have been trying to form relationships with teachers to compensate for her lack of interactions with peers. One strategy she was using was to do whatever the teachers asked her to do.

As a child who has been attending her EC centre for ten months, Sarah could have relied on a degree of intimacy with her teachers to help her settle into the programme. It is common for nearly all children, regardless of their cultural backgrounds, to interact more with teachers than with peers when they transition from homes to other education settings (Dalli, 1999; Igoa, 1995; Ladd, 1996). Thus, what Sarah demonstrated with her teachers might not necessarily be a behaviour related to her home culture, but one to her stage of settlement in the EC centre.

Amy regarded teachers as important because teachers could teach. Amy told me: "I need to learn here". Her mother said during our interview: "Amy knows the importance of teachers to teach". Given that Amy had previous experience with teachers in China, 
who, as Amy's mother said, "did lots of teaching", she might have built this image of teachers as people who teach, which would have contributed to Amy's behaviours with teachers. The next excerpt occurred during a routine 'mat time' session, in which teachers and most children were sitting on the floor and involved in some learning activities. Amy placed herself in the front row, and closely watched and listened to Mary, the teacher who was positioned facing the children, telling them a story and facilitating the discussion with them. My field notes recorded:

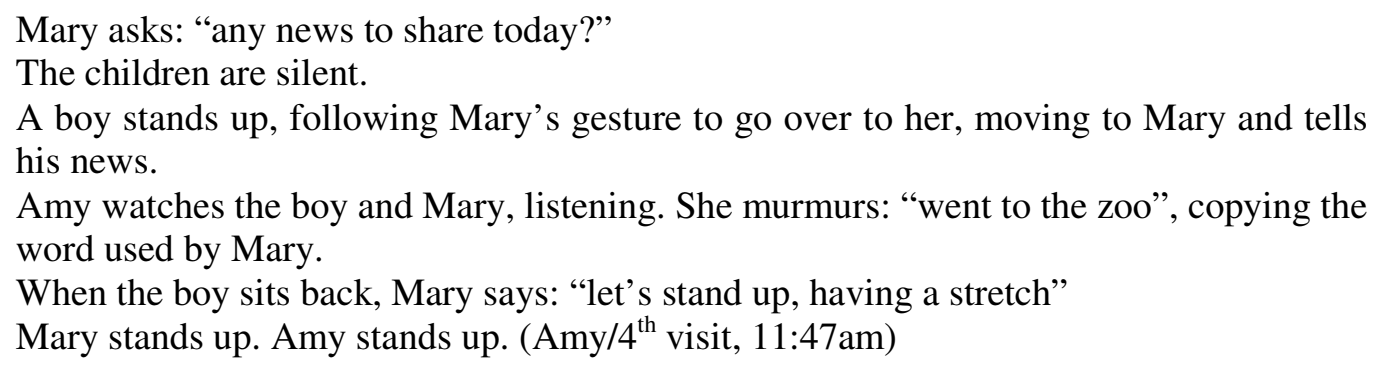

Amy was observed to pay attention to Mary and followed her throughout the mat time session. Although she did not offer a contribution, Amy listened, watched and followed Mary and the group. When Mary talked, Amy quietly copied "went to the zoo" and in doing so, indicated her active engagement with Mary.

Amy's behaviour described above also characterised an important feature of learning theorerised in Rogoff's guided participation. Amy was learning through observations as an onlooker (2003). Her role in actively following Mary was critical in developing these learning experiences.

Amy's enthusiasm for teacher-organised learning activities was consistent throughout my data gathering period. Amy's active engagement with teacher-organised learning activities demonstrated that she made a connection between teachers and learning.

The examples that illustrate the children's high regard for teachers are typical of the attitude fostered in Chinese culture (Yau \& Smetana, 2003). All of these behaviours are consistent with Chinese cultural conventions of children-teacher relationships. The data, thus, suggest that the Chinese culture, particularly regarding the importance of teachers, mediated the learning experiences of the children in non-Chinese learning settings. 
On the other hand, as I have explained above in relation to Sarah's behaviours with her teachers, it is possible to infer too that these children attempted to be close to teachers because of their stage of settlement in the EC centres. It takes time for newly arrived children to understand how things work in the new environment and to participate easily in the learning activities there. It is reasonable to expect that the children used their teachers to establish "a known reference point within an unknown environment" (Dalli, 1998, p. 65), because the teachers focused on them and provided them with time for interactions (see section 5.2.1).

\subsubsection{Being in the centres is a commitment}

In the interviews, all the children implied that they needed to find ways to settle in the centres. For example, Jim said: "I must stay here because mum needs to work". Xiaohan told me: "I'm big enough to attend school". Rick commented: "Mum has my little brother to look after. I must be here". Amy stated: "Everyone else comes to childcare. I need to learn English". The children thus conveyed a message that being in the centre was not a choice, but a commitment or necessity. Therefore, the children were observed to have made efforts to settle themselves there. There will be discussions in Chapters 6 and 7 about the children's endeavours to communicate, make friends and learn about the centre's programmes.

There were also plenty of illustrations of the study children involved in many kinds of learning activities available in the centres. I did not observe any examples of the children showing a particular dislike of any of the experiences. Although the teachers indicated water or messy play were generally loathed by Chinese children, and some literature has supported this (Huntsinger et al., 2000), many children in this study were observed engaged in everything that their peers and teachers were doing.

Given the strong expectation of the Chinese immigrant parents for their children to develop 'Kiwi' knowledge and life experiences, and the teachers' espousal of a childcentred pedagogy which led them to provide special attention to the Chinese immigrant children, the children's commitment to settle in the centre can be seen as the outcome of 'guided participation' by both teachers and parents. For example, the children could have learned from their parents that it was important to try out things, given that hard working was a highly valued cultural behaviour of their families (see section 5.3.1). It 
was also clear from the parent interviews that the Chinese immigrant parents were very willing to cooperate with the 'Kiwi' culture. On the teachers' side, their special focus on attending to the children's needs and experiences in the centres would have likewise encouraged the children to try hard to settle. In this way, the children's action of settling can be seen as the outcome of the convergence of the guiding actions of both the culture of the Chinese immigrant families and the mainstream culture of the EC centres. This convergence of the two cultures can thus be considered as creating the possibility of intercultural relationships by the children. As illustrated in Figures 6.1, 6.2, 7.1 and 7.2, as well as discussed in section 7.1.2, the children created this possibility in a number of ways: bridging the two cultures; keeping the family culture apart from that of the centres; and keeping the family culture away from the centres. Figure 5.1 illustrates this convergence of cultural practices and beliefs from the children's different cultural settings.

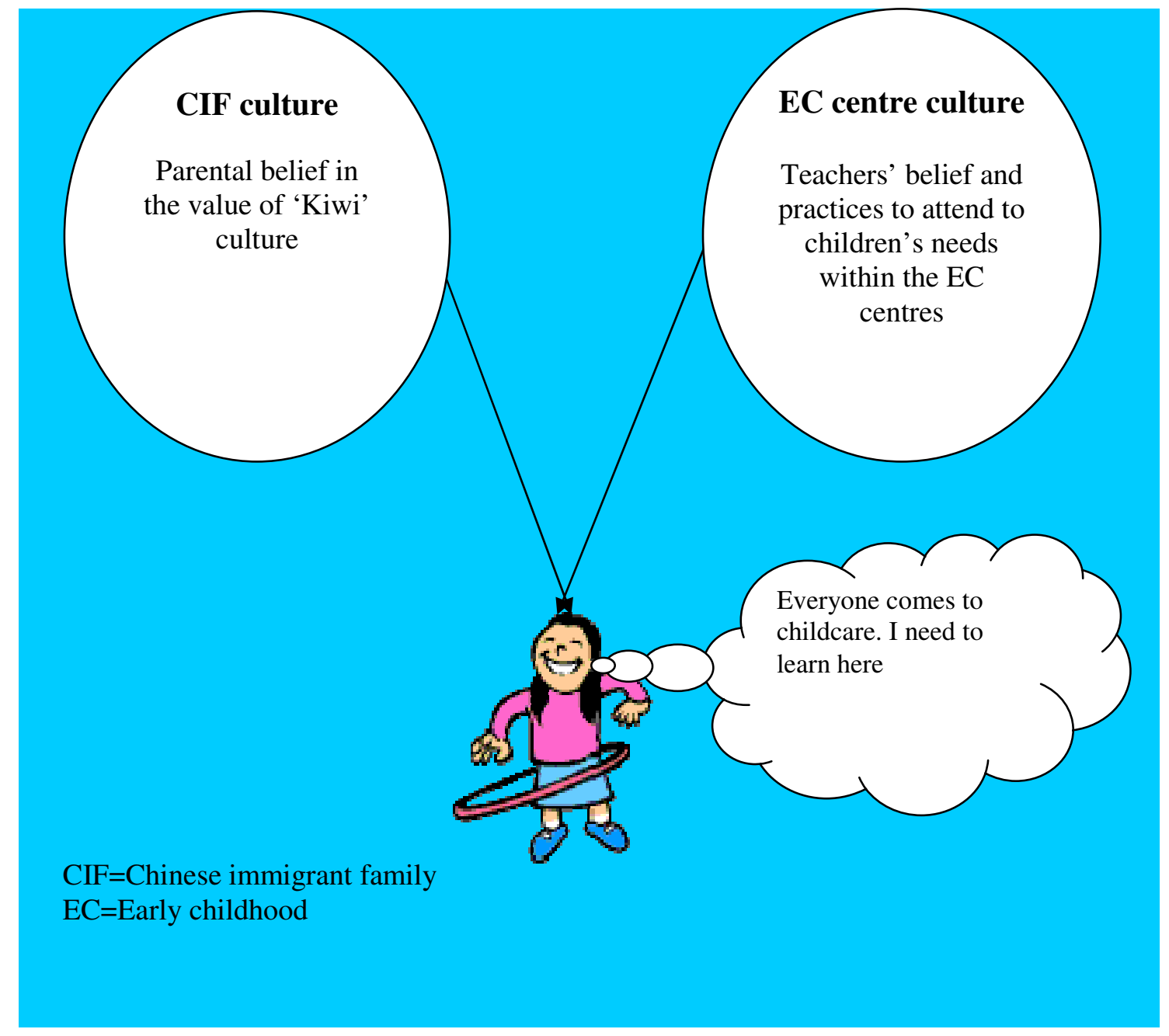

Figure 5.1: Convergence of cultural practices and beliefs from the children's two cultural communities to guide children to settle in the centre 
Another reason for the children making a commitment to participate in the centres could be the result of their 'transformation of participation' (Rogoff, 2003) as an inevitable consequence of becoming a member of the centre community. It is conceivable that the children embraced certain aspects of the cultural practices and developed interests in some activities in the centres. Therefore, they wanted to explore and try things out in pursuit of their own interests and wishes.

One could also argue that the study children were actively involved in the learning programmes of their centres because they did not want to be different from their mainstream peers. Given that "by the age of three, young children already are sensitive to differences" (Adler, 2001, p.297), and the strong upholding of Chinese culture by the parents, the children are likely to have been aware of the difference between their culture and that of many peers in the centres. It is also possible that the children wanted to be accepted by the mainstream group. This is because immigrant children are conscious of their differences and are afraid of being teased (Klein \& Chen, 2001), and Chinese immigrant parents tend to inform their children of the disadvantages of their differences in a new society ( $\mathrm{Li}, 2006)$. The study children tried hard to settle so that they could minimise potential disadvantages that resulted from their differences.

\subsubsection{Parents are passers-by in the centres}

Because many parents chose to leave the centres promptly as they dropped off and collected their children, the children might have concluded that their parents should not stay in the centre but leave immediately. This could be why in the following excerpt, Leah told her mother to leave in a very insistent manner.

Leah walks in, carrying a bag on her shoulder and her mother is following her behind Leah walks to Mary (English girl): "Hi, Mary", and pats Mary on her arm. Mary turns to her and holds Leah's hand. Leah turns her eyes to the door where her mother is watching her. Leah waves 'goodbye' to her mother. Leah's mother smiles at her but does not leave. Leah says to her mother: "mum, zou zou" [Mum, go, go]. The mother gives her a 'leave me' gesture, but Leah does not leave her mother alone. Instead, she walks to her and pushes her away. She stays by the door, watching her mother leave then goes back to the room. (Leah/ $/{ }^{\text {rd }}$ visit, 9:51am)

Leah's mother was the only parent in this study who was observed to stay for a short while in the mornings, because as she said: "I used to come and leave because my work started early. Now I have a late start and want to have a bit of time at the centre". However, Leah did not allow her mother to stay. On the first visit, her mother watched 
Leah from the window of the hall when Leah sat on the mat with her peers. On that occasion, Leah looked agitated and kept waving 'goodbye' to her mother until she left. Leah told me "my mother should go to work". This child, thus, implied that her mother did not have a place in the centre. In a similar way, she took her mother out when the mother was talking with me and said: "mum, go". It is possible that since Leah was not used to having her mother present in the centre, she felt uncomfortable with this.

Amy's mother was also trying to avoid her one day when she brought Amy's jacket to the centre. The mother left the jacket with the lady in the office because she said: "I cannot go to Amy's room. The other day I came, Amy was very upset when she saw me. Amy does not want us (her and Amy's father) to come to the centre during the sessions".

The behaviours of these children foreground two issues: firstly they wanted to change their focus from home, or their mothers, to the centre, as confirmed by Woods et al. (1999); and secondly they did not think their mothers had a place in the centre. It is likely these children conceptualised the roles of the adults in the centres, according to what they had seen there.

\subsection{Chapter summary}

This chapter has located the study children in their relevant contexts by presenting a summary introduction of them and then examining the opinions of their teachers, parents and the children themselves regarding their learning experiences at home and at the centre.

The summary introduction of the children provided some insights into the individuality of each child. This was intended to facilitate the reader's understanding of the discussions in the following chapters as these related to the experiences of each individual child which would then contribute to understanding the whole research cohort.

The examination of different participants' opinions was in line with (i) the underpinning theoretical position of sociocultural theories that recognises the influence of culture on learning experiences of the children; (ii) consideration of variation in practices across cultural communities and the transformation of participation; and (iii) the concept of 
'learning communities', to view the EC centres as a site that involves "people who are related to one another in ways other than being the givers and receivers of skills or knowledge" (Goodnow, 1993, p.373). These theories were then aligned with the context of diverse cultures in New Zealand EC centres, generating ideas about how diversities were manifested and operated in those settings.

The sociocultural contexts of the children were described using the data of interviews with New Zealand EC teachers, Chinese immigrant parents and the study children, as well as my specific field notes. This information has also set the scene for the discussions in the subsequent chapters of the children's learning experiences in New Zealand EC centres.

Through an investigation into the beliefs and practices of the people in the Chinese immigrant children's families and their EC centres, this research identified some important components of the children's sociocultural contexts: (i) the children's learning attitudes, their commitment to settling in the centres, and their high regard for teachers; (ii) Chinese immigrant parents' practice of the traditional Chinese approach of home training of children and their cooperation with the mainstream culture of the EC centres; (iii) and the mainstream cultural beliefs and practices of New Zealand EC centres which include teachers' focus on children, their approach to treating these children same as all the other children, and teachers' attempts to bring in certain aspects of Chinese culture.

According to the information in this chapter, the study shows the power of culture, including the power of both the original culture and the New Zealand EC culture. While the parents and children were largely influenced by their original cultures, they intended to adopt the mainstream culture of New Zealand, to gain "adaptive strategies" (RoerStrier \& Rosenthal, 2001, p.220) and to live a new life. This is similar to the Chinesespeaking teachers, who were inclined to follow the mainstream culture of New Zealand ECE, but not their home culture-oriented learning and teaching approaches. On their part, New Zealand EC teachers also made some attempts to bring elements of Chinese culture into their teaching practices. 
A further aspect of the findings, which relates to the sociocultural contexts of children's learning and development, was the way in which each culture was represented in the children's contexts. Simply put, the two immediate cultural communities of the study children co-existed in the EC centres, and there is evidence that certain aspects of the Chinese immigrant family culture were able to converge with those of the New Zealand EC centres.

The chapter has also found that all the adult participants attributed more importance to the mainstream culture of the EC centres than to Chinese culture, as a vehicle for the children's future success in New Zealand society. The Chinese parents kept their own culture mainly as a tool to use at home, and the majority of teachers included only accessible and celebratory Chinese cultural practices in their teaching programmes. The adults seemed to have reached a tacit agreement that in the centres, the children needed to focus on learning about the mainstream culture. Thus the children were in a context where the important adults in their EC centres would not provide them with many experiences based on their family culture, unless there were ready and accessible Chinese cultural tools, such as Chinese-speaking peers or teachers in the centres.

During the research, another noticeable feature was the lack of observable input from the children's parents to the children's learning experiences at the EC centres. This phenomenon was clearly related to the lack of interaction between the parents and the 'Kiwi' teachers and appeared to be influenced by several factors: their beliefs and practices about child rearing and early education; ways of communication; time pressures; attitudes of the 'Kiwi' teachers, and of their Chinese-speaking counterparts to Chinese immigrant parents and children; teachers' embracing of the mainstream pedagogy; and parents' perceptions of their roles and their 'minority' status. Although some variation in educational practice between the children's homes and the centres was identified from their replies to my interview questions, it was not articulated by either parents or teachers. This could be because of the absence of signs of ongoing meaningful interactions between the parents and 'Kiwi' teachers, and the limited attempts that Chinese-speaking teachers made to help their 'Kiwi' colleagues to relate to Chinese immigrant parents. This led to the teachers' use of few Chinese cultural tools and their generalization of the children's family culture to be simply 'the Chinese culture'. Because the parents had specific needs and expectations (see section 5.3.3), the 
teachers' cultural generalization probably contributed to their unresponsiveness to the parents and the children. Regarding the concepts of learning communities and cultural diversity, it could be said that learning communities which acknowledge, affirm and integrate cultural diversity did not develop, or were only beginning to form, in the EC centres.

The lack of parental input into the learning experiences of the children in the centres is a concerning finding when one considers an underlying principle of Te Whāriki, which states that "the wider world of family and community is an integral part of the early childhood curriculum" (Ministry of Education, 1996, p.14). How can teachers work under such a principle with Chinese immigrant children, when the families of the children are an 'outside' feature of the children's learning experiences in the EC settings? While the exploration of the children's learning experiences in this research did not aim to critique teachers' practice, a specific issue arises about the contradiction between the teachers' aspirations to support the Chinese immigrant children and their practices in the absence of Chinese immigrant parents' input. This raises significant implications for the education of New Zealand EC teachers in relation to cultural diversity. This research found that the sociocultural emphasis on the importance of the family in Te Whariki was not actively implemented in the teachers' practice within the study.

The following two chapters, Chapters 6 and 7 discuss the learning experiences of the study children based mainly on the field notes gathered during this study. The discussion will focus on the children's use of the languages and their learning experiences with peers. 


\section{Chapter 6: Chinese and English: Languages as a tool to act upon the world}

\subsection{Introduction}

This chapter considers what we might learn about Chinese immigrant children's learning experiences in New Zealand EC centres through investigating their use of the two languages, Chinese and English. The chapter addresses the first key question of the study:

1. How do Chinese immigrant children experience learning and development in New Zealand early childhood centres?

In addition to interrogating the data to answer this question, this chapter pays particular attention to the first sub-question of the first key question, which asks:

a. In what ways do Chinese immigrant children experience the English language?

This focus on language is grounded in the sociocultural premise that language is a cultural tool that mediates learning (Vygotsky, 1962; 1986). On the basis of this premise, it is essential to note that instead of focusing on language as a phenomenon for linguistic analysis and thus considering it as a system of rules with syntactic, phonetic and semantic features, the analysis in this chapter treats language as a dimension of learning and development that the children experienced when acting upon their world.

Although the initial sub-question addressed in this chapter concerned the children's experiences with the English language, once the data were analysed, it became clear that it would be useful to extend the focus to include both languages in their centres. This is because, while English was the key language in all the centres, six of the eight children, that is Leah, Jim, Rick, Xiaohan, Eden and Amy had Chinese peers and Leah, Jim, Rick, Xiaohan and Eden had Chinese teachers too. In these centres, Chinese was commonly used as a means of communication among them. In comparison, Luke and Sarah were the only people who could speak Chinese in their centres, but my presence led them to speak it with me. Furthermore, although the children had varying confidence with 
English, they were all described by their parents to be competent with Chinese. Information about the language backgrounds of the children can be found in Table 5.1.

Therefore it became clear that the Chinese immigrant children's learning experiences in their EC centres occurred in the context of both English and Chinese and that to explore only one language would not provide a realistic picture of their lived language experience.

Thus the initial sub-question was re-phrased to become:

In what ways did the Chinese immigrant children experience Chinese and English?

The framework for presenting the findings is derived from the children's language choices. These self-selected experiences with a language/s indicate the relationship between the children and each language, and the children's attitudes towards the language/s (Mickan, 2006). Further, the children's language choices could also explain how they perceived communication situations, including the people with whom they communicated and the role of the languages in their learning settings (Ledesma \& Morris, 2005). The way this chapter is organised, therefore, also clarifies the sociocultural nature of the children's language experiences (Vygotsky, 1962; 1986).

This chapter is divided into three sections:

(i) code switching between English and Chinese;

(ii) experiencing the Chinese language; and

(iii) experiencing the English language.

\subsection{Code switching between English and Chinese}

There is evidence in the data from all the children that they used both Chinese and English in their centres and many of them mixed the languages in their speech. As mentioned above, two circumstantial factors seemed to have contributed to this phenomenon. Firstly, in all the participating centres, English was the key language for communication, and secondly, all the children could speak Chinese and many 
participating centres had other Chinese speakers. Within these contexts, the children used the two languages interchangeably among themselves, with their peers, teachers and when speaking to me. The literature suggests that code switching can be a purposeful and strategic behaviour in accordance with speaking contexts and speakers (Beardsmore, 1986; Canagarajah 1995; Reyes, 2004), or a representation of speakers' creative interaction between the languages (Bhatia \& Ritchie, 2008). The analysis of dual language use of the children supports both views. Some data are provided below to illustrate how the children switched languages in a range of learning contexts. I argue that these behaviours indicated their incorporation of both languages in their speech and their awareness of the speaking contexts and speakers.

The following table presents instances when the children were observed using both languages when talking to themselves in private situations. 


\section{Table 6.1: The children's dual language usage in private situations}

\section{Code-switching data}

$\operatorname{Jim}(3: 1 / M)^{6}$ is playing with water in the water trough. He sprinkles water onto the ground. Alongside him are three other children, who all play on their own. The children do not interact with each other.

As he plays, Jim laughs: "water, water jiao di di" [water watering the ground]. $\left(\mathrm{Jim} / 4^{\text {th }}\right.$ visit, $\left.2: 10 \mathrm{pm}\right)$

Luke (3:3/M) is making a 'Christmas card' with his teacher Nicole and three peers at a table. Nicole is watching the children making the cards.

Luke is drawing on his card with a grey crayon: "wo yau zuo yi ge green shu" [I need to make a green tree]. Luke reaches the crayon box for a green one. (Luke/ $3^{\text {rd }}$ visit, 10:29am)

Amy (3:6/R) runs with a ball: "stop, stop, wo rang ni stop" [stop, stop, I tell you to stop]. (Amy $/ 3^{\text {rd }}$ visit, 4:12pm)

Rick (4: 5/AC) is reading a book in the family corner where two children are playing animals on the floor nearby. A boy picks up a cow and throws it in the air. Rick stops reading and watches him: "ta bu zhe dao zen ma play animals" [he does not know how to play with animals]. (Rick $/ 4^{\text {th }}$ visit, 9:12am).

\section{Speaking contexts Function of language use}

When Jim is playing, Jim speaks to himself, alongside some peers, verbalizing what he is doing. who do not interact with Jim.

When Luke is drawing in parallel with three

Luke speaks to himself, indicating to himself what he needs to do.
When Amy plays with a ball by herself.

When Rick is watching his peers to play. peers and a teacher who is sitting with them.
Amy seems to be treating the ball as a conversational partner.

Rick speaks to himself, reflecting on action that he has just taken

Despite the absence of other people in the contexts within which the study children produced this body of speech, all these utterances included the use of the two languages; in other words, in all cases the children moved between Chinese and English while they were engaged in private speech, or 'talking to themselves'.

\footnotetext{
6 The numbers and letter/s in bracket after each child's name respectively represent the child's age and English language ability. '3.1' means three years and one month. 'M' means 'minimal'. 'R' means 'routine' English. 'AC' means almost competent (see Table 5.1).
} 
If one accepts the argument that code switching, or a concurrent use of two languages, is a purposeful and strategic behaviour, then the children's actions would have to be interpreted as goal-seeking behaviours: that is, they created the speech events to achieve certain purposes. One might argue, for example, that a purpose for the children in the situations above was to make meaning of what they were doing. Vygotsky (1986) argued that private speech played an important role in helping mediate thinking processes for children, and in all the events here, when the children were talking to themselves, their speech could have acted as "an instrument of thought" (p.16). Also, Barnard (2003) suggested that private speech serves as a tool for language "rehearsal" (p.171) and "language play" (p.172), indicating that the children could have been practising or playing with the languages when talking to themselves. In section 6.2.3, there are further examples of private speech, and I argue that they indicate the children's intention to practise English to themselves. Through those examples I discuss the children's purposeful attempts to experience the two languages. The private code switching above could also suggest this. In his account of code switching, Beardsmore (1986) categorised two types: the linguistic change of codes and the social change of codes. The dual language included in the examples above is likely to represent Beardsmore's linguistic change of code, because when the children practised or played with the languages, the code switching emerged in the form of words and phrases.

A further analysis of the data in Table 6.1 also shows that the children's speech was created in a pattern of language mixing within a single sentence. These communications seemed to enable the children to play a creative role in making verbal interactions. Bhatia and Ritchie (2008) offer an interesting perspective on this in their account of what they call "the cooperation... and coexistence of the bilingual's two languages" (p.10). They claim that using two languages in a single sentence may not necessarily represent a speaker's intention to mark a boundary between the languages, as in code switching, but simply provides an example of integrating elements from different language codes into the one utterance. Therefore, it is possible that the children were switching codes between the languages, but it would be premature to make large claims for this, because it could overlook the creative nature of young children and the flexibility inherent in languages. 
Overall, examples of code switching - or of children's verbal interactions in which both English and Chinese linguistic elements were used- in private situations illustrate that languages did function as a tool that helped the children to implement thoughts and actions. Despite their differing abilities with the English language, all the children attempted to use and practise it together with their home language in self-oriented situations. The children's communications in English were facilitated by their bringing Chinese into the speech events. It is likely that the children saw the English language as a useful tool that could be employed in the same way as the Chinese language.

In some situations, the children used both English and Chinese with Chinese peers. Amy (3:6/R) gave an example of this:

Amy sits at the book corner on her own. Ken, her Chinese-speaking peer is walking around nearby. Amy holds up a book and calls up: "Ken, Ni yao kan zhe ge ma? Hao da de yi ge da chuan, big" [Ken, do you want to read this book? A big ship is there, big]. Ken comes, sits with her, reaching for the book.

Amy removes Ken's hand: "Ken, no touching, zhe shi wo de" [it's mine]. (Amy/3 ${ }^{\text {rd }}$ visit, 3:02pm)

As in the example in table 6.2, Amy incorporated single English words into her Chinese utterances, this time not to herself but to a Chinese peer. Her use of the 'no touching' phrase appeared to serve the purpose of rehearsal because this was the only time I observed her using it, which also suggests that it might have been new to her. This thought could be further supported when considering that Ken appeared to be beginning to learn English, so that he might not have understood 'no touching'. Thus, if Amy's intention had not been to communicate with Ken, she must have used the phrase mainly for herself as practice. Amy's speech here, therefore, functions in a similar way to private speech. In saying so, what should not be overlooked is also the body language that Amy used in removing Ken's hand from the book. Perhaps Amy did this as a communication strategy when she was not attempting to share a common language with Ken, or perhaps Amy supplemented her verbal language with an action that Ken could understand, when she realised that her verbal message did not work and she wanted Ken to understand her. Given that the interaction occurred as a result of Amy calling up Ken, Amy might want to create a 'real' communication context to practise English even if she knew that Ken could not reciprocate. 
Another possible explanation is that Amy was aware of Ken's need to learn the phrase and she wanted to teach it to Ken. This interpretation is supported by Amy's mother's statement during the interview that Amy habitually engaged in dramatic play as a teacher with her toys as pupils. 'Guided participation' (Rogoff, 2003) includes the strategy of children moving to a guiding and teaching role with peers when they master a specific skill, and it is possible that Amy thought she had mastered an English phrase and could therefore teach it to Ken. Related to this hypothesis also is that Amy was familiar with the 'taught' aspect of learning. Here, we can see the structured and formal training practices of Chinese culture come alive (Chao, 1994).

In the following excerpts, it appeared that the dual language usage occurred for social purposes. 
Table 6.2: Dual language usage in social contexts

\author{
Data \\ $\operatorname{Jim}(3: 1 / \mathrm{M})$ speaks to \\ Wayne (Chinese peer) at the \\ drawing table: "bi zai na" \\ [the pen is there], pointing \\ to the pen box. \\ An English-speaking girl \\ comes and sits at the table, \\ next to Wayne. Jim turns to \\ her: "pen", pointing to the \\ box of pens. \\ $\left(\mathrm{Jim} / 5^{\text {th }}\right.$ visit, 2:26pm)
}

Rick (4:5/AC) is making dough at the dough table. Alongside him is a boy. They do not interact with each other. The teachers are elsewhere. Rick makes something. He stands up and walks to his Chinese peer, Ben. Rick shows Ben his dough creation: "see, dinosaur".

Ben makes a look at Rick's creation: "wo k an guo hao da de kong long"

[I once saw a big dinosaur]. Rick: "wo men jia jiu you da kong long" [I have a big one at home] (Rick/ $1^{\text {st }}$ visit, 10:04am)

Leah (4:6/C), watching Mary (English speaking child): "you got to hold this one", shows her a paper bag.

Leah turns to Fang: "ni jiang zhe ge fang jin qu" [you put this one into the bag], giving a teddy bear to Fang.

Leah then asks: understand?, turning to Mary (appears to seek Mary's response) and then turns to Fang: en? (Leah/2 ${ }^{\text {nd }}$ visit, 2:56pm)

\section{Speaking contexts \\ Speakers}

Jim, alone, sat at a table, drawing a picture. Wayne joined Jim, by sitting down across from Jim. Jim saw Wayne coming to the table. He spoke to Wayne.

Rick worked away from Ben initially but when he finished making a dough product, Rick walked over to Ben and showed Ben what he had made, when the conversations occured.

Leah, Mary and Fang went to the family corner. They were sitting on a bench for 12 seconds then Leah left the seat, to fetch toys from the toy bucket and bring them over to Mary and Fang. After sitting back on the bench between Mary and Fang, Leah picked up a paper bag from the floor and handed it to Mary. This is when the conversations started.
With a Chinese person and an English person

With a Chinese peer who understands English

With an English speaking peer who understands no Chinese

With a Chinese peer who does not know much English 
As with the examples in Table 6.1, regardless of their differing English abilities, the children here all moved between two languages to suit the needs of the people they spoke with, to make themselves understood. While in Rick's example, he began using English with a Chinese peer Ben, Rick switched to Chinese when Ben responded to him only in Chinese. The other two children changed the use of the languages definitely to meet the needs of their speakers because in both cases, the people they spoke with only understood one language. This type of language change can only be interpreted as indicating the children's intention to express ideas effectively (Baker \& Jones, 1998; Reyes, 2004). The children created "language boundaries" (Baker \& Jones, p.38) based on knowledge of when a language could be used. It is clear that the speech in these examples was created for social and communicative purposes. Again using Beardsmore's (1986, p.49) definition of the "situational shifting", the children here were engaging in 'situational shifting' because they shifted the language according to the social contexts and speakers.

Interestingly, the examples here illustrate both types of code switching as defined by Beardsmore (1986). In private situations, code switching was more likely to serve the children's need to practise and play with the languages whilst in the social contexts, the children switched languages for communication and social connections. There is evidence that, despite some children's minimal English abilities, they made use of that language.

These examples indicate the children's abilities to differentiate the languages, to connect them, and to select the use of languages in line with contexts, as well as the children's desire for, and pleasure in learning to use the new language. The children's interchangble use of the two languages discussed so far corresponds to the two functions of languages that Vygotsky identified: language completes thoughts and also serves specific social practices.

The analysis of the interactive aspect of language also highlights the role of language in facilitating the process of boundary crossing for the children, and in acting as complementary tools or "boundary objects" that interconnected the learning contexts of the children (Star \& Griesemer, 1989; Wenger, 1998). Such a viewpoint does not deny that languages are distinct entities, an idea from the code switching perspective, but it 
also takes the view that languages are able to be integrated and they are a source of creativity.

Furthermore, underlying the Chinese children's use of both languages is the practical function they established for the Chinese cultural tools within those of the centre. By changing between the two languages, the children demonstrated an awareness of the coexistence of two cultures in their lives. When associating one language with the other, the children connected the two cultures.

The figure below illustrates another example of the convergence of the two cultures through the children's use of the two languages in a single speech event. This intercultural possibility emerged when the children used the Chinese and English languages as common and simultaneous communicative devices to effectively join the two cultures together.

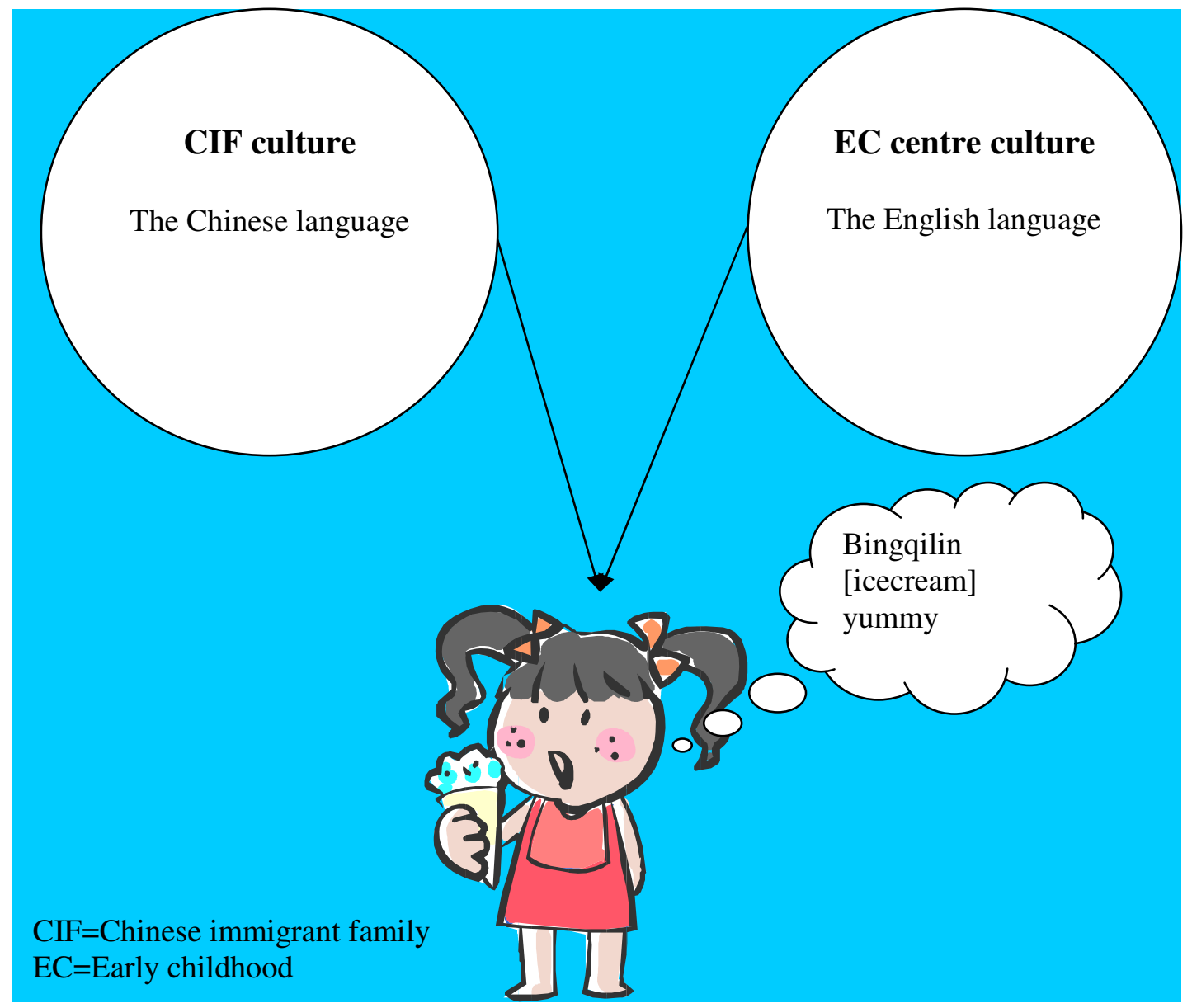

Figure 6.1: Chinese and English as common and simultaneous communicative devices to converge the two cultures 


\subsection{Experiencing the Chinese language}

\subsubsection{Seeking a sense of being together and belonging with Chinese speaking people}

According to de Haan and Singer (2001), togetherness is a feeling of being close to others, in recognition that they understand one another's needs. Inherent in this is a certainty that it is good to contact others and do things together. van Oers and Hännikäinen (2001) remind us that the key indicator of a sense of togetherness is the affective engagement with others in groups. A consequence of togetherness is the formation of a sense of belonging to each other. Osterman (2000) claims that people make efforts to develop group membership to pursue their desire for a sense of belonging.

In this study, I found that instances of the study children's enthusiastic engagement in group activities often occurred when they were interacting with Chinese peers and teachers. The use of the Chinese language was a frequent feature in these activities. In many situations, the children used Chinese to contact Chinese peers and to do things together with them. Some typical examples are shown in table 6.3 below: 
Table 6.3: Using Chinese to contact and seek togetherness with Chinese peers

\section{Before being together... $\quad$ Seek togetherness $\quad$ When being together... through using the home language}

As soon as arriving at the centre, Xiaohan walked to two of her Chinese peers who were playing in the sandpit. (Xiaohan $/ 2^{\text {nd }}$ visit, 9:52am)

Leah called out to Fang, a Chinese peer the minute she saw Fang coming out of the sleep room. (Leah/ $1^{\text {st }}$ visit, $1: 45 \mathrm{pm})$

Eden saw his Chinese peer Joe on the top of the playhouse. Eden called Joe. (Eden $/ 1^{\text {st }}$ visit, 10:10am)

Amy moved to Ken, a Chinese boy and gave him a book.

(Amy/2 ${ }^{\text {nd }}$ visit, 11:21am)

Rick is holding a bike and calls loudly in the middle of the playground to his peer

Ben who is also in the playground.

(Rick/1 ${ }^{\text {st }}$ visit, 9:31am)

Jim walks to Wayne, a Chinese boy who is standing by a window watching outside. $\left(\mathrm{Jim} / 5^{\text {th }}\right.$ visit, $\left.1: 55 \mathrm{pm}\right)$
Xiaohan: "guo lai kan kan wo you shen me?" [Come to see what I got].

The peers are walking out of the sandpit to Xiaohan.

Ben, a boy calls on the way to Xiaohan: "shi shen me ya?" [What have you got?] .

Leah: "Fang, wo men dao wai bian hao ma?" [Fang, shall we go outside?].

Eden: "Joe, lai ya" [Joe, come here].

Amy: "Ken, xiang he wo kan zhe shu ma" [Do you want to read this book with me?].

Ken nods.

Rick: "Ben, guo lai ya. Wo men qi che!" [Ben, come here quickly. Let's bike].

Jim: "wo men qui wen shui hao ma?" [Shall we go to play with water?]

Wayne reaches his hand to Jim.
Xiaohan takes the peers inside and shows them a pink stone. The children examine the stone together before moving to the family corner as a group.

Fang walks to Leah and the two girls hold hands and walk outside.

Joe looks up and climbs to Eden.

Amy and Ken read the book.

Ben runs to Rick and takes the bike. Rick rides on a bike and Ben rides on the other. Ben follows Rick biking around.

Jim and Wayne play in the water tank. 
The behaviours in these examples illustrate the children's use of the Chinese language to initiate contact with peers, which led on to doing things together. The importance of using a meaningful language to develop togetherness with others was also noted by van Oers and Hännikäinen (2001). In the present study, the children seemed to be very aware of the usefulness of Chinese to connect them with Chinese-speaking peers. During their interviews, they gave the following reasons for their use of Chinese with Chinese peers:
Rick: $\quad$ We are all Chinese children
Jim: $\quad$ I can only speak Chinese
Eden: $\quad$ We don't like speaking English
Amy: $\quad$ Ken [a Chinese peer] does not know English
Xiaohan: I am a Chinese girl and they are Chinese too
Leah: $\quad$ Chinese people can speak Chinese. They [my Chinese peers] don't know English.

These comments confirm that being together with other Chinese children indeed serves as an important driving force for the study children to use Chinese. In other words, the children seemed to know that the Chinese language was a tool that could connect them with other Chinese speakers. At the heart of their use of the Chinese language with each other, therefore, is the children's awareness of the usefulness of the Chinese language to make meaning for them and other Chinese speakers, to have their needs understood, and to have them play together (Feng et al., 2004; Whitehead, 2007). In this sense, becoming a member of a group is likely to have been the reason for the children to use the Chinese language. Feng et al. particularly attribute ethnic minority children using their first language to their attempts to be together.

It should be noted too that these children's recognition of the effectiveness of Chinese language to build togetherness could also reflect their understanding of their common identity as Chinese, and of the way this identity grouped them together and made them distinct from many other people in their centres. It is at such a point that what appears as an observable feature of the children's attempts to be together could be the result of their desire for maintaining their "we-ness" and group uniqueness as Chinese 
(Hännikäinen \& van Oers, 1999, p.6). There is evidence from Pfeifer, Rubble, Bachman, Alvarez, Cameron and Fuligni (2007) that minority children in a multiple-group setting evaluated their own ethnic group positively, and were happy to belong to it. Moreover, if we use Wartofsky's (1979) notion of cultural modes of representation to understand the children's behaviours, one could argue that the children's attempts to form Chinese groups is a way for them to make visible the meanings of their family culture within non-Chinese cultural contexts. In doing this, some children, particularly Xiaohan and Jim who were newcomers in the centres, could be seeking "a distinctly human form of actions" with which they were familiar (Wartofsky, p.202) to support their way of being in an unfamiliar place. Through their experience in the centres, these children might have realised that they could not quickly communicate and interact with Englishspeaking peers. Therefore, they needed to develop relationships with the peers with whom they could speak as a way to gain security and a sense of belonging in the new learning setting.

All six study children who had Chinese peers were observed to engage in frequent interactions with specific Chinese peers. Although the study did not capture how the friendship with these children was established, I noted that these children tried hard to maintain the togetherness with their Chinese peers and the use of a common language clearly served this purpose. This is especially so for Leah (4:6/C) who seemed to watch how her social group with two other Chinese children was functioning and protected it from being dismantled. Although Leah spoke English well, she used Chinese exclusively with her Chinese peers and allowed no English in their conversations. The following example from my field notes illustrates how Leah conversed with her Chinese peers in order to maintain their togetherness.

Leah, Fang and Will are sitting on a bench outside, watching other children playing. Two girls in the near distance play a doll. The teachers and other children are elsewhere. Fang: "wo ye you yi ge bu wa wa" [I have a baby doll too].

Leah: "wo de hao da" [mine is big].

Will: "wo you yi get xiao wan ju gou" [I have a toy puppy].

Fang: "wo you yi ge zhen gou. Wo de go go xian zai zhe ma fat. He eats too much" [I have a real puppy. He is so fat. He eats too much], stretching her arms open to show the size of her dog.

Leah gives Will a glance, saying: "wo zhi dao ni de go chi hao dou. Will ni de go chi de duo ma?" [I know your dog eats too much. Will, does your dog eat too much too?]. (Leah/1 ${ }^{\text {st }}$ visit, 2:09pm) 
In this excerpt, Leah quickly translated Fang's English words to Chinese and she did this every time that Fang included English words into the conversations. Retaining the use of Chinese in the Chinese group was a recurring theme in Leah's data. As the oldest of the three Chinese children, Leah might have been trying to ensure that Will, who knew very little English, was included in the connection thereby "keeping member[s] within the group" (Paulston, 1992, p.36). What Leah did in this excerpt can be supported by her comment in the interview that "Chinese people can speak Chinese and my Chinese peers don't know English".

Xiaohan $(4: 8 / \mathrm{M})$ also appeared to monitor the use of the Chinese language to maintain social bonds when she quickly stopped peers from using English in the following excerpt.

Peter (a Chinese boy) is digging in the sandpit with Xiaohan and Rick. They stand close to each other. Two other children are digging on another side of the sandpit, but they do not interact with Peter, Xiaohan and Rick.

Peter hands the spade to Rick: "your turn. I'm tired".

Rick: "I am tired too".

Xiaohan drops her spade on the ground, holding Rick's hand: "zou, wo mean bu wan zhe ge le" [let's go to play other things].

Peter: "me too", putting down his spade.

Xiaohan: "wo men shou zhong guo ha" [let's talk in Chinese]. (Xiaohan/ $3^{\text {rd }}$ visit, $1: 09 \mathrm{pm})$

Unlike Leah who aimed to keep Will in the group, the motivation for Xiaohan's behaviour might have been to be together with the peers. Because of Xiaohan's limited English, the Chinese language helped her maintain membership with her Chinese peers. It is important to note here that when Xiaohan said 'let's talk in Chinese', she put emphasis on the word 'Chinese'. This word is important here because when using it, Xiaohan highlighted that their togetherness was due to the fact that, being Chinese, they could all speak Chinese. In this way, she made visible the influence of their shared identity on their use of Chinese and indicated that they were doing this together because of it. Xiaohan's behaviour again confirms the concept of 'we-ness' mentioned above. When children like Xiaohan and Leah worked to maintain group togetherness for themselves and their Chinese peers, they demonstrated a clear understanding of their own identity as Chinese as well as that of their Chinese peers, and that the Chinese language was a tool of togetherness within their ethnic group. 
In addition to their attempts to be with Chinese peers through using the Chinese language, several study children used it to contact Chinese adults and to maintain togetherness with them.

Xiaohan told me: "May (her Chinese teacher) is Chinese. She understands me". The field notes from Xiaohan gave many examples of her talking to May and being with May. I once observed her looking around when first arriving at the centre. She asked me: "where is May?". In that situation, Xiaohan told me that "I just want to talk to May and be with her".

In Jim's case with Ellie, a Chinese speaking teacher, Jim knew that Ellie could speak Chinese when he first joined the centre. Ellie told me that "I met Jim's mother on Jim's first day and had a talk with them in Chinese so Jim knew I could speak Chinese". It is clear that Ellie and Jim established their relationship through the Chinese language and maintained it likewise. This further supports the point from Baker and Jones (1998) that second language learners could easily make a language choice with the people they knew, as their relationship had usually been established with one language.

At Luke's centre, he was the only person who could speak Chinese. However, during the period of my data gathering, Luke made many attempts to speak Chinese with me and to stay around me. Luke initiated a conversation with me in Chinese when he first saw me at the centre, and after that he regularly attempted to talk to me. There is no doubt that our common language helped us connect.

The most interesting information from this study about the children's use of Chinese to build togetherness with me was from Leah. Although Leah spoke only English with Ellie, the Chinese teacher at her centre, she never spoke English with me, even though I looked similar to Ellie as a Chinese adult who spoke English with others. In response to my question about why she only used Chinese with me, Leah explained: "because it is OUR (voice up) language. We don't let others hear'. In this reply, she indicated that her use of the Chinese language with me was not simply to exchange messages, but was also for making a connection and a sense of 'we-ness'. Her word 'our' here might be synonymous with Xiaohan's use of the word 'Chinese', that grouped us together and also set us apart from others. Since 'our' is an inclusive pronoun, implicit in this word is 
a purpose of including me with her. In addition, because she shared with me "some secrets" (informal conversation with Leah), Leah might have felt safe to use a language that would have been understood by few people. Moreover, since Leah had met me at her home before my visit to her centre, she might have treated me more as a friend than a teacher like Ellie. In this way, besides building a sense of 'we-ness' with me, Leah was also drawing divisions between friends and non-friends (Scourfield et al., 2006). This again confirms the power of language to create unique meanings for those who use it thereby connecting the users of that language (Joseph, 2004).

\subsubsection{Expressing personal needs}

Beyond using the Chinese language with Chinese people to build a sense of togetherness, the study children also used it to express their needs. In doing so, the Chinese language, as a familiar cultural tool, specifically supported their practice in an unfamiliar cultural community.

Jim (3:1/M) used Chinese to ask Leah, a Chinese peer, for help. This reliance on Leah was in part because, as Jim's teachers told me in our interview: "Leah was allocated to help Jim when Jim first joined us". This arrangement allowed Leah to spend time with Jim, which Jim made good use of. The following example illustrates Jim's use of Chinese to seek help from Leah:

Jim is walking to Leah: "wo ne xie zi tuo ba xia le" [I cannot take off the shoes]. Leah: "wo Kankan" [let me look]. (Jim/2 ${ }^{\text {nd }}$ visit, 10:29am)

On nearly every occasion that Jim asked for help, Leah responded, which could have confirmed for Jim the value of using the first language with Leah to have his needs met. So Jim approached Leah for help.

Jim asked Chinese adults for help in Chinese too.

Jim is looking around for paper.

Jim comes to me, who is sitting at a table in the room: "wo xiang yao zhi" [I need paper].

Karen: "zhao yi zhao. Wo bu zhi zai na li" [take another look. I don't know where they are]. 
Jim keeps looking for a while, then walks outside to Ellie: "wo dao na qu zhao zhi ya?" [Where can I find paper?].

Ellie holds Jim's hand: "zhe li, gen wo lai” [here, come with me]. $\left(\mathrm{Jim} / 1^{\text {st }}\right.$ visit, 2:11pm)

On another occasion, Jim walked up to Ellie asking her about his drink bottle. Ellie was not physically close to Jim, but after he failed to find his bottle, Jim deliberately moved away from the teachers who were nearer to him to Ellie. On both occasions, Ellie helped Jim, thereby assuring him that, as he had done with Leah, using Chinese with Ellie would meet his needs.

The data also record Eden's (3:3/M) purposeful search for Dawn, and asking her for help. In those situations, Eden's behaviour was very similar to that shown by Jim. He walked to Dawn with a query, regardless of where Dawn was at that time.

It seems reasonable to explain these children's experiences similarly to that above that show Jim approaching Leah for help: The children understood the usefulness of Chinese to help them in a non-Chinese speaking setting when they spoke it with Chinese language speakers. The children's behaviours here can be summarised as resembling the learning experiences of other second language speaking children described in the research literature (Flores et al., 2002; Igoa, 1995; Weisman et al., 2007). The data discussed in this section illustrate the unique position of Chinese-speaking people to mediate the learning experiences of a new language and culture for the children who were at the early stage of learning them.

\subsubsection{Expressing needs with English speaking people}

In both Jim's (3:1/M) and Luke's $(3: 3 / \mathrm{M})$ data there was evidence that as with the children reported in Tabors's (1998) study, when a common language of communication did not exist, the first language was used to express needs with second language speaking people. For example, during one group time, I observed the following interaction:

Joy (teacher) sits on the floor with a group of children including Jim. Joy holds a box in her hand. She takes a card out and calls out a child's name. The child named stands up and picks it up. Joy takes more cards out and children whose names are called collect their cards. Jim sits on the floor appearing to be concentrating on what Joy is doing. He then stands up, walking to Joy: "na yi ge shi wo de" [which one is mine?]. Joy smiles to him and hands him a card. (Jim/ $3^{\text {rd }}$ visit, 11:33am) 
In an informal conversation with Joy that day, I asked her whether she understood what Jim had said. Joy said she could tell what Jim wanted because it was clear that Jim was after the card. It is not certain whether Joy's appropriate response triggered Jim's repeated use of Chinese with her in another situation:

In the sleep room, Jim stands up from his bed, asking Joy: "wo yao niaoniao" [I need to go to the toilet].

Joy puts a figure on her lip and speaks very quietly: “xu....quiet", pointing to Jim's bed. Jim watches Joy for two seconds and walks out.

Joy watches Jim as Jim goes to the toilet and smiles to him when Jim comes back to his bed. $\left(\mathrm{Jim} / 4^{\text {th }}\right.$ visit, 12:30pm)

It is likely that Joy did not understand Jim's intention. Jim responded with a short wait but walked out after seemingly realizing that Joy did not understand him. It could also be that Jim was so desperate to go to the toilet that he did not want to wait any longer. In any case, Jim's utterance clearly expressed a specific need which was not understood by the English-speaking teacher Joy. Jim could not articulate the reason for his use of Chinese with Joy but his mother believed that "Jim is expressive; when Ellie is not around, Jim inevitably approaches others to make requests, even if he knows that Chinese might not work". Given that Jim switched the languages when talking to Wayne (Chinese peer) and an English-speaking peer in the same situation (see table 6.3), it is possible that he indeed knew the differences of the two languages and what Jim did in this situation with Joy was his intention to express an urgent need that he could not convey in English. Considering this scenario from the perspective of Joy, an Englishspeaking teacher, without the ability to make meaning of Jim's expression, a strategy that Joy used to cope with her own lack of Chinese competence, was to trust Jim to do the right thing and to show him her intentions by actions when words were no use.

Luke's data also included examples of his use of Chinese to English-speaking teachers.

Luke walks around on his own. He walks to a wardrobe and stops there, watching it. There is a sign on the wardrobe that says 'staff only'. Luke opens the door, trying to reach a drum inside.

Luke: "gu" [drum], murmuring.

Nicole (his teacher) comes and removes his hand: no, Luke.

Luke says again: "gu. Wo yao gu" [drum, I want the drum].

Nicole smiles at him and leaves.

Luke stands where he is and watches Nicole as she leaves. (Luke/ $3^{\text {rd }}$ visit, 3:31pm) 
In the following excerpt, Luke used Chinese with Hannah, another English-speaking teacher.

Luke sits at a table with eight children and his teacher Hannah. Hannah is kneading dough in a bowl on the table. The children are watching her. Hannah stands up: "I will be back shortly". The children wait. Hannah is back with a pile of plates and hands one to each child. Luke gets one. He puts it in front of him as his peers do. Hannah then hands a piece of dough to five of the children and instructs them what do. Luke watches, then stands up, bending to Hannah

Luke: "gei wo yi ge" [give me one]. Hannah points to the chair which he was sitting on. Luke moves back to the chair. (Luke/ $4^{\text {th }}$ visit, 10:35am)

In both situations, it is clear that Luke could not resist expressing his needs or wishes. He appeared to be very interested in the drum in the first example, and when stopped from reaching it, he might have been too confused and disappointed to do anything. At the dough table with Hannah, Luke asked for his share after seeing his peers had some dough. The reason for Luke's use of Chinese with English-speaking people in these two situations might be similar to when Jim spoke Chinese with Joy: Luke wanted to communicate his wishes and needs irrespective of whether he had the appropriate language. A feasible explanation for Luke and Jim's behaviour is that they used their first language to cope with urgent demands which their minimal command of English could not achieve (Arnberg, 1987; Reyes, 2004). The children did not appear to be demonstrating this behaviour because "they have not yet discovered that there is a new language used in this new setting" as proposed by Tabors (1998, p.22). Rather, as confirmed by their parents, they knew that English was the language of the new environment. Furthermore, research has demonstrated that children as young as two years of age could differentiate language use for people and contexts (Lanza, 1997; Nicoladis, 1998; Quay, 1993). In view of this, it is possible to infer that these children had immediate requests but could not make them in the language that they were learning, therefore they used Chinese with the English-speaking people. In Luke's situation with Hannah, when Hannah pointed to the chair in response to Luke's Chinese request, Luke moved back to his chair, clearly demonstrating he knew that Chinese would not help him, so that he just waited to see what would happen.

These incidents also highlight some additional implications of the communication gaps between the study children and their English-speaking teachers. For example, the fact that the children were eager to put their messages across but the teachers were unable to 
understand them could have been interpreted by the children as their teachers' lack of response to them. This suggests that in such contexts, the study children are likely to experience dissatisfaction of personal needs. Viewing these examples from the perspectives of the teachers, it is likely that the teachers perceived themselves as constrained in their ability to be responsive, by the fact that their communication with the Chinese immigrant children was limited to English or non-verbal communication. The teachers' choice of quickly ceasing the communication with these children might mean that the teachers did not feel confident to respond to the children's verbalization because the teachers did not know how to do it in a way that the children understood. The implication here, especially in view of the examples that illustrated Chinesespeaking teachers' appropriate support of the children in needy situations, is that appropriate language was required for the dynamics of particular meaning-making situations.

\subsubsection{Mediating thinking and guiding behaviours}

In table 6.2, I included examples of some children's interchangeable use of English and Chinese during private contexts. In those events, the children alternated between their use of the languages. I suggested that the children were engaged in these speech events in an attempt to think about what they were doing, to practise their English, or to make language interactions. In fact, when analysing the data of the children, the pattern that I could see is that speaking Chinese when being alone is a feature in the data of all the study children. There were differences, however, in whether they only used Chinese or, as shown in section 6.1, whether they added certain English words. While Luke (3:3/M), Eden (3:3/M), Jim (3:1/M) and Xiaohan (4:8/M) spoke mainly in Chinese in their private speech and Sarah (3:8/B) included English on a few occasions, Rick (4:5/AC) Leah $(4: 6 / C)$ and Amy (3:6/R) used both Chinese and English in many of their private talks.

As pointed out in section 6.1, Vygotsky argued that private speech helps children connect thoughts with words. In the data in Table 6.4, it is apparent that the children talked to themselves in Chinese to think about what they were doing. 
Table 6.4: Children's private speech in Chinese

Context

Jim is drawing at a table. Alongside him are two other children writing on paper. There are no teachers close by. $\left(\mathrm{Jim} / 1^{\text {st }}\right.$ visit, $\left.2: 15 \mathrm{pm}\right)$

Leah is pencilling a picture at a table on her own. Other children are playing elsewhere. (Leah/4 ${ }^{\text {th }}$ visit, 11:45am)

Rick walks around in the room. He picks up a plastic dinosaur on the floor. Rick holds it in his hand.

(Rick/ $2^{\text {nd }}$ visit, 9:48am)

\section{Data of private speech}

Jim murmurs: "wo hua yi ge da hu die, hu fei fei. Yi ge da hong hu die" [I am drawing a butterfly, one that can fly. It is big and red].

Leah: "oh, good. This is my fang jian" [my room] "This is wai po de fang" [grandma's room] "Mama de fang zai zhe" [mum's room is here].

Rick: "wo xu yao another dinosaur, xiang picture shang de nang yang" [I need another dinosaur, like the one on the picture book].
Thinking of how to develop the picture

Imagining the need for a dinosaur that is bigger than what he has got.

These examples throw light on what the children were thinking when they were speaking to themselves, thereby reflecting Vygotsky's (1978) hypothesis that children think about their actions when speaking to themselves. The use of the Chinese language provides evidence of the power of that language to mediate children's thinking systems. It suggests that the children had mastered the Chinese language and were able to use it to mediate thinking, irrespective of their English language competency.

Perhaps the reason for the children to use either Chinese or both languages in their private speech is the varied extent to which they had internalised the English language. Because Jim was at an early stage of acquiring English his ability to process information in English was limited. In comparison, Leah and Rick had some competence with English so that they might, to a certain extent, process thoughts in English.

Regulation of behaviours was another theme that emerged from the children's data of 'private Chinese speech'. This phenomenon illustrates the other function of private speech as a mediator for learning, as proposed by Vygotsky. In her research on children's private speech, Clark (2004, p. 100) observed: “Children serve as their own 
monitors of learning and behaviour by commenting on [their] thoughts and actions". In the example that follows, Luke $(3: 3 / \mathrm{M})$ was observed using private speech to remind himself of how to behave:

Luke is standing by a table, watching three children playing cards. He then turns around: "bu kan le. Qu niao niao" [don't watch here anymore. Should go to toilet]. Luke runs to the toilet. (Luke/2 ${ }^{\text {nd }}$ visit, 2:32pm)

During the data gathering phase of the study, Luke was undergoing toilet training. Luke's father reminded him to go to the toilet every morning that he brought Luke to the centre. In making this comment, Luke was monitoring and guiding his behaviours.

A similar observation was recorded for Eden (3:3/M).

Eden is running outside. He stops at the door and says: "oh, wang dai mao zi le" [oh, forgot the hat]. He then turns to his bag, finding the hat, putting it on and going outside again. (Eden $/ 1^{\text {st }}$ visit, $\left.1: 56 \mathrm{pm}\right)$

In this episode, Eden clearly told himself to find his hat before going outside.

Using language as a tool to mediate behaviours indicates an important point in relation to the children's learning experiences: language is a useful tool to impose order and control to them; the children, such as Luke and Eden, began to experience being part of the centre. Through the Chinese language, the language they could use well, the children were learning appropriate actions in the new cultural community. In this view, the Chinese language was indeed an important tool that guided the children's transformation of cultural practice (Rogoff, 2003). Through speaking Chinese, even in private speech, the children gained important knowledge about their new cultural setting.

Following the analysis in these sections, one can see that the children's use of the Chinese language in the centre acted as a mediator for the children's establishment of a sense of belonging there. It also helped the children express their needs and guided their thinking and behaviours. In this way the cultural tool of the Chinese immigrant family (language) provided the children with a bridge through which they moved from one culture to the other, as shown in figure 6.2. This figure adds to figures 5.1 and 6.1 that illustrated the convergence of the two cultures, by providing another possibility for 
intercultural relationship between the Chinese immigrant family culture and the mainstream culture of New Zealand EC centres.

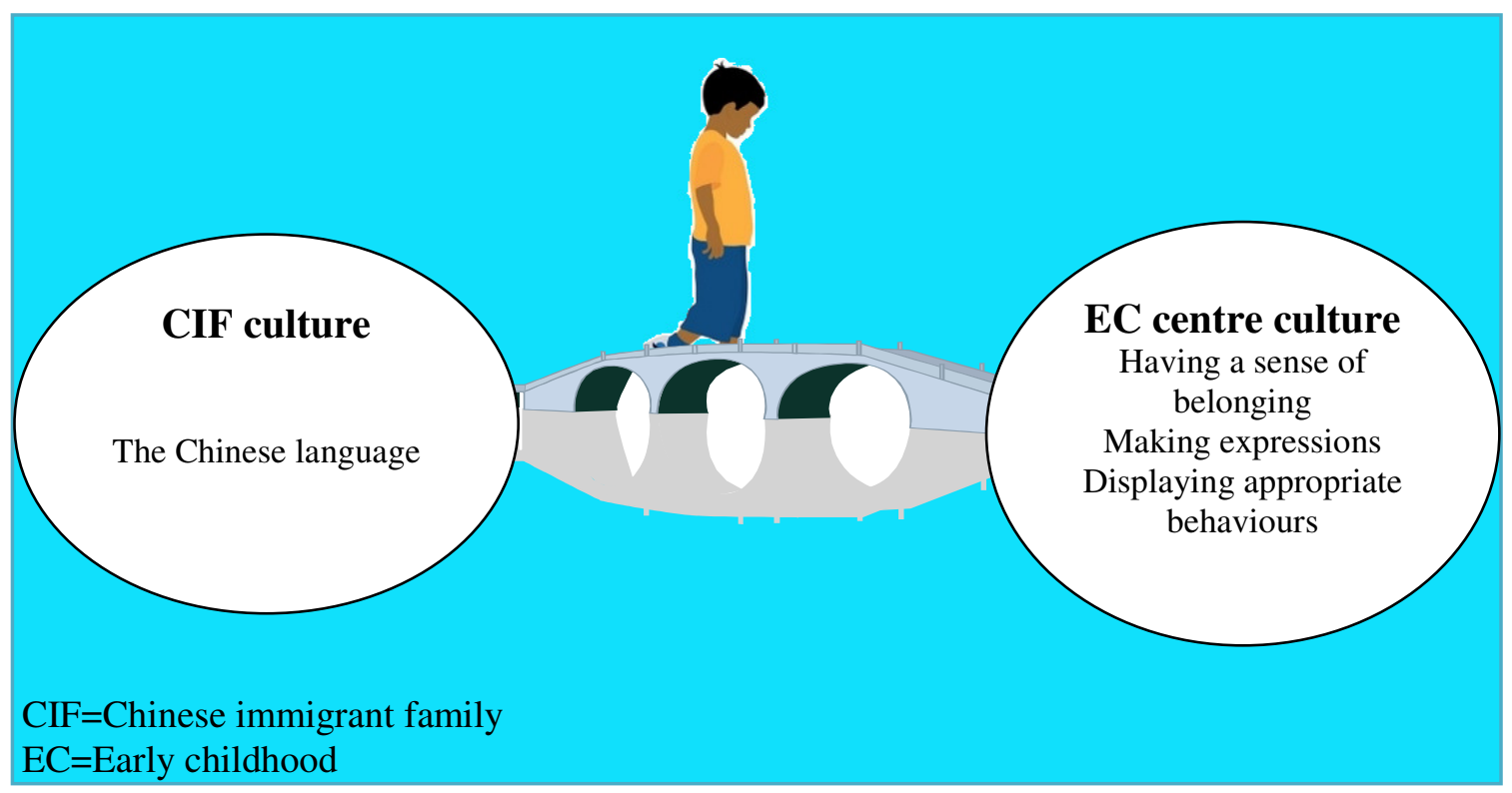

Figure 6.2: The Chinese language bridges the two cultures

\subsection{Experiencing the English language}

\subsubsection{Working towards bilingualism}

The data on children's use of English indicate that being aware of the importance of using English or learning to use English was a recurring theme. This is reflected by some children's enthusiastic communication in English with English-speaking people and their adoption of a number of strategies to learn or to use the English language.

\subsubsection{Communication with English-speaking people as a way of learning the} language

Some children, namely Leah (4:6/C), Amy (3:6/R) and Rick (4:5/AC) appeared immersed in developing their English language and they saw the EC centre as the context for this. They participated in many interactions in English, and on various occasions, Leah initiated as well as responded in English with her English-speaking peers and teachers. Rick used English actively when required, and Amy, although quieter than Leah and Rick, appeared to create and take opportunities to communicate in 
English as well. The other five children, Xiaohan (4:8/M), Jim (3:1/M), Eden (3:3/M), Sarah (3:8/B) and Luke (3:3/M) rarely spoke English. However, they appeared to have adopted other strategies to communicate in English which will be discussed in section 6.3.1.2. These children's actions to actively communicate, or to learn to communicate in English indicated their recognition that English was an essential part of their lives in the centres and that it was important that they spoke in English as a way of learning it.

Children's use of a language is believed to be related to their proficiency with it (Paulston \& Tucker, 2003). This suggests that the variations in the use of English among the study children could possibly be an indicator of their competence with that language and their awareness of their competence. Leah had the highest standard of English, Rick was able to communicate in many contexts and Amy could use it for routine conversations. The children who spoke little English, were described by their parents or teachers as not having established the ability to use English yet. Therefore, the active use of English by Leah, Rick and Amy to some extent mirrored their confidence with it. A logical conclusion then is that the children who spoke little English had less confidence with it.

Leah's active use of English can be seen as a result of her intention to develop competence with it. This is supported by her interview data, in particular, her statement that, "I should learn English". Even though both her teachers and I thought Leah spoke English well, she was not too satisfied with her proficiency and it is clear that Leah wanted to improve further. In addition, Leah's data reports numerous incidents of her playing and enjoying socialising with her English-speaking peers and teachers. These data indicate that Leah's active use of the English language could also be attributed to her desire for social integration. English was useful to her, thus she was motivated to learn it through actively using it. Lambert (1973) contends that self motivation is a powerful driving force for one's development of a new language. Analysis of Leah's data using Lambert's motivational theory suggests that the usage of the English language was grounded in Leah's own needs and interests. Like the learners in Spolsky's (1969) discussion, Leah appeared to have developed a very positive attitude towards English and English-language speakers. Spolsky wrote that "one of the most important ...factors is the attitude of the learner to the language and to its speakers" (p.274). Further evidence that helps understand Leah's active use of English came from 
her mother and her teacher Carol. From their point of view, "Leah love[s] talking. She made friends in this way" (Leah's mother) and "Leah enjoys communication because this helps her become popular" (Leah's teacher Carol). These opinions imply that Leah's active communication in English was both dispositional and motivational. This combination of motivation to gain peer popularity and to develop the new language could, therefore, explain Leah's engagement with the English language. To put it simply, Leah had a habit of communicating with people and had learned about the value of doing this. In an environment where English was the predominant language, she had no doubt worked towards learning and using the language.

In addition, it is important to recognise that Leah's behaviour with the English language could be dependent on other contextual and historical factors. She was five and had been in the centre for over two years. When newcomers are in the situation of being exposed to a new language, they are inducted into the particular contextual practices and over time develop skills and competence to participate in communication events (Shi, 2006). If one accepts that learning takes place through participating in communities of practice (Wenger, 1998), then Leah's two-year process of becoming a member of her EC centre would have helped her learn to function in an English-speaking learning environment.

Rick's data included several situations in which he was actively involved in communication events in English, as illustrated in the following example:

Rick walks onto the edge of the sandpit. Rachel and Iris, two teachers are talking in the sandpit. Two other children are playing around them.

Rachel: "my foot got hurt the other day".

An English speaking child turns to Rachel: "Did you bleed?"

Rachel: "yes, I got a cut".

Rick gets down to Rachel and watches her: "did you cry?"

Rachel: "I cried because my feet hurt".

Iris: "it is okay to cry. I cry when I watch a sad movie".

Rick: "my brother cry. I don't".

Rick then picks up a stick and moves to the sandpit, drawing a round circle in the sand. Rachel moves close to him: "Rick, what are you drawing?"

Rick: "a crying face". (Rick/2 ${ }^{\text {nd }}$ visit, 3:52pm)

Rick entered an existing conversation and engaged himself in the context. Although the utterances he made were short, Rick took language turns naturally, appeared confident to talk, and endeavoured to follow the theme of the conversation. He not only used 
words but drawing too. I observed Rick talking to Rachel for 11 minutes. After drawing the circle, Rick drew tears on the face and told Rachel that his brother had tears on his face one day and he washed them off for him. Rick's behaviours in this scenario show a clear theme: he initiated his own involvement in an English-communication opportunity. This is an example of a child sharing feelings with his teachers in a situation in which they had joint interest and engagement in conversations and play. It is very likely that Rick wanted to belong and to have interactions. These conversations are premised on reciprocal structures associated with the experiences of the child and his teachers. The conversations were sustained between Rick and his teachers because the teachers tuned in to Rick's language, comprehension and experience levels. So Rick was able to talk with his teachers. In addition, given that learning a new language is a process of 'living in' the language environment, Rick's exposure and the length of time in the EC centre, could also have helped develop his communication in English, in the same way as in Leah's case described above.

An interesting behaviour in Amy's data is that she talked mainly to the teachers. As discussed in Chapter 5, Amy closely followed teacher instructions for her learning, because she was aware of the importance of a teacher's role being to teach. Amy's interview data similarly suggested that her active use of the English language with teachers was motivated by her desire to "learn English with teachers" (Amy's interview). The following example occurred in Amy's data:

Amy walks to her teacher Fiona (sitting on the floor by the window, reading a book to a child), pointing to her hair clip.

Fiona: "oh, that's pretty" (raising her voice).

Amy: "my sister got pink... me no like pink".

Fiona: "what colour do you like, red?", pointing to Amy's hair clip.

Amy: "and green".

Fiona: "that's good. I also like green". (Amy/2 ${ }^{\text {nd }}$ visit, 9:13am)

This short interaction shows the typically active way of initiating exchanges which Amy displayed with a teacher. In contrast, as will be discussed in Chapter 7, Amy's communication experiences with English-speaking peers were very limited, perhaps because of the difficulties Amy encountered when trying to communicate effectively with English-speaking peers. Amy might have been aware that in order to fulfil her commitment to staying in the centre (see section 5.4.3), and her expectation of learning 
English, she needed to interact with the teachers. It is also very likely that Amy felt comfortable with her teachers because the teachers provided her with focused attention (see section 5.2.1).

A Chinese cultural orientation of focusing on learning could have influenced these children's attitudes to learning the English language. Evidence of this came from the interview data of Leah and Amy's parents. According to Leah's mother, traditional Chinese stories had always been read to Leah, so her interest in learning might have developed from her home experiences. Amy's parents told me that they purchased many Chinese cartoon programmes for Amy and her sister to watch. The parents sometimes used Chinese stories to show Amy the importance of working hard. Because these children were trained at home to work towards their goals, it is reasonable to expect that they would find ways to learn English, if they considered English to be important.

\subsubsection{Using strategies to communicate and to learn}

Research has found that young children who have acquired a first language are able to learn and use a new language with strategies such as silence, imitating, repeating and body language (Konishi, 2007; Liu, 2006; Siraj-Blatchford \& Clark, 2001). The use of these strategies is a process of associating thoughts with actions to learn, understand and retain (Rivera-Mills \& Plonsky, 2007). These approaches stem from 'the learners' innate self-regulatory capacity that fuels their effort to search for and then apply personalised strategic learning mechanisms" (Tseng, Dörnyei \& Schmitt, 2006, p.79). The strategies are described as "goal directed, intentionally invoked and effortful" (p.80).

Like children in other studies, the children in this study appeared to have also developed a belief in the effectiveness of strategic behaviours to help them learn or to use the English language. Apart from the active communication with English-speaking people of three of the study children, as discussed in the above section, all the children's data suggest strategies that include the use of body language; application of single words or formulaic speech; imitation; seeking language help; practising; making a new language; and silence. Each of these strategies is discussed in the following sub-sections. 


\subsection{Body language}

Most typically, the children's non-verbal communications included: nodding, shaking their heads, pointing, smiling or passing things to people.

Body language was used by some children for making requests. Jim (3:1/M) gave his non-Chinese speaking teacher, Helen, a nudge and then pointed to a door. Helen then took him through that door to a room, where Jim stayed constructing structures with blocks. Before interacting with Helen, Jim had gone there but could not get in because the door was shut. Clearly he was using body language to make a request to Helen. Similarly, Luke (3:3/M) used body language when he asked his teacher Jess to open his packet of chips and when he wanted a pen like the one held by another child. Body language was also commonly used by the children when they were making requests to English-speaking peers. Body language was effective in most situations, and in Luke and Jim's cases, it enabled them to get what they wanted.

The children also used body language to respond. Xiaohan (4:8/M) shook her head quite often when spoken to and she told me on one occasion, after she did this with her teacher Jenny, that it was because "I don't know what she was talking about". The interaction I had observed was as follows:

Xiaohan is building blocks on her own. Jenny walks over.

Jenny: "I remember you made a big building yesterday. Are you making another one?" Xiaohan turns to Jenny and stares at her. Xiaohan shakes head.

Jenny: "yes?, make another building?"

Xiaohan stares at Jenny for 6 seconds. She then turns back to the blocks from Jenny and continues with her building.

Jenny leaves. (Xiaohan $/ 5^{\text {th }}$ visit, 2:22pm)

Xiaohan's body language conveyed her lack of comprehension. By staring at Jenny, shaking her head and then shifting attention from Jenny, Xiaohan should have revealed to Jenny her confusion about what Jenny had said so Jenny left.

In addition, the children, including $\operatorname{Jim}(3: 1 / \mathrm{M})$ and Eden (3:3/M), whose English was minimal, responded to English messages using body actions. For example, Eden jumped off a chair and went to hold the parachute with peers when his teacher Kathy, having seen him sitting on a chair watching asked: “do you want to join?" Jim handed a piece 
of paper to a child during one group time when Helen asked: "Jim, will you give Matthew the paper?" It is clear that the children understood the situation and responded accordingly. Although it was not certain whether they would have been able to verbally respond, the use of their bodies to respond in these cases was appropriate.

Body language was further used for communication to support verbal language. Leah (4:6/C) and Rick (4:5/AC) incorporated smiling, laughing, moving around and pointing during their conversations with their peers in English-speaking contexts. Some children who could not speak English well were noted to use their bodies to convey messages when trying to interact with peers and teachers. Most often, they gave objects to them. For instance, Jim handed a pen to a peer when seeing the peer coming to the drawing easel where Jim was working. Luke (3:3/M) was also observed handing his cookies to a peer at tea time. Without accompanying this with speech, Xiaohan $(4: 8 / \mathrm{M})$ walked to her teacher Iris and handed her a picture that she had drawn.

In the later part of this chapter and in Chapter 7, there will be further examples about the children's use of non-linguistic ways of communication, including for example, their 'watching', 'glaring', 'staring', or 'smiling' at the English-speaking people they interacted with. I interpreted these behaviours of the children to have reflected clear sociocultural functions of the language because the children were reading the cues of the people as well as attempting to convey their expressions, showing "language use as a cultural practice, with specific rules and tools" (van Oers, 2007, p.301). The use of body language by the study children, thus illustrates that their communication was characterised by strategic engagement and attention to useful means.

\subsection{Single words or formulaic speech}

Communication in single words or formulaic speech was a strategy used by the Chinese immigrant children who had limited English competence, although the children who could communicate well in English also used it. In this section, communication in single words is interpreted as the use of one word to represent a whole speech act and formulaic speech is the use of a number of speech formulas or ready-made expressions (Ellis, 2002). 
In discussing the use of single words or formulaic speech as a communication strategy, I only refer to the data from children who had limited English competence, because they were more likely than the others to use these as a strategy to handle English-speaking situations. Table 6.5 displays some examples of the children's use of single words or formulaic speech.

Table 6.5: The use of single words or formulaic speech

\begin{tabular}{ll}
\hline Data & $\begin{array}{l}\text { Single words } \\
\text { /formulaic speec }\end{array}$ \\
Jim stops in the middle of his & 'Toilet' to his \\
movement with the peers and his & teacher Joy \\
teacher Joy. He walks to the door. & \\
Joy goes to him: "Jim, going out?" \\
Jim: "toilet", pointing to the toilet. \\
$\left(3{ }^{\text {rd }}\right.$ visit, 12:06pm)
\end{tabular}

Luke is standing by two boys who are building blocks, and watching them. Luke picks up a block from the floor and hands it to a boy:

"here".

The boy does not take it.

Luke raises voice: "here, give you".

The boy: "put it here", pointing to a space by him. ( $2^{\text {nd }}$ visit, $\left.11: 25 \mathrm{am}\right)$ 'here' to a peer 'give you' to a peer

\section{Interpretation of children's communicative intent}

Jim used the word 'toilet', a single word that contains the meaning of a full sentence to tell Joy what he wanted. He knew that word and used it appropriately. Jim also accompanied the word with his body language, 'pointing'. He appeared to have tried to make himself clear to Joy.

Luke showed a desire to get the boys' attention. He might have realised that accompanying the 'handing' action with words was more likely to pass the message to the boys. Following his failure to gain the boys' attention, Luke used a formulaic speech 'give you' to reinforce his aim. Although the boy did not take the block after Luke's second attempt to speak, he made a response. In this case, Luke repeatedly used words probably because he knew words could help him achieve the aim.

Xiaohan used body language and a formulaic phrase to express herself.

\footnotetext{
Xiaohan makes 'an ice-cream' out 'for you' to a peer of sand. She leaves the sandpit, goes to a girl and hands her the 'ice-cream': "for you". ( $2^{\text {nd }}$ visit, 11:34am)
}

There are examples in the data from all the children of their use of single words or formulaic speech. This phenomenon may support Weinert's (1995) view that single words and formulaic speech are a popular way of communicating among young second language learners, because they are short and easy to use. In these examples, the use of 
single words or formulaic phrases accompanied by specific body language served the purpose of making the children's messages understood. This also illustrates that when they knew they could do it effectively, most children attempted to speak in English. Therefore, using English, or learning it, was important for the children.

\subsection{Imitation}

Another important strategy used by the children and supported by research (PataryChing et al., 2006) is imitation. This was identified in the data as taking a word or words modelled by another speaker and repeating them, a definition based on Chesterfield and Chesterfield (1985).

All the children, including Eden (3:3/M), who showed very few attempts to use the English language, imitated what other people said when they needed to communicate in English. In the scenario below, Eden was playing cards with Sue, his teacher and some peers. After a round, Eden answered Sue in the following way:

Sue: 'Eden, want to play again?'

Eden watches Sue's eyes: "want to play again".

He then repeats: "play again".

Sue: "good", handing to Eden some cards. (Eden/3" visit, 10:26am)

Although it is not clear whether Eden created a response or was simply copying Sue, he clearly repeated what Sue had said when it was his turn to respond. Similarly, when he was screen printing with Sue and some peers, Eden said "I did it", following a peer who had said "I did it". So, his examples illustrate that when needing to take part in a learning experience, Eden paid attention to how other people spoke and adopted imitation as a strategy to function in the situation.

Sarah (3:8/B) listened to and copied her teacher Anne:

Sarah is painting at an easel with another child paining beside her.

Anne walks to her and asks: "what colour is this glitter?", pointing to the glitter on Sarah's picture.

Sarah puts the brush down and watches Anne.

Anne: "purple?"

Sarah: "purple".

Anne: "you got purple because you mixed green and blue glitters together".

Sarah: "mixed"

Anne: "they are shiny"

Sarah: "shiny". (Sarah/1" visit, 9:56am) 
Sarah did not answer the first question but she actively listened to Anne by putting her brush down in response to Anne's approach and question. Following that, the words 'purple', 'mixed' and 'shiny' given by Anne, were repeated.

Amy (3:6/R) too repeated her teacher Maria's words.

Amy is standing on a chair by the window when the teacher Maria goes to her.

Maria: "you be careful".

Amy gives Maria a glance.

After Maria leaves, Amy: "you be careful" and again "you be careful". (Amy/4 $4^{\text {th }}$ visit, $2: 54 \mathrm{pm})$

An interesting behaviour was observed with $\operatorname{Jim}(3: 1 / \mathrm{M})$ :

Jim is having lunch with peers, all seated at a table. Helen, his teacher is handing out bread.

"One more", the child next to Jim says to Helen.

Helen hands him a piece of bread.

"One more", Jim copies.

As soon as he gets the bread, Jim stands up, putting it into the rubbish bin. $\left(\mathrm{Jim} / 2^{\text {nd }}\right.$ visit, $12: 10 \mathrm{pm})$

Jim might not have been absolutely sure of the meaning of the words, or he might have said them just for fun, but he did listen and copy the words, thereby possibly displaying a learning behaviour. In addition, Jim could have been seeking the teacher's attention. When he saw that Helen, his teacher, attended to a child when the child said 'one more', Jim copied this, although the fact that he immediately put the bread in the rubbish bin suggests that he was not asking for the bread. It is also possible that Jim wanted the bread initially, so he copied the peer because the peer got it, but Jim changed his mind after getting his bread.

Xiaohan (4:8/M) called an English speaking child "Christina" and told me afterwards that that she did this "because I heard people calling her that name". On another occasion, she used "come on" with a child because "other people used that" (Xiaohan). Although evidence was not collected about Xiaohan's explicit imitation of words, she might have been doing what Ellis (1985) explained as an outer representation of memorization; that is, she could have memorised how people used the new language and imitated them in an inner way. Unlike the other children who articulated their imitations, Xiaohan might have felt more comfortable doing it inwardly. Even so, these children's behaviours had a common purpose of communicating in English. 
Vygotsky's (1987) point associated with the use of imitation is that it is a cultural practice. The above scenarios showing instances of imitation are evidence of the children's practice of "stepping from something [they] know to something new" (p.187). Hoel (1999) confirms that imitation is a crucial aspect of children's development within their ZPD because imitation helps children internalise a new function in their cultural communities and it is a means of joining others in cultural practices. Clearly, for both Vygotsky and Hoel, imitation is a specific mode of learning, with which the children in this research were indicating that they were being strategic with their learning of a new language.

\subsection{Asking Chinese speakers to help}

Jim and Eden asked their Chinese peers or adults to help them understand English.

Table 6.6: Asking Chinese speakers to help with English

\section{Data}

$\operatorname{Jim}(3: 1 / \mathrm{M})$ is digging in the sandpit and Joy (English teacher) is calling him: "Jim, where is your bottle?"

Jim stands up, looking puzzled.

Jim walks to Leah, who is sitting on a seesaw with a peer about ten metres away from the sandpit. Jim asks loudly, "Leah, bottle shi shen ma?" [Leah, what's bottle?]

( $2^{\text {nd }}$ visit, $\left.2: 15 \mathrm{pm}\right)$

Eden $(3: 3 / \mathrm{M})$ is eating at a table. His teacher Kathy is handing out apples to the children. Eden reaches a hand to the plate that Kathy is holding.

Kathy: "Eden, finish what you've got first. I'll save one for you".

Eden stares at Kathy. He then leaves the chair, goes to Dawn, who is sitting at another table.

Eden asks Dawn: "ta gao su wo shen ma?" [What did she tell me?] $\left(1^{\text {st }}\right.$ visit, $\left.12: 08 \mathrm{pm}\right)$

\section{Chinese speakers The child's intention involved}

Leah, a Chinese peer

To understand what Joy said to him

Dawn, a Chinese speaking teacher.

To understand what Kathy told him. 
Asking for help is identified by Krupa-Kwiatkowski (1998) as a "mediation strategy" (p.143), meaning that the people offering help mediate the learning situations for the learner, which agrees with Vygotsky's concept of mediation. By asking a Chinese speaker to help with their understanding of English, these children purposefully sought a mediator for their understanding of the communication contexts.

\subsection{Practising English with the Chinese peers who cannot speak English}

In section 6.1, there is a scenario of Amy (3:6/R) speaking English with her younger Chinese peer Ken. Xiaohan's (4:8/M) data also included her using English with a younger, less experienced Chinese peer, Red. This section presents one other example from these two children's use of English with Chinese peers and explains them as strategic practices for learning English.

Xiaohan watches outside in the window. She sees Red and goes to Red: "let's go, Red", holding Red's hand. (Xiaohan $/ 3^{\text {rd }}$ visit, 11:55am)

Red was only 2 years old and knew very little English. This incident occurred after Xiaohan had been called by her teacher in a similar manner to move to a group activity. Xiaohan might have picked up the phrase there and practised on Red.

Amy was doing exactly the same thing with her Chinese peer Ken, who was also much younger than her and had little English.

Amy and Ken sit on the floor, playing animals.

Amy hands a toy lion to Ken.

Amy: "shi zi" [lion].

Ken watches her.

Amy holds Ken's hand and they go to the animal box. She takes out a 'rat'.

Amy: "rat".

Ken makes no reply.

Amy picks up a crocodile: "crocodile".

Ken: "no", raising his voice.

Amy raises her voice too: "yes, crocodile".

Amy: "look, dog, dog", pointing to a toy dog on the floor. (Amy $/ 3^{\text {rd }}$ visit, 11:06am)

Ken did not seem to have understood the English words Amy used with him. With reference to Siraj-Blatchford and Clark's (2001) idea about the importance of a stressfree environment for second-language learners to practise the new language, it might be that Xiaohan and Amy did not intend to engage their peers in dialogues but simply practised the new language with them. Due to Red and Ken's limited English language 
proficiency, Xiaohan and Amy might have known that these peers could not judge them, so that they could practise the new language with them in a stress-free way. Choosing these younger peers as the safe context, therefore, was a strategy that Amy and Xiaohan adopted to communicate and learn the new language. This point fits with the finding of Krupa-Kwiatkowski (1998) who showed a second-language speaking child using the new language more frequently and confidently with the peers who were similarly learning the new language. She also understood it to be due to the child's intention for non-judgmental practice with the new language.

\subsection{Inventing a new language}

Jim $(3: 1 / \mathrm{M})$ and Luke $(3: 3 / \mathrm{M})$ uttered words that were neither Chinese nor English. They appeared to have produced an "invented language" (Krupa-Kwiatkowski, 1998, p.168).

Here is an example of invented language by Luke in response to his teacher Nicole.

Luke, all the other children and his teachers are inside. Luke is stands by the entrance door, watching outside.

Luke opens the door and steps outside. His teacher Nicole sees this and calls: "No, Luke".

Luke stops at the door, turns to Nicole, watching her, shouting: "waaameihai...."

Luke comes back to the room. (Luke/2 ${ }^{\text {nd }}$ visit, 9:10am)

Clearly, Luke was not using a language but making sounds. Carefully examining the sounds he made, I could see that they resembled the Chinese words 'wo hai mei' [I do not yet]. Probably, Luke intended to use the Chinese word 'I do not want', which suggests that, at that point, he wanted to challenge Nicole through the language that he could use. He did not use the correct Chinese language, possibly because Luke knew that Nicole did not understand Chinese, so it would not matter how he said that. Or maybe Luke started to assert himself in the Chinese language but then he changed his mind thus modifying the language. Krupa-Kwiatkowski (1998) tells us that beneath the surface of second language children's invention of a new language is their attempt to gain pleasure from playing with the language. It is therefore possible that Luke was taking delight in inventing a phrase.

Luke could have also known the power of his teachers to regulate his behaviours, and the consequences he would face if he challenged them (see section 5.4.2), therefore, 
instead of running away, Luke used an interesting technique of resistance that made sense to no one except possibly to himself. At first glance, this might appear to be a second-language speaking child verbally teasing his teacher, but when analysing it further, one can see that this inventive strategy may not be limited to an utterance of sounds; it might have served as an instrument of power too. Nevertheless, given that Luke reverted to compliance by turning the initial resisting phrase into an invented sound, for that part, Luke was likely to have revealed a perception concerning the importance of teacher instructions. It seems that Luke invented a phrase to help him overcome his initial urge to resist his teacher's power. Here the language again served as a mediating device that assisted Luke to regulate his behaviour.

Data on Jim's experience also included elements of 'invented language':

The tidy-up song starts for children to move to tidy-up time. Jim quickly leaves his blocks on the floor and jumps to the music.

He goes to the door, shouting to outside: "tadaadptai...", at the top of his voice. (Jim/ $1^{\text {st }}$ visit, 11:00am)

Jim continued making these sounds as he was helping tidy up. More than simply playing, it seems that he picked up the phonetic features of the words 'tidy up' because his sounds bore a clear resemblance to them. This suggests that the child had paid attention to the phonetic units of the words and was reproducing them (Baker, 2006). Underpinning the creation of this utterance, thus, was Jim's endeavour to learn.

These self-invented phrases of Luke and Jim are an example of the productive effects of language on children's learning experiences, and of the children's ability to express themselves in a creative way. The children did not depend solely on others to provide them with a sense of belonging, but engaged in a strategy that helped them to act as a member of a new language and cultural community. By taking unique ways to exert power, redirect social behaviour (in Luke's case), and to enjoy learning (in the cases of both Jim and Luke), the children contributed creatively to their own development as a member of the group.

\subsection{Silence as a learning and communication strategy}

Silence is described as a learning strategy and an internal engagement with knowledge and information in the new language (Baker, 2006; Granger, 2004; Siraj-Blatchford \& 
Clark, 2001). From a sociocultural perspective, it could be said that silence is a phenomenon that manifests a process of internalization in which learners transfer external knowledge into internal dimensions (Daniels, 2001).

While all the children in this study produced English utterances, some verbalised at a minimal level. Examples of this were collected from Xiaohan (4:8/M), Jim (3:1/M), Luke (3:3/M), Sarah (3:8/B) and Eden (3:3/M). My initial response to these children's apparent lack of speech was that they were not engaging with the English language. However, on closer scrutiny of their data, with particular reference to the information about their levels of English proficiency, it is likely that the children's silent behaviours indicated one of at least three things: (i) they were modifying their communication behaviours and holding back from speaking to avoid the embarrassment of making mistakes; (ii) they were internally learning the English language; or (iii) they had limited usage of the English language.

This section focuses on the first two possible explanations for the children's silence, and the section that follows discusses the data concerning some children's limited use of English.

While the learning aspect of silence has been well recognised (Granger, 2004; KrupaKwiatkowski, 1998), in this study, it is also seen as a strategy to avoid using English and as "a face-saving strategy in communication" (Jaworski \& Stephens, 2007, p.61).

The children and their parents' explanations of the children's lack of English speech, was that it helped them to avoid mistakes and thus saved face. Xiaohan told me "I don't know how to speak English". Jim also said: "I don't know English", while Sarah told me: "My English is not good". In a similar vein Sarah's mother said: "Sarah speaks English with her sister at home but not outside because she knows her English is not good. She could be afraid of losing face". Luke's mother also believed that the reason her son was so quiet in his EC centre could be his concern about mistakes, because he did not want to face embarrassment.

Putting this evidence together, it is likely that the children did not speak because they knew they had difficulties with English, were afraid of making mistakes and did not 
want to lose face. This finding fits perfectly with the discovery of Reeves (2005) whose study on young learners' experiences with a second language similarly demonstrated silent behaviours because these learners knew "if I said something wrong, I was afraid" (p.72). Reeves argued that the silence resulted from the children's psychological and cultural barriers so that the children "prefer the safety of silence to the danger of speaking" (p.72). Krupa-Kwiatkowski (1998) supports this point, saying "language that is eventually used with the target language speakers...is first arduously practiced in safer, less threatening environments" (p.171). Being silent, by this analysis, is a purposeful action aligned with the Chinese culture to build a respectful social image (Chang \& Holt, 1994). It is also a form of communication strategy because it required the children to modify their behaviours (Firth \& Wagner, 2007). It is interesting to see that the children at such a young age had behaved in line with the Chinese tradition of gaining social respect by trying to maintain their 'face'.

The internal and quiet way of experiencing the new language was also discussed by Siraj-Blatchford and Clark (2001) as a strategy for language learning. Following the data of these children, I could see that although the children did not talk much, they used many other strategies such as body language, self-invented language, single words or even memorization as illustrated in the above sections. These indicators tell us that when being quiet, the children could also be learning to "know how" to use the new language. Their silence, therefore, had learning features (Ellis, 1985, p.164).

Peirce (1995) claims that second language learners have "an ambivalent desire" (p.9) to speak the new language with native language speakers. With reference to this point, the children could have wished to speak with English-speaking people, but their fear of mistakes and embarrassment discouraged them from doing that. What might appear as safe strategies can thus be interpreted as the study children's lack of confidence to communicate with English-speaking people.

\subsection{Limited usage of English}

In Sarah, Luke and Eden's data, silence was not always a learning or communication strategy. I observed limited opportunities for these children to use English so that they were quiet or silent in their centres. 
Sarah's teachers interpreted her quietness in this way: "Sarah only speaks when she absolutely has to". I observed Sarah initiating conversations in English with her teachers only twice, and the following is one example of her with her teacher Casey:

Sarah finds her water bottle from the bottle bucket in the room. When she finds the bottle empty, Sarah walks to the kitchen seemingly in search for water. Sarah goes to her teacher Casey who is walking around and says: "finished", handing her bottle to Casey. Casey takes the bottle to the kitchen. (Sarah/ $3^{\text {rd }}$ visit, 11:36am)

On the second occasion, Sarah called out to a teacher "me off" after having got to the top of a monkey bar where she appeared to have trouble getting down.

In both examples, Sarah was seeking help, illustrating that Sarah might not have used the language if she had not been in a needy situation. Except for the explanations discussed in section 6.3.1.2.7, that she might have been internally learning the language and avoiding mistakes, a close analysis of Sarah's experience with the English language, also indicates that she was alone most of the time and was rarely engaged in situations when she had to talk. As will be shown in Chapter 7, although the teachers attempted to talk to Sarah, very few peers responded positively to her attempts to play with them. Sarah was observed to habitually play alone. If Sarah had few opportunities to communicate in English, it is understandable that she used it only when she had to.

Interviews with Luke and his mother provided insight into Luke's limited use of English. His mother told me: "Luke learned some English words before joining the centre and he was able to speak a bit". A reason for him not using English at the centre, according to him is that "nobody speaks to me". I observed Luke trying many strategies to communicate with English-speaking peers, but was not positively responded to. In table 6.6 is an example of him using single-word utterances with a peer but this was not actively responded to. As will be shown in Chapter 7, interacting with peers was a challenge for Luke in his centre. For this reason, it is also possible that Luke made few English utterances because his attempts to initiate interactions were unsuccessful, leading to few opportunities to use English.

Another child who demonstrated a clearly limited use of English was Eden. While Eden's mother said "I have taught Eden some English words and he might have also 
learned some from his first EC centre", and my observations of him also suggested that Eden understood some English instructions, he was rarely seen to communicate with English-speaking peers and teachers. Instead, Eden's data provided strong evidence of him speaking Chinese.

Similar to the other four children discussed in section 6.3.1.2.7, Eden's behaviours might have indicated that he was unsure of his English ability. In addition, since Eden had ready access to Chinese-speaking people at the centre and had established status in the Chinese-speaking group (Krupa-Kwiatkowski, 1998), he might have perceived that it did not matter if he did not use English. Another explanation could be peer pressure, because I noted that Eden consistently played with several Chinese boys who also used little or no English. Eden might have intended to be the same as his peers in order to maintain the connections with them. He could have been afraid that if he used English, he might lose his status in his Chinese peer group. These thoughts can be supported by Saunders (1988) who points out that peer pressure is a contributor to children's application of certain languages. While Xiaohan and Jim also had access to Chinese language in the centres, their Chinese peers all spoke English, so were less likely to exert pressure to use Chinese only. In contrast, Xiaohan and Jim might have had pressure to learn English as their Chinese peers all spoke English reasonably well.

Unlike other study children who, as discussed above, used many strategies to learn English, Eden was never seen to initiate an English conversation verbally or nonverbally, which raises the possibility that Eden was not keen on using English, or that he was not motivated to experience it. According to Ho (1998), second language learners have low motivation to learn a second language if they have little interest in what is associated with the new language, sense no relevance of the new language to themselves and have concerns about failure. If this is the case with Eden, given that he only played with Chinese peers, Eden might have perceived English to be of little use. This, accompanied by his fear of making mistakes, could have contributed to Eden's limited use of the English language.

The 'silence' of all the three children discussed in this section can be explained by some particular factors, such as the ways in which the children displayed silence and other accompanying behaviours. Like Brown (1979) and Granger (2004), I take the view that 
silence may not necessarily be an indicator of lack of learning. It could suggest children's lack of opportunities, or their low motivation to use a language. In some sense, silence can be the result of the complex relationship between language and identity in the children's learning experiences (Granger). This viewpoint is well illustrated by Eden's potential fear of losing his status in the Chinese peer group if he used English, and Sarah and Luke's choice to be silent in response to their 'invisible' status in the EC groups.

\subsection{Chapter summary}

This chapter has addressed the question of how the children in this study experienced the two languages, namely English and Chinese, in their EC centres. The answer to this question was sought through investigating the children's use of the two languages they had access to and analysing their learning experiences in relation to the use of those languages.

The data showed that all the children spoke Chinese and many strove to learn or use English. Both Chinese and English were fundamental features of the children's everyday lives and these languages connected the children's experiences to a range of learning opportunities in the EC centres. The languages, Chinese in particular, mediated the children's daily functions in communication contexts, their attempts to build togetherness with others and to seek a sense of belonging, their thinking processes and their desire for learning. The overall contribution of the Chinese language to these children's learning experiences in the centres was to help them understand the centres' programmes, learn English and develop peer relationships.

While the Chinese language was an important cultural tool for the children, their experiences with the English language had a character which, to some extent, appeared to derive from their attitudes towards the English language and towards Englishlanguage speakers, as well as their perceptions concerning the role of the two languages in their learning and development. These attitudes and perceptions could contribute to explaining why some children were able to adopt a range of strategies and were actively engaged in communication in English while in contrast, some children minimised this. The significance of these differing attitudes and perceptions lies primarily in their 
impact upon the children's learning and development as English language communicators, and also the children's learning of their positioning as Chinesespeaking children in English-learning settings.

In addition to the sociocultural factors that contributed to the study children's experiences with the languages, some experiences of the children in regards to their use of the languages were also explained by factors such as "personality, motivation, anxiety... and language proficiency" (Brown, Robson \& Rosenkjar, 2001, p.361). These factors both constrained and facilitated the ways in which the children experienced the languages.

The children's choices of the languages stood out as evidence of the range of experiences they encountered in the learning settings: inner, outer, personal, social and cognitive experiences that occurred within a pattern of interwoven socioculturallyderived practices, especially interpersonal practices. In line with the children's language choices, several findings are prominent in the present chapter. Firstly, the Chinese language was a powerful cultural tool through which all the children, regardless of their English abilities, explored a world that did not operate in Chinese ways. Secondly, most children demonstrated willingness to learn English, manifested by their active use of English and the adoption of strategies (body language, or imitation) with which to navigate an unfamiliar language context. Thirdly, the children were aware that the two languages were distinct from each other, therefore code switching was used in many communication contexts. Fourthly and very importantly, the children used languages as a practical tool to suit social contexts, which could also be tried out to meet their personal desires for creativity and language interactions.

Moreover, the children seemed aware of the value of languages as a mediator for learning and that the two languages could help them in different ways. They showed evidence of making choices about which language to use at different time, and how to use that language in order to be safe, to save face, to learn a language, to win friends and to regulate their behaviours. This suggests that language usage is an action influenced by many facets of the sociocultural context: family culture, second-language culture, home language, second language, external environment, internal motivation, and social relationships. In particular, Rogoff (1995) alerts us that "the studies of mind, 
of culture and of language [in all its diversity] are internally related" (p.140). Any language events are therefore manifestations of a cognitive structure and a cultural learning process.

In this study it is clear that both the English and Chinese languages contributed to the development of membership status for the study children in English-speaking communities and on most occasions, the children could appropriately adapt themselves to the differing cultural communication contexts. However, there was evidence of varied degrees of application of the two languages. For example, Eden demonstrated a behaviour that indicated the tension between progression in learning English and continuity in using Chinese. His 'Chinese-mostly' behaviour provided an example about how an immigrant child needs appropriate and meaningful support from people of both languages.

Through investigating Chinese immigrant children's experiences with the English and Chinese languages, this chapter identified two possible intercultural relations. Firstly, the children's use of a family cultural tool, namely the Chinese language which provided them with a bridge through which they moved from their own culture to the culture of the centres. Secondly, the Chinese and English languages could function together as common communicative devices to converge the two cultures. In the process of developing their roles in their new cultural communities, the Chinese immigrant children discovered a range of functions of their home language and at the same time, they found out how to learn about the English language. The children incorporated Chinese into the English-speaking cultural communities, so that they could rely on the 'known' language to deal with their learning of an 'unknown' language and an 'unknown' culture. If the Chinese language facilitated the children's learning experiences in their EC centres, this may be seen as an example of 'boundary object' (Leont' ev, 1981), that expanded the children's learning across the cultural boundaries within their sociocultural contexts.

In Chapter 5, I argued that New Zealand EC teachers and Chinese immigrant parents did not make significant attempts to contextualise the children's learning within their EC centres based on the children's family cultural tools. However, this chapter demonstrates that the children themselves sought and used their home language. They 
turned this cultural tool, which was not a purposefully arranged learning support by the centre or home adults, into a powerful mediator of their learning experiences in their new cultural community. Implicit in the behaviours of the children is that they were creating their own repertoire of practices through locating and applying appropriate support. It is evident that the children were active drivers of their own learning and development.

The following chapter will consider another sociocultural experience of the study children in their EC centres, namely their peer relationships. It will demonstrate how the children developed relationships with peers and how these relationships mediated their learning experiences. Given that the co-existence of the Chinese immigrant family culture with the mainstream culture of the EC centres was an important finding in Chapter 6, Chapter 7 will delve further into this experience to consider whether the 'known' culture played a role in the children's experiences with peers in an 'unknown' cultural community and if so, how that had happened. 


\section{Chapter 7: Chinese and English: Peer relationships as a tool to mediate learning}

\subsection{Introduction}

This chapter interrogates the data with a view to answering the second sub-question of the first key research question:

1.2. How do Chinese immigrant children experience their learning as a member of the group?

The study children's peer relationships, as revealed in the data, are manifested in two types of experiences: their relationships with Chinese-speaking peers, and the relationships with English-speaking peers. The children's relationships with peers of the two different cultural groups usually provided them with dissimilar mediational forces that resulted in variation in their development of group membership in New Zealand EC centres.

Theoretically, the discussion in this chapter is framed within the notion of 'peer culture' developed by Corsaro, that emphasises the importance of "peers and peer culture to children's evolving membership in their culture" (1997, p.112). In line with the central value of peer culture that sees children "doing things together" (Corsaro, 2005, p.140), the chapter analyses how (i) the study children spent time and worked together with peers; and (ii) peer relationships contributed to the children's group membership in their learning settings. Building on the notion of peer culture, this chapter will discuss social aspects of the children's learning experiences, namely their peer choices and the behaviours the children demonstrated when engaging in "activities...that [they] produce[d] and share[d] in interaction with peers" (Corsaro, 2005, p.109).

The focus on peer relationships also picks up the sociocultural idea that social relationships are a cultural mediator for learning (Daniels, 2005; Vygotsky, 1978). Specifically, this chapter draws on theoretical ideas from Vygotsky (1978) and Rogoff $(1995 ; 2003 ; 2008)$ regarding the cognitive aspect of the children's learning experiences, 
to explore how the children learned about the new cultural community in the context of their relationships with peers. Vygotsky described learning as assisted performance and Rogoff understood learning as guided participation. In particular, discussions in this chapter are anchored in Vygotsky's concept of the ZPD which highlights the importance of direct peer assistance. In addition to the analysis of the children's close interactive learning experiences with peers, discussions also utilise Rogoff's theoretical model of learning "side-by-side or [through] distal arrangements of activity" (2003, p.284). I will explore how the children experienced their learning and development in New Zealand EC centres when they were not directly interacting with peers.

Wenger (1998) makes a point of particular relevance to the focus of discussion in this chapter: learning entails changing abilities, ascribing meanings to experiences, and developing a sense of identity in the course of becoming full participants in sociocultural practices. Using Wenger's idea of 'community of practice' as an analytic tool thus raises questions about how social practices within the study centres were implicated in developing children's abilities, cultural meanings and identities.

Since the study occurred after most children had been attending the centre for enough time to have experienced interactions with peers and built peer relationships, I assume that what was recorded as the children's experiences was the result of their ongoing social contacts with peers. This thinking is consistent with the idea of Hoogsteder et al. (1998) that "each interaction is a construction, to which the participants bring...the previous history of their relationship" (p.179).

As mentioned in the beginning of this chapter, the children's relationships with their Chinese-speaking peers are an important focus of this chapter. It was already evident in Chapter 6 that many children played with Chinese peers. In that chapter, peer experiences were touched upon within an analysis addressing the question of how the children experienced the two languages in their environment. In this chapter, I will strengthen this exploration. Within the context of peer culture, and from a sociocultural perspective, I will investigate how the children and their Chinese peers collectively produced and participated in peer practices that manifested the influences of their family culture. 
This chapter will also consider peer relationships between the Chinese immigrant children and their English-speaking peers, who were an integral part of children's experiences in their centres. Chapter 6 has shown the influence of English-speaking peers on the study children's English-learning experiences. In this chapter, I look at how the children developed their group membership in the centres through relating to English-speaking peers, or in hope of relating to them. This analysis is conducted in a way that illuminates how the Chinese immigrant children's peer-learning experiences in the EC centres drew on both the culture of the EC centres and that of their families.

\subsection{Relating to Chinese peers}

Relationships with peers from one's own cultural group are central to immigrant children's development of membership in mainstream cultural groups (Denscombe, Szulc, Patrick \& Wood, 1986; Feng et al., 2004). In my study, relationships with Chinese peers emerged as the basic fabric of the daily experiences of the study children who had Chinese peers in their centres. There were numerous examples across the data of the study children paying attention to Chinese peers, interacting with Chinese peers, and playing alongside them. The analysis of these examples indicates three types of experiences commonly encountered by the study children through their interactions with Chinese peers:

(i) speaking Chinese and being Chinese as the main criterion for friendship;

(ii) obtaining a sense of belonging and togetherness as a small group within the larger English-speaking cultural communities; and

(iii) developing and using social skills.

\subsubsection{Speaking Chinese and being Chinese as the main criterion for friendship}

As mentioned in Chapter 6, six of the eight participating children had Chinese peers in their centres. All of them were observed to have lengthy and engaged interactions with a Chinese peer or peers. Eden displayed an exclusive pattern of playing only with Chinese peers. When asked who they wanted to play with, replies were: 
Xiaohan: Chinese children, we can all speak Chinese. We are friends.

Leah: I have many friends. I have Chinese friends. I have other friends too.

Eden: $\quad$ I like playing with Jo [a Chinese child]

Rick: $\quad$ I like Xiaohan and Peter [Chinese children]. They are my friends.

Jim: $\quad$ I play with Leah [a Chinese child].

Amy: $\quad$ Ken [a Chinese child] wants to play with me. I play with Ken.

In addition, in reply to "what do you think friends do", two children made the following points:

Xiaohan: $\quad$ speaking Chinese

Rick: $\quad$ all being Chinese

It is evident that the Chinese immigrant family culture played a role in orienting the children's peer preferences and language choices because the children perceived Chinese language and cultural background to be the key ingredients of desirable peer relationships. These views are reinforced by one of the findings identified in Chapter 6 , that the use of Chinese with Chinese-speaking peers was dominant in the centre experiences of many Chinese immigrant children. The children here articulated the reasons for them to develop first-language and first-culture oriented peer relationships, thereby indicating that being Chinese and being able to speak Chinese were important criteria for their friends. When explicitly linking the word 'Chinese' with the word 'friends', Leah (4:6/AC), Rick (4:5/AC) and Xiaohan (4:8/M) expressed a belief in the importance of Chinese language and cultural background for their choice for friends. These three children were older than the other study children and this may have enabled them to be more articulate in using Chinese to indicate the importance of their family culture for their friendship choices.

The children's reasoning for their choice of peer relationships as the same language and cultural background demonstrates that similarity is an essential element in their social choices. MacDonald (1996) claimed that "children are attracted to peers who are similar to themselves on a wide variety of traits" (p.53). As in the study by Feng et al. (2004), the children in my study displayed a strong desire and willingness to be with peers of 
the same cultural backgrounds in their new cultural context of a New Zealand EC centre. This evidence reveals that the culture of Chinese immigrant families is helpful in forming the children's peer relationships in non-Chinese cultural communities.

\subsubsection{Obtaining a sense of belonging and togetherness as a small group within the larger English-speaking cultural communities}

According to Kemple (2004), children receive a message that "We all belong here, together" (p. 51), when they are accepted by others. I discussed in Chapter 6 the study children's attempts to seek a sense of togetherness with Chinese peers through their use of the Chinese language. This phenomenon is even more conspicuous when the data are viewed in terms of the children's specific behaviours as they interacted with Chinese peers. There were many scenarios in which the children demonstrated that being together with Chinese peers raised their awareness that they had a place in the centres, thus giving them a sense of belonging.

The extracts in Table 7.1 below capture a few pictures of the children seeking out togetherness with their peers. 


\section{Contexts}

Xiaohan walks around in the centre, looking around. (Xiaohan/2 $2^{\text {nd }}$ visit, 9:16am)

Jim sits at the bed in the family corner, watching the children playing in the room. $\left(\mathrm{Jim} / 1^{\text {st }}\right.$ visit, 11:08am)

Eden is playing in the 'playhouse' outside. Joe (Chinese peer) is walking in with his dad.

(Eden/ $3^{\text {rd }}$ visit, 8:42am)

Rick is crawling on the floor. (Rick/3 $3^{\text {rd }}$ visit, 1:49pm)

\section{The children's behaviours}

Xiaohan: "Rick, Ben, zai na ne?" [Rick, Ben where are you?]

Jim murmurs with tears in his eyes: "lean, leah, ni zai na?" [Leah, where are you?]

Eden calls up as soon as seeing Joe: "Joe, wo zai zhe" [Joe, I'm here].

Rick jumps up at the sight of Ben and runs to him: "Ben, ni shui xing la. Dao wai bian hao ma?" [Ben, you are up. Will we go outside?]

\section{Peer responses}

Ben (seeing Xiaohan): "zhe li, dao zhe lai ba" [here, come over!]

Ellie (Chinese teacher), possibly hearing Jim, finds Leah: "Leah, Jim is looking for you".

Leah goes to Jim.

Joe: "ma shang jiu lai" [coming shortly].

Joe takes his bag to the room and runs to Eden.

Ben: "hao ba" [okay].

These examples illustrate that the study children looked for their Chinese peers (Xiaohan and Jim's examples) and appeared excited when they saw them (Eden and Rick's examples). Corsaro (2005) identified "doing things together" to be a central value of peer cultures (p.140). Like the study children in Corsaro's study, the children in this study sought out their peers because these relationships provided them with a sense of presence and a sense of belonging in their learning settings (Long, 1997).

Underlying the sense of togetherness could be a sense of reliance and mutual acceptance that was evident in the observed interactions among the Chinese peers in the study. As shown in Jim's example, he looked for Leah when he was crying, probably because he knew that Leah could be relied upon to comfort him. Xiaohan's query, Eden's invitation and Rick's suggestion all appeared to be made with confidence that their peer would accept them with the same enthusiasm as they welcomed their peers. 
As mentioned also in section 7.1.1, three children, Leah, Xiaohan and Rick, who are fours years of age, regarded their Chinese peers as friends. Young children understand friends to be close companions who play with them (Howes, 1996). So, naming specific Chinese peers as friends with whom they had initiated frequent interactions indicates the children's need for close peer relationships and the ability of their Chinese peers to help them meet these needs.

Because togetherness is "a complex tendency of forming and maintaining a group" (van Oers \& Hännikäinen, 2001, p.102) underpinned by a perception of "we-ness" from the group members (Hännikäinen \& van Oers, 1999, p.6), when being together, children need to mark their own identities within the groups and define who should be in the groups. This point has been raised in Chapter 6 (see section 6.2.1). Using Xiaohan and Leah's examples, in which they included the concepts of 'us' and 'our' in conversations with Chinese speaking peers and adults, I discussed 'we-ness' as an indicator to divide group members from the non-group members. In the following excerpt from my field notes, the children's sense of togetherness with peers and their group identities emerge even more clearly.

Rick climbs onto a plank where Peter (Chinese) is walking. Rick starts walking behind Peter. Peter leaves the plank and runs to the playhouse on the other side and calls: "Rick, lai ya" [Rick, come here], poking his head out of the playhouse. Rick gets off the plank and goes to the playhouse. On the way, he sings a song in Chinese: "du....". Rick enters the playhouse and sits on the floor. Peter sits beside him. Xiaohan finds them and pushes herself in. Xiaohan climbs out of the window and so do the other two children. Rick: "wo men dao na hao ma?" [Shall we go there?], pointing to the other side of the yard. He runs and so do Xiaohan and Peter. Rick then runs back to the playhouse. Xiaohan and Peter follow him. The three of them go and sit in the playhouse. Ben (Chinese) goes to them too. All these children sit next to each other on the floor. Rick stands up, putting his arms around them all. He holds Ben's hand, Ben holds Xiaohan's and Xiaohan holds Peter's hand. The children move out of the playhouse and walk to the bridge that links the playhouse to the ground. They sit down on the bridge. Tony (English) comes over and stands on the ground watching them. Ben bends down and says to Tony: "you cannot come to OUR house". He turns to the Chinese peers: "dui ba, zhe shi wo men de jia" [right? This is our home]. Xiaohan: "dui" [yes, you are right]. Ben then turns to Tony: "go". Tony shouts: "no". Ben kicks him. Xiaohan: "shi wo men da jia. Rang ta zou" [this is our home. Let him go]. She then stands up and waves her fist at Tony. Rick: "zou ba, dao fang li qu" [let's go to the playhouse]. Ben still kicks Tony. Xiaohan shouts: "go, go". Peter puts his fist up towards Tony. Tony cries and leaves them. Rick walks to the playhouse and the peers follow them. (Rick $/ 4^{\text {th }}$ visit, 3:20pm) 
This excerpt is interesting for two reasons: the children followed one another closely and demonstrated a sense of belonging to their group by using the words 'us', 'our', 'we', as well as moving together and Rick cuddling them all; Ben, Xiaohan and Peter were fighting as a group against Tony. Ben and Xiaohan referred to the 'bridge' and possibly 'the playhouse' as 'our home', thereby demonstrating a clear sense of we-ness, connection and unity. As discussed in Chapter 6, togetherness was in some way built upon a common language. The example here indicates that togetherness could also be developed by an attempt to belong to each other and to a shared place (Hännikäinen, 1998). Another way to see their sense of group at work is to consider the children's fight with Tony. The children did not include Tony in their group possibly because Tony could not speak their language, was not a regular play peer for any of them, and the children were in 'their own' shared place. Moreover, if the children had likened the playhouse to their 'home', Tony, a non-Chinese, would have been regarded as an outsider, who, therefore, should not have come in without being invited. What the Chinese immigrant children did here is a typical example of the maintenance of a 'core group' in peer cultures, in which the group members "often work together to resist the entry of new members... simply because they are not members of the group" (Corsaro, 2005, p.185). Rick did not join the fighting but suggested a change in the context. An explanation for his behaving as an onlooker could be that as his mother and the teacher said: "Rick is very gentle and calm", so Rick did not like fighting with others. Even so, his words 'Let us...' also indicated Rick's attempt to move the group together. What the children did in this scenario illustrates that for them, becoming a member of a group means finding ways of being together as well as stopping other people (non-group members) from entering their group.

Although the children moved around numerous play areas, the places they used frequently with their close peers were the playhouse (Rick, Xiaohan and their friends), the family corner (Rick, Xiaohan and their friends), a table and chairs on the corner of the playground (Leah and her friends), animal play corner (Amy and her peer) and a playhouse (Eden and his peers). I noted Xiaohan searching for her peers in the playhouse on a few occasions and Eden walking up to the one in his centre and asking, 'where is Joe?'. It is important to note that all these areas were on a corner of the playground or the play room. A common feature of these areas was that they were not prominent and were somewhat distant from the popular areas of the centres. Therefore, 
it is possible to infer that these children chose specific places to be with each other where they were free to do whatever they wanted without being noticed too easily. Feng et al. (2004) also found that when the Chinese immigrant children in their study were away from the busy areas of the centre, they practised what they were familiar with and their interactions were of an engaged nature. Corsaro (2005, p.141) called these places “interactive spaces" and their regular and frequent use could be the result of children's intention to be in control of their interactions. When coming together, children aim to maintain group status and to be in control of their actions in their own interactive space'. To achieve this, the study children initiated and maintained peer interactions through their own approach and also adopted various means of maintaining their peer groups. The above example of Rick and his peers refusing Tony's entry, as a non-group member into their group, illustrates this process.

When considering all the scenarios that illustrate Chinese children being together in their interactive space using only their first language, there is also a sense of the children attempting to keep their family culture apart from the mainstream culture of the centre. So, despite the prominence of 'Kiwi' culture, the study children and their Chinese peers created an independent status for their family culture through their choices of a particular space, particular language and particular peers. The figure below illustrates yet another intercultural relationship between the Chinese immigrant family culture and the mainstream culture of the EC centres. In this relationship, the family culture was kept apart from the EC culture because as Rick said, "we are all Chinese children" (see section 6.2.1). This indicates that alongside the children's desire to settle and belong was also a desire to maintain their own cultural identity. 


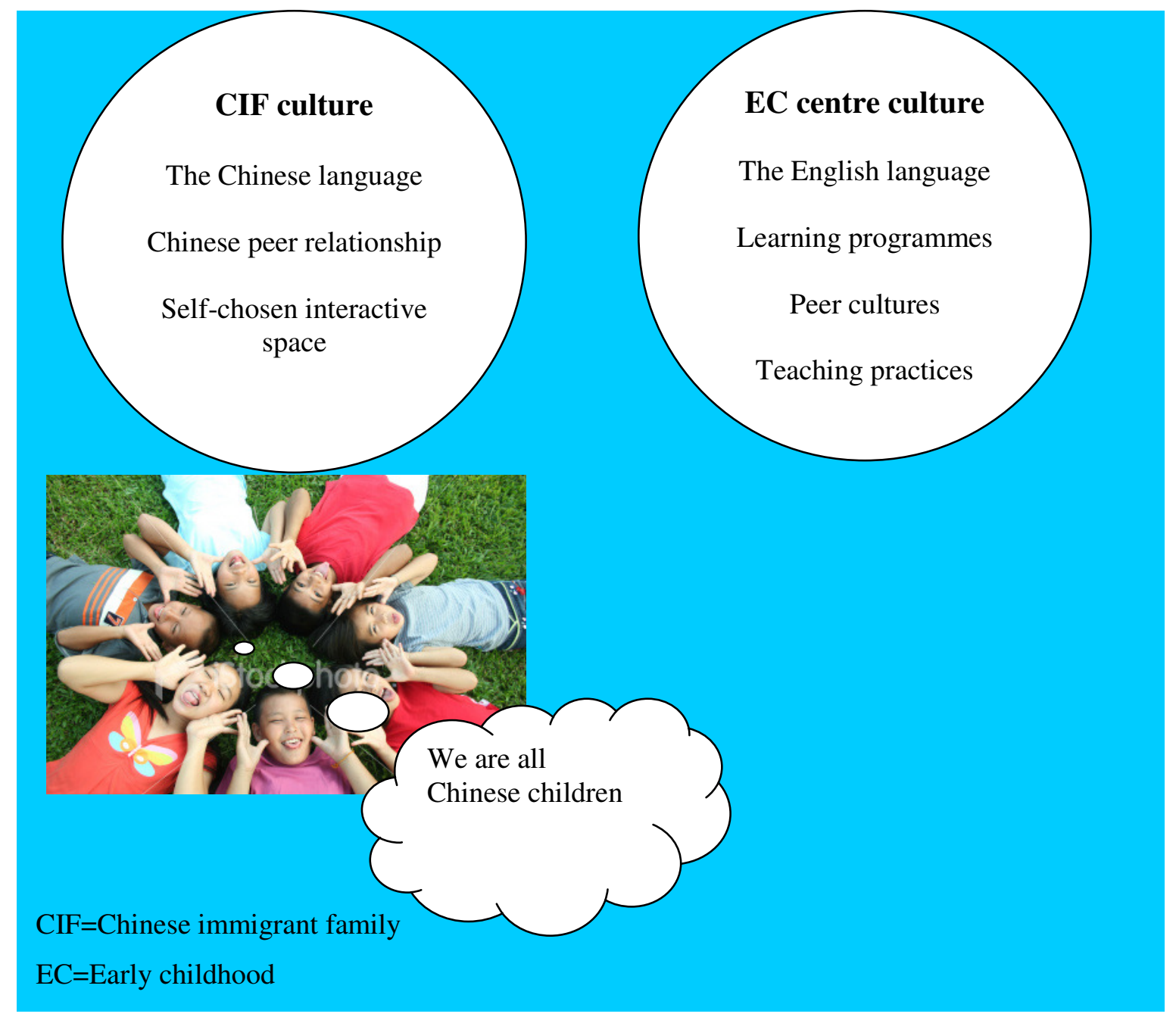

Figure 7.1: CIF culture being kept apart from the EC centre culture

What also became evident in the 'together' examples of the children is that they, especially those who were newcomers, gained knowledge from their Chinese peers about the rules, routines and rituals of their new cultural communities. This is important, given the aspiration of Te Whäriki that "children... feel a sense of belonging, [when] "they feel comfortable with the routines, customs, and regular events [and] they know the limits and boundaries of acceptable behaviour (Ministry of Education, 1996, p. 54). For this reason, it is possible to argue that regular participation in Chinese peer groups contributed to the children's sense of belonging to the larger learning contexts of the EC centres.

Analysis of the observations identified imitating peers to be a useful contributor to the children's development of understanding about the centres. Some children, for example 
Eden $(3: 3 / \mathrm{M})$, put a hat on before going outside after he saw his close peer Joe do this. In Eden's centre, "putting the hat on is a rule for children playing outside" (Eden's teacher Sarah), and he learned this rule from Joe. After that occasion, I observed Eden putting his hat on every time he went outside. Xiaohan (4:8/M) also closely followed Rick (4:5/AC) on some group-learning occasions, and I observed her imitating Rick's every move during a 'mat time' activity, when the children were gathered together to play with a teacher.

Verbal instruction or guidance of Chinese peers was the most identifiable support for the study children's learning about the centres. On one occasion, I observed Joe, Eden's Chinese peer, explaining to him what their teacher Rebecca had said to them about an excursion.

On his first visit, I noted several occasions of Jim (3:1/M) moving with Leah (4:6/C), his older Chinese peer during teacher instructions. The excerpt below is an example:

Helen (teacher): "come on, mat time".

Jim is playing blocks. Leah is drinking.

Leah puts down her bottle and goes to the older children's room, sitting on the mat. Jim follows behind her and sits next to Leah.

Leah sees Jim: "Jim, ni dao na ge fang jian" [Jim, you go to that room], pointing to another room.

Jim raises his eyebrows.

Leah: "zhe shi da hai zi de wu. Ni men xiao de qu na ge wu" [this is big children's room. You little ones go to the other room].

Leah gives Jim a push. Jim stands up, leaving the room and goes to the younger children's room. $\left(\mathrm{Jim} / 1^{\text {st }}\right.$ visit, 11:51am)

Initially Jim did not appear to have understood Leah's intention, but moved in response to Leah anyway. Later I saw him asking Leah: "wei shen me wo yao dao na ge wu qu ya" [Why should I go to that room?], and she explained that when a teacher asked them to move, Jim should go with the teachers Joy or Helen because they were his room teachers. Leah showed Jim who Helen and Joy were. After that, Jim always went to the correct room during 'mat time'. Clearly, Leah had helped Jim understand what to do during a routine activity.

Similar to Leah telling Jim not to sit in her room during mat time, Xiaohan's peers also helped her understand what she should not do: 
Xiaohan is outside playing with Ben and Peter. A baby on the other side of the building is crying. Xiaohan walks over and puts her leg over the fence, in an attempt to go over the fence to see what is happening. Ben runs to her and pulls her back: "bu neng sui bian dao na bian qu de" [cannot go that side]. Xiaohan stops and comes back. (Xiaohan $/ 5^{\text {th }}$ visit, $\left.3: 11 \mathrm{pm}\right)$

In Xiaohan's centre, the baby room is adjacent to the preschool. The children, however, were not allowed to visit each other freely unless the teachers accompanied them or granted permission. As Ben had been at the centre for two years and he knew the rules, in response to Xiaohan's attempt to move over the fence, he told Xiaohan it was not allowed. Xiaohan complied.

The data indicate that when learning about the centres from their peers, the study children used their first language. Implicit in this is the idea that the children's first language shared with their Chinese peers was crucial to their learning about how the centres operated. In Chapter 6, I also discussed how the children used their first language to seek help from the Chinese peers (see section 6.3.1.2.4).

Underpinning this acceptance of the rules is also the children's trust in their peers. They knew their peers were helping them. This indicates that peer relationships facilitated the children's transition to their centres, contributed to their willing acceptance of a new life, and helped them develop a sense of belonging. For these reasons, it is necessary to note again that peer relationships in small groups, mediated through the children's joint use of the Chinese language, were central elements in their development of a sense of group membership in the EC centres.

Analysis of the study children's use of English provided further evidence regarding Chinese peers supporting their learning about the centres. In Chapter 6, I explored the children's imitation of their Chinese peers' use of English and their requests for help from Chinese peers to understand English instructions. Developing their English language was another important aspect of the children's peer interactions related to their learning about the centres, because English was the predominant language there and its development was critical for the children's establishment of a sense of belonging.

Peer guidance is an essential element in sociocultural theories, in which Rogoff (2003) and Vygotsky (1978) stressed the importance of cultural partners for enhancing learning 
and development. The examples of learning from peer interactions above strikingly resemble these theoretical ideas. The children's imitation of their Chinese peers, their use of Chinese to seek support, and learning English together are specific ways in which the Chinese peers assisted the study children's learning and development in another cultural community. It is clear that the Chinese children as a group produced their own means of existence in non-Chinese cultural communities by retaining and bringing to life the cultural modes of representation from their families (Wartofsky, 1979). Chinese immigrant family culture represented by peer preferences assisted the children to master their functions in non-Chinese cultural communities.

Another great strength of the peer-learning groups that the Chinese immigrant children formed lies in the quality of the peer interactions and activities that were tailored to the children's needs, not only for their establishment of group memberships, but also the development of their own meanings of the learning experiences (Wenger, 1998). The regular learning opportunities with their Chinese-speaking peers drew them together in a community of practice. In this way, the children might have ascribed the meanings of their experiences in the EC centres to be, for example, doing what their Chinesespeaking peers were doing, speaking Chinese, and regularly playing with their Chinesespeaking peers.

If Chinese peers mediated the children's learning, then it is significant to note that the children 'produced' or 'created' the learning opportunities for the mediation to occur, and these opportunities could be as explicit as verbal communications, or tacit as quiet imitations. While it is easy to observe how the children used verbal communications to learn with peers, and their verbal requests for help are a good example of this, quiet imitation of peers as a self-created learning strategy warrants special attention. I discussed in Chapter 6 how Vygotsky (1987) understood imitation as a learning activity, which entails an active internalization by less capable learners of what their more expert partners provide. Rogoff (2008) agrees and expands it to view imitation as a social action too. A significant argument she made about children's learning and development is that "their actions are deliberated...often in an opportunistic, improvisational fashion" (p.64). Rogoff contends that learners motivationally and strategically take responsibility for positioning themselves to participate. This is illustrated in the current research in the ways the children sought as well as created social opportunities for them to learn from 
their peers. Peer companionships or interactions, in this sense, are learning sources that the study children made conscious attempts to use, to find a place, to develop confidence with the centre programmes, and to understand acceptable behaviours in the new environment (Ministry of Education, 1996). The children in this study are thus active agents, capable of relating different cultural tools to create their own ways of learning and development.

To develop and maintain relationships with peers, children need to develop and adopt appropriate behaviours, in order to benefit others (Knafo \& Plomin, 2006). Hartup (1996) says: "Children behave cooperatively in order to become friends, but also to remain friends" (p.223). Consistent with Hartup's view, analysis of the children's data revealed their use of such social skills as negotiating and making compromises, self assertion, helping and taking care of peers.

\subsubsection{Learning to negotiate and compromise}

Because children bring a wealth of individual differences with them, learning to reconcile their own needs with those of peers is essential in maintaining relationships. This, as Parker and Gottman's explain (1989, p.112), is that "if play is to be coordinated, it is simply not always possible to get one's own way. In service of the overall adventure, children must inhibit some actions [and] accept influence at times".

The application of negotiation skills and making compromises was a clear theme regarding the study children's interactions with their peers. In many cases, the children negotiated taking turns, as in the following excerpt when Rick both co-ordinated turn taking and used negotiation to reach a compromise.

Ben (Chinese) is pushing a bike that has a seat at the back.

Rick walks around. Rick walks to Ben. He holds the bike to make it stop and asks: "ni tui wo ma?" [Will you push me?].

Ben shakes head. Rick: "na wo tui ni ba" [then I push you].

Ben lets the bike go and walks to sit at the back of the bike. Rick pushes him.

Rick (2minutes later): "ni tui wo ma?" [Can you push me now?]

Ben gets off the bike and Rick gets on. Ben pushes him. (Rick/2 ${ }^{\text {nd }}$ visit, 11:25am)

A further instance of the children's turn taking and compromise was provided in Xiaohan's interview data. When asked how she plays with peers, she said: "sometimes I need to listen to them". Although Xiaohan's mother described her as a very self- 
centred child, this reply demonstrated Xiaohan's awareness of the importance to adjust her own interests and needs to those of her peers. In my own observations of Xiaohan, I noted many examples of her negotiating and reaching compromises to maintain her status in the peer group. One is presented as follows:

Xiaohan is building a structure with blocks. Rick walks up to her to watch.

Xiaohan: "gei wo yi gel an se de" [give me a blue one], pointing to the block pile.

Rick finds a blue one and gives it to Xiaohan.

Xiaohan: "wo yao yi ge da de" [I need a big one].

Rick: "zhao bu dao" [I cannot find it], "ni zi ji zhao ba" [get it yourself] and leaves.

Xiaohan: "Rick, hui lai. Wo jiu yong ni de lai se de" [Rick, come back. I use the blue one you gave me].

Rick walks back.

Xiaohan smiles: "Rick, ni bang wo zhao yi ge huang de hao ma?" [Rick, will you find me a yellow one?]

Rick: "da de?" [a big one?]

Xiaohan gives a smile: "sui bian" [up to you]. (Xiaohan $/ 5^{\text {th }}$ visit, 3:26pm)

In this example, Rick appears to have taught Xiaohan that she would lose her friend if she was not nice to him. To keep Rick as her friend, Xiaohan appeared to have changed the communication style from giving orders to negotiating and making compromises. In her centre, Xiaohan demonstrated quite a strong reliance on some peers. Despite her mother's statement that she was self-centred, Xiaohan was clearly able to adjust to peers in order to live and settle in the environment.

In view of the above examples, it seems that key to the children's negotiation and communication was not only their ability to speak but also their sensitivity and attention to the needs of their peers. Accompanying this could also be their attempts to find a common ground or interest. The children were trying to be "in tune" (Kantor, Elgas \& Fernie, 1998, p.133) with their peers but at the same time wanted to put their own wishes into practice. Lofdahl (2006) concludes that "children use both bodily and verbal communication to negotiate roles and actions" (p.79). The examples in this study support this and extend it by illustrating that through experiences with their peers, the study children became aware that they should communicate appropriately to maintain their relationships.

What is of interest in these children's active negotiations with their Chinese peers is that they were unlikely to follow a traditional Chinese way of communicating, which has been documented as being restrained, inhibited and characterised by quiet behaviours 
(Chen, Rubin \& Li, 1997; Chen, Rubin \& Sun, 1992). By contrast, the data in my study demonstrates that the children were active and autonomous communicators with their Chinese peers. What could this phenomenon imply? The question may be answerable in two ways. Firstly, in their new environment, the Chinese immigrant children were observing active communication by many other children and they embraced this cultural practice. The children were responding to a context in which they were expected and encouraged to communicate and show agency. Secondly, Chinese cultural tradition regarding social behaviours may be changing in the Chinese immigrant families. Thus, a key implication of my research is that for the study children, social learning experiences were mediated not only by their family culture but also by the culture of New Zealand EC centres. Moreover, these findings also indicate that traditional cultural orientations that predicted Chinese children's behaviours cannot be fully relied upon. Because culture is continually evolving, it is important to be alert to existing sociocultural environments when seeking to understand children's learning experiences (Cole, 1998). It is therefore necessary to once again emphasise the importance of referring to 'Chinese immigrant family culture', but not the generic term of 'Chinese culture' to talk about the children's learning experiences in this study.

\subsubsection{Using assertive communication to accomplish their own wishes}

Another noteworthy skill that the children developed and used with their Chinesespeaking peers was assertive communication through which they accomplished their own wishes. MacDonald (1996) argued that close relationships with peers do not just involve self sacrificing but also satisfying one's interests, meaning that inherent in peer relationships is a focus on self too. The following extracts are drawn from the present study to demonstrate the children's use of assertive communication to accomplish their own wishes. 
Table 7.2: Children's assertive communication to accomplish their own wishes

\section{Contexts and the peers Data of 'accomplish own wish' \\ Amy is drawing at a table. Ken goes to her, takes hold of Amy's hand and pulls her towards the door. (Amy $/ 3^{\text {rd }}$ visit, 2:49pm) \\ Amy: "Ken, ni yao gan shen ma" [Ken, what do you want to do?] \\ Ken: "na li wan" [go there], pointing outside. \\ Amy: "bu, wo yao hua hua" [no, I want to do drawing]. \\ Ken pulls Amy out. \\ Amy: "bu. Wo bu qu" [no, I am not]. \\ Ken gives up and leaves Amy.}

Xiaohan is painting on a sheet of paper. Ben goes over to watch. Ben uses the paint to draw a circle on Xiaohan's work. Xiaohan stops painting. (Xiaohan $/ 4^{\text {th }}$ visit, 10:56am)

Xiaohan: "Ben, ni zai gan shen ma?" [Ben, what did you do?]

Ben: "bang ni ya" [helped you].

Xiaohan: "hao chou, wo bu xi huan" [too ugly, I don't like it].

Ben shrugs his shoulder.

Xiaohan: "ca xia qu" [clear it].
Ben: "zen ma ca?" [how to?]

Xiaohan: "ca diao" [wipe it].

Ben: "wo bu hui" [I don't know how].

Xiaohan: "bu, ni yao zuo, wo de hua bu piao liang le" [no, you must do it. My picture is not beautiful anymore]. Ben: "wo qu ca bu" [I fetch the cloth]. Ben comes and wipes the mark.

Rick is making dough at the dough table with Ben. (Rick/2 ${ }^{\text {nd }}$ visit, 9:21am)
Leah is running outside.

Fang is dashing to Leah and suddenly cuddles Leah from behind and pushes Leah down to the ground. Leah's knee is bleeding. Fang stands and looks unsure of what to do. $\left(\mathrm{Leah} / 5^{\text {th }}\right.$ visit, 2:13pm)

\section{Explanations}

Amy insisted to Ken that she wanted to stay inside because she wanted to do her drawing.

Xiaohan sustained an attitude that Ben needed to clear the mark because he did it without asking for Xiaohan's permission and Xiaohan did not like it.

Rick was insistent that Ben needed to put the 'head' back on Rick's gun because Ben had broken it.

Leah asserted that she had a right to get an apology from Fang because Fang hurt her even if Fang was a very close friend of hers. 


\subsubsection{Helping and taking care of peers}

Another social skill that the study children used in their peer relationships was to help and care for each other.

On a few occasions, Leah assisted Jim to find his way around and responded to Jim's needs, for example, reading a book with Jim that he handed to her. Amy (3:6)'s "best friend Ken" (Amy's interview) was a Chinese boy aged 2 years 2 months. In many instances, Amy positively responded to Ken's need to be with her. She said during our interview: "Ken wants to play with me". I noted an incident that took place when a photographer who visited Amy's centre left his case on the floor:

Amy and some other children are banging the photographer's case on the floor. They are laughing.

Ken stands in the middle of the room, calling up: "Amy". Amy quickly stops banging, turning around, standing up, going to Ken and taking Ken's hand. (Amy $/ 3^{\text {rd }}$ visit, 9:29am)

Amy's quick response to Ken indicates that she was mindful of Ken and that helping Ken to meet his needs was important to her. Amy was also observed to save a chair for Ken next to her own during lunch time and when an English-speaking peer attempted to take it, Amy simply said to her: "Ken".

It is also interesting that although $\operatorname{Jim}(3: 1)$ was younger, he also supported a Chinese peer, Wayne, who was 4 years and 6 months at that time but quiet and withdrawn. I recorded the following:

Wayne is sucking his fingers behind the door of the block room. Jim comes out of the block room. He sees Wayne. Jim walks out to Wayne, reaching for Wayne's hand and leads him to the block room. $\left(\mathrm{Jim} / 4^{\text {th }}\right.$ visit, 11:45am)

Later when the two boys went outside, Jim took Wayne to the playhouse and after sitting down on the bench there, Jim said to Wayne: "zuo dao zhe ba" [let's sit here], pointing to the space beside him for Wayne to sit. Jim and Wayne played together for 46 minutes on that occasion with Jim playing the role of a carer. After that, Jim was approached by Wayne and they played together a few more times. Obviously, Jim supported Wayne's learning experience in their EC centre, which led to Wayne becoming close to Jim and enabled the relationship between them to develop. Jim's 
support of Wayne corresponded precisely to Leah's behaviour towards Jim. This was apparent to me because when I observed Jim helping Wayne on my fourth visit, it was two months after Jim had started attending the centre and he had experienced much 'help' from Leah. It is very likely that the relationships that Jim developed with Leah contributed to his understanding of the reciprocal features of a peer friendship. From his experience with Leah, Jim might have understood that he became close to Leah, because Leah had helped him and if he did this for Wayne, Wayne would become close to him too. In some sense, this example is representative of a friendship construction. Jim developed a new peer relationship based on his experiences in another peer relationship (Corsaro, 2005).

What also emerged in the data of some children, such as Rick, Xiaohan and Amy, was that they were mindful of their Chinese peers and cared for them even when they were not close to them. The relationships between the children in this study and their Chinese peers are good examples of the proximal and distal ways of learning and development that Rogoff (2003) suggested operate in children's cultural communities. The following excerpt illustrates Rick's and Xiaohan's mindfulness for their Chinese peer, Ben.

Rick is playing at the dough table on his own. Xiaohan is standing by the kitchen door, watching May preparing drinks. Suddenly a scream is heard.

Rick (murmurs): "Ben". He quickly leaves the dough table and goes in the direction of the screams.

Xiaohan follows behind Rick.

Ben has hurt himself when running outside. Jenny (teacher) makes him sit down and puts a plaster on his knee.

Rick goes to Ben: "mei shi ba?" [Are you okay?]

Ben nods.

Xiaohan: "teng ma?" [hurt?]. (Rick/2 ${ }^{\text {nd }}$ visit, 2:09pm)

Xiaohan and Rick rushed to check up on Ben after his injury. This indicates both children's attention to their friend's wellbeing and their care for a friend. This incident is similar to the example I noted earlier in this section that illustrated Amy's quick response to Ken when Ken called her from a distance. I also observed Amy's quick response to Ken when he cried after a teacher stopped him from throwing toys. On this occasion, Amy was not physically close to Ken so had not observed what caused the crying but she ran to him promptly when she heard Ken crying, coaxed him to sit down, and sat with him. 
These behaviours of the children are consistent with the findings of other researchers who have argued that children's friendships are voluntary in nature (Deegan, 1991; Ladd \& Kochenderfer, 1996; Lofdahl, 2006). They were together in many situations but were able to make their own choices. What is evident is that these children sought out their peers and they perceived the peers to be important. They established peer relationships that included features of both physical and emotional proximity (Howes, 1996), and empathy, faith and special attention to each other (Hännikäinen \& van Oers, 1999). It is difficult to decide whether these skills were acquired from the children's interactions with peers, or through observing ongoing experiences at home or in the centres (Rogoff, 1995). However, it is clear that by demonstrating these behaviours, the study children adapted gradually to the social world by paying attention not only to their 'self' but also considering the feelings and needs of their peers.

As shown in this section and section 7.1.2, the relationships that the study children had with their Chinese peers mediated the children's learning in a range of ways, including the development of knowledge about the centre programmes, of the English language and social skills. The figure below illustrates how the two sets of cultural systems became related through the study children's experiences with their Chinese peers. This is another example that illustrates the Chinese cultural tools acting as a bridge for the children's involvement in their EC cultural communities.

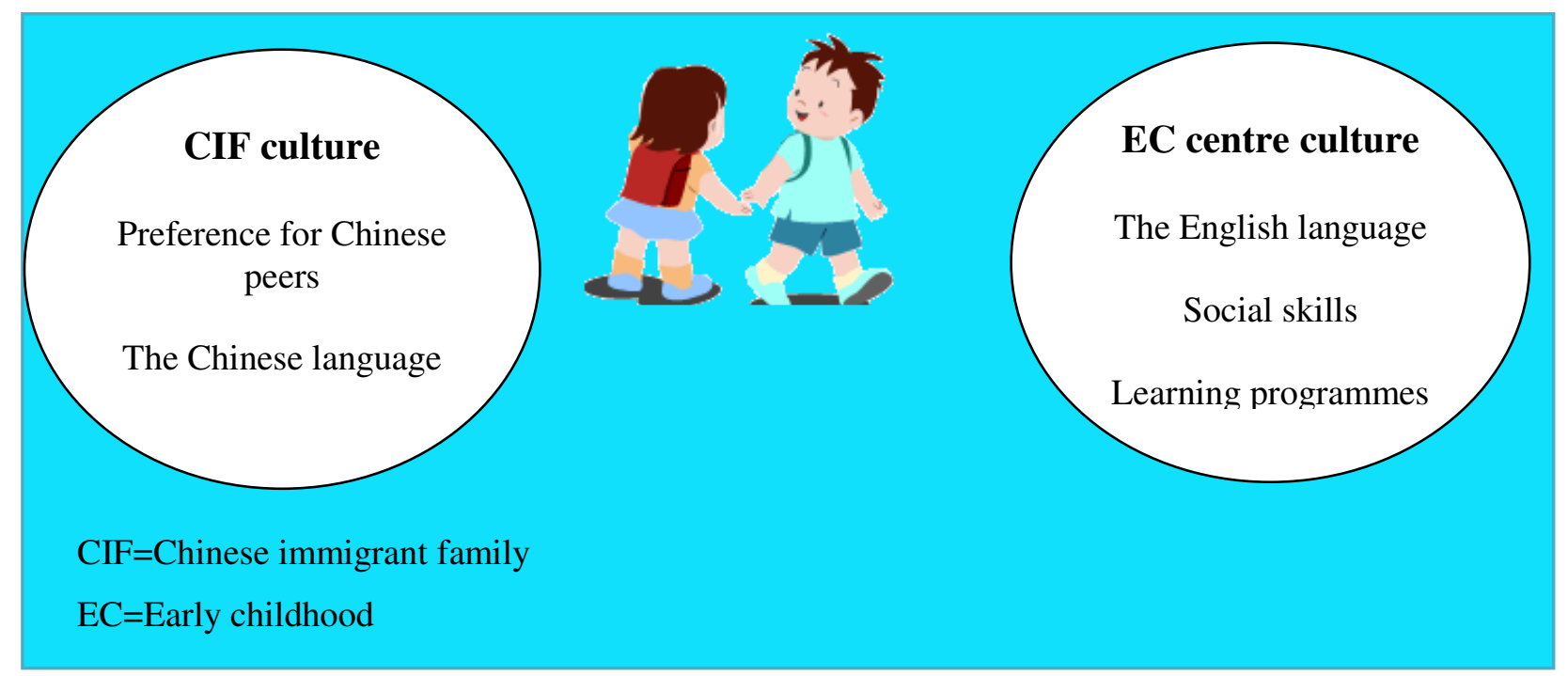

Figure 7.2: Chinese-Chinese peer relationships to bridge the two cultures 
An affirmation of the language and cultural identities of the Chinese immigrant children in their Chinese-speaking peer groups also has to do with their experiences of crossing the boundary zones (Wenger, 1998). The particular value of the Chinese-speaking peers became obvious when the children used them as 'boundary objects' to coordinate their learning across cultural settings. The function of the Chinese-speaking peers was similar to that of the Chinese language, acting as a means of helping the children transfer and expand their learning and development.

\subsection{Relating to English-speaking peers}

The previous section discussed the importance of Chinese peers in mediating the study children's learning experiences in English-speaking EC centres. The analysis of the children's relationships with their Chinese peers illustrates how these children socialised and learned with each other in proximal and distal ways. However, there remains the issue of how the children established relationships with English-speaking peers, who were an essential part of their peer groups, and how these peer relationships contributed to the children's learning experiences in the EC centres. Also, English-speaking children were the only peers that Sarah and Luke could interact with in their learning settings, since their centres did not have any other Chinese-speaking children attending. The present section focuses on this aspect of Chinese immigrant children's learning experiences.

Three key themes emerged from the analysis of the children's relationships with English-speaking peers:

(i) learning with English-speaking peers was a desirable but complex experience;

(ii) relating to English-speaking peers with caution; and

(iii) aggressive reactions to peer conflicts.

\subsubsection{Learning with English-speaking peers was a desirable but complex experience}

According to Corsaro, the key to peer relationships is the enjoyment that children can gain from being with each other because "children immensely enjoy simply doing things together" (2005, p.158). In an earlier study, Lieberman (1977) more explicitly 
said that "young children have been found to display interest in peers" (p.1277). In my study, there is evidence that the children were interested in playing not only with Chinese but also their English-speaking peers. I noted scenarios in which Leah (4:6/C) and Rick (4:5/AC) played with English-speaking peers in many free-play learning situations. There were also examples that showed Amy (3:6/R), Sarah (3:8/B), Luke (3:3/M), Jim (3:1/M) and Xiaohan (4:8/M) playing alongside English-speaking peers or observing them on free-play occasions. Central to Rogoff's (1998) theory is that children engage with other people through explicit or tacit arrangements, which, for her, indicate an individual's creation of "opportunities to learn through diverse processes of participation" (p.700). In relation to this study, these self-created peer learning experiences could be made as a basis for the children's fulfilment of their interest in English-speaking peers.

The study children's interest in English-speaking peers was more apparent in their reply to my interview question regarding whether they liked playing with English-speaking peers.

Leah $(\mathbf{C P})^{7}$ : $\quad$ I have many friends. I have Chinese friends. I have other friends too.

Amy (CP): I want to play with many other children.

Sarah (NCP) ${ }^{8}$ : I like playing with other children

Rick (CP): $\quad$ I play with Alan (an English speaking child)

Luke (NCP): I like having friends

$\operatorname{Jim}(\mathbf{C P}): \quad$ I play with everyone

In table 7.3 I collated information regarding each child's play experiences with peers during free-play sessions. The information was recorded from my first visit to each child. The purpose of this record was to gain initial information about with whom and how the children tended to play, and how long they played together. The period for each recording was one hour, excluding the child's interactions with any adults or their participation in any teacher-organised experiences.

\footnotetext{
${ }^{7} \mathrm{CP}$ : Chinese peers are available in the centre.

${ }^{8} \mathrm{NCP}$ : Chinese peers are not available in the centre.
} 
Table 7.3: Children's play experiences with peers during an-hour free play session

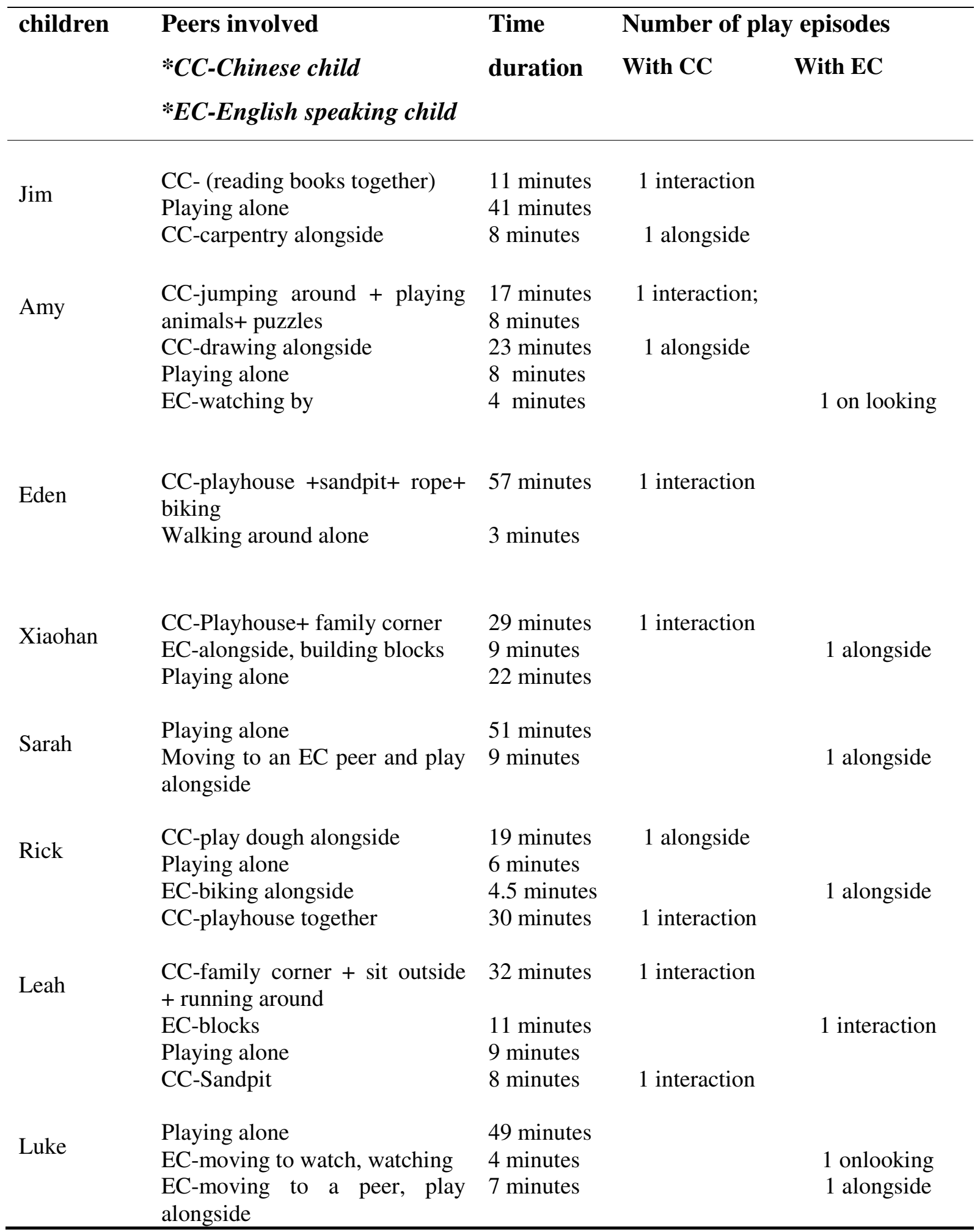


The table indicates that the children were all involved with peers at times. Although the children who had Chinese peers interacted with them, many played with and alongside English-speaking peers or were onlookers. If interests in peers are reflected by "the onlooker, parallel play, involved observation, and initiation measures" (Guralnick, Connor, Hammond, Gottman \& Kinnish, 1996, p.484), then the children demonstrated interests and willingness to be with English-speaking peers.

Corsaro's study on peer culture indicates how onlooking behaviour and being alongside peers are basic means for "creat[ing] a series of embedded peer cultures" (2005, p.44). In my study, the children's interest in English-speaking peers had noticeable effects on their relationships. I observed some children, Leah (4:6/C) and Rick (4:5/AC) in particular, attempting to maintain interactions with English-speaking peers through using appropriate social skills, such as turn taking and sharing. There were also examples when the children, who were rarely observed to interact with Englishspeaking peers, responded to opportunities to play with English-speaking peers after watching them, or playing alongside them for a while.

It was also observed that despite their interest and desire to relate to English-speaking peers, many Chinese immigrant children could not easily do so. They did not appear to have the knowledge of appropriate phrases to respond to opening bids from their English-speaking peers. When some study children did respond to English-speaking peers, they were hesitant, and further development of these contacts appeared to be subject to peer responses to them. On some occasions, the study children had difficulty entering into groups with English-speaking peers and appeared anxious and ineffective communicators. This is consistent with the report by Coplan, Prakash, O'Neil and Armer (2004) who after investigating immigrant children's difficulties in engaging with peers of the predominant cultural group, concluded that these were due to a tension between the children's desire for relationships and their lack of confidence in maintaining them.

The children whose data gave explicit evidence about difficult peer relationships with English-speaking peers are Xiaohan (4.8/M), Jim (3.1/M), Amy (3.6/R), Luke (3.3/M) and Sarah (B). The question then is: Why so? Beyond the simple explanation that these children were not sufficiently competent with the English language (Deegan, 1991; 
Tabors, 1998), it is also possible that the habitual interactions between the Chinesespeaking peers, in the cases of Xiaohan, Amy and Jim, and the high levels of solitary play that Sarah and Luke engaged in due to the limited interactional opportunities they could get from their non-Chinese peers, contributed to them becoming less preferred playmates to their peers. Sarah and Luke, neither of whom had Chinese peers, displayed limited social involvement in sustained peer play, which very likely contributed to them being less skilful social players, thus creating a cycle that consolidated their unwelcome status as playmates for their peers. The communication and social 'incompetence' of the children meant that it was difficult for them to access their English-speaking peers, as peer connections inevitably require certain levels of English and appropriate forms of social behaviour.

In this research, the children's relationships with English-speaking peers can be grouped into four categories. The first category is 'easy peer-relationships', represented by those of Rick and Leah, who were able to communicate in English and knew the centre well as they had been there for two years; in each case I did not find any evidence that they had difficulties in playing with English-speaking peers. The second category is 'minimal peer-relationships', experienced mainly by the children who did not speak English well and who had Chinese-speaking peers in their centres. It is conceivable that the easy relationships with their Chinese-speaking peers led them to prefer the easier option of friendship with them, rather than exerting themselves with English-speaking peers, with whom they were less confident. The third category is 'cautious peerrelationships'. The children in these relationships were Jim, Sarah, Xiaohan, Amy and Eden. They appeared diffident, cautious and hesitant when responding to opportunities to interact with English-speaking peers. The last category is 'difficult peerrelationships', shown predominantly in Sarah's and Luke's data. Because they could not speak English with confidence and did not have Chinese peers in the centres, they faced difficulties with their peer relationships. These categories are presented in Table 7.4 
Table 7.4: Categories of the children's relationships with English-speaking peers

\begin{tabular}{|c|c|c|c|c|}
\hline $\begin{array}{l}\text { Categories of } \\
\text { relationships }\end{array}$ & Children & English abilities & $\begin{array}{l}\text { Length of centre } \\
\text { attendance }\end{array}$ & $\begin{array}{l}\text { Chinese peers } \\
\text { in the centre }\end{array}$ \\
\hline Easy & $\begin{array}{l}\text { Leah } \\
\text { Rick }\end{array}$ & $\begin{array}{c}\text { Competent } \\
\text { Almost competent }\end{array}$ & $\begin{array}{l}2 \text { years } 4 \text { months } \\
2 \text { years } 5 \text { months }\end{array}$ & $\begin{array}{l}\text { Yes } \\
\text { Yes }\end{array}$ \\
\hline Minimal & $\begin{array}{l}\text { Amy } \\
\text { Xiaohan } \\
\text { Jim } \\
\text { Eden }\end{array}$ & $\begin{array}{c}\text { Routine conversations } \\
\text { Minimal } \\
\text { Minimal } \\
\text { Minimal }\end{array}$ & $\begin{array}{l}4 \text { months } \\
2 \text { months } \\
1 \text { month } \\
3 \text { months }\end{array}$ & $\begin{array}{l}\text { Yes } \\
\text { Yes } \\
\text { Yes } \\
\text { Yes }\end{array}$ \\
\hline Cautious & $\begin{array}{l}\text { Sarah } \\
\text { Amy } \\
\text { Xiaohan } \\
\text { Jim }\end{array}$ & $\begin{array}{c}\text { Basic conversations } \\
\text { Routine conversations } \\
\text { Minimal } \\
\text { Minimal }\end{array}$ & $\begin{array}{l}10 \text { months } \\
4 \text { months } \\
2 \text { months } \\
1 \text { month }\end{array}$ & $\begin{array}{l}\text { No } \\
\text { Yes } \\
\text { Yes } \\
\text { Yes }\end{array}$ \\
\hline Difficult & $\begin{array}{l}\text { Luke } \\
\text { Sarah }\end{array}$ & $\begin{array}{c}\text { Minimal } \\
\text { Basic conversations }\end{array}$ & $\begin{array}{l}2 \text { months } \\
10 \text { months }\end{array}$ & $\begin{array}{l}\text { No } \\
\text { No }\end{array}$ \\
\hline
\end{tabular}

As shown in this table, the children who developed easy relationships with Englishspeaking peers had three types of cultural tools available to them: the English language which they could use well; knowledge about the centre after being there for more than two years; and Chinese-speaking peers in their centres. The difficult peer relationships between Luke and Sarah and their English-speaking peers were the result of the inaccessibility of nearly all these cultural tools. The children whose relationships with English-speaking peers were categorised as 'minimal' or 'cautious', had at least one cultural tool absent.

This table indicates that the children who did not have easy relationships with Englishspeaking children had all attended the centre for less than a year and perhaps because of this, had developed limited knowledge about the centres. This shows that the duration of the study children's attendance in their centres influenced their learning experiences. However, it is still uncertain whether this finding can be applicable in Luke and Sarah's situation. If Leah and Rick developed their English skills and knowledge about the centres because "Rick had Chinese peers to help him when he first started" (Rick's teacher Jenny) and "the attendance of Chinese children is always high at our centre" (Leah's teacher, Carol), then Luke and Sarah, who were not in such a learning context 
might not be able to achieve the same learning outcomes. Sarah had 'basic' English ability, but this might have developed with her sister at home (see section 6.3.1.2.7).

The following two sections focus on the last two types of peer relationships between the children and their English-speaking peers. Unlike the 'easy' and 'minimal' peer relationship categories, it is very likely that the children in 'cautious' and 'difficult' peer relationships demonstrated behaviours and experienced incidents particularly significant for their learning experiences in an unfamiliar cultural community.

\subsubsection{Relating to English-speaking peers with caution}

The following are examples that illustrate two children contacting peers in cautious and tentative ways.

Jim (3:1/M) walks to an English-speaking boy who is playing chess on the floor. Jim sits down by the boy, watching. He then moves to the chess box, taking a piece out and adding it to the boy's chess board. The boy ignores Jim's proffered chess piece and carries on with his own play. Jim adds one more piece. The boy turns to him: "ok". Jim quickly puts another piece on. $\left(\mathrm{Jim} / 4^{\text {th }}\right.$ visit, 11:31am)

Sarah shows a similar behaviour as follows:

Sarah (3:8/B) walks to the swings. She watches a child on one of the swings and smiles at the child. The child smiles back. Sarah then moves to the child's back and gives a small push to the child's swing. Sarah then moves to the front to see the child's face. The child says: "push me". Sarah moves back and pushes the child. (Sarah $/ 5^{\text {th }}$ visit, 9:21am)

These two children's initial contacts with their peers were uncertain and cautious, but once assured by the other child, they moved further. Their behaviours support Igoa's view that young second-language learners are keen to blend with other children but "their emotions and fears hold them back" (1995, p.38). It is likely that the children were not confident in pursuing the peer experiences because they were fearful of peer disapproval, so they were careful to check out the effect of their tentative actions with the peers before they took further action.

In this study, the relationships between the children and their English-speaking peers appeared to be largely determined by peer attitudes and responses to them. As shown in 
the examples above and those that follow, if the peers were friendly, interested in the Chinese immigrant children, or took initiatives, mutual interactions could emerge:

Eden $(3: 3 / \mathrm{M})$ is sitting on the floor, watching some children doing figure play. An English-speaking boy sits close to him. Eden moves back a bit to allow more space between him and the boy. The boy moves closer to Eden, facing him and smiling at him. Eden then reaches his hand to the boy's face and smiles back at the boy. The boy goes to touch Eden's face. (Eden/2 $2^{\text {nd }}$ visit, 11:58am)

Xiaohan $(4: 8 / \mathrm{M})$ goes to the family corner. She sits on the floor, picking up a toy monkey. Two girls come in and sit on the bed near Xiaohan. One girl lies down, pretending to be a baby. The other girl feeds her with a 'bottle'. Xiaohan turns her head to the girls, watching. When the girl who is feeding turns around, she sees Xiaohan and hands the 'bottle' to Xiaohan. Xiaohan stands up, goes to feed 'the baby'. She even gently pats her saying 'baby'. (Xiaohan $/ 4^{\text {th }}$ visit, 3:22pm)

The behaviours demonstrated by Eden and Xiaohan were similar to those of Jim and Sarah and reflected their caution and diffidence. However, once assured that their advances would not be rejected by the English-speaking peers, the study children furthered their actions. Therefore, when the responses were friendly and the Englishspeaking peers displayed clear attempts to include them, the Chinese immigrant children interacted with these peers. This is especially clear in Eden's case, as he moved away from the English-speaking peer, possibly displaying uncertainty about whether the peer would like to sit close to him. However, when the peer moved closer to Eden and smiled at him, Eden's hesitancy disappeared. The smile would have indicated to Eden that the boy was well disposed to him and Eden then took the opportunity to touch the boy. In Xiaohan's example, the peers included her in the play so Xiaohan accepted the opportunity to join them in play.

All the four examples shown above can be interpreted as evidence that the friendly and approachable attitudes of English-speaking peers to the study children made a difference to the children's social behaviours, which is consistent with the findings of previous research (Guralnick, et al, 1996; Kemple, 2004). According to Suarez-Orozco and Suarez-Orozco (2000), "the interpersonal tone set by peers is an important variable in the adaptation of immigrant children" (p.24). Based on these examples, it is possible to infer that if English-speaking peers were unfriendly and did not clearly include the children, then the children would face difficulties in relating to those peers. Two examples below illustrate these difficulties. 
In the following example, Amy (3:6/R) seemed to avoid approaching English-speaking peers because they did not appear friendly and did not include her in their group.

Amy is drawing on a sheet of paper alongside three English-speaking girls. She is sitting on one side of the table next to Iby (teacher) and the three English-speaking girls are sitting next to each other on the other side of the table. The English-speaking girls are drawing and they sometimes giggle among themselves. Amy colours a picture all red while the other children use a range of colours to draw. Amy stops, looking into the pen holder which is empty as the other pens have been taken out by the girls and placed next to them.

Iby: "finished?".

Amy: "no", shaking head.

Iby: "then you need to tell them you need pens".

Amy makes no move. Iby gets a couple of pens from the other girls and hands them to Amy. Amy continues drawing. (Amy/2 ${ }^{\text {nd }}$ visit, 2:03pm)

It was obvious that Amy wanted the pens, but she did not approach the other girls for them, perhaps because, as she told me after the incident "I dare not ask", thus indicating that she was not confident to approach the other girls. In view of Amy's social behaviours here, it is possible to infer that Amy's lack of confidence in asserting her wish for the pens with the English-speaking girls, indicates awareness on Amy's part that she was not a member of that social group, and did not feel that her approach to the girls would be met with friendly compliance, but rather she would risk rejection. Another reason could be that with Iby around, Amy relied on Iby to help her. I do not interpret the cause of this to be Amy's language limitation, because while her English was not proficient, Amy knew enough to handle the situation.

In this next example, Sarah (3:8/B), likewise, appeared afraid of getting near to her English-speaking peers:

Sarah walks to the seesaw where two children are playing. Nearby her teacher Anne is sitting with some other children on a bench. Anne: "Sarah, come to sit here and wait", pointing to a space by a girl on the bench. Sarah walks towards Anne. She stops by the bench, glances at the space and sits down. She keeps a big gap between her and the girl already sitting there. The girl turns her head to Sarah, gives her a glance and then moves her head back to face the seesaw. Sarah stays where she is. (Sarah/2 ${ }^{\text {nd }}$ visit, 11:23am)

Sarah kept that gap from her peers throughout her waiting time by the seesaw, and she appeared unsure whether she was welcome. Similar to Amy's situation above, Sarah's lack of group status could be responsible for her reserved behaviour, as it was unlikely 
that she wanted to be separated from her peers. Rather, Sarah did not know if the other children would include her, similar to a case described by Igoa (1995). This finding again highlights the importance of peer attitudes for the children's social engagements. If Amy and Sarah's peers had smiled at them or approached them as the Englishspeaking children did with the children in the examples earlier in this section, they might have joined the peers in interactive ways. Alternatively, it is possible that if the peers had been Chinese, the two children might have approached or joined them. The above examples demonstrate that refraining from social contacts probably due to the children's lack of ability to relate with peers of a different cultural community (Chang et al., 2005). With reference to Leah and Rick's easy interactions with their Englishspeaking peers, Sarah and Amy also needed to speak English well and to have sufficient knowledge about their centres. However, Eden (3:3/M), Jim (3:1/M), Xiaohan (4:8/M) and even Sarah (3:8/B) herself had succeeded in interacting with their English-speaking peers as pointed out earlier. What happened here to Sarah (3.8/B) and Amy (3.6/R), therefore, was very likely to be result of their reading of the peers' cues and their interpretations that the English-speaking peers did not wish to include them. In comparison with the children's easy initiation of relationships with their Chinesespeaking peers, for example between Amy and Ken, the children were cautious with English-speaking peers, possibly because they were aware of their 'different' identities. In this analysis, the learning experiences discussed here strengthens the impression that similarity was a key driving force for the children's development of peer relationships, especially for those who were new to the EC centre. Moreover, this finding indicates that despite the three important tools employed by the study children to develop relationships with English-speaking peers as shown in Table 7.4, the attitudes of English-speaking peers in return were a significant mediator for the Chinese immigrant children's involvement in groups.

\subsubsection{Aggressive reactions to peer conflicts}

Three children, Xiaohan, Saran and Luke demonstrated aggressive behaviour when dealing with conflict with English-speaking peers. One reason for the aggression appeared to be the need to assert their wishes. The children used physical means when feeling that the undertaking of a personal action was threatened (Persson, 2005). The following are two examples: 
Sarah runs to a swing when a child leaves it. Another girl is running to it too. Sarah gets to it first and jumps onto it. When the other girl gets there, she tries to prise Sarah's hand away from the swing. Sarah keeps her hands on the swing. The girl shouts: "you off, off". Sarah jumps off the swing, holding the girl's hand and hits her. (Sarah/ $3^{\text {rd }}$ visit, $11: 27 \mathrm{am})$

Xiaohan, as shown in the note below, hits a peer too.

Xiaohan plays on the ground near the playhouse. She walks to a plank that is almost connected to the playhouse, lifts it up and pushes it towards the playhouse, tyring to connect it further to the playhouse. An English-speaking boy comes out of the playhouse, pushing the plank out, seemingly wanting to jump out of the playhouse. As soon as the boy touches the plank, Xiaohan hits the hand of this child. (Xiaohan $/ 4^{\text {th }}$ visit $3: 19 \mathrm{pm})$

Sarah and Xiaohan attacked their peers probably because the peers snatched what they had got first. Although Xiaohan had used appropriate communication strategies, such as negotiation or compromise in conflict situations with her Chinese-speaking peers, her response to the conflict with this English-speaking peer was physically aggressive. She made no attempt to resolve the problem in a more appropriate way. Xiaohan and Sarah had not been observed to play with these peers previously, and the peers did not appear friendly towards them, so they might have seen these peers as a threat with whom they could not verbally communicate effectively to solve the problems. Hitting was therefore a way of communicating their wish to stop the peers in their actions. In the previous section I argued that the children were cautious or they minimised social contacts with unfamiliar English-speaking peers. For many children, including Xiaohan and Sarah, there were no records of their initiating a peer contact that led them to a conflict situation. For this reason, their aggressive actions towards the English-speaking peers appeared to be in response to the peers' behaviours towards them.

Luke's experiences with English-speaking peers, however, were different. He made many attempts to initiate contacts with other children and when these were rejected, he took aggressive actions:

Luke walks around. He walks to an English-speaking girl and gives her cuddle. The girl pushes him away. Luke hits the girl in the face. (Luke/2 ${ }^{\text {nd }}$ visit, 11:21am)

An English-speaking girl is sitting on a cushion to read a book. Luke walks to her, bends down to the girl, giving her a smile and sits down by the girl. The girl quickly stands up and leaves. Luke watches her leave, then stands up, chasing the girl. He gets to the girl and snatches the book from her hand: "gei wo" [give it to me]. The girl hits 
him, but Luke grabs the book and runs away with it. On the way, Luke drops the book. The girl picks it up. Luke turns back to her and snatches it again from the girl. The girl yells, but Luke runs away with it. (Luke/ $3^{\text {rd }}$ visit, 9:21am)

In both instances, it is evident that Luke was disappointed that his friendly initiative was rejected. In the second example, he did not appear to have approached the girl because he was after the book, but rather her acceptance of him. In these two situations, Luke tried to relate to peers but was rejected. Luke could have been angry, which seems to have motivated him to attack the peers.

Luke repeated this behaviour in the following example:

Luke stands by two boys who are putting together some puzzles. His teacher Nicole is making lunch at the kitchen and other teachers are elsewhere. A boy stands up from his puzzle, looking for a piece. Luke walks to the puzzle shelf and finds it. He hands the piece to the boy, who does not take it. Luke gives him a nudge with the piece. The boy ignores him. Luke throws the puzzle piece at the boy's face. (Luke/ $4^{\text {th }}$ visit, 11:30am)

In this situation, Luke meant to help, but the peer's neglect of his intention frustrated him.

Luke's attempts to help peers as a means to become included were obvious in his data. I noted him standing on a chair, fetching a paint brush and handing it to a peer who had just joined the painting table with Luke and other children. Disappointingly, the peers did not acknowledge Luke's attempt to help and in this case, the peer just took the paint and sat down. There is no doubt that Luke was disappointed, which then rose to the level of anger so that he attacked his peers. In addition to hitting peers, Luke sometimes released his anger by throwing toys around or destroying other children's work. His behaviours bear out previous research which has demonstrated that children with low peer acceptance are prone to displaying negative social actions (Malcolm et al., 2006). If Luke's peers were as friendly or approachable as the peers in section 7.2.2, he might have reacted differently in these situations.

Unfortunately, as Denham, Mason, Caverly, Schmidt, Hackney, Caswell and DeMulder (2001) have shown, children's aggressive behaviours often result in and further strengthen their low social status in groups. They noted that "preschoolers, who show 
negative affect, particularly anger, are often seen by both teachers and peers as troublesome and difficult. Specifically, angrier preschoolers are reported as disliked by their peers" (p.291). During my study with Luke, I was told by an English-speaking boy that he did not like Luke because Luke was naughty. Tabors (1998) could have understood this issue in his term of "double bind" (p.22) and explained Luke's social difficulties to be the result of his language incompetence. Luke was caught in a vicious cycle with the slow progression in language leading to the slow development of social skills. Sadly, even when the study was complete, I still did not see Luke forming peer relationships with any child in his centre. So it is predictable that Luke would continue to struggle with his peer relationships unless he received specific support.

The evidence concerning the study children's 'cautious' and 'difficult' relationships with English-speaking peers suggests that these relationships operate with very complex dynamics. The processes and outcomes are influenced not only by the English language and their knowledge of the centres, but also the children's emotions and their perceptions of their own status, their peer status, and peer attitudes towards them (Hruska, 2000; Igoa, 1995; Krupa-Kwiatkowski, 1998). While these dimensions were individually and contextually specific, they were interdependent and nested within each other, in the larger context of the sociocultural community of the EC centres.

From the examples of the study children who had Chinese peers in their centres and used Chinese cultural tools, it would appear that the reason why some children were cautious or applied inappropriate strategies with the English-speaking peers might be their awareness of the inapplicability of their family cultural tools. In those situations, the children's established abilities or their family cultural knowledge or practice, particularly in relation to social interactions had to be positioned away from those of the EC centres. If "children seek connections between old and new situations" (Rogoff et al., 1993, p.235), the above examples that illustrate the children's difficulty in relating to English-speaking peers have important implications for the reconciliation of the different cultures in their peer relationships. 


\subsection{Chapter summary}

The question addressed in this chapter was how peer relationships mediated the Chinese immigrant children's learning experiences in their EC centres. Two positive answers to this question were that:

(i) the children were all interested in peer relationships; and

(ii) regular and frequent interactions with peers acted as a strong basis for the evolution of the children's group membership.

The findings in this chapter indicate that the sociocultural theories of Vygotsky (1978) and Rogoff (2003), particularly their concepts of peer support and guided participation, as well as Corsaro's (2005) notion of a peer culture with a strong focus on shared play, all provide useful theoretical tools for explaining the importance of peer relationships in children's learning and development. The idea of learning as a process of developing identity, meaning and social practices as captured in the concept of 'community of practice' (Wenger, 1998) illuminates the purposes and outcomes of some the children's learning experiences with their Chinese-speaking peers.

In this chapter I built on the discussion in Chapters 5 and 6 regarding the relationship between Chinese immigrant family culture and the mainstream culture of New Zealand EC centres in the contexts of the children's peer relationships. The data in this chapter suggest that both cultures influenced the development and maintenance of peer relationships for the children. Firstly, all the children, regardless of their English language abilities, attempted to deal with their social worlds by relating to Chinese peers when they were available, and seeking and creating opportunities to use their family culture, particularly the language and social choices. Secondly, the children who were knowledgeable about the centre programmes and were competent and confident in English developed easy relationships with both Chinese-speaking and English-speaking peers, thus showing an ability to connect two types of peer-mediated cultural knowledge in their learning and development. Thirdly, when they recognised the value of their family culture to create familiar learning experiences for them and their Chinese peers, the children sometimes chose to keep their family culture aside from the mainstream culture of New Zealand EC centres. Lastly, there were occasions when the children 
gave up on their family tools, possibly due to their awareness that their tools were different from the cultural tools of the people who shared their culture.

The different influences of the two cultures on the children's peer relationships were visible in this research. In particular, peer relationships appeared to be formed in specific language and cultural contexts, occurring in sameness-oriented ways, and operated more efficiently when the children related with Chinese peers. This finding is consistent with those of other researchers (Chen et al., 1992; Kantor et al., 1998; Suarez-Orozco \& Suarez-Orozco, 2000). Despite their interests in playing with all their peers and the competencies of some Chinese children in relating to English-speaking peers, the children who had Chinese peers all came to see themselves as part of the Chinese peer group. Peers within Chinese immigrant cultural groups guided each other's learning by interacting in ways that appeared able to meet their needs. In contrast, the learning experiences of children with English-speaking peers were complex; there were cases in which the children could not access or develop learning experiences with English-speaking peers because the English-speaking peers did not appear friendly or approachable, and the children could not communicate effectively with them. Underlying the children's relationships with English-speaking peers is also the difficulty for some of them to build confidence to approach their English-speaking peers and enter into relationships with them. The study illustrates that the process and outcome of peer experiences between the Chinese immigrant children and their Englishspeaking peers relied to a large extent on the children's English proficiency, their knowledge about the centres, as well as the attitudes of English-speaking peers towards them. The many features of peer relationships, observed in this study, illustrate the processes in which the children, when engaging in peer experiences and developing group membership, addressed issues related to similarity, difference, self, others, communication, and peer attitudes.

With respect to their development of membership status or a sense of belonging in the learning settings, the contrast between the children who had Chinese peers and the two children, who did not, is striking. The children who had Chinese peers in their centres built their sense of belonging there, while those who did not have Chinese peers available in their centres experienced difficulties settling. The value of their family 
cultural tools in mediating the peer learning experiences of the Chinese immigrant children in English-speaking EC centres is a key finding in this research.

The sociocultural perspectives that have framed the analysis in this chapter reveal the study children's social competence as situational and affected by a dynamic synthesis of confidence, strategies, communication abilities, and knowledge and skills to use appropriate cultural tools. Examples of this came from Leah and Rick's successful formation of relationships with peers of both Chinese and English-speaking backgrounds. The children's easy relationships with their Chinese peers also provide such evidence. It appears here that although Sarah and Luke seemed to have lacked social competence, this might have been different if they had had peers of the Chinese cultural group in the centres with whom they could communicate, and use common cultural tools to explore the new cultural knowledge. If the establishment of peer relationships entails the application of verbal communication (Chang et al., 2005) and "locally constructed cultural knowledge held by a group of peer players" (Kantor et al., 1998, p.148), it is understandably difficult for Sarah or Luke to understand and access the cultural knowledge of a community in which they could not easily communicate. An implication of the children's behaviours in peer situations therefore is that immigrant children need support to access the cultural knowledge of the mainstream peer players.

The chapter that follows brings together the main findings of this research, conceptualises the learning experiences of the children from an overall perspective and draws out their educational implications. 


\section{Chapter 8: Drawing the findings together and looking forward}

\subsection{Introduction}

This chapter brings together the main findings of my research to provide an overall answer to the two central questions of this research:

1. How do Chinese immigrant children experience learning and development in New Zealand early childhood centres?

2. How can Chinese immigrant children be supported towards a positive learning experience in New Zealand early childhood centres?

Based on the findings in Chapters 5, 6 and 7, this chapter considers how the study children showed a pattern of learning experiences in New Zealand EC centres through using language, the key cultural tool, via the mediation of their relationships with their peers and teachers. It concludes with suggestions for specific educational strategies in response to the learning experiences of the children.

\subsection{Drawing together: Conceptualizing the children's learning experiences}

The ideas that informed my thinking throughout this research derived from the sociocultural perspectives on learning and development initially formulated by Vygotsky (1978) and expanded by others, particularly Rogoff (2003), Russell (2002) and Wenger (1998). My theoretical starting point was to recognise that the children's learning experiences are socioculturally mediated and that each child is a nexus of social and cultural relations. This view informed the analysis and understanding of the findings of this research.

The New Zealand ECE curriculum document, Te Whāriki (Ministry of Education, 1996) states that EC centres are sociocultural contexts that consist of multiple and diverse ways of being, in which congruence and difference in cultural practices and beliefs occur and interact. There are many interconnected subcultures within the centres, such 
as the personal cultures of teachers and individual children. Chinese immigrant family culture and the mainstream culture of EC centres are part of the wider sociocultural contexts.

This research explored how eight Chinese immigrant children experienced the sociocultural contexts of their New Zealand EC centres and in particular how the two cultures, namely the Chinese immigrant family culture and the mainstream culture of New Zealand EC centres, mediated the children's learning experiences. The mediation of the children's learning through two cultural communities, with their own sets of tools is, to my mind, at the heart of this research. The focus on the two sources of cultural tools means that this research is intercultural in nature because it is concerned with the bringing together of two cultures within a specific research setting (Mulder, Rance, Suarez \& Condori, 2000). The analytical discussion of my findings thus is an integration of empirically-derived intercultural knowledge with theoretically-inspired sociocultural concepts. Because of this, interpretations of the learning experiences of the study children depend on an understanding of how the two differing cultures were related. In other words, this study was to identify the possibilities of intercultural relationships.

On the basis of the sociocultural and intercultural considerations discussed so far, two key conclusions may be drawn about the learning experiences of the children in New Zealand EC centres:

(i) the learning experiences of the children relied on the application of cultural tools from both Chinese immigrant families and New Zealand EC centres.

(ii) the learning experiences of the children were driven by children's drive to belong.

Figures 8.1 and 8.2 illustrate these findings, which are discussed in detail in subsequent sections of the chapter. 


\subsubsection{Learning experiences of Chinese immigrant children drew on the application of cultural tools of Chinese immigrant families and those of New Zealand early childhood centres}

The findings of my research converge around the conclusion that the learning experiences of the Chinese immigrant children were the result of a process in which they located familiar cultural mediators from their families (e.g., Chinese peers or Chinese-speaking teachers) within their EC centres, applied family cultural tools (e.g., Chinese language, social choices, parental beliefs and practices, learning attitudes), and associated them with the mainstream cultural tools of New Zealand EC centres (e.g. English language, teaching beliefs, learning programmes). When the children used the Chinese language to think and talk about their learning experiences in the centres, actively sought togetherness with Chinese people, and had high regard for teachers, they located and used family learning experiences. With these practices, the children also demonstrated a determination to learn about the new cultural tools with their family cultural tools.

The children's engagement in experiences with the cultural tools of the EC centres was similarly noticeable through, for example, their use of the English language, active explorations of the centre programmes, interactions with or playing alongside Englishspeaking peers, and close following of teachers. This finding can be interpreted through Vygotsky's point that "humans function in a range of sociocultural contexts and are hence required to carry out a range of cultural activities" (Wertsch, Tulviste \& Hagstrom, 1993, p.352). Wenger's (1998) concept of boundary crossing provides a useful way of thinking about the children's learning as a distributed process of creating new activities and expanding their involvement in cultural tools, rules and communities. Rogoff and her co-researchers' term of hybridity is also relevant here (Rogoff et al., 2007). By hybridity, they mean a process where children achieve specific purposes through "using particular interactional formats as cultural tools". In this research, the children were looking for ways to negotiate the co-existence of two sets of cultural tools to function, learn and develop in intercultural learning contexts. Within these contexts, the children entered into a culture-culture relation through which they created their own repertories of practice. 
This research demonstrated a range of possible relationships between the cultural tools of Chinese immigrant families, and those of New Zealand EC centres. The children's preference for particular cultural engagements, the availability of Chinese peers and teachers, as well as the centre's learning programmes and teaching beliefs, all contributed to how their family cultural tools were used by the children in their centres. The Chinese immigrant family culture thus became related to the mainstream culture of New Zealand EC centres, and created a site where it mediated the children's learning experiences with the mainstream culture of the centres. The possibilities for these intercultural relationships depended on the children's ability to access either or both sets of cultural tools.

Through the children's use of their family cultural tools, we saw these tools

(i) bridge the children's involvement with the cultural learning practices of their centres

(ii) converge with the cultural tools of the EC centres,

(iii) kept aside as an independent set of learning mediators, and 4) kept away from the culture of the EC centres.

This range of intercultural relations influences the relative position and role of the Chinese immigrant family culture in the children's sociocultural context. As noted in Chapter 2, by the 'sociocultural context', I mean the environmental influences on children's learning and development that include people's beliefs, practices, languages, and relationships in the EC centres, where both Chinese immigrant families and the mainstream teaching and learning programmes of the centres, play a part.

In figure 8.1, the intercultural possibilities between the two cultures in the children's sociocultural context are depicted. While they appear simple in this illustration, as will be shown later in this chapter, the actual relations between the two cultures were complex, requiring an analysis of the driving forces for these relations. 


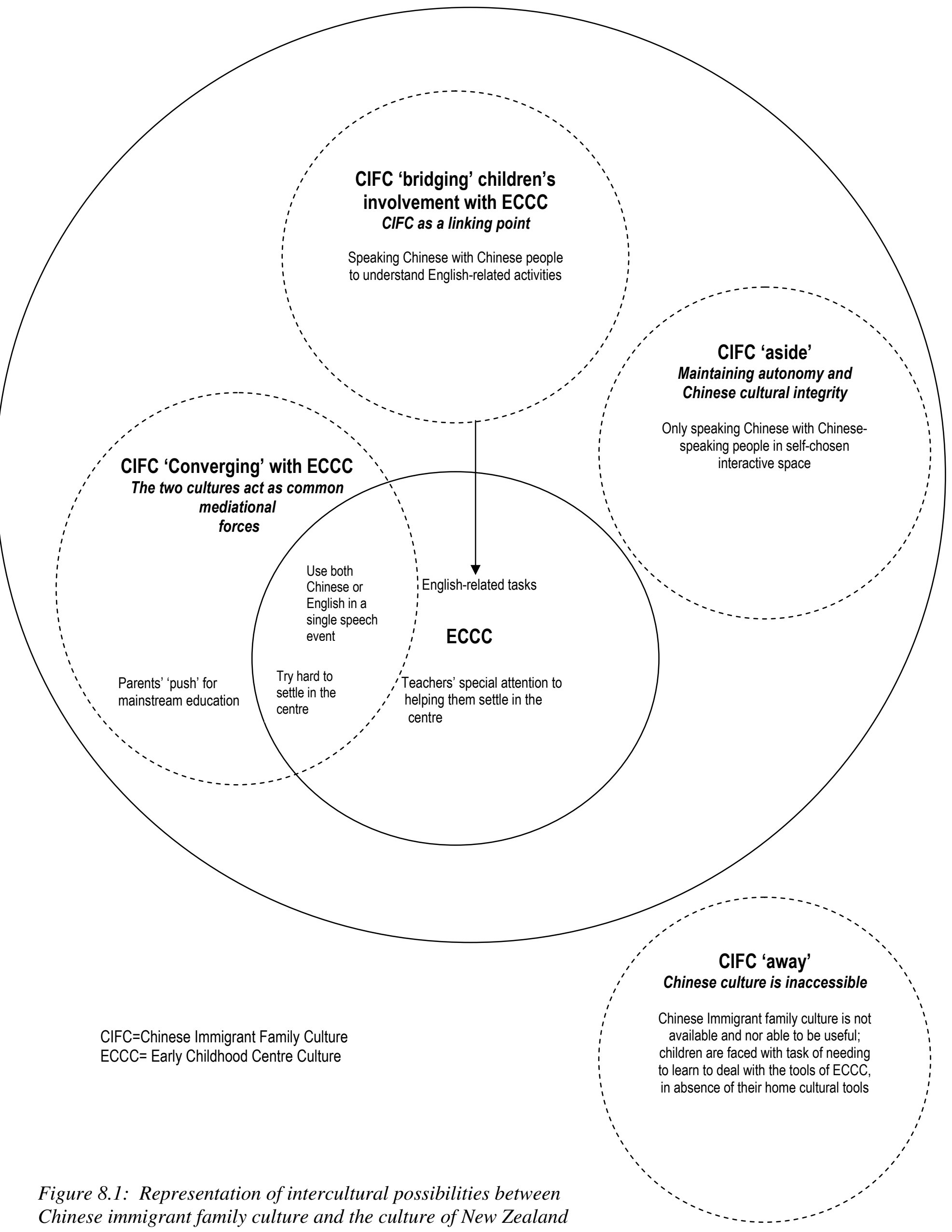

Chinese immigrant family culture and the culture of New Zealand 
The first intercultural possibility between the Chinese immigrant family culture and that of New Zealand EC centres, was the children's use of their family cultural tools to bridge their own involvement with those of the centres. A child's request in Chinese made to Chinese peers or teachers to understand an English-related activity was an example of this. Underpinning the relationship between these two cultures was the children's recognition that Chinese language and Chinese people were useful for their learning of the other cultural knowledge. When Chinese immigrant family culture acted as a bridge in the children's involvement with the centre's cultural tools, cultural practices such as speaking Chinese in private situations, or with Chinese peers to learn about the new cultural practices, created a linkage between what the children knew and what they did not know (see figures 6.2 and 7.2).

According to Radice (2008) the idea of communication and connection forms the essence of the bridge as metaphor. The nature of a bridge as providing a way across and a way of connection, as defined by Schwarzweller and Brown (1962), also fits well with the notion of culture-as-bridge. Thus, the use of Chinese immigrant family culture as a bridge describes some of the children's learning experiences and captures the process of their development as one in which known cultural practices were able to communicate and thus connect with unknown ones. The emphasis on family culture as a bridge highlights that while Chinese immigrant family culture was different from that of New Zealand EC centres, certain practices of Chinese immigrant families could bridge the gap. Trumbull, Rothstein-Fisch, Greenfield and Quiroz (2001) make the point that the view from a bridge is enjoyable because one can see two sides almost simultaneously.

Furthermore, by actively participating in both cultural activities, the children demonstrated an ability to operate within a convergence of the two cultures. The way in which the children interchangeably used two languages alerted me to the fact that the children's practices within New Zealand EC centres were converging with certain features of the cultural practices of Chinese immigrant families (see figure 6.1). In addition, the children tried hard to settle into the centres, because their parents' encouragement for them to develop 'Kiwi' knowledge, worked together with their teachers' focus on helping them to settle, and the two cultures exerted a common mediational force on the children's actions (see figure 5.1). Jenkins (2006) has argued that cultural convergence indicates the intersection between the new and old cultures. 
Other important findings were the examples of Chinese immigrant family culture being kept apart from the culture of the centres. The use of the Chinese language among Chinese children to interact with each other in their own interactive spaces is a good example of the children maintaining their family culture as something independent from the overall culture of the centres (see figure 7.1). Serpell (1993) understood this type of interaction to be:

...the children taking on cognitive authority, demonstrating confidence and competence to act autonomously and also develop a sense of membership in the group and corresponding ownership in its cultural resources. The authority of this claim 'this is my language, my culture, my community' is simultaneously based in a sense of belonging (of being owned and accepted by the group). (p.362)

This statement is profoundly important and highlights the power of a familiar culture to mediate children's learning experience in an unfamiliar cultural community. Based on this statement, one can see that it was not that the children used the cultural tools of their families in a non-Chinese cultural community to assert that Chinese was their family culture. Rather, their family culture provided the children with the most fundamental tools through which their established sense of belonging in families could be kept intact and undisrupted in a setting where they otherwise could not easily do so. My argument, then, is that the children's 'sense of belonging' was an essential factor within their application of particular cultural tools to mediate their learning experiences.

Key to all the above-mentioned cultural relations was the availability of Chinese peers and Chinese teachers. The way that Chinese immigrant family culture mediated the learning experiences of the children through the Chinese peers and teachers was perhaps most apparent when one considered the experiences of the two children, Luke and Sarah, who did not have accessible Chinese support in their learning settings. The analysis of these two children's learning experiences revealed that the cultural tools of Chinese immigrant families were mostly inaccessible to them because there was nobody in their centres with whom they could use these tools. For example, Luke's teachers did not respond to him appropriately when he made requests to them in Chinese. This may have led Luke to believe that the Chinese language was not useful, and therefore to be kept away from the centre. 
In Chapters 6 and 7, evidence was provided that Luke and Sarah encountered many challenges in their interactions with peers in their centres. As with the other children they undoubtedly possessed many practical cultural tools from their families, and they would have probably tried to locate them in the centres. This can be indicated by them speaking to me in Chinese, their close following of teacher instructions, and their active exploration in the settings. But, in the absence of Chinese peers and Chinese teachers in their centres, there was no means through which their family culture could effectively assist these two Chinese immigrant children's experiences. Instead, the experiences of these two children reveal that the children's possession of cultural tools of Chinese immigrant families (e.g., language and social choices) is not a guarantee that they will be usefully deployed to facilitate learning in a new context. To be effective, the tools need to be used for a productive reason. My data show that although Sarah and Luke had their family cultural tools available to them, their use was not mediated by the presence of others who could use them. As Vygotsky (1932, as cited in Ivic, 1989) argued, it is only through the mediation of people and social interactions that a child uses tools. If we accept that Chinese peers and teachers were significant mediators between the family culture and the new culture of the centres for the study children, then it is possible to understand why Luke and Sarah, with no family cultural mediators, were unable to use these tools to assist them in their EC centres.

The two very different experiences encountered, on the one hand by the study children who visibly used their family cultural tools in their centres and on the other hand by Luke and Sarah who did not, further highlight the importance of family culture to mediate children's learning in non-family cultural communities. Based on Vygotsky's understanding of "culture as something that comes into concrete existence in social processes" (Daniels, 1996, p. 61), the children's gradual involvement in the routines, activities and practices of the centres can be seen to be mediated by their active application of tools from the family culture, which in turn contribute to their ability to make sense of the social processes in the new cultural communities. Rogoff's notions of guided participation and the process of transformation of participation are also illustrated in this study. Through the use of family cultural tools with people who could speak their family language, there was evidence that the children "develop[ed] through participation in an activity, changing to be involved in the situation at hand in ways that 
contribute both to the ongoing event and to [their] preparation for involvement in other similar events" (Rogoff, 2003, p.254).

The transferability of the two key cultural contexts, or the 'activity systems' of the children (Engeström, 1987; Russel, 2002), namely the Chinese family culture and that of the EC centres, are shown to have lent themselves to dynamic, evolving, and distributed learning experiences for the children. These experiences have involved people and cultural tools that afforded or constrained the children's actions. In the context of sociocultural theories of development, the learning experiences of the children can be characterised as a process whereby the children interacted or coordinated the activity systems in their families and in the EC centres (Wenger, 1998). Perhaps as a result, the Chinese language, an identifiable 'boundary object' that the children used to bridge their two different activity systems, became both affordance and constraint in their efforts to cross the boundary zones (see section 6.4 for examples of Eden's 'Chinese-mostly' behaviours).

\subsubsection{Learning experiences of the children were driven by children's drive to belong}

As mentioned above and throughout the findings chapters, the children's motivation to settle in the centres is a central theme in the research. To a certain extent, the children purposefully and strategically created their own learning experiences through the use of appropriate cultural mediators and tools. This argument was theoretically established in Vygotsky's contemplation of the abilities of human beings to structure social interactions "to generate new practices and ideas for inclusion in the culture" (as cited in Serpell, 1993, p.358). Several other lines of sociocultural scholarship also contributed to the discussion of children's competence in creating their own development. Wertch (1991) attributed this to the children's plans to achieve their own goals "through decisions among alternative courses of action, choosing means that have the promise of being successful in the given situation and applying them in a suitable manner" (p.9). Wenger (1998, p.15) suggested that the experiences of children should be explained in terms of their development of "'own' meanings" of participation in their communities of practice. Rogoff et al. (2007) developed the concept of "repertoire of practice" (p.491) 
to focus on "children's own agency in selecting, rejecting, and transforming multiple ways of engaging in the world".

In this research, I found that most of the children effectively used the tools from both cultural communities to produce a new repertoire of practice through

(i) actively exploring in their learning settings;

(ii) building togetherness with Chinese-speaking peers and teachers;

(iii) applying family cultural knowledge and experiences;

(iv) developing and using new cultural knowledge;

(v) creating communicative means;

(vi) engaging in experiences with English-speaking peers and teachers;

(vii) remembering specific happenings; and

(viii) producing inventive devices.

An overall finding of this research is that regardless of the lengths of attendance in the centres, the ages and standards of English competence, all the children were actively and strategically participating in their new cultural community. In addition, it is also apparent that the children held opinions about their own learning experiences and were able to articulate responses when prompted. In other words, the children's own intentions and the consciousness of their actions appeared to be powerful sources of information for building an understanding of the phenomenon of their learning experiences.

Underpinning the children's motivation for their learning experiences in the centres should also be their parents' and teachers' 'mainstream' expectations. The children knew that they had no choice but to commit themselves to the centres (see section 5.4.3). Therefore, they used any means that they could to mediate this process. Because the family cultural tools were familiar, applicable and useful, it is understandable that the children relied on them, especially in their initial attendance at the centres. The reasons that Leah (4:6/C) and Rick (4:5/AC) regularly played with Chinese peers and used family cultural tools in the centres could be: (i) their strong status in the Chinese peer groups established right from when they first attended the centres; (ii) them being sought after by newly-arriving Chinese peer; (iii) their longer time exposure to English; 
and (iv) their ease to apply the family cultural tools. Given that Leah was still concerned about her English ability (see section 6.3.1.1) and Rick was not absolutely competent with that language, it is possible that the two children preferred using the family cultural tools to those in the centres. It is evident that the children's choices about committing themselves to the centres agreed with the teachers and Chinese immigrant parents' expectations for the children's learning experiences, indicating that adult expectations did influence children's learning and development.

In saying this, what cannot be denied, is that although the parents expected the children to follow the centre's ways of doing things, because the parents were exercising Chinese cultural practices with children in their families, their family culture exerted strong contextual influences on the children in most of their learning experiences. Also, despite teachers' 'mainstream focus', many accepted the appearance of Chinese culture in their programmes and some even tried to include specific Chinese practices into the children's learning experiences. Thus Chinese cultural tools were prominent in both environments. Possibly because of this, the children felt free to resort to both sets of the cultural tools, and their actions became a site of cultural hybridity. The children were sorting out ways of relating the two cultures in order to learn and develop in their learning settings.

The children's use of the Chinese language with their Chinese peers and teachers, and the vital social bonds between them highlight the contributions of familiarities and similarities to a sense of belonging. According to Osterman (2000), a sense of belonging is the most essential need of human beings in their social worlds. Te Whāriki specifically articulates a sense of belonging as a learning goal for all young children, to be achieved through children "connect[ing] links with the family and wider world; [knowing] they have a place; [feeling] comfortable with the routines, customs, and regular events; [understanding] the limits and boundaries of acceptable behaviours" (Ministry of Education, 1996, p.54). In this research, the children's actions in many learning situations indicated that they had a need for a sense of belonging and as we repeatedly saw in Chapters 6 and 7, the children drew on specific cultural tools to achieve this. 
So, the process that the Chinese immigrant children were taking when learning and developing in New Zealand EC centres was one in which they applied specific cultural tools to generate their own repertoire of practice to achieve a sense of belonging. Given a range of cultures in their centres, the children chose to spend time with peers and teachers of similar cultural backgrounds. In the interviews with them, it became apparent that they chose to speak Chinese and to be with Chinese people because of their need for ease and clarity of communication. The children understood themselves as being Chinese and the usefulness of their language to help them make sense of the new learning setting. We have seen examples of the children using their family cultural mediators and tools to find out 'where to go', 'who to be with' and 'what to do', which provided evidence that the social groups formed between the study children and other Chinese people, provided them with an initial sense of belonging. The children's learning with Chinese peers and teachers laid foundations for them to move further into the centres' cultural community, a movement that Leah and Rick appeared to have made by the time that they participated in the research, which was about two years from their entry into the centre. Feng et al. (2004) have argued that the close group bond of children of the same language backgrounds enables them to use what they are familiar with, to deal with unfamiliar learning experiences.

My study indicated that the children's drive to belong in the centres was first facilitated through the use of their family cultural tools with Chinese peers and teachers and later through their use of the tools of both cultural communities. Through moving between the two cultures, the children created a new repertoire of practices and generated a useful intercultural way of being for themselves. As a consequence, the relationships between the two sets of cultural practices became a by-product of many aspects of the children's learning experiences.

More theoretically, findings of this research illustrate a way in which concepts of sociocultural theories, particularly languages, cultural beliefs and practices, cultural tools, guided participation, mediation and a sense of belonging can be positioned in intercultural contexts, where the children's family cultural practices, co-existed or cofunctioned with those of another cultural community. These findings potentially synthesise individual, sociocultural and intercultural aspects of development into a 
systematic illustration, which I call child-driven hybrid cultural mediation. This illustration is shown in figure 8.2.
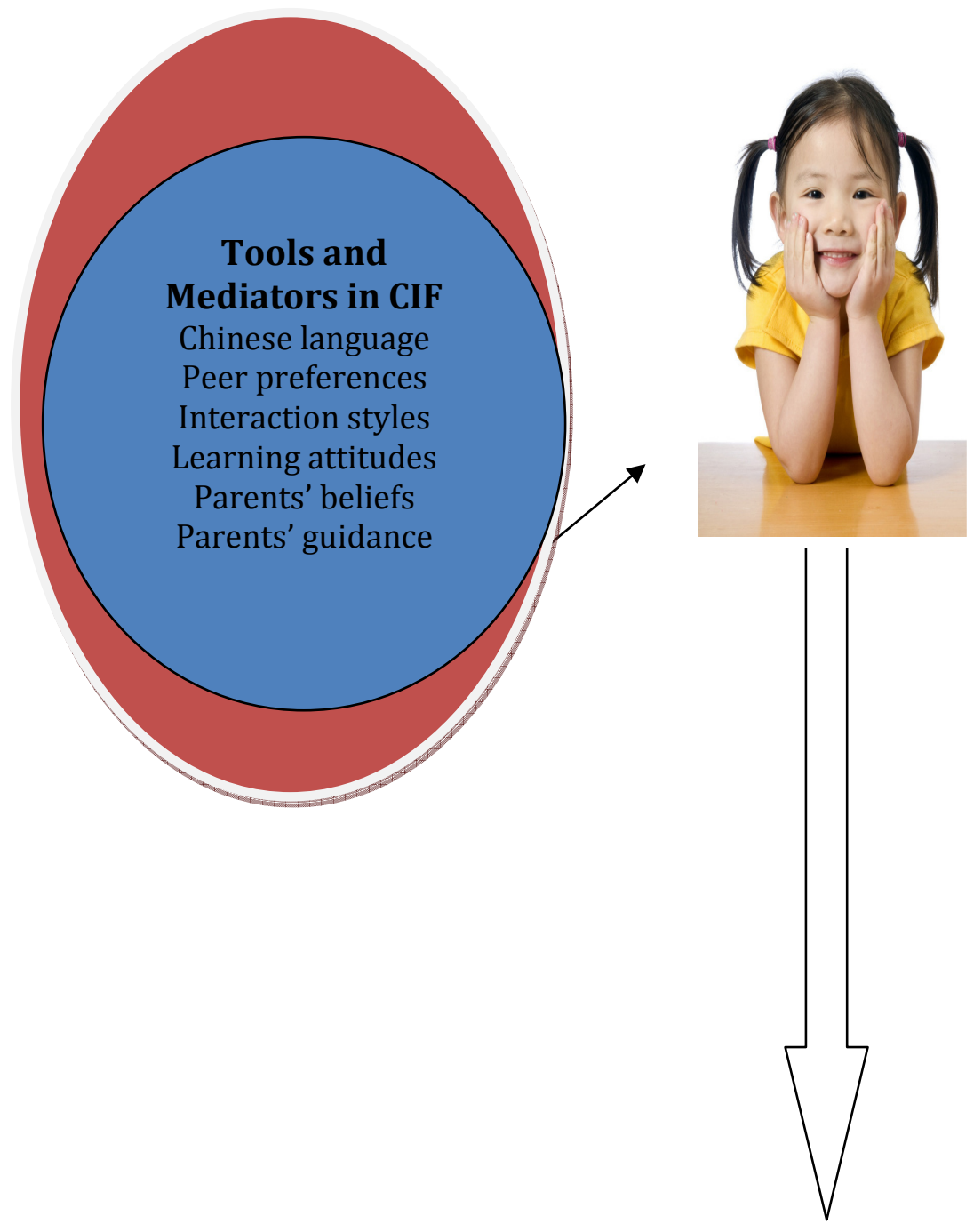

Generation of a new repertoire of practices

$\mathrm{CIF}=$ Chinese Immigrant Family EC= Early Childhood

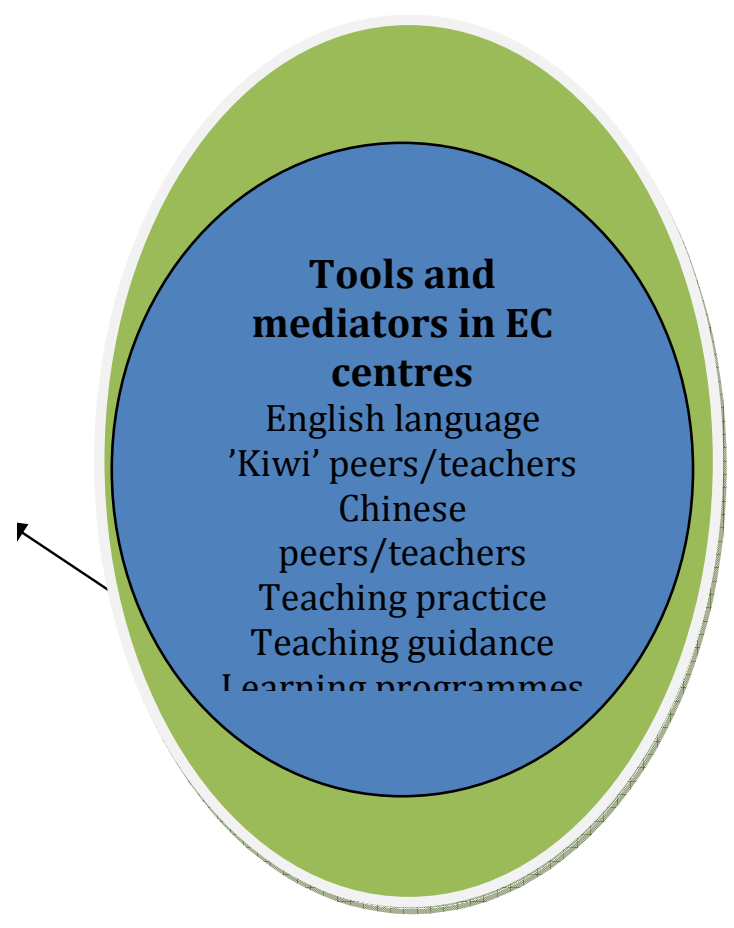

Xiaohan, Jim, Amy and Eden Use the tools of both cultural communities to:

- act in their learning settings

- frame their communicative exchanges,

- learn and use new cultural knowledge,

- engage in learning experiences with peers and teachers, and

- create inventive strategies.

\section{Leah and Rick}

Use the tools of both cultural communities to:

- connect links with the family

- find learning place with ease,

- fit comfortably with the routines,

- understand the limits and boundaries of acceptable behaviours 
The system depicted in Figure 8.2 unpacks the culture-culture relations within children's development. An intercultural research study like this, reveals the cofunctioning of different cultural tools and the effects of their dual existence on children's learning and development. In addition, the children's own motivation, their abilities to apply guidance, and the social guidance provided to them, were also important factors in their creation of a repertoire of practice, which led to their establishment of a sense of belonging.

These findings support a holistic view of learning for each child rather than a view of them being shaped by a particular culture. In Lantolf's (2004) reflection on sociocultural theory, he wrote:

Despite the label 'sociocultural' the theory is not a theory of the social or of the cultural aspects of human existence...It is, rather...a theory of mind...that recognizes the central role that social relations and culturally constructed artifacts play in organizing uniquely human forms of thinking. (p. 30-31)

Lantolf's quotation aptly captures the dynamics of learning experienced by the children in my research.

In this respect, the most secure overall answer to the key research question of "How do Chinese immigrant children experience learning and development in New Zealand EC centres?" appears to be that for them, the EC centre was an intercultural learning context where the children could maintain their family culture, and engage in family practices to learn those of another cultural community. In this context the children's intent was to achieve a sense of togetherness and belonging, both within the groups of their family culture, and the wider centre setting, using intercultural processes that can be characterised as child-driven hybrid cultural mediation.

\subsection{Looking forward: Educational implications}

The following section draws out educational implications of this research and provides discussion of the second research question:

How can Chinese immigrant children be supported towards a positive learning experience in New Zealand childcare settings? 


\subsubsection{Introducing a focus on Chinese language and Chinese immigrant family culture}

This study highlights the power of family culture to mediate the learning experiences of Chinese immigrant children in New Zealand EC centres. Implied in this finding is the importance of grounding the learning of a new culture in the utilization of children's family cultures (Brisk, 1998; Haworth et al., 2006a; Haworth et al., 2006b; Ryu, 2004).

In my research, Chinese immigrant family culture was reflected through a range of tools that the study children used in the centres, including the Chinese language, learning behaviours, attitudes towards teachers and social choices. It revealed that despite the various ways in which the children applied their family cultural tools in their centres, family culture played an important role in the development of new learning experiences for them all. Previous research has explained this phenomenon in a number of ways: family learning experiences provided children with enormous resources for learning (Igoa, 1995; Ovando, Collier \& Combs 2002; Ryu, 2004); family culture is represented by "essential cultural practices and bodies of knowledge and information" (Moll, 1992, p.21); and people who can speak the same language are able to relate and build personal bonds (Banks \& Banks, 2006). Given that the children in my study used their family cultural tools so naturally and consistently and these tools positively mediated many aspects of the children's learning experiences, it would seem important that learning programmes for these children should actively seek to include their family cultural tools.

The children's active drive of their own learning and development has important implications for ECE, especially the use of Chinese cultural tools in programmes. My study suggests that it is essential that children are provided with choices "to determine when to apply what approach" and "how to engage in an activity" (Rogoff et al., 2007, p.510).

The 'family-culture away phenomenon' in the cases of Luke and Sarah, highlights the paramount role played by Chinese peers and teachers in providing the study children with essential mediational tools from their families. This finding is consistent with previous research which has reported that when children and other people share common cultural backgrounds, "they are able to engage in productive interactions" 
(Callender, 1997, p.20); and the use of family language is "a means of access to new and complex concepts" (Igoa, 1995, p.38). It is reasonable for centres that have Chinese children and Chinese-speaking teachers to encourage them to interact with each other using their family cultural tools, particularly their language.

Regarding the EC centres where there was only one Chinese child attending, such as for Sarah and Luke, the family cultural tools that they could relate to for a sense of belonging and through which other children could gain an understanding, were significantly lacking. For example, when Luke spoke Chinese to his teachers, he would have expected a response that he could understand. Unfortunately, nobody in his centre could interpret his language, and thus his attempts to participate in his new learning setting were continually thwarted. This research points to a requirement for ECE programmes to incorporate cultural practices of the children's families. Given that no centre can guarantee the availability of Chinese teachers or children when a Chinese child arrives, it is important that all EC centres have access to the means to incorporate Chinese cultural practices, especially the routine use of the Chinese language, as an essential part of practice of all the teachers. This can be done, for example, by teachers attending workshops, asking Chinese-speaking parents to teach them, inviting Chinese speakers to contribute to the centre programme, or using language-learning technologies, such as videos. Although this research found that the children who had Chinese people in their centres had smoother experiences than those of Luke and Sarah, it is conceivable that if they had not been available, these children would have encountered similar difficulties to what happened to Luke and Sarah.

However, the major challenge to incorporating cultural tools of Chinese immigrant families is not EC teachers' lack of belief in the importance of these tools for supporting the learning and development of the study children. Instead, the challenge seems to lie in the high levels of belief among the teachers, Chinese and Pākehā alike, in the effectiveness of mainstream education. This belief appears to be so strong that although they also acknowledged the value of immigrant children's family resources, the teachers made few attempts to use them. The study found that except for using the Chinese language with the Chinese immigrant children and spending time with them when needed, Chinese-speaking EC teachers did not 'interrupt' the 'mainstream' programmes 
by initiating the practice of Chinese cultural tools, such as Chinese stories, or Chinese nursery rhymes.

The teachers' belief in the effectiveness of the mainstream education seemed to be pinned to their focus on child-centred pedagogies, which as I argued (see section 5.2.1) seemed to have reassured the teachers that their current practice was sufficient, thus effectively ruling out any effort to explore beyond the confines of the 'mainstream'.

These findings suggest three important implications. First, teachers should recognise the lack of neutrality of mainstream education. Second, teachers should at least be open to other cultural and pedagogical approaches, including tools of the cultures beyond those of mainstream New Zealand and thus extending sociocultural practice in EC centres. Third, teachers from minority cultures should initiate the implementation of their own cultural resources in the programmes.

Campbell and Page (2003) understood that mainstream EC teachers' upholding of mainstream education was created by a fear of challenge, because "what is understood as 'professional knowledge' and 'family or child knowledge'... [could] challenge and disrupt early childhood theory and practice" (p.295). While this research did not collect relevant evidence, it is possible that this fear could be present in some of my participants. Vedder, Horenczyk, Liebkind and Nickmans (2006) consider this and advise that "a strong attachment to one's heritage culture does not imply that the individual is not able to have skills in and a sense of belonging to additional cultures" (p.158). The message in this statement is therefore an assurance to teachers that it is acceptable to use family cultural tools for children of minority cultural groups.

It could also be that teachers did not have the knowledge about non-mainstream cultures, nor the necessary skills to incorporate tools of these cultures. In Chapter 5, there have been arguments over the teachers' generalised use of 'Chinese culture' to represent the culture of the study children and their families (see section 5.2.3). The examples in Chapter 6 illustrate Jim's and Luke's teachers' lack of response when the children talked to them in Chinese (see section 6.2.2.1). For these reasons, this research raises the implication that there needs to be a requirement for teachers in mainstream cultural 
communities to develop some knowledge and skills about the cultures of the children in their centres.

Chinese-speaking teachers maintained the beliefs and practices they had learned in New Zealand ECE. Child rearing and early education approaches that were applied in their families or the cultural communities were not used by these teachers in their work with Chinese immigrant children outside their common community groups. Their 'minority' cultural and ethnic identity might have prevented these teachers from actively using their 'minority' cultural resources and revealing their strengths. It seems important that teachers of minority cultural backgrounds are encouraged and supported to value, use, and extend their own cultural resources in New Zealand EC settings.

A further finding was that teachers implied that English was essential for the study children to settle into English-speaking environments, so there is a tension here between learning a new language and keeping the home language. Previous studies approve of teachers' efforts to help second language children with learning the second language because it is the dominant language (Siraj-Blatchford 2001; Tabors, 1998). The study supports this view too, after pointing out that one of the qualitative differences in the behaviours of the children who could use English well and children who could not was their ability to access many experiences of the centres.

However, in view of the positive contribution of the Chinese language to the children's learning experiences, placing too much focus on the development of English without actively seeking support from children's family language and culture would be regrettable for both teachers and children. Rhedding-Jones (2002) advises teachers: "We must be critical of the notion that the standard language must be understood before school is started" (p.100). Central to this view is that there is no need to narrowly and exclusively attend to the development of the dominant language for children in the EC period.

\subsubsection{Supporting the children's experiences with English-speaking peers}

It is clear from the study that the children were interested in playing with Englishspeaking peers. Evidence of children's desire for friendships has also been noted 
elsewhere (Howes, 1996; Johnson et al., 2000). However, as shown in Chapter 7, for some of the children, their attempts to interact with English-speaking peers resulted in undesirable behaviours, such as withdrawal or aggression. Sears (1998) argued "among second language children, making friends seems to mark a watershed in the process of adjustment" (p.102). Given the children's desire to interact with English-speaking peers, their lack of appropriate social competence, and the high possibility that Englishspeaking peers expanded their sense of belonging, it is important that Chinese immigrant children are supported to develop relationships with their English-speaking peers.

Supporting the study children's experiences with English-speaking peers is especially crucial when considering children in similar circumstances to Sarah and Luke, who did not have Chinese peers. A significant issue that the present study highlighted is the difficulty these children experienced in becoming engaged in peer relationships. They demonstrated some inappropriate social behaviours, in particular their diffidence and aggression. A close examination of the data reveals that a reason for these behaviours was probably that the children could not easily relate to peers. Teachers in this study did not appear to support these children to relate with peers but provided them with their own attention only. Given that the children's behaviours were primarily in response to their difficulties in working with peers, it is improbable that interactions with teachers could provide them with all the necessary social opportunities. While it is not absolutely necessary that the children should involve themselves in peer activities throughout the programmes, what happened to some children, especially to Luke and Sarah, calls for teachers' attention to children's peer relationships.

Two themes have permeated much of the research on supporting the peer experiences of immigrant children: the development of their social skills and the acceptance of them by English-speaking peers (Sears, 1998; Siraj-Blatchford, 2001; Tabors, 1998). As indicated in this study, the children's successful interactions with English-speaking peers occurred because the peers welcomed them and included them (see section 7.2.2). Teachers are urged to help immigrant children develop peer skills and encourage friendly attitudes and their acceptance by children of the mainstream cultural group. 
Previous research found that young children are aware of differences, develop a range of feelings about them and "in the active construction of their identities, children distance themselves from "others"” (Siraj-Blatchford, 2001, p.100). This study, too, has reported Luke's peers' rejection of his attempt to play with them, possibly because of their awareness of the difference between themselves and Luke (see the conversations between Luke's peers and his teacher Nicole in section 5.2.2). Because of this, it is important that teachers take the advice from Patary-Ching et al. (2006) to help Englishspeaking children understand the value of being different by showing them the strengths of individuals and providing them with opportunities to explore differences. Skattebol (2003) suggests that "children are encouraged to like difference" (p.162) and SirajBlatchford (p.101) says, "the early years curriculum should therefore incorporate work on children's awareness of similarities and differences, and help them to see this as 'normal"”.

My study of the Chinese immigrant children's peer experiences also found that the children showed a clear preference for playing with Chinese peers. For example, Eden's data indicated his exclusive interactions with his Chinese peers. Given the importance of children developing competences to function in the larger sociocultural community, what happened to Eden might need to call into question teachers' interventions, because Eden appeared to have missed out on the opportunities that he would possibly have gained from playing with non-Chinese speaking children.

There is a tension of ideas here. Although I support the view that Chinese children should have a wide range of peer experiences, evidence from the study, including the positive outcomes from interacting with Chinese peers and the difficulties when there were few, reminded me that teacher intervention into children's peer experiences should be approached with caution. In addition, based on his study that identified children refusing the peer groups chosen for them by their teachers, Keddie (2004) advises that children's peer preferences should underpin the teachers' support of children's peer experiences. When comparing children's opinions of their peers in China and New Zealand, Wang and Ritchie (2007) found that different cultures provide children with different ways of relating to peers. While it is important that children have many peer experiences, underlying all these findings is also the message that teachers' attempts to 
intervene in children's peer interactions, should build on children's own cultural and individual preferences.

Nevertheless, teachers should not lose sight of the significance of their roles in enriching children's social experiences. This is especially important for children like Eden who initiated no interaction with peers of non-Chinese cultural groups. Teachers' purposeful arrangement for him to play with English-speaking peers would have helped mediate his experiences on an intercultural path.

Johnson (1994) proposed two general principles to guide teachers' work in this area:

(i) create opportunities [for children] to interact in a variety of participation structures; and

(ii) build from children's cultural and individual orientations toward classroom interaction" (p. 185).

These two principles nicely tie together the central tasks that teachers can undertake to facilitate the peer experiences of Chinese immigrant children.

\subsubsection{Inviting parent participation}

While this research identified Chinese peers and teachers as the most direct mediating force for the Chinese immigrant children's use of family tools in the centres, it is conceivable that underpinning many actions of the children was the influence of their families. Experiences with parents in their families provide children with essential knowledge and appreciation of people, places and things (Carr, 2001). In this research, apart from the Chinese language and social preferences, the children's persistent and committed learning behaviours and their attitude to teachers, were also important aspects of their family cultural mediation.

The lack of parent input into the children's centre programmes was a clear phenomenon across all cases in this research. This finding is consistent with other studies that have investigated Chinese parenting practices (Dyson, 2001; Guo, 2006). Clearly this phenomenon is not ideal, given the research evidence that limited involvement of Chinese immigrant parents in their children's EC centres brought two issues to the foreground: (i) the learning experiences of the children in the centres were not informed 
by their family experiences; and (ii) teachers and parents did not work together in a learning community.

In a study which investigated Chinese immigrant parents' parental styles, Dyson (2001) reported that Chinese immigrant parents' direct 'training' of children could have explained their lack of participation in schools. My research likewise indicates that the Chinese immigrant parents preferred to train their children in their homes rather than involve themselves in the EC centres' programmes. Furthermore, the parents' expectation of mainstream education for their children as well as their perception of the insignificance of their involvement in their children's centres, also led them to want their children to follow what they termed 'the Kiwi culture'.

However, what this research found is that the non-participatory behaviour of Chinese immigrant parents is not purely a cultural or personally-valued practice, but has implications for the relationships between the parents and teachers. Limited engagement of Pākehā teachers with Chinese immigrant parents could have contributed to the parents' hesitation about informing teachers about what they required. The absence of attempts by the Chinese-speaking teachers to help Chinese parents address their needs in the EC settings led the parents to think that teachers were not very helpful (see section 5.3.3). As a result, the parents made a tacit decision to comply with the centres' ways. Therefore, many important aspects of the children's family learning experiences were not incorporated by the teachers, who understandably then practised in their ways. Thus, learning for the children in their EC centres became increasingly an experience that was inconsistent with their learning processes at home.

Lack of interactions between the Chinese parents and most teachers contributed to some unfortunate experiences for the children, such as their perception that parents were only passers-by in the centres. In addition, Sarah and Luke's difficulties in developing a sense of belonging in their centres, due to the lack of Chinese support, illustrate a need for the centres to elicit the involvement of Chinese parents to help teachers work with these children. Klein and Chen (2001) provided a similar suggestion, stating that parent participation in children's learning programmes was crucial in helping immigrant children succeed in their learning experiences in out-of-home learning settings. 
Analysis of the data demonstrates that underpinning Chinese immigrant parents' minimal participation in the EC centres is also their lack of confidence in relating to teachers as well as the teachers' uncertainty about working with these parents. Addressing the same issue, Ovando et al. (2002) stated that the limited interactions between the mainstream teachers and language-minority parents could reflect their unfamiliarity with each other:

... many language-minority parents are very uncertain of their role within the school, and they may have a fear of being judged negatively by school personnel because of their lack of education or their limited familiarity with the dominant cultural systems. It is natural for people to feel ill at ease in unfamiliar territory, and schools often feel extremely unfamiliar to language-minority parents. (p. 426)

In the above account, it is clear that it is important for parents and teachers to be familiar with the roles and cultural practices of the other party. Rogoff (2003) uses the term 'cultural practice' to highlight the importance of cultural ways of doing things in daily learning activities. The concept of 'learning communities' too, emphasises linking people together for learning. The implication that could be drawn out of this study, in recognition of the significance of culture for learning and the concept of 'learning communities', is that teachers should include parents in such a way that they establish strong and consistent contacts (Brisk, 1998; Robinson \& Jones-Díaz, 2006). Furthermore, Vedder et al. (2006) point out that beyond contact, "it is important that groups enter the contact situation with equal status... and the contact must occur in the context of supportive norms" (p.159). Based on this study, a valuable support that Pākehā teachers and Chinese immigrant parents could use is Chinese-speaking teachers. When encouraged and provided with opportunities to act for both cultural communities, Chinese-speaking teachers should be able to act as a link between the cultures.

\subsubsection{Embracing diversity within multicultural contexts}

Working on cultural diversity was not identified as a strong experience for the adult participants in this research. As mentioned above, this may in part be due to their takenfor-granted expectation of a 'Kiwi' way of educating children in the centres. All the adult participants, teachers and parents alike, appeared confident about their beliefs and practices regarding mainstream education as a useful mediator for the study children's' learning and development. The incentive for the parents with this mainstream goal is for 
their children to blend into mainstream culture, thus functioning confidently in another cultural society (Gorman, 1998; Li, 2001). From the teachers' perspective, because they saw Chinese immigrant children as only needing 'more teacher attention', the learning programmes they provided for other children were expected to work similarly for the Chinese children.

As noted in Chapter 5, the parents in this study never asked teachers to provide their children with a Chinese style of education. Their acceptance, or indeed embracing of mainstream education, together with teachers' comfort with, and confidence in, the effectiveness of mainstream education could have limited teachers' attempts to work with other cultural practices. As a result, teachers could not easily sense the importance of addressing differences.

Robinson and Jones-Díaz (2006) suggest that it is problematic to avoid differences because being different is a reality, and children are aware of people's differences from very early ages. The behaviours of many children in this study confirm this. Skattebol (2003) says, "Human difference must be named to be discussed" (p.155). These ideas are calling for a move away from avoiding differences.

A focus on embracing diversity has implications for transforming teaching practice in a multicultural context that emphasises critiquing existing power relationships between the dominant culture and other cultures, and connecting cultural groups as equals (May, 1999; Vedder et al., 2006). Rhedding-Jones (2002) advises teachers to see their "own ethnicities as nationally, linguistically and religiously related" (p.92). Revolving around this is the adoption of "a reflexive approach to their practice with children and families" (Robinson \& Jones-Díaz, 2006, p.169), which would help teachers raise self awareness of subjective positions and form the baseline for their work with other cultures.

My study suggests that being uninformed of each other's cultural practices also emerged as a theme which explained teachers' and parents' hesitation to address differences. Regarding this, Vedder et al. (2006) claim that the key to succeeding is to support the minority people to value their cultural practices, and develop confidence to use them with people of the mainstream cultural groups. As noted in Chapter 5, the parents in this study did not perceive the inclusion of Chinese cultural practices in their children's 
centres to be of particular importance, a perception that contributed to their adoption of mainstream educational goals for their children. If the parents had been empowered to value the importance of including their family cultural tools in the centres for making significant and effective changes to children's learning, I expect that they would have participated and possibly shared with teachers their family-cultural beliefs and practices. Thesing (2008) advises that there is a need to support parents' sense of wellbeing and confidence in their role. Zwanns, van der Veen, Volman and ten Dam (2008) point out the necessity of having teachers experience tense situations in intercultural education so that they can identify them, evaluate their significance, and choose appropriate reactions to them. This will equip teachers with knowledge about intercultural issues, thereby enabling them to react to the tensions that emerge from cultural differences.

\subsubsection{Developing teachers' professional strategies and relevant educational policies}

Glynn (2003) reviewed existing educational policy and practice for New Zealand immigrant learners and identified two crucial areas for further work. They are "develop[ing] more responsive and inclusive learning and teaching strategies... and a more focused and integrated policy development" (p.273). Similar implications arise from the present study.

Most teachers in this study were experienced and qualified EC Pākehā practitioners and displayed many teaching competencies. Even so, the areas that emerged in this research for their professional development call for important attention to be paid to the teachers' perceptions and responses to the changing and complex cultures in their work settings. Ample evidence from the study indicates that "teachers' work with families, particularly with diverse families in a multicultural environment, [is] 'complex and challenging...Although teachers are entitled to give guidance and support to parents from their general training...their work ... [is] more complicated" (Chan, 2004, p.261).

Hughes and MacNaughton (2002), as well as May $(1999 ; 2004)$ argue that developing knowledge and skills for working with immigrant children rests on teachers' continuous deconstruction of their practice, implemented by critically challenging the status quo and their longstanding pedagogical beliefs and practices. At the heart of this view is the transformation of taken for granted ways of teaching and to "locate minorities as 
resources" (Rhedding-Jones, 2002, p.111). In doing so, it is essential that teachers engage in in-depth exploration of the strengths of the children and the families they work with (Hughes \& MacNaughton).

It has been widely recognised that pre-service and in-service teacher education are still the major vehicles for teachers' professional development (Robinson \& Jones-Díaz, 2006). This, in Gay's (2000) words, means that "knowledge plus practice is imperative" (p.191). In their advice to pre-service providers, Nilles, Alvarez and Rios (2006) recognise the integration of knowledge and field experiences, claiming that "without a coherent, coordinated and robust teacher preparation programme and accompanying field experiences, we cannot expect teachers to be qualified nor successful with SLL [second language learners]" (p.43). It is important that the professional development agencies provide opportunities for diverse cultural understandings and equip teachers or student teachers with the necessary knowledge and skills to create a multicultural learning environment. In recognition of the possibility of teachers' participation in inservice professional development being only voluntary, Gay states that these opportunities must be made mandatory. For the purpose of increasing EC teachers' multicultural knowledge and practices, New Zealand Teachers Council (2009) drafted Registered Teacher Criteria. One of the overarching statements of this draft states that "In an increasingly multicultural Aotearoa-New Zealand, teachers need to be aware of and respect the languages, heritage and cultures of all ākonga [learners]". The Teachers Council expects that the Registered Teaching Criteria will be made mandatory in 2011. This requirement for teachers is potentially very enlightening, because it conveys a message to teachers regarding the importance of multicultural practices in their teaching.

This study regards teachers as the key agents for improving the study children's experiences, However, given the complexities of the issues involved in working with these children, I agree with Rhedding-Jones (2002) that teachers need assistance from other people, particularly the policy makers because "professional learning appears to be considered by policymakers" (Hedges, 2007, p.274).

This study indicates that what should be attended to first in policies is the provision of time for teachers to relate with parents. The teachers appeared to have time for interactions with individual children during the sessions, but time was seen as a barrier 
for them to interact with parents at arrival and departure times. Given that the construction of partnerships with parents should mainly occur through regular face-toface communication (Hughes, 2003), certain lengths of time should be provided for direct interactions with parents, especially with those like Leah's mother who was willing to stay in the centre for a period of time.

Home visiting is a desirable practice suggested by a number of researchers (Brisk, 1998; Hetherich, Hughes, Page \& Campbell, 2003). Brisk justifies this by saying that “knowing their students' families changed teachers' attitudes" (p.82). Vedder et al. (2006) point out the inadequacies of educational institutions as major arenas for contacts, because they place constraints on outside people to feel empowered and acknowledged. Remembering the parents' reasons given for their minimal involvement in the centres, such as lack of time, and lack of confidence in approaching teachers, home visits could be a useful approach for teachers.

The value of bilingual teachers was explicitly identified in this study, which points to a need to utilise them to support children's experiences because: "they play a crucial role in observing, planning and implementing experiences collaboratively with other staff members in which their language expertise is valued and integrated across curriculum areas" (Robinson \& Jones-Díaz, 2006, p.124). Employing teachers of minority cultural backgrounds, therefore, is an important consideration. Moreover, with regards to the limited efforts by the Chinese-speaking teachers in this study to create culturally diverse EC communities, it is clear that employing teachers of minority cultural backgrounds is not the sole priority. These teachers need to be supported to advocate for their own cultures, for example, to facilitate professional learning opportunities for their Pākehā colleagues to learn about other cultures.

Another implication of this study for policymakers is the position of languages in the curriculum. Because of the value of home languages to mediate learning and development of young second language learners, language experiences should be provided both in English and children's home languages.

The research identified no multicultural policies in any centres. One explanation could be that, whilst there is a strong emphasis on the integration of minority cultural aspects, 
multicultural practice in a widely inclusive way is not explicitly suggested in $T e$ Whāriki. For Rhedding-Jones, Te Whāriki, as a bicultural curriculum statement considers minority culture to be mainly that of Māori:

There is no mention... of the children ... whose families migrated from Asia, Africa, or Eastern Europe...Constructing a double difference and a double resource, as in bilingualism, is thus done well in New Zealand. But moving toward fluid multiplicities, where people belong to a range of simultaneously operating categories, beyond a binary, remains a challenge. (Rhedding-Jones, 2002, p. 94)

Moreover, Macartney (2008) believes Te Whāriki and other supporting documents make no attempt to critique normalizing discourses. Thus, when the teachers in the present study embraced these documents to guide their practice, it is understandable that they would not be likely to examine it at a deep level.

These points give rise to the need for reviews of relevant policies so that the needs and expectations of Chinese immigrant children and parents in particular, as well as those from other minority cultures could be appropriately met.

\subsection{Chapter summary}

In summary, the learning experiences of the Chinese immigrant children in New Zealand EC centres were:

(i) mediated by the application of cultural tools of Chinese immigrant families in the new learning settings;

(ii) facilitated by the associations between the Chinese immigrant family culture and the culture of New Zealand EC centres;

(iii) impeded when Chinese immigrant family culture was unavailable in the centres; and

(iv) driven by the children's own agendas to achieve a sense of belonging and to develop appropriate repertoires of practice within their new cultural setting.

In other words, the study children's learning experiences in New Zealand EC centres could be described as occurring within a process in which the children associated their family culture with the mainstream culture of New Zealand EC centres against the 
backdrop of the wider sociocultural context. When the children could find their family culture and apply its tools alongside, or together with the practices of another cultural community, they found appropriate means to mediate their learning experiences. When their family culture had to be kept away, the children faced difficulties.

However, despite the value of Chinese immigrant family culture to positively mediate the children's learning in non-Chinese cultural communities, the experience of one child, Eden, cautions against a definitive conclusion that family cultures provided appropriate contributions to development in another cultural learning context for every child. Based on Eden's experiences, which showed his persistent attempts to keep himself aside from the mainstream culture of the centres through his constant application of the family cultural tools with his Chinese peers, the research indicates that the extent to which those tools are included in the children's learning experiences needs to be considered. Many examples in this study highlight that the significance of family cultural tools for children's learning and development of a new culture mainly lies in the close associations of these tools with those in the new cultural community. This brings to the fore the importance of establishing an intercultural environment for immigrant children. While it is understandable that the study children relied mainly on their family culture when new to the centre, they should be supported to move on to an intercultural path that enables them to competently cross the cultural boundaries.

From a conceptual perspective, the research indicates that understanding of Chinese immigrant children's learning experiences can be effectively achieved through consideration of the co-existence of the two different cultures in the wider sociocultural learning context of New Zealand EC centres. Many specific tools that belong to each culture can be unpacked as influencing the children in their own ways, alongside or together with those of another cultural community. Each child's own contribution to the learning process emerged from the need of the child to build a sense of belonging in the new learning setting, constructing experientially unique and hybrid cultural ways of learning and development.

The next chapter draws together conclusions, specifies the contributions and limitations of this research, and suggests areas for possible further research. 


\section{Chapter 9: Conclusions}

\subsection{Introduction}

The purpose of this study was to investigate the learning experiences of Chinese immigrant children in New Zealand EC centres. Using sociocultural perspectives, learning and development were defined as an evolving process embedded in socially and culturally mediated experiences in communities of practice (Rogoff, 1998; 2003; Vygotsky, 1978; Wenger, 1998). Findings of this research were thematically analyzed and interpreted according to a range of sociocultural concepts and perspectives.

Firstly, the research was premised on Vygotsky's model of sociocultural mediation of learning and development, with particular attention paid to languages and peer relationships as cultural tools. Like other researchers working within sociocultural frameworks (e.g., Foster \& Ohta, 2005; Haworth et al., 2006a; Haworth et al., 2006b), I worked to illuminate how language and peer relationships mediated the learning experiences of the study children in English-speaking EC centres.

The adoption of Rogoff's $(2003 ; 2008)$ theoretical idea of transformation through guided participation necessitated investigation into the processes of the children's participation in their cultural communities through engaging with others in explicit or tacit collaborative relationships. The children's own construction of hybrid cultural practices through coordinating between what they knew and what they were learning to know was also discussed.

Leont' ev (1981) and Engeström (1987) locate their arguments about learning and development particularly at the level of activity systems, namely one's learning contexts. Drawing on their concepts of 'activity theory', 'activity system' and 'expansive learning', this research recognised the children's learning as a distributed process and their learning contexts as (i) a 'web', a functional system in which the children, other people and their cultural tools were woven together; and (ii) 'boundary zones', that were connected through cooperating with boundary objects towards common goals. 
The concepts of togetherness, sense of belonging, peer culture and learning community informed my investigation of the joint involvement of the children and other people in cultural activities, to identify how these experiences influenced the children's evolving participation in their new cultural communities. The perspectives of multiculturalism and cultural diversity provided a lens through which I positioned the understanding of Chinese immigrant children's immediate experiences in a wider sociocultural context, that consisted of both their families and the mainstream culture of the centres. In doing so, I was able to discuss how Chinese immigrant children and their families experienced their lives in a different cultural community and how the cultural relations influenced their experiences.

The eight study children came from six EC centres. Sources of data included child interviews and observations, and parent and teacher interviews. The research design, processes of data analysis, and reflection on the research process are presented in Chapter 4. The choice of research methods and data analysis reflected the phenomenological and sociocultural perspectives considering personal accounts and social factors as critical for understanding lived experiences. The data revealed the richness of the children's experiences in New Zealand EC centres. Chapters 5, 6, 7 and 8 discussed the children's experiences using sociocultural contexts, languages, peer relationships, cultural relations and children's own drive for learning and development as the access points.

This chapter is a summary of the key findings and a discussion of the contributions of the research to current understandings about sociocultural theory and knowledge. It concludes with a discussion of the limitations of the research and some suggestions for further research.

\subsection{Key findings}

The key findings in relation to the first research question How do Chinese immigrant children experience learning and development in New Zealand childcare centres? are that the children's experiences reflected the intercultural relationships between the Chinese immigrant family culture and the mainstream culture of the New Zealand EC centres they attended. Most of the study children used their family culture as a base for 
their learning and development in the EC centres. Positive learning experiences occurred when the two sets of cultural tools were brought together, or when the children found opportunities to maintain their family culture in their own way, alongside that of New Zealand EC centres. The children faced difficulties settling in their centres when they could not locate their family culture there. In general, this research highlights a range of possible intercultural relationships that to date have not been adequately studied in EC settings in New Zealand. In particular, there have been no New Zealand ECE studies that have explored immigrant children's learning and development that comprise two or more sets of cultural tools.

The findings in relation to the second research question How can Chinese immigrant children be supported towards a positive learning experience in New Zealand childcare settings? are that these children should be offered opportunities to relate family cultural tools to those of New Zealand EC centres, and also to maintain the integrity of their family culture, for example, by having the opportunity of only speaking Chinese with Chinese-speaking people. Overall, this research suggests that educational support for Chinese immigrant children must include strategies that support them being proactive in developing a repertoire of practice that supports their engagement in intercultural learning settings.

\subsection{Contribution to theory and knowledge}

This research is the first in-depth case study investigation into the experiences of Chinese immigrant children in English-speaking EC centres in New Zealand. It yields the following significant contributions.

The theoretical significance of this study is that it has confirmed the power of family culture and family learning experiences in mediating the learning experiences of children in another cultural community. Chinese immigrant family culture was a substantial and important learning support for the children who were able to access it.

My research demonstrated that Chinese immigrant children were committed learners and strategic constructors of their own learning experiences. This finding is consistent with previous research reporting that Chinese children are keen on learning (Li, 2004; 
Li \& Wang, 2004), but adds the aspect of the children's active drive for their own learning and development. This finding draws attention to the strategic and adaptive attributes of the children, including not only their efforts to embrace and succeed in the new learning context but also their endeavours to support their own learning and development pathways.

Being part of a group and having a sense of belonging are confirmed as the children's most crucial need in their centres, and it became the basis of their general learning experiences. A conclusion therefore is that whilst learning is an externally mediated process, it is very much driven by children's own motivations and desires.

Examples of the Chinese immigrant parents' 'mainstream goals' demonstrate the transformation of their beliefs and practices, which are influenced by their experience as an ethnic minority in a new sociocultural context. Parents' perception of the importance of mainstream education and their lack of recognition of the significance of family culture for children's learning in outside environments, resulted in their keeping the family culture at home. In relation to this finding, my study found that the concepts of diversity and multicultural education were implemented in a superficial manner in the practice of the New Zealand EC centres in this study. From the teachers' perspectives, the limited incorporation of relevant cultural tools seemed to arise from their strong belief in the effectiveness of the mainstream education, and from the limitations in the teachers' repertoire of teaching practices. It was argued that the teachers' conclusions about the same pedagogy being appropriate for all children did not support the aspirations of honouring diversity present in educational documents that are meant to guide early childhood services; this was also the case with regards to the principles of multicultural education. The research, thus, has implications for the theory and practice of cultural diversity and multiculturalism, because it indicates the need for further examination of the issues of equality, transformation, the practical and theoretical positioning of culture, and the relative positioning of the mainstream culture and other cultures in New Zealand EC services.

This study makes contributions to the sociocultural theories presented by Rogoff (2003), Vygotsky (1978) and Wenger (1998) by providing an exploratory application of how they relate to learning and development of one cultural group of children in the 
particular setting of New Zealand EC centres. Informed by sociocultural concepts, this intercultural research provides a specific lens to identify the complex relationships between language and peer experiences, first language and second language, own culture and other cultures, in relation to similarity, difference and transformation. In the current study, these elements intersected with each other to mediate the experiences of learners in a holistic manner.

While this research is congruent with Vygotsky's view that learning and development is a mediated experience, his view has been extended in this study to an exploratory application in the context of intercultural learning settings in a New Zealand context. Although Vygotsky accessed the experiences of a range of ethnic communities in the Soviet Union, I have not located information that he investigated intercultural data as were obtained in this study. My intercultural data has contributed to a further understanding of mediation in sociocultural context where two different sets of cultural tools respectively or mutually exert their mediational forces, visibly or otherwise.

The intercultural possibilities identified in this research also reinforce the theoretical notions of 'activity system' and 'boundary crossing' (Engeström, 1987; Leont' ev, 1981). Within the framework of intercultural possibilities, the boundary zones appeared to be complicated activity systems of the Chinese family culture and those of the EC centres. The 'boundary crossing', in this research, was revealed in the practical ways in which the two cultural communities were related.

This research also highlighted children's individual abilities to actively develop and employ their family cultural tools to mediate their own learning and development. These tools became such because of their use by the children. If the children in this research had not actively applied the cultural tools, the tools would not have functioned as tools. For example, the tools of Chinese immigrant families did not function for the two Chinese children who did not use them in their centres where they were the only Chinese language speakers. An intercultural research study such as this has the advantage of being able to overtly capture children's abilities and learning strategies, possibly because the process of developing intercultural competencies required the children to take more strategic actions (Carlson \& Meltzoff, 2008). 
The finding of child-created hybrid cultural mediation is an extension of Rogoff's (Rogoff et al., 2007) concept of hybrid forms of practice. Within Rogoff's perspectives, the development of hybrid forms of practice is the result of an adjustment or redevelopment of children's original repertoire of practices. It is evident in this research that the children did not simply adjust or re-develop their own cultural systems when trying to construct hybrid ways of learning. Their family cultural tools were employed in interactive and converging ways with those of the new learning environment.

The study also adds to Rogoff's (2003) thinking on collaboration that emphasises shared engagement between people. The findings indicate that Chinese immigrant parents' embracing of 'Kiwi' culture, some teachers' incorporation of Chinese culture, and the children's integration of both cultures are collaborations that may not always be explicit. In some settings, particularly in intercultural encounters, collaboration among people and between cultures could be a natural outcome that remains implicit in practice and not specifically articulated.

\subsection{Research limitations}

My study has investigated the learning experiences of eight children from six EC centres in a major New Zealand city, which could be considered to be a small study. However, sociocultural and phenomenological research aims to obtain an in-depth picture of the richness and complexity of experience and this was the aim of the current research. Perhaps the small number of children in this study and the limited range of centres means that further research is required, preferably with larger groups of participants before wider generalizations for practice can be interpreted.

The intention was to include both parents as participants in the study, however, mothers were the predominant participants. This may have produced limited perspectives from parents, given that mothers and fathers may have different perspectives on children's EC experiences. Furthermore, the parents in my study were predominantly educated and voluntary immigrants, who were seeking to enhance their life opportunities. These findings may not apply to families who migrated for other reasons, such as family reunification or on refugee status, because individuals who migrate for different reasons 
are likely to have different ways of parenting their children (Pachter \& Dumont-Mathieu, 2004).

The centres in this study were located in geographic areas with a high concentration of Chinese residents. This may mean that Chinese cultural practices among the children were already well established there, as illustrated by Leah and Jim's teachers' comments that it was a typical phenomenon in their centre that Chinese children played with each other because of the regular intake of Chinese children. Thus, a new Chinese child could easily locate themselves into existing Chinese peer groups. Therefore, it is possible that different dynamics may emerge if similar research were carried out in centres isolated from the Chinese community.

The centres were selected because the teachers wanted to enhance their professional skills and to increase their understanding to support Chinese immigrant children. While the teachers' interest and willingness to participate in the research were of great benefit to my study, the findings may only reflect the practice of teachers who had strong intentions to support Chinese immigrant children. These teachers could be more likely to be trying out appropriate ways of working with Chinese immigrant children (e.g. using speaking techniques, attending learning sessions, incorporating certain types of Chinese culture) than those who did not participate in this research. Perhaps the findings generated from them are more positive than would have been the case from other teachers.

The timing of the data collection provided access only to the experiences of the children at that point of their childcare attendance. If the timing of the data collection had been different, different experiences of the children might have been forthcoming which may in turn have produced different interpretations and explanations. Moreover, if the children were observed over a longer time period, their trajectories over time in adjusting to their EC centres could have been noted.

In addition, my role as a Chinese immigrant parent and an EC professional, as discussed in Chapter 4, undoubtedly influenced some of the findings, because teachers, parents and even children might have responded and behaved differently with a person they could not so easily relate to. On the other hand, I also consider my Chinese identity to 
have been a strength throughout the research process, at least in interviewing the parents. The parents might not have provided so many opinions if the study had been conducted by a non-Chinese person, or a person who could not speak Chinese with them.

Another limitation of this research, in relation to my own Chinese identity, and my experience of mothering two children in New Zealand, is that the findings and conclusions of the research could have been biased by my own expectations and practices. While many efforts have been made to strengthen the validity and quality of the data analysis, a further peer-analysis throughout the project would have provided a valuable cross-check and extension.

On reflection at the end of the project, the inclusion of Chinese-speaking teachers along the same lines as their English-speaking colleagues appears to have been an inconsiderate decision of the research as it has resulted in very limited data about the practical work of these teachers with the Chinese immigrant children and their families. Specific attention to Chinese-speaking teachers' insider knowledge would have helped this research gain more insights.

\subsection{Future research}

The main focus of this research is the learning experiences of Chinese immigrant children in EC centres. I chose to use EC centres as the only context of child observations, but it would be useful for further research to also observe Chinese immigrant children's learning and development in their homes. This would generate additional insights into the children's experiences as they learned to function in their centres whilst also being a member of their family culture.

Rogoff (2003) has conceptualised learning and development as a transformation of cultural participation. A longer and more intensive exploration of children's learning experiences could document how their participation shifts and changes.

This research identified strong friendship patterns among the Chinese immigrant children, which could be partly attributed to their being over three years of age, Thus they knew more about their family culture and were more able to seek culturally-related 
relationships than toddlers and infants. A study on children of younger ages is necessary as well, as Chan (2006) reports "the two Chinese toddler participants, who could speak Chinese fluently, hardly said anything in the centre, neither English nor Chinese. They did not even communicate with each other in their first language" (p. 36).

Peer relationships were only one aspect of the focus of this research. Research in the area of immigrant children has indicated the need to look at peer experiences in their development (Deegan, 1996; Tabors, 1998). This research found that peer experiences dramatically influenced the children's learning and development. A further study focusing on that is therefore necessary. Similarly, the attitudes and perceptions of children of mainstream cultures to immigrant children could provide further insights into how to facilitate positive peer experiences of immigrant children.

During this research, I became more aware of the partnership practices of the teachers and Chinese immigrant parents, particularly their absence. An investigation of teacherparent partnerships from a cross-cultural perspective could be very informative.

Providing children with mainstream education in New Zealand EC services was the key and highly valued goal for both the Chinese immigrant parents and New Zealand EC teachers, and exploring this phenomenon more widely could be useful. It would be significant for a study to be conducted with people from mainstream and minority cultural communities to identify the factors contributing to their cultural preferences in different contexts.

The competencies demonstrated by the children and parents are worth further exploration. While this research documents certain competencies such as the children's commitment to learning and parents' determination to help children settle, it is important to explore these further, given Rhedding-Jones' (2002) statement that "the 'competency' developed in minority households appears to be neither named nor recorded" (p. 91).

In this research, language and peer experiences were related in both positive and negative ways. It seems important that a study pinpoints these aspects for immigrant children to see to what extent language and peer skills influence each other. Action 
research that elicits actions for change might be an appropriate methodology for investigating this phenomenon.

Although this study suggests that first language Chinese-speaking teachers play an important role in the learning experiences of the children, only three Chinese-speaking teachers participated in the study. They were not singled out for their special cultural backgrounds but included in the study as teachers in the selected EC centres. Upon completion of the data analysis, it became obvious that further investigations into Chinese-speaking teachers' own experiences and perceptions of working with children of the same cultural groups in a different cultural setting would yield useful information.

Potentially, a study including Chinese fathers could create different but equally useful information because in Chinese culture, fathers and mothers differ in their parental styles (Lau, Lew, Hau, Cheung \& Berndt, 1990). Moreover, according to Chan and Leong (1994), Chinese males and females respond in different ways to changes in their roles and status in immigrant countries. If that is the case, it is likely that Chinese immigrant fathers have differing expectations and practices from Chinese immigrant mothers.

As a result of my field work experiences with the Chinese immigrant children and their parents, especially with Eden's mother, this research raises a need to understand, and to resolve the tension between Chinese immigrant children's rights for protection and their parents' traditionally upheld rights and responsibilities to use their own ways to protect their children from learning inappropriately (see section 4.8).

Another possible line of research enquiry that can be drawn out of my field experience in the current project would be a focused investigation into research ethics with Chinese immigrant parents/populations. It seems important that ways of maintaining ethical standards are identified that could be used in research with Chinese communities.

\subsection{Final remarks}

The experiences of the Chinese immigrant children in New Zealand EC centres can be summarised as a process of negotiating and reconciling the new and old, a response to 
uncertainties and familiarity and a demonstration of individual motivation and cultural reliance. Similarities and variations in their learning experiences were identified.

This research highlighted the EC centre as a useful context in which to understand the intercultural experiences of immigrant children, immigrant parents and New Zealand EC teachers. In Rhedding-Jones' words, EC centres can be located "as a critical site for cultural encounters" (2002, p.90) and for Thesing, they are "a community network" (2008, p.66). In this research, the EC centres framed the research context and indicated the children's wellbeing, sense of belonging, communication, contribution and exploration in practice (Ministry of Education, 1996). They also acted as a lens through which people's positions in their own culture and other cultures were viewed.

Many of the findings of this research confirmed or strengthened what we already know about Chinese children, Chinese culture and the experiences of second language speaking children. These findings also suggest a new area of enquiry in terms of the interplay and co-existence of two cultures in intercultural research, and the relationships of these cultures on Chinese immigrant children's learning and development.

The teaching experiences identified in this research are complex, involving questions of the personal, intercultural, sociocultural, practical and political positioning of a wide range of issues in relation to teachers' work with diverse cultural children and families. From a sociocultural perspective, the implications that the study has drawn for EC teachers are profound since a shift in their beliefs and practices is required for them to respond visibly and appropriately to diverse cultures. This line of thought also leads to the suggestion that teachers need ongoing professional development and support to develop and implement appropriate policies and practices.

The research provided evidence of many Chinese immigrant children's successful adaptation to their learning programmes. This success can be attributed to:

(i) the children's and parents' high motivation for their children to adjust in the centres;

(ii) children acting as active drivers of their own learning;

(iii) children's persistent use of the Chinese language;

(iv) the availability of Chinese speaking teachers and peers in some centres; and 
(v) the focused attention that the children received from their teachers.

While the children were exposed to two differing cultural concepts and practices, their search for linkages facilitated their transition to a new cultural setting. Therefore, it is essential that educational programmes facilitate the linkages through strategies that enable relationships between different cultures, so that immigrant children's learning and development in the new and first cultures are balanced. In doing so, it is important to include the children's own drive, their parents' motivation and cultural information, the knowledge and expertise of bicultural teachers, and 'Kiwi' teachers' strategies of working with children.

My role in the research process was also a source for reflection. Throughout this journey, I was constantly trying to reconcile my role with the role of participants, and my interpretation of the phenomenon with the views of the participants. The view that the relationship between the researcher and participants is the key to sociocultural and phenomenological research contributed to my endeavour to establish findings that illustrate the participants' own perceptions of their experiences.

My aim for this study has been to provoke thinking on how to support Chinese immigrant children so they, in the words of Siraj-Blatchford (2001), "can grow up with the ability to retain their home language and culture, and to have pride in [themselves], as well as adapting to the new cultures and languages of any early childhood setting they encounter" (p.106). The insights provided in this study, based on its many findings, speak to this aim. 


\section{References}

Aboud, F.E., \& Mendelson, M. J. (1996). Determinants of friendship selection and quality: Developmental perspectives. In W. M. Bukowski, A. F. Newcomb, \& W. W. Hartup (Eds.), The company they keep: Friendship in childhood and adolescence (pp. 87-112). Cambridge: The Cambridge University Press.

Aboud, F. E., Mendelson, M. J., \& Purdy, K. T. (2003). Cross-race peer relations and friendship quality. International Journal of Behavioral Development, 27, 165 173.

Adair, J., \& Tobin, J. (2008). Listen to the voices of immigrant parents. In C. Genishi, \& A.L. Goodwin (Eds.), Diversities in early childhood education: Rethinking and doing (pp.137-150). New York: Routledge.

Adler, S. M. (2001). Racial and ethnic identity formation of mid-western Asian American children. Contemporary Issues in Early Childhood, 2(3), 265-294.

Alejandro, B., \& Celeste, R. (2001). Choice of languages in instruction: One language or two? Teaching Exceptional Children, 33(4), 10-16.

Alvestad, M. Duncan, J. \& Berge, A. (2009). New Zealand teachers talk about Te Whāriki. New Zealand Journal of Teachers' Work, 6(1), 3-19.

Alladina, S. (1995). Being bilingual: A guide for parents, teachers and young people on mother tongue, heritage language and bilingual education. Oakhill: Trentham Books.

Anderson, G. J. (1998). Fundamentals of educational research. New York: Falmer.

Anderson, R. T. (2004). Phonological acquisition in preschoolers learning a second language via immersion: A longitudinal study. Clinical Linguistics \& Phonetics, $18(3), 183-210$.

Anning, A., Cullen, J., \& Fleer, M. (Eds.). (2004). Early childhood education: Society and culture . London: Sage Publication.

Anning, A., Cullen, J., \& Fleer, M (Eds.). (2009). Early childhood education: Society and culture $\left(2^{\text {nd }}\right.$ ed.). London: Sage Publication.

Arnberg, L. (1987). Raising children bilingually: The pre-school years. Clevedon: Multilingual Matters.

Ary, D., Jacobs, L. C., \& Razavieh, A. (2002). Introduction to research in education $\left(6^{\text {th }}\right.$ ed.). Belmont, CA: Wadsworth/Thomson Learning.

Aubrey, C. (2000). Early childhood educational research: Issues in methodology and ethics. London: RoutledgeFalmer Press.

Baker, C. (1996). Foundations of bilingual education and bilingualism. Clevedon: Multicultural matters.

Baker, C. (2006). Foundations of bilingual education and bilingualism ( ${ }^{\text {nd }}$ ed.). Clevedon: Multilingual Matters.

Baker, C., \& Jones, S. P. (1998). Encyclopaedia of bilingualism and bilingual education. Clevedon: Multilingual Matters. 
Banks, J. A. (1988). Approaches to multicultural curriculum reform. Multicultural Leader, 1(2), 37-39.

Banks, J. A. (2001). Multicultural education: Goals, possibilities and challenges. In C.F. Diaz (Ed.), Multicultural education in the $21^{\text {st }}$ century (pp.11-22). New York: Longman.

Banks, J. A. (2006). Race, culture and education: The selected works of James A. Banks. New York: Routledge.

Banks, J A., \& Banks, C. (1993). Multicultural education: Issues and perspectives $\left(^{\text {nd }}\right.$ ed.). Boston: Allyn and Bacon.

Banks, J. A., \& Banks, C. (Eds.). (2006). Multicultural education: Issues and perspectives ( $6^{\text {th }}$ ed.). Hoboken: John Wiley \& Sons.

Barnard, R. (2003). Private speech in the mainstream classroom: Jack, a Korean learner. In R. Barnard, \& T. Glynn (Eds.), Bilingual children's language and literacy development (pp. 166-193). Clevedon: Multilingual Matters.

Baumeister, R.F., \& Leary, M.R. (1995). The need to belong: Desire for interpersonal attachments as a fundamental human motivation. Psychological Bulletin, 117(3), 497-529.

Beardsmore, H. B. (1986). Bilingualism: Basic principles $\left(2^{\text {nd }}\right.$ ed.). Clevedon: Multilingual Matters.

Bell, J. (2002). Doing your research project: A guide for first-time researchers in education and social science ( $3^{\text {rd }}$ ed.). Buckingham: Open University Press.

Bennett, C. I. (2006). Comprehensive multicultural education: Theory and practice (6 $^{\text {th }}$ ed.). Boston: Allyn and Bacon.

Bhatia, \& Ritchie (2008).The bilingual mind and linguistic creativity. Journal of Creative Communications, 3(1), 5-21.

Billman, N., Geddes, C., \& Hedges, H. (2005). Teacher-parent partnerships: Sharing understandings and making changes. Australian Journal of Early Childhood, 30(1), 44-48.

Blaikie, N.H. (1991). A critique of the use of triangulation in social research. Quality \& Quantity, 25(2), 115-136.

Bogdan, R. C., \& Biklen, S. K. (2003). Qualitative research for education: An introduction to theory and methods ( $4^{\text {th }}$ ed.). Boston: Allyn \& Bacon.

Brennan, M. A. (2005). "They just want to be with us." Young children learning to live the culture: A post-Vygotskian analysis of young children's enculturation into a childcare centre. Unpublished $\mathrm{PhD}$ thesis, Victoria University of Wellington, Wellington.

Brennan, M. A. (2006). Using socio-cultural approaches for educational research. Research and Policy Series 5. Wellington: Institute for Early Childhood Studies, Victoria University of Wellington.

Brewer, J. (2007). Introduction to early childhood education: Preschool through primary grades. Boston: Allyn \& Bacon. 
Brisk, M. (1998). Bilingual education: From compensatory to quality schooling. New Jersey: Lawrence Erlbaum.

Brooker, L. (2001). Interviewing children. In G. MacNaughton, S. A. Rolfe, \& I. SirajBlatchford (Eds.), Doing early childhood research: International perspectives on theory and practice (pp.162-177). NSW: Allen \& Unwin.

Brooker, L. (2002). Starting school: Young children learning cultures. Buckingham: Open University Press.

Brooker, L. (2005) Learning to be a child: Cultural diversity and early years ideology. In N. Yelland (Ed.), Critical issues in early childhood education (pp.115-130). Buckingham: Open University Press.

Broström, S. (2003). Understanding Te Whāriki from a Danish perspective. In J. Nuttall (Ed.), Weaving Te Whāriki: Aotearoa New Zealand's early childhood curriculum document in theory and practice (pp.215-238). Wellington: New Zealand Council for Educational Research.

Brown, D. M. (1979). Mother tongue to English: The young child in the multicultural school. Cambridge: Cambridge University Press.

Brown, J. D., Robson, G., \& Rosenkjar, P. (2001). Personality, motivation, anxiety, strategies and language proficiency of Japanese students. In Z. Dörnyei, \& R. Schmidt (Eds.), Motivation and language acquisition (pp.361-98). Honolulu: Second Language Teaching and Curriculum Center, University of Hawaii.

Brown, S.C., \& Kysilka, M. L. (2002). Applying multicultural and global concepts in the classroom and beyond. Boston: Allyn \& Bacon.

Brown, W. H., Odom, S.L., \& Buysse, V. (2002). Assessment of preschool children's peer-related social competence. Assessment for Effective Intervention, 27(4), 6171.

Bruner, J. (1996). The culture of education. Cambridge: Harvard University Press.

Bryman, A. (2004). Social research methods $\left(2^{\text {nd }}\right.$ ed.). Oxford: Oxford University Press.

Burman, E. (1994). Deconstructing developmental psychology. London: Routledge.

Callender, C. (1997). Education for empowerment: The practice and philosophies of black teachers. Staffordshire: Trentham Books.

Callister, P. (2004). Ethnicity measures, intermarriage and social policy. Social Policy Journal of New Zealand, 23,109-140.

Campbell, S., \& Page, J. (2003). Curriculum contexts: Becoming an early childhood professional. In G. MacNaughton (Ed.), Shaping early childhood education: Learners, curriculum and contexts (pp.282-301). Buckingham: Open University Press.

Canagarajah, A. (1995). Functions of codeswitching in ESL classrooms: Socializing bilingualism in Jaffna. Journal of Multilingual and Multicultural Development, 16(3), 173-195.

Carlson, S. M., \& Meltzoff, A. M. (2008). Bilingual experience and executive functioning in young children. Developmental Science, 11(2), 282-298.

Carr, M. (2001). Assessment in early childhood settings: Learning stories. London: Paul Chapman. 
Carr, M., \& May, H. (2000). Te Whāriki: Curriculum voices. In H. Penn (Ed.), Early childhood services: Theory policy and practice (pp.53-73). Buckingham: Open University press.

Carter, C, Minister of Education. (2008). Education (Early Childhood Education Curriculum Framework) Notice 2008. Wellington: Ministry of Education.

Chan, A. (2006). 'The teachers said my child is different'. The First years: New Zealand Journal of Infant and Toddler Education, 8(1), 34-38.

Chan, C. M. (2004).Issues of preschool parental education in Hong Kong in the twentyfirst century. Contemporary Issues in Early Childhood, 5(2), 257-263.

Chan, E. Y. M. (2004). Narratives of experiences: How culture matters of children's development. Contemporary Issues in Early Childhood, 5(2), 145-158.

Chan, S. (1999) The Chinese learner: A question of style. Education + Training, 41(67), 295-305.

Chan, S., \& Leong, C. W. (1994). Chinese families in transition: Cultural conflicts and adjustment problems. Journal of Social Distress and the Homeless, 3(3), 263-281.

Chang, H. C., \& Holt, G. (1994). A Chinese perspective on face as inter-relational concern. In S. Ting-Toomey (Ed.), The challenge of facework: Cross-cultural and interpersonal issues (pp.95-132). New York: State University of New York Press.

Chang, L., Li, K.K., Lei, L., Liu, H., Guo, B., Wang, Y., \& Fung, K. (2005). Peer acceptance and self-perceptions of verbal and behavioural aggression and social withdrawal. International Journal of Behavioral Development, 29(1), 48-57.

Chao, R.K. (1994). Beyond parental control and authoritarian parenting style: Understanding Chinese parenting through the cultural notion of training. Child Development, 65(4), 1111-1119.

Chao, R. K. (1995, August). Beyond Authoritarianism: A cultural perspective on Asian American parenting practices. Paper presented at the annual meeting of the American Psychological Association, New York.

Chavajay, P., \& Rogoff, B. (1999). Cultural variation in management of attention by children and their caregivers. Developmental Psychology, 35(4), 1079-1090.

Chen, F. M., \& Luster, T. (2002). Factors related to parenting practices in Taiwan. Early Child Development and Care, 172(5). 413-430.

Chen, H. (2001). Parents' attitudes and expectations regarding science education: Comparisons among American, Chinese-American, and Chinese families. Adolescence, 36(142), 305-314.

Chen, X., Rubin, K. H., \& Li, D. (1997). Relation between academic achievement and social adjustment: Evidence from Chinese children. Developmental Psychology, 33(3), 518-525.

Chen, X., Rubin, K. H., \& Sun, Y. (1992). Social reputation and peer relationship in Chinese and Canadian children: A cross-cultural study. Child Development, 63(6), 1336-1343.

Cheng, L.L. (1987). Assessing Asian language performance: Guidelines for evaluating limited-English proficient students. San Diego: Aspen Publishers. 
Chesterfield, R., \& Chesterfield, K. (1985). Natural order in children's use of second language learning strategies. Applied Linguistics, 6, 45-59.

Chiang, L. H. (2003, April). Learning social behaviors in families during childhood: A comparative study of Chinese children in Taiwan and in the United States. Paper presented at the Biennial meeting of the Society of Research in Child Development, Tampa.

Citizens Preschool and Nursery Centre of Innovation 2005-2007. (2008). Collaborations: Teachers and a family Whänau support worker in an early childhood setting. Wellington: Ministry of Education.

Clark, B. (2004). Self-talk or private speech in early childhood education: Complexities and challenges. New Zealand Research in Early Childhood Education, 7, 94-105.

Cobb, C., Danby, S., \& Farrell, A. (2005). Governance of children's everyday spaces. Australian Journal of Early Childhood, 30(1), 14-20.

Cole, M. (1988). Cross-cultural research in the sociohistorical tradition. Human Development, 31(3), 137-151.

Cole, M. (1998). Culture in development. In M. Woodhead, D. Faulkner, \& K. Littleton (Eds.). Cultural worlds of early childhood (pp.11-33). London: Routledge.

Cole, M. (2003, June).Vygotsky and context: Where did the connection come from and what difference does it make? Paper presented for the biennial conference of the International Society for Theoretical Psychology, Istanbul, Turkey.

Cole, M. (2005). Putting culture in the middle. In H. Daniels (Ed.), An introduction to Vygotsky (pp.199-226). London: Routledge.

Cole, M., \& Gajdamaschko, N. (2007). Vygotsky and culture. In H. Daniels, M. Cole, \& J. V. Wertsch (Eds.). The Cambridge companion to Vygotsky (pp. 193-211). Cambridge: Cambridge University Press.

Coplan, R. J., Prakash, K., O’Neil, K., \& Armer, M. (2004). Do you “want” to play? Distinguishing between conflicted shyness and social disinterest in early childhood. Developmental Psychology, 40 (2), 244-258.

Corrie, L. (1995). Vertical investigation: Teachers' knowledge and teachers' voice. Australian Journal of Early Childhood, 20(3), 1-5.

Corsaro, W. A. (1985). Friendship and peer culture in the early years. Norwood, NJ: Ablex Publishing Corporation.

Corsaro, W. A. (1997). The sociology of childhood. Thousand Oaks, CA: Pine Forge Press.

Corsaro, W. A. (2005). The sociology of childhood ( $2^{\text {nd }}$ ed.). Thousand Oaks, CA: Pine Forge Press.

Costigan, C. L., \& Dokis, D. P. (2006). The relations between parent-child acculturation differences and adjustment within immigrant Chinese families. Child Development, 77(5), 1252-1267.

Costigan, C. L., \& Su, T. (2004). Orthogonal versus linear models of acculturation among immigrant Chinese Canadians: A comparison of mothers, fathers, and children. International Journal of Behavioral Development, 28(6), 518-527. 
Cullen, J. (1996). The challenge of Te Whāriki for future developments in early childhood education, Delta, 48(1), 113-126.

Cullen, J. (2001). Ethics and assessment in early childhood programmes. Early Education, 27, 5-11.

Cullen, J. (2003). The challenge of Te Whāriki: Catalyst for change? In J. Nuttall (Ed.), Weaving Te Whāriki: Aotearoa New Zealand's early childhood curriculum document in theory and practice (pp.269-296). Wellington: New Zealand Council for Educational Research.

Cullen, J. (2008). Outcomes of early childhood education: Do we know, can we tell, and does it matter? Paper presented at the NZARE annual conference, Palmerston North, New Zealand.

Dahlberg, K., Drew, N., \& Nystrom, M. (2001). Reflective lifeworld research. Sweden: Studentlitteratur.

Dalli, C. (1997). Early childhood centres as parent support: A personal perspective. Childrenz Issues, 1(2), 21-24.

Dalli, C. (1998). Learning to be in childcare: Mother's stories of their child are 'settlingin'. European Early Childhood Education Research Journal, 7(2), 53-66.

Dalli, C. (1999). Starting childcare before three: Narratives of experience from a tri-partite focus. Unpublished $\mathrm{PhD}$ thesis, Victoria University of Wellington, New Zealand.

Dalli, C. (2000). Starting child care: What young children learn about relating to adults in the first weeks of starting child care. Early Childhood Research \& Practice, 2(2). Retrieved June 22, 2006, from http://ecrp.uiuc.edu/v2n2/dalli.html

Danaher, T., \& Briod, M. (2005). Phenomenological approaches to research with children. In S. Greene, \& D. Hogan (Eds.), Researching children's experience: Methods and approaches (pp. 217-235). London: Sage Publications.

Daniels, H. (Ed.) (1996). An introduction to Vygotsky. London: Routledge.

Daniels, H. (2001). Vygotsky and pedagogy. London: Routledge.

Daniels, H. (2005). An introduction to Vygotsky ( ${ }^{\text {nd }}$ ed.). London: Routledge.

Davidson, C., \& Tolich, M. (Eds.). (2003). Social science research in New Zealand:Many paths to understanding ( $2^{\text {nd }}$ ed.). Auckland: Pearson Education.

de Haan, D., \& Singer, E. (2001). Young children's language of togetherness. International Journal of Early Years Education, 9(2), 117-128.

de Melendez, R., \& Beck, W. (2007). Teaching young children in multicultural classrooms: Issues, concepts and strategies. New York: Thomson Delmar Learning.

DeBey, M., \& Bombard, D. (2007). Expanding children's boundaries: An approach to second-language learning and cultural understanding. Young Children, 62(2), 8893.

Deegan, J. G. (1991, April). An ethnography of children's friendships in a fifth-Grade culturally diverse class. Paper presented at the annual meeting of the American Educational Research Association, Chicago. 
Deegan, J. G. (1996). Children's friendships in culturally diverse classrooms. London: Falmer Press.

Denham, S., Mason, T., Caverly, S., Schmidt, M., Hackney, R., Caswell, C., \& DeMulder, E. (2001). Preschoolers at play: Co-socialisers of emotional and social competence. International Journal of Behavioural Development, 25(4), 290-301.

Denscombe, M., Szulc, H., Patrick, C., \& Wood, A. (1986). Ethnicity and friendship: The contrast between sociometric research and fieldwork observation in primary school classrooms. British Educational Research Journal, 12(3) 221-235.

Denzin, K. N., \& Lincoln, S. Y. (Eds.) (2003). The landscape of qualitative research: Theories and issues ( $2^{\text {nd }}$ ed.). California, Sage Publications.

Denzin, K. N., \& Lincoln, Y. S. (2005). Introduction: The discipline and practice of qualitative research. In K. N. Denzin, \& Y. S. Lincoln (Eds.), The Sage handbook of qualitative research ( $3^{\text {rd }}$ ed., pp.1-32). Thousand Oaks, CA: Sage.

Department of Labour (2009). Longitudinal immigration survey: New Zealand. Retrieved July 16, 2009, from http://www.immigration.govt.nz/migrant/general/generalinformation/research/lisn $\mathrm{z} /$

Dijkstra, S., Geuijen, K., \& de Ruijter, A. (2001). Multiculturalism and social integration in Europe. International Political Science Review, 22(1), 55-83.

Doddington, C., \& Hilton, M. (2007). Child-Centred Education: Reviving the creative tradition. London: Sage Publications.

Duncan, J. (2004). Misplacing the teacher? New Zealand early childhood teachers and early childhood education reforms, 1984-1996. Contemporary Issues in Early Childhood, 5(2), 160-177.

Dyson, L. (2001). Home-school communication and expectations of recent Chinese immigrants. Canadian Journal of Education, 26(4), 455-476.

Ebbeck, M., \& Gokhale, N. (2004). Child-rearing practices in a selected sample of parents with children in childcare in Singapore. Contemporary Issues in Early Childhood, 5(2), 194-206.

Education Review Office. (2004). Catering for diversity in early childhood services. Retrieved October 11, 2007, from http://www.ero.govt.nz/ero/publishing.nsf/Content/Reports+-+National+Reports

Edwards, A. (2005). Let's go beyond community and practice: The many meanings of learning by participating, The Curriculum Journal, 16(1), 49-65.

Ellis, R. (1985). Understanding second language acquisition. Oxford: Oxford University Press.

Ellis, R. (2002). The place of grammar instruction in the second/ foreign language curriculum. In E. Hinkel, \& S. Fotos (Eds.), New perspectives on grammar teaching in second language classrooms (pp.17-34). Mahwah: Lawrence Erlbaum.

Engeström, Y. (1987). Learning by expanding: An activity-theoretical approach to developmental approach. Helsinki: Orienta-Konsultit. 
Engeström, Y., Engeström, R. \& Kärkkäinen, M. (1995). Polycontextuality and boundary crossing in expert cognition: Learning and problem solving in complex work activities. Learning and Instruction, 5, 319-336.

Fang, G., Fang, F.X., Keller, M., Edelstein, W., Kehle, T.J., \& Bray, M.A. (2003). Social moral reasoning in Chinese children: A developmental study. Psychology in the Schools, 40(1), 125-138.

Faust, H., \& Smidt, S. (1998). 'I know lots of languages': Young bilingual children and their peers. In S. Smidt (Ed.), The early years: A reader (pp.60-77). London: Routledge.

Feden, P.D., \& Vogel, R.M. (2003). Methods of teaching: Applying cognitive science to promote student learning. New York: The McGraw-Hill Companies.

Feng, J.H. (1994). Asian-American children: What teachers should know. Retrieved October 30, 2005, from ERIC http://www.ericfacility.net/databases/ERIC_Digests/ed369577.html

Feng, S., Foo, S., Kretschmer, R., Prendeville, J., \& Elgas, P. M. (2004). Language and peer culture: Mandarin-speaking preschoolers in an English dominant setting. Language and Education, 18(1), 17-34.

Fillmore, L. W. (1976). The second time around: Cognitive and social strategies in second language acquisition. Unpublished Doctoral Thesis, Stanford University, Stanford, California.

Firth, A., \& Wagner, J. (2007). On discourse, communication, and (some) fundamental concepts in SLA research. Modern Language Journal, 91(5), 757-772.

Fleer, M. (2002) Socio-cultural theory: Rebuilding the theoretical foundations of early childhood education. Delta: Policy and Practice in Education, 54(1\&2), 105-120.

Fleer, M. (2003). Early childhood education as an evolving 'community of practice' or as lived 'social reproduction': Researching the 'taken-for-granted'. Contemporary Issues in Early Childhood, 4(1), 64-80.

Fleer, M., \& Robbins, J. (2004). Beyond ticking the boxes: From individual developmental domains to a sociocultural framework for observing young children. New Zealand Research in Early Childhood Education, 7, 23-39.

Fletcher, A. C., Bridges, T., \& Hunter, A. G. (2007). Managing children's friendships through interparental relationships: Roles of ethnicity and friendship context. Journal of Marriage and Families, 69, 1135-1149.

Flores, B. B., Keehn, S., \& Perez, B. (2002). Critical need for bilingual education teachers: The potentiality of "normalistas" and paraprofessionals. Bilingual Research Journal, 26(3), 501-524.

Florsheim, P. (1997), Chinese adolescent immigrants: Factors related to psychosocial adjustment. Journal of Youth and Adolescence, 26(2), 143-163.

Foner, N. (1999). The immigrant family: Cultural legacies and cultural changes. In C. Hirschman, P. Kasinitz, \& J. Dewind (Eds.), The handbook of international migration: The American Experience (pp.257-264). New York: Russell Sage Foundation. 
Foster, P., \& Ohta, A. S. (2005). Negotiation for meaning and peer assistance in second language classrooms. Applied Linguistics 26(3), 402-430.

Fumoto, J., Hargreaves, D., \& Maxwell, S. (2007). Teachers' perceptions of their relationships with children who speak English as an additional language in early childhood settings. Journal of Early Childhood Research, 5(2), 135-153.

Fung, H. (1999). Becoming a moral child: The socialization of shame among young Chinese children. Ethos, 27(2), 180-209.

Fürstenau, B. (2003). Exploration of an industrial expertise as a method of boundarycrossing in vocational education. In T.Tuomi-Grohn, \& Y. Engeström (Eds.), Between school and work: New perspectives on transfer and boundary-crossing (pp.85-118). Kidlington: Elsevier Science Ltd.

Gao, X. (2006). Strategies used by Chinese parents to support English language learning: Voices of 'elite' university students. Journal of Language Teaching and Research in Southeast Asia, 37(3), 285-298.

García, C.T., Lamberty, G., Jenkins, R., McAdoo, H. P., Crnic, K., Wasik, B. H., \& Vazquez García, H. (1996). An integrative model for the study of developmental competencies in minority children. Child Development, 67(5), 1891-1914.

Gay, G. (2000). Culturally responsive teaching: Theory, research, \& practice. Multicultural Education Series No. 8. New York: Teachers College Press.

Gay, L. R., Mills, G. E., \& Airasian, P. (2000). Educational research: Competencies for analysis and application (6 ${ }^{\text {th }}$ ed.). Upper Saddle River: Merrill.

Gerreiy, R. (2003). Responding to the cultural and linguistic diversity of refugee babies, toddlers and their families. The First Years: New Zealand Journal of Infant and Toddler Education 5(2), 33-37.

Gibbons, P. (2005). "The way we do things around here": Environmental and social considerations of the organisational culture of two playcentres. New Zealand Research in Early Childhood Education, 8, 11-27.

Gibson, M., Bejínez, LF., Hidalgo, N., \& Rolón, C. (2004). Belonging and school participation: Lessons from a migrant student club. In M. Gibson, P. Gándara, \& J. P. Koyama (Eds.), School connections: U.S.-Mexican youth, peers, and school achievement (pp.129-149). New York: Teachers College Press.

Giorgi, A. (1997). The theory, practice, and evaluation of the phenomenological method as a qualitative research procedure. Journal of Phenomenological Psychology, $28(2), 235-260$.

Glaser, B. G., \& Strauss, A. L. (1967). The discovery of grounded theory: Strategies for qualitative research. Chicago: Aldine.

Glass, B., Baker, K., Ellis, R., Bernstone, H., \& Hagan, B. (2008). Documenting for inclusion: How do we create an inclusive environment for all children? Early Childhood Folio. 12, 30-40.

Glynn, T. (2003). Responding to language diversity: A way forward for New Zealand education. In R. Barnard, \& T. Glynn (Eds.), Bilingual children's language and literacy development (pp.273-281). Clevedon: Multicultural matters.

Goldman, L. (1993). Misconceptions of culture and perversions of multiculturalism. Interchange, 24(4), 397-408. 
Gonzalez-Mena, J. (2008) Diversity in early care and education: Honoring differences. New York: McGraw-Hill.

Gonzalez-Mena, J., \& Bhavnagri, N. P. (2000). Diversity and infant/toddler caregiving. Young Children, 55(5), 31-35.

Goodnow, J. (1993). Direction of post-Vygotskian research. In E.A. Forman, N. Minick, \& C. A. Stone (Eds.), Contexts for learning: Sociocultural dynamics in children's development (pp.369-382). Oxford: Oxford University Press.

Gorman, C. J. (1998). Parenting attitudes and practices of immigrant Chinese mothers of adolescents. Family Relations, 41(1), 73-80.

Granger, C. A. (2004). Silence in second language learning: A psychoanalytic reading. Multicultural Series No. 6: Second Language Acquisition. Clevedon: Multilingual Matters.

Gravelle, M. (1996). Supporting bilingual learners in schools. England: Trentham Books.

Grey, A. (2003, September). Partnership with parents. Paper presented at the $8^{\text {th }}$ Early Childhood Convention, Palmerston North.

Grosjean, F. (1982). Life with two languages: An introduction to bilingualism. USA:The President and Fellows of Harvard College.

Gunn, A. C. (2000). Teachers' beliefs in relation to visual art education in early childhood centres. New Zealand Research in Early Childhood Education, 3, 153163.

Guo, K. (2005a). Asian Immigrant parents' and New Zealand early childhood teachers' views of parent-teacher relationships. New Zealand Research in Early Childhood Education, 8, 125-135.

Guo, K. (2005b). Developing in a new language-speaking setting. Australian Journal of Early Childhood, 30(3), 38-44.

Guo, Y. (2006). 'Why didn't they show up?' Rethinking ESL parent involvement in K12 education. TESL Canada Journal, 24(1), 80-95.

Guralnick, M. J., Connor, R.T., Hammond, M.A., Gottman, J. M., \& Kinnish, K. (1996). The peer relations of preschool children with communication disorders. Child Development, 67(2), 471-489.

Haggerty, M (1998). Sighting, citing and siting Te Whariki: Exploring the use of video feedback as a tool from critical pedagogy. Unpublished Master thesis, Victoria University, Wellington.

Hall, R. (2003). Forging a learning community?: A pragmatic approach to co-operative learning. Arts and Humanities in Higher Education, 2(2), 155 - 172.

Hall, S. (Ed.). (2003). Representation: Cultural representations and signifying practices (6 ${ }^{\text {th }}$ ed.). London: Sage Publications.

Hännikäinen, M. (1998). From togetherness to equal partnership in role play. Early Child Development and Care, 142, 23-32.

Hännikäinen, M. (2001). Playful actions as a sign of togetherness in day care centres. International Journal of Early Years Education, 9(2), 125-134. 
Hännikäinen, M., \& van Oers, B. (1999, September). Signs and problems of togetherness in a community of learners. Paper presented at the annual European Early Childhood Education Research Association Conference, Helsinki, Finland.

Hart, C. H., Yang, C., Nelson, J. L., Robinson, C., Olsen, J., Nelson, D. A., Porter, C. L., Jin, S., Olsen, S., \& Wu, P. (2000). Peer acceptance in early childhood and subtypes of socially withdrawn behaviour in China, Russia, and the United States. International Journal of Behavioral Development, 24(1), 73-81.

Hartup, W. W. (1996). The company they keep: Friendships and their developmental significance. Child Development, 67(1), 1-13.

Hatano, G. (1993). Time to merge Vygotskian and constructivist conceptions of knowledge acquisition. In E.A. Forman, N. Minick, \& C. A. Stone (Eds.), Contexts for learning: Sociocultural dynamics in children's development (pp.153168). Oxford: Oxford University Press.

Haworth, P., Cullen, J., Simmons, H., Schimanski, L., McGarva, P., \& Woodhead, E. (2006a). The role of acquisition and learning in young children's bilingual development: A sociocultural interpretation. International Journal of Bilingual Education and Bilingualism, 9(3), 295-309.

Haworth, P., Cullen, J., Simmons, H., Schimanski, L., McGarva, P., \& Woodhead, E. (2006b). The flight of our kite: The Wycliffe Nga Tamariki kindergarten story. Wellington: Ministry of Education.

Haworth, P., \& Haddock, D. (1999) Seeing NESB students with new eyes: Adjusting the teaching focus. Many Voices, 14, 7-15.

Hedegaard, M. (1990). The zone of proximal development as a basis for instruction. In L. Moll (Ed.), Vygotsky and education: Instructional implications and applications of sociohistorical psychology (pp.349-371). Cambridge: Cambridge University Press.

Hedges, H. (2003). Teaching and Learning: Theories that underpin 'wise' practice in Aotearoa/New Zealand. Early Education. 31, 5-12.

Hedges, H. (2007). Funds of knowledge in early childhood communities of enquiry. Unpublished PhD thesis, Massey University, Palmerston North, New Zealand.

Hellawell, D (2006). Inside-out: Analysis of the insider-outsider concept as a heuristic device to develop reflexivity in students doing qualitative research. Teaching in Higher Ed, 11, 483-494.

Hetherich, D., Hughes, P., Page, J., \& Campbell, S. (2003) Curriculum contexts: Critical reflections. In G. MacNaughton (Ed.), Shaping early childhood learners: Curriculum and contexts (pp.302-319). Buckingham: Open University Press.

Ho, E., Holmes, P., \& Cooper, J. (2004, May). Harmonizing diversities via emphasizing similarities: A study of multicultural classrooms in New Zealand. Paper presented at the third biennial international conference on intercultural research, Taipei.

Ho, M. (1998). Culture studies and motivation in foreign and second language learning in Taiwan. Language, Culture and Curriculum, 11(2), 165-182.

Hoel, T. L. (1999, September). Students cooperating in writing: Teaching, learning and research based theories from Vygotsky and Bakhtin. Paper presented at the European conference on educational research, Lahti, Finland. 
Hohnert, K., Yim, D., Nett, K., Kan, P, F., \& Duran, L. (2005). Intervention with linguistically diverse preschool children: A focus on developing home language(s). Language, Speech \& Hearing Services in Schools, 36(3), 251-264.

Hoogsteder, M., Maier, R., \& Elbers, E. (1998). Adult-child interaction, joint problem solving and the structure of cooperation. In M. Woodhead, D. Faulkner, \& K. Littleton (Eds.), Cultural worlds of early childhood (pp.178-195). London: Routledge.

Hoover-Dempsey, K. V., \& Sandler, H. M. (1997). Why do parents become involved in their children's education? Review of Educational Research, 67(1), 3-42.

Howes, C. (1996). The earliest friendships. In W. M. Bukowski, A. F. Newcomb, \& W. W. Hartup (Eds.), The company they keep: Friendship in childhood and adolescence (pp.66-86). Cambridge: Cambridge University Press.

Hruska, H. (2000, March). Bilingualism, gender, and friendship: Constructing second language learners in an English dominant kindergarten. Paper presented at the annual meeting of the American Association for Applied Linguistics, Vancouver, Canada.

Hu, C. (1944). The Chinese concepts of 'face'. American Anthropologist, 46(1), 45-64.

Hughes, C. E., Shaunessy, E.S., Brice, A. R., Ratliff, M.A., \& McHatton, P. A. (2006). Code switching among bilingual and limited English proficient students: Possible indicators of giftedness. Journal for the Education of the Gifted, 30(1), 7-28.

Hughes, P. (2003). Curriculum contexts: Parents and communities. In G. MacNaughton (Ed.), Shaping early childhood: Learners, curriculum and contexts (pp.255-281). Buckingham: Open University Press.

Hughes, P., \& MacNaughton, G. (2002). Preparing early childhood professionals to work with parents: The challenges of diversity and dissensus. Australian Journal of Early Childhood, 27(2), 14-20.

Huntsinger, C.S., Huntsinger, P.R., Ching, W. D., \& Lee, C.B. (2000). Understanding cultural contexts fosters sensitive caregiving of Chinese American children. Young Children, 55(6), 7-12.

Husserl, E. (1931/1977). Cartesian meditations: An introduction to phenomenology. The Hague: Martinus Nijhoff.

Hycner, R. (1985). Some guidelines for the phenomenological analysis of interview data. Human Studies, 8(3), 279-303.

Igoa, C. (1995). The inner world of the immigrant children. New Jersey: Lawrence Erlbaum Associates.

Ivic, I. (1989). Profiles of educators: Lev S. Vygotsky (1896-1934). Prospects: Quality Review of Education, XIX (3), 427-436.

Jain, A., \& J, Belsky. (1997). Fathering and acculturation: Immigrant Indian families with young children. Journal of Marriage and the Family, 59(4), 873-883.

Jambunathan, S., Burts, D., \& Pierce, S. (2000). Comparisons of parenting attitudes among five ethnic groups in the United States. Journal of Comparative Family Studies, 31, 395-406. 
Jaworski, A., \& Stephens, D. (2007). Self-reports on silence as a face-saving strategy by people with hearing impairment. International Journal of Applied Linguistics, $8(1), 61-80$.

Jenkins, H. (2006). Convergence culture: Where old and new media collide. New York: New York University Press.

John-Steiner, V., \& Tatter, P. (1983). An interactionist model of language development. In B. Bain (Ed.), The sociogenesis of language and human conduct (pp.79-97). New York: Plenum press.

Johnson, C., Ironsmith, M., Snow, C., \& Poteat, M. (2000). Peer acceptance and social adjustment in preschool and kindergarten. Early Childhood Education Journal, 27(4), 207-212.

Johnson, D .M. (1994). Grouping strategies for second language learners. In F. Genesee (Ed.), Educating second language children (pp. 183-211). Cambridge: Cambridge University Press.

Johnston, J., \& Wong, M-Y. (2002). Cultural differences in beliefs and practices concerning talk to children. Journal of Speech, Language and Hearing Research, $45,916-926$.

Jorgenson, J. N. (2003). Languaging among fifth graders: Code-switching in conversation 501 of the koge project. Journal of Multilingual and Multicultura, 24(1\&2), 126-148.

Jose, P. E., Huntsinger, C., Huntsinger, P., \& Liaw, F. (2000). Parental values and practices relevant to young children's social development in Taiwan and the United States. Journal of Cross-Cultural Psychology, 31(6), 677-702.

Joseph, J. (2004). Language and identity: National, ethnic, religious. Basingstoke: PalgraveMacmillan.

Kantor, R., Elgas, P., \& Fernie, D. E. (1998) Cultural knowledge and social competence within the preschool peer culture group. In M. Woodhead, D. Faulkner, \& K. Littleton (Eds.), Cultural worlds of early childhood (pp.133-154).London: Routledge.

Kaufman, H.O. (2001). Skills for working with all families. Young Children, 56(4), 8183.

Keats, D. M. (1997). Culture and the child: A guide for professionals in child care and development. England: John Wiley \& Sons Ltd.

Keddie, A. (2004). Research with young children: The use of an affinity group approach to explore the social dynamics of peer culture. British Journal of Sociology of Education, 25(1), 35-51.

Kemple, K. M. (2004). Let's be friends: Peer competence and social inclusion in early childhood programs. New York: Teachers College Press.

Klein, D., \& Chen, D. (2001). Working with children from culturally diverse backgrounds. New York: Delmar.

Kohnert, K., Yim, D., Nett, K., Kan, P. F., \& Duran, L. (2005). Intervention with linguistically diverse preschool children: A focus on developing home language(s). Language, Speech, and Hearing Services in Schools, 36(3), 251263. 
Konishi, C. (2007). Learning English as a second language: A case study of a Chinese girl in an American preschool. Childhood Education, 83(5), 267-272.

Krashen, S. (1982). Principles and practice in second language acquisition. New York: Pergamon Press.

Krapperman, L. (1996). The development of diverse social relationships in the social world of childhood. In A. E. Auhagen \& M. von Salisch (Eds.), The diversity of human relationships (pp.36-58). Cambridge: Cambridge University Press.

Krupa-Kwiatkowski, M. (1998). 'You shouldn't have brought me here!': Interaction strategies in the silent period of an inner-directed second language learner. Research on Language and Social Interactions, 31(2), 133-175.

Knafo, A., \& Plomin, R. (2006). Prosocial behaviour from early to middle childhood: Genetic and environmental influences on stability and change. Developmental Psychology, 42(5), 771-786.

Ladd, G. W. (1996). Shifting ecologies during the 5 to 7 year period: Predicting children's adjustment during the transition to grade school. In A. G. Sameroff \& M.M. Haith (Eds.), The five to seven year shift: The age of reason and responsibility (pp.363-385). Chicago: The University of Chicago Press.

Ladd, G.W., \& Kochenderfer, B. J. (1996). Linkages between friendship and adjustement during early school transitions. In W. M. Bukowski, A. F. Newcomb, \& W.W. Hartup (Eds.), The company they keep (pp.322-345). Cambridge: Cambridge University Press.

Lambert, W. (1973, November). Culture and language and factors in learning and education. Paper presented at the annual learning symposium on Cultural Factors in Learning, Bellingham, Washington. Retrieved on 2, March, 2007, from http://www.eric.ed.gov/ERICDocs/data/ericdocs2sq1/content_storage_01/0000019 b/80/37/fc/29.pdf

Lantolf, J. P. (2004). Overview of sociocultural theory. In O. St. John, K. van Esch, \& E. Schalkwijk (Eds.), New insights in second language learning and teaching (pp.13-34). Frankfurt: Peter Lang Verlag.

Lanza, E. (1997). Language mixing in infant bilingualism: A sociolinguistic perspective. Oxford: Oxford University Press.

Larkin, J. M., \& Sleeter, C.E. (Eds.). (1995). Developing multicultural teacher education curricula. Albany: SUNY Press.

Lau, S., Lew, W. J. F., Hau, K., Cheung, P. C., \& Berndt, T. J. (1990). Relations among perceived parental control, warmth, indulgence, and family harmony of Chinese in mainland China. Developmental Psychology, 26(4), 674-677.

Laubscher, L., \& Powell, S. (2003). Skinning the drum: Teaching about diversity as 'other'. Harvard Educational Review, 73(2), 203-224.

Lave, J., \& Wenger, E. (1991) Situated Learning. Legitimate peripheral participation, Cambridge: University of Cambridge Press.

Ledesma, H., \& Morris, R. (2005). Patterns of language preference among bilingual

(Filipino-English) boys. International Journal of Bilingual Education and Bilingualism, 8(1) 1-19. 
Lee, B., \& Chen, L. (2000). Cultural communication competence and psychological adjustment: A study of Chinese immigrant children's cross-cultural adaptation in Canada. Communication Research, 27(6), 764-792.

Lefkowitz, N., \& Hedgcock, J. (2002). Sound barriers: Influences of social prestige, peer pressure and teacher (dis)approval on FL oral performance. Language Teaching Research, 6(3), 223 - 244.

Leont'ev, Alexei N. (1981). Problems of the development of the mind. Moscow: Progress.

Li, G. (2006). Biliteracy and trilingual practices in the home context: Case studies of Chinese-Canadian children. Journal of Early Childhood Literacy 6(3), 355-381.

Li, J. (2001). Expectations of Chinese immigrant parents for their children's education: The interplay of Chinese tradition and the Canadian context. Canadian Journal of Education, 26(4), 477-494.

Li, J. (2004). 'I learn and I grow big': Chinese preschoolers' purposes for learning International Journal of Behavioral Development, 28(2), 116-128.

Li, J., \& Wang, Q. (2004). Perceptions of achievement and achieving peers in U.S. and Chinese Kindergartens. Social Development, 13(3), 413-435.

Lieberman, A. F. (1977). Preschoolers' competence with a peer: Relations with attachment and peer experience. Child Development, 48, 1277-1287.

Lin, C. C., \& Fu, V, R. (1990). A comparison of child-rearing practices among Chinese, immigrant Chinese and Caucasian-American parents. Child Development, 61(2), 429-433.

Lincoln, Y., \& Guba, E. (1985). Naturalistic inquiry. California: Sage Publications.

Lincoln, Y., \& Guba, E. (2000). Paradigmatic controversies, contradictions, and emerging confluences. In N. Denzin \& Y. Lincoln (Eds.), Handbook of qualitative research ( $2^{\text {nd }}$ ed., pp.163-188). California: Sage Publications.

Lindon, J. (2006). Equality in early childhood: Linking theory and practice. London: Hodder Arnold.

Liu, F. (2006). Yisha's first year of English acquisition. Language Arts, 83(4), 288-296.

Liu, M., Chen, X., Rubin, K. H., Zheng, S., Cui, L., Li, D., Chen, H., \& Wang, L. (2005). Autonomy- vs. connectedness-oriented parenting behaviours in Chinese and Canadian mothers. International Journal of Behavioral Development, 29(6), 489-495.

Lofdahl, A. (2006). Grounds for values and attitudes: Children's play and peer-cultures in pre-school. Journal of Early Childhood Research, 4(1), 77-88.

Long, S. (1997). Friends as teachers: The impact of peer interaction on the acquisition of a new language. In E. Gregory (Ed.), One child, many worlds: Early learning in multicultural communities (pp.123-136). New York: Teachers College Press.

Lubeck, S., Jessup, P., deVries, M., \& Post, J. (2001). The role of culture in program improvement. Early Childhood Research Quarterly, 16(4), 499-523.

Macartney, B. (2008). "If you don't know her, she can't talk": Noticing the tensions between deficit discourses and inclusive early childhood education. Early Childhood Folio, 12, 31-35. 
MacDonald, K. (1996). What do children want?: A conceptualisation of evolutionary influences on children's motivation in the peer group. International Journal of Behavioural Development, 19(1). 53-73.

MacNaughton, G. (2004). Children, staff and parents: Building respectful relationships in New Zealand and Australian early childhood contexts: The Australian context. Australian Journal of Early Childhood, 29(1), 1-7.

MacNaughton, G., \& Hughes, P. (2007). Teaching respect for cultural diversity in Australian early childhood programmes. Journal of Early Childhood Research, 5(2), 189-204.

Malcolm, K, T., Jensen-Campbell, L. A., Rex-Lear, M., \& Waldrip, A. M. (2006). Divided we fall: Children's friendships and peer victimization. Journal of Social and Personal Relationships, 23(5), 721-740.

Marsick, V., \& Kasl, E. (1997, May). Factors that affect the epistemology of group learning: A research-based analysis. Paper presented at the $38^{\text {th }}$ Annual Adult Education Research Conference, Oklahoma State University.

Martin, S. (2006). Opportunities for parent partnership and advocacy in early years services in Ireland. New Zealand Research in Early Childhood Education, 9, 1530 .

Marx, S. (2008). Popular white teachers of Latina/o kids: The strengths of personal experiences and the limitations of whiteness. Urban Education, 43(1), 29 - 67.

May, H. (2001). Politics in the playground: The world of early childhood in postwar New Zealand. Wellington: Bridget Williams.

May, S. (Ed.). (1999). Critical multiculturalism: Rethinking multicultural and antiracist education. London: Falmer press.

May, S. (2004). Accomodating multiculturalism and biculturalism in Aotearoa/New Zealand: Implications for language policy. In P. Spoonley, C. McPherson, \& D. Pearson (Eds.), Tangata, Tangata. The changing ethnic contours of Aotearoa/New Zealand (pp.247-264). Southbank: Thomson/Dunmore Press.

May, S., Modood, T., \& Squires, J. (2004). Ethnicity, nationalism and minority rights: Charting the disciplinary debates. In S. May, T. Modood, \& J. Squires (Eds.), Ethnicity, nationalism and minority rights (pp.1-26). Cambridge: Cambridge Press.

Maykut, P.S., \& Morehouse, R. E. (1994). Beginning qualitative research: A philosophic and practical guide. London: Falmer Press.

McDonald, G., Le, H. P., Higgins, J., \& Podmore, V. N. (2005). Artifacts, tools and classrooms. Mind, Culture, and Activity, 12(2), 113-127.

McNaughton, S. (2002). Meeting of minds. Wellington: Learning Media.

Merriam, S. B. (1988). Case study research in education: A qualitative approach. San Francisco: Jossey-Bass.

Merriam, S. B. (1998). Qualitative research and case study applications in education. San Francisco: Jossey-Bass.

Mickan, P. (2006). Socialization through teacher talk in an Australian bilingual class. International Journal of Bilingual Education and Bilingualism, 9(3), 342-358. 
Miles, M., \& Huberman, M. (1994). Qualitative data analaysis: An expanded sourcebook. London: Sage Publications.

Minick, N. (2005). The development of Vygotsky's thought: An introduction to thinking and speech. In H. Daniels (Ed.), An introduction to Vygotsky $\left(2^{\text {nd }}\right.$ ed., pp.33-58). New York: Routledge.

Minick, N., Stone, C.A., \& Forman, E.A. (1993). Introduction: Integration of individual, social and institutional processes in accounts of children's learning and development. In E.A. Forman, N. Minick, \& C.A. Stones (Eds.), Contexts for learning: Sociocultural dynamics in children's development (pp.IX-3). Oxford: Oxford University Press.

Ministry of Education (1993). Te Whāriki: Draft guidelines for developmentally appropriate programmes in early childhood services. Wellington: Learning Media.

Ministry of Education. (1996). Te Whāriki: He Whariki Matauranga mo nga Mokopuna o Aotearoa: Early childhood curriculum. Wellington: Learning Media.

Ministry of Education. (2002). Pathways to the future: Nga huarahi arataki: A 10 year strategic plan for early childhood education. Wellington: Ministry of Education.

Minisitry of Education. (2004). An introduction to Kei Tua o Te Pae: He whakimohiotanga ki. Kei Tua o te Pae. Kei Tua o te Pae: Assessment for learning: Early childhood exemplars, Book 1. Wellington: Learning Media.

Ministry of Education. (2004 \& 2005). Kei Tua o te Pae: Assessment for learning: Early childhood exemplars. Wellington: Learning Media.

Ministry of Education. (2008a). Number of enrolments in early childhood education by ethnicity. $\quad$ Retrieved October 19, 2008, from http://www.educationcounts.govt.nz/publications/ece/2507/27456/5

Ministry of Education. (2008b). Parents as first teachers. Retrieved March 31, 2008, from http://www.ecd.govt.nz/paft/how-works.html

Ministry of Education. (2008c). Education statistics of New Zealand 2007. Retrieved June 17, 2009, from http://www.educationcounts.govt.nz/publications/ece/2507/27456/27467/3

Ministry of Education. (2009a). Choices in early childhood education. Retrieved January 04, 2010, from http://www.minedu.govt.nz/Parents/EarlyYears/OtherInformationAndResources/ Choices/WhatAreMyChoices.aspx

Ministry of Education. (2009b). Enrolments in early childhood education. Retrieved February 12, 2010, from http://www.educationcounts.govt.nz/statistics/ece/55413/55414

Mitchell, L. (2003, September). Children staff and parents: Building respectful relationships in Australian and New Zealand early childhood contexts. Keynote address to the $8^{\text {th }}$ Early Childhood Convention, Palmerston North.

Mitchell, L. (2008). Early childhood education services in 2007: Key findings from the NZCER national survey. Retrieved July 3, 2009, from http://www.nzcer.org.nz/pdfs/16545.pdf 
Mitchell, L., \& Hodgen, E. (2008). Locality-based evaluation of pathways to the future: Ngā Huarahi Arataki. Phase 1 report. Wellington: Ministry of Education.

Modood, T., \& May, S. (2001). Multiculturalism and education in Britain: An internally contested debate. International Journal of Educational Research, 35(3), 305-317.

Moll, L. (1992). Bilingual classroom studies and community analysis. Educational Researcher, 21(2), 20-24.

Mooney, C. G. (2000). Theories of childhood: An introduction to Dewey, Montessori, Erikson, Piaget and Vygotsky. Minnesota: Redleaf Press.

Morrow, V., \& Richards, M. (2007). The ethics of social research with children: An overview. Children \& Society, 10(2), 90-105.

Mulder, S., Rance, S., Suarez, M., \& Condori, M. (2000). Unethical ethics? Reflections on intercultural research practices. Reproductive Health Matters, 8(15), 104-112.

Nakane, I. (2007). Silence in the multicultural classroom: Perceptions and performance. Amsterdam: John Benjamins.

Newcomb, A. F., \& Bagwell, C. L. (1996). The developmental significance of children's friendship relations. In W.M. Bukowski, A. F. Newcomb, \& W.W. Hartup (Eds.), The company they keep: Friendship in childhood and adolescence (pp.289-321). Cambridge: Cambridge University Press.

Newman, F., \& Holzman, L. (1993). Lev Vygotsky: Revolutionary scientist. London: Routledge.

New Zealand Teachers Council. (October, 2009). Registered Teacher Criteria. Retrieved December 29, 2009, from http://www.teacherscouncil.govt.nz/policy/projects2.stm

Ng, J. (1998). Social differences between Kiwi-Chinese and Chinese newcomers. Paper presented at the 1998 Chinese in Australia and the Pacific: Old and new migrations and cultural change meetings of the Association for the Study of Chinese and Their Descendants in Australasia and the Pacific Islands (ASCKAPI), Dunedin, New Zealand.

Nicoladis, E. (1998). First clues to the existence of two input languages: Pragmatic and lexical differentiation in a bilingual child. Bilingualism: Language and Cognition, $1(2), 105-116$.

Nilles, V., Alvarez, L., \& Rios, F. (2006). Preparing teachers to work with second language learners. In R. T. Jimenéz \& O. Pang (Eds.), Race, ethnicity, and education (pp.39-54). Westport: Praeger Publishing.

Nuttall, J. (2003). Exploring the role of the teacher within Te Whäriki: Some possibilities and constraints. In J. Nuttall (Ed.), Weaving Te Whāriki: Aotearoa New Zealands early childhood curriculum document in theory and practice (pp.161-186). Wellington: New Zealand Council for Educational Research.

Nuttall, J. (2005). Looking back, looking forward: Three decades of early childhood curriculum development in Aotearoa New Zealand. Curriculum Matters, 1, 12-18.

Ogbu, J. (1992). Understanding cultural diversity and learning. Educational Researcher, 21(8), 5-14. 
Okagaki, L., \& Diamond, K.E. (2000). Responding to cultural and linguistic differences in the beliefs and practices of families with young children. Young Children, 55(3), 74-80.

Osterman, K. (2000). Students' need for belonging in the school community. Review of Educational Research, 70(3), 323-367.

Ovando, C. J., Collier, V.P., \& Combs, M.C. (2002). Bilingual and ESL classrooms: Teaching in multicultural contexts. Boston: McGraw Hill.

Ozer, E., Wolf, J., \& Kong, C. (2008). Sources of perceived school connection among ethnically-diverse urban adolescents. Journal of Adolescent Research, 23(4), 438470.

Pachter, L. M., \& Dumont-Mathieu, T. (2004). Parenting in culturally divergent settings. In M. Hoghughi \& N. Long (Eds.), Handbook of parenting: Theory and research for practice (pp.88-97). London: Sage Publications.

Pacini-Ketchabaw, V., \& Schecter, S. (2002). Engaging the discourse of diversity: Educators' frameworks for working with linguistic and cultural differences. Contemporary Issues in Early Childhood, 3(3), 400-414.

Palmer, B. C., Chen, C., Chang, S., \& Leclere, J. (2006). The impact of biculturalism on language and literacy development: Teaching Chinese English language learners. Reading Horizons, 46(4), 239-265.

Parette, P., Chuang, S, J., \& Huer, M. (2004). First-generation Chinese American Families' attitudes regarding disabilities and educational interventions. Focus on Autism and Other Developmental Disabilities, 19(2), 114-123.

Parker, J. G., \& Gottman, J. M. (1989). Social and emotional development in a relational context: Friendship interaction from early childhood to adolescence. In T. J. Berndt \& G. W. Ladd (Eds.), Peer relationships in child development (pp.95132). New York: Wiley.

Patary-Ching, J., Kitt-Hinrichs, B., \& Nguyen, V. (2006). Inquiring into a second language and the culture of school. Language Arts, 83(3), 248-257.

Paulston, C. B. (1992). Sociolinguistic perspectives on bilingual education. Clevedon: Multilingual Matters.

Paulston, C. B., \& Tucker, G. R. (2003). Sociolinguistics: The essential readings. Malden: Blackwell Publishing.

Peirce, B. N. (1995). Social identity, investment, and language learning. TESOL Quarterly, 29(1), 9-31.

Persson, G. (2005). Developmental perspectives on prosocial and aggressive motives in preschoolers' peer interactions. International Journal of Behavioural Development, 29(1), 80-91.

Pfeifer, J., Rubble, D., Bachman, M., Alvarez, J., Cameron, J., \& Fuligni, J. (2007). Social identities and intergroup bias in immigrant and nonimmigrant children. Developmental Psychology, 43(2), 496-507.

Podmore, V. N., Samu, T. W., \& The Ao'oga Fa'aSamoa. A. (2006). O le tama ma lana a'oga, O le tama ma lona fa'asinomaga: Nurturing positive identity in children. Final Research Report from the A'oga Fa'a Samoa an early childhood centre of innovation. Wellington: Ministry of Education. Retrieved January 1, 2009, from 
http://www.educationcounts.govt.nz/_data/assets/pdf_file/0007/22588/11487finalresearchreport.pdf

Podmore, V. N., \& Sauvao, L. M., \& Mapa, L. (2003). Sociocultural perspectives on transition to school from Pacific Islands early childhood centres. International Journal of Early Years Education, 11(1), 33-42.

Portes, A., \& MacLeod, D. (1999). Educating the second generation: Determinants of academic achievement among children or immigrants in the United States. Journal of Ethnic and Migration Studies, 25(3), 373-396.

Powell, M, A., \& Smith, A. (2006). Ethical guidelines for research with children: A review of current research ethics documentation in New Zealand. Kötuitui: New Zealand Journal of Social Sciences Online, 1(2), 125-138. Retrieved March 21, 2009,

from http://www.royalsociety.org.nz/Site/publish/Journals/kotuitui/2006/09.aspx

Qualter, M., \& Munn, P. (2005). The friendships and play partners of lonely children. Journal of Social and Personal Relationships, 22(3), 379-397.

Quay, S. (1993). Language choice in early bilingual development. Unpublished PhD thesis, University of Cambridge, Cambridge.

Radice, H. (2008). Bridging identities?: Venice and the metaphor of the bridge. Paper presented at the International Studies Association annual conference, Retrieved May 14, 2009, from http://www.allacademic.com//meta/p_mla_apa_research_citation/2/5/2/9/8/pages2 52984/p252984-1.php

Rao, N., \& Yuen, M. (2001). Accommodations for assimilation: Supporting newly arrived children from the Chinese mainland to Hong Kong. Childhood Education, 77(5), 313-318.

Reeves, D. (2005). 'If I said something wrong, I was afraid'. Educating Language Learners, 62(4), 72-74.

Reyes, L. (2004). Functions of code switching in school children's conversations. Bilingual Research Journal, 28(1), 77-98.

Rhedding-Jones, J. (2002). An undoing of documents and other texts: Towards a critical multiculturalism in early childhood education. Contemporary Issues in Early Childhood, 3(1), 90-116.

Rhedding-Jones, J. (2005). Questioning diversity. In N. Yelland (Ed.), Critical issues in early childhood education (pp.131-145). Berkshire: Open University Press.

Ritchie, J. (2003) Whakawhanaungatanga: Dilemmas for mainstream New Zealand early childhood education of a commitment to bicultural pedagogy. Retrieved September, 27, 2009, from http://www.reconece.org/proceedings/ritchie_az2003.pdf

Rivera-Mills, S., \& Plonsky, L. (2007). Empowering students with language learning strategies: A critical review of current issues. Foreign Language Annals, 40(3), 535-549.

Robbins, J. (2002). Shoes and ships and sealing wax: Taking a sociocultural approach to interviewing young children. New Zealand Research in Early Childhood Education, 5, 13-30. 
Robbins, J. (2005). Contexts, collaboration and cultural tools: A sociocultural perspective on researching children's thinking. Contemporary Issues of Early Childhood, 6(2), 140-149.

Robinson, K. H., \& Jones-Díaz, C. (2006) Diversity and difference in early childhood education: Issues for theory and practice. Berkshire: Open University Press.

Robinson, P. (2001). Individual differences, cognitive abilities, aptitude complexes and learning cognitions in second language acquisition. Second Language Research, 17(4), 368-392.

Roer-Strier, D., \& Rosenthal, M, K. (2001). Socialization in changing cultural contexts: A search for images of the 'adaptive adult'. Social Work, 46(3), 215-228.

Rogoff, B. (1995), Observing sociocultural activity on three planes: Participatory appropriation, guided participation, and apprenticeship. In J. V. Wertsch, P. del Rio \& A. Alvarez (Eds.), Sociocultural studies of mind (pp.139-164). Cambridge: Cambridge University Press.

Rogoff, B. (1997). Evaluating development in the process of participation: Theory, methods and practice building on each other. In E. Amsel \& K. Renninger (Eds.), Change and development: Issues of theory, method and application (pp.265-285). Mahwah: Erlbaum.

Rogoff, B. (1998). Cognition as a collaborative process. In D. Kuhn \& R.S. Siegler (Eds.), Cognition, perception and language: Handbook of Child Psychology ( $^{\text {th }}$ ed, pp. 679-744). New York: Wiley.

Rogoff, B. (2003). The cultural nature of human development. New York: Oxford University press.

Rogoff, B. (2008). Observing sociocultural activity on three planes: Participatory appropriation, guided participation, and apprenticeship. In K. Hall, P. Murphy \& J. Soler (Eds.), Pedagogy and practice: Culture and identities (pp.58-74). London: Sage Publications.

Rogoff, B., \& Angelillo, C. (2002). Investigating the coordinated functioning of multifaceted cultural practices in human development. Human Development, 45, 211-225.

Rogoff, B., Matusov, E., \& White, C. (1996). Models of teaching and learning: Participation in a community of learners. In D. R. Olsen \& N. Torrance (Eds.), The handbook of education and human development (pp.388-414). Oxford: Blackwell.

Rogoff, B., Mistry, J., Göncü, A., Mosier, C., Chavajay, P., \& Heath, S. B. (1993). Guided participation in cultural activity by toddlers and caregivers. Monographs of the Society for Research in Child Development, 58 (8), 1-179.

Rogoff, B., Moore, L., Najafi, B., Dexter, A., Correa-Chavez, M., \& Solis, J. (2007). Children's development of cultural repertoires through participation in everyday routines and practices. In J. E. Grusec \& P. D. Hastings (Eds.), Handbook of socialization: Theory and research (pp.490-515). New York: Guilford.

Rogoff, B., Mosier, C., Mistry, J., \& Gőncü, A. (1993). Toddlers' guided participation with their caregivers in cultural activity. In E. A. Forman, N. Minick, \& C. A. 
Stone (Eds.), Contexts for learning: Sociocultural dynamics in children's development (pp.230-253). New York: Oxford University Press.

Rogoff, B., Mosier, C., Mistry, J., \& Gőncu, A. (1998). Toddlers' guided participation with their caregivers in cultural activity. In M. Woodhead, D. Faulkner, \& K. Littleton (Eds.), Cultural worlds of early childhood (pp.225-249). London: The Open University.

Rosenthal, D. A., \& Feldman, S. S. (1996). Crossing the border: Chinese adolescents in the West. In S. Lau (Ed.), Growing up the Chinese way: Chinese child and adolescent development (pp.287-320). Hong Kong: The Chinese University Press.

Russell, D. (2002). Looking beyond the interface: Activity theory and distributed learning. In M. R. Lea \& K. Nicoll (Eds.), Distributed learning: Social and cultural approaches to practice. London: The Open University.

Ryan, J. (1999). Race and ethnicity in multi-ethnic schools: A critical case study. Clevedon: Multilingual Matters.

Ryu, J. (2004). The social adjustment of three, young, high-achieving Korean-English bilingual students in kindergarten. Early Childhood Education Journal, 32(3), 165-171.

Salinas, M. S., Rance, S., Serrate, S. M., \& Castro, C. M. (2000). Unethical ethics? Reflections on intercultural research practices. Reprod Health Matters, 8(15), 104-112.

Saunders, G. (1988). Bilingual children: From birth to teens. Clevedon: Multilingual Matters.

Schnittker, J. (2002). Acculturation in context: The self-esteem of Chinese immigrants. Social Psychology Quarterly, 65(1), 56- 76.

Schofield, A. (2007). An investigation into the practices of a class of field-based student educators working in linguistically diverse early childhood centres. Australian Journal of Early Childhood, 32(2), 23-27.

Schultz, A., Walsh, G., \& Lehnert, F. (1967). The phenomenology of the social world. Illinois: Northwestern University Press.

Schwarzweller, H. K., \& Brown, J. S. (1962). Education as a cultural bridge between eastern Kentucky and the great society. Rural Sociology, 27(4), 357-353.

Scourfield, J., Dicks, D., Drakeford, M., \& Davies, A. (2006). Children, place and identity: Nation and locality in middle childhood. London: Routledge.

Sears, C. (1998). Second language students in mainstream classrooms: A handbook for teachers in international schools. Clevedon: Multilingual Matters.

Serpell, R. (1993). Interface between sociocultural and psychological aspects of cognition. In E.A. Forman, N. Minick, \& C. A. Stone (Eds.), Contexts for learning: Sociocultural dynamics in children's development (pp.357-368). Oxford: Oxford University Press.

Sharlin, S., \& Moin, V. (2001). New immigrants' perceptions of family life in origin and host cultures: In-group and out-group favoritism effect. Journal of Comparative Family Studies, 32(3), 405-418. 
Shek, D. (1996). The quality of family life and well being of Chinese parents in Hong Kong. Psychologia, 39(2), 84-93.

Shek, D., \& Chan, L.K. (1999). Hong Kong Chinese parents' perceptions of the ideal child. The Journal of Psychology, 133(3), 291-302.

Shi, X. (2006). Intercultural transformation and second language socialization. Journal of Intercultural Communication, 11, 2-16.

Silverman, D. (2000). Doing qualitative research: A practical handbook. London: Sage Publications.

Siraj-Blatchford, I. (2001). Diversity and learning in the early years. In D. G. Pugh (Ed.), Contemporary Issues in the early years ( $3^{\text {rd }} \mathrm{ed}$. pp.96-107). London: Paul Chapman.

Siraj-Blatchford, I., \& Clark, P. (2001). Supporting identity, diversity and language in the early years. Buckingham: Open University Press.

Skattebol, J. (2003). Dark, dark and darker: Negotiations of identity in an early childhood setting. Contemporary Issues in Early Childhood, 4(2), 149-166.

Sleeter, C. (1995). White preservice students and multicultural education coursework. In J. M. Larkin \& C. Sleeter (Eds.), Developing multicultural teacher education curricula (pp.17-30). Albany: State University of New York Press.

Sleeter, C., \& McLaren, P. L. (Eds.). (1995). Multicultural education, critical pedagogy, and the politics of difference. Albany: State University of New York Press.

Smith, A.B., Duncan, J., \& Marshall, K. (2005). Children's perspectives on their learning: Exploring methods. Early Child Development and Care, 175(6), 473487.

Souto-Manning, M. (2007). Immigrant families and children (re)develop identities in a new context. Early Childhood Education Journal, 34(6), 399-405.

Spolsky, B. (1969). Attitudinal aspects of second language learning. Language Learning, 19(3-4), 271-285.

Star, S.L., \& Griesemer, J. (1989). Institutional ecology, 'translations' and boundary objects: Amateurs and professionals in Berkeley's museum of vertebrate zoology, 1907-1939. Social studies of Science, 19, 387-420.

Statistics New Zealand. (2006). Profile of New Zealander responses, ethnicity question: 2006 Census. Retrieved October 16, 2007, from http://www.stats.govt.nz/analytical-reports/Profile+of+New+Zealander+ responses+ethnicity+question $+2006+$ Census.

Stewart-Mackenzie, H. (2007, September). How do NESB children and their families experience learning? Paper presented at the $9^{\text {th }}$ Early Childhood Convention, Rotorua, New Zealand.

Suarez-Orozco, M., \& Suarez-Orozco, C. (2000). Some conceptual consideration in the interdisciplinary study of immigrant children. In E. Trueba \& L. Bartolomé (Eds.), Immigrant voices: In search of educational equity (pp.17-36). Maryland: Rowman \& Littlefield. 
Suizzo, M. (2007). Parents' goals and values for children: Dimensions of independence and interdependence across four U.S. ethnic groups. Journal of Cross-Cultural Psychology, 38(4), 506-530.

Sumsion, J. (2003). Researching with children: Lessons in humility, reciprocity, and community. Australian Journal of Early Childhood, 28(1), 18-23.

Sumsion, J. (2005). Preschool children's portrayals of their male teacher: A poststructural analysis. In N. Yelland (Ed.), Critical issues in early childhood education (pp.58-81). Maidenhead: Open University Press.

Tabors, P. (1998). What early childhood educators need to know: Developing effective programs for linguistically and culturally diverse children and families. Young Children, 53(6), 20-26.

Tabors, P., \& Snow, C. (1994). English as a second language in preschool program. In J. Richards (Ed.), Educating second language children (pp. 103-126). Cambridge: Cambridge Press.

Tellis, W. (1997). Application of a case study methodology. The Qualitative Report, 3 (3). Retrieved August 23, 2007, from http://www.nova.edu/ssss/QR/QR33/tellis2.html

Terreni, L. \& McCallum, J. (2003). Providing culturally competent care in early childhood services in New Zealand. Part 1: Considering culture. Retrieved July 29, 2010, from http://www.eric.ed.gov/PDFS/ED475306.pdf

Tett, L. (2001). Parents as problems or parents as people? Parental involvement programmes, schools and adult educators. International Journal of Lifelong Education, 20(3), 188-198.

Thesing, A. (2008). Parent education and support through early childhood centre networks. New Zealand Research in Early Childhood Education, 11, 65-76.

Thorne, S. L. (2000). Second language acquisition theory and the truth(s) about relativity. In J. Lantolf (Ed.), Sociocultural theory and second language learning (pp.219-243). Oxford: Oxford University Press.

Todd, R. (1991). Education in a multicultural society. London: Cassell Educational.

Toma, J. D. (2006). Approaching rigor in applied qualitative research. In C. F. Conrad \& R. C. Serlin (Eds.), The Sage handbook for research in education (pp.405-423). London: Sage Publications.

Trnavsky, P. (1997). Play and cooperation: A longitudinal study of Chinese children. Early Child Development and Care, 138(1), 41-56.

Trumbull, E., Rothstein-Fisch, C., Greenfield, P., \& Quiroz, B. (2001). Bridging cultures between home and school: A guide for teachers with special focus on immigrant Latino families. Mahwah: Lawrence Erlbaum.

Tseng, W., Dörnyei, Z., \& Schmitt, N. (2006). A New approach to assessing strategic learning: The case of self-regulation in vocabulary acquisition. Applied Linguistics, 27(1), 78-102.

Ulich, M., \& Oberhuemer, P. (1997). Stories from two worlds: Bilingual experiences between fact and fiction. In E. Gregory, M. J. Drummond, \& A. Pollard (Eds.), One child, many worlds: Early learning in multicultural communities (pp.63-74). London: David Fulton Publishers. 
Van Manen, M. (1990). Researching lived experience: Human science for an action sensitive pedagogy. Albany: New York State University of New York Press.

van Oers, B. (2007). Helping young children to become literature: The relevance of narrative competence for developmental education. European Early Childhood Education Research Journal, 15(3), 299-312.

van Oers, B., \& Hännikäinen, M. (2001). Some thoughts about togetherness: An introduction. International Journal of Early Years Education, 9(2), 101-109.

Vedder, P., Horenczyk, G., Liebkind, K., \& Nickmans, G. (2006). Ethno-culturally diverse education settings: Problems, challenges and solutions. Educational Research Review, 1(2), 157-168.

Vygotsky, L.S. (1962). Thought and language. Cambridge: MIT Press.

Vygotsky, L. S. (1978). Mind in society: The development of higher psychological processes. Cambridge: Harvard University Press.

Vygotsky, L.S. (1981a). The genesis of higher mental functions. In J.V. Wertsch (Ed.), The concept of activity in Soviet psychology (pp.144-188). New York: Sharpe.

Vygotsky, L.S. (1981b). The instrumental method in psychology. In J.V. Wertsch (Ed.), The concept of activity in Soviet psychology (pp.134-143). New York: Sharpe.

Vygotsky, L (1986). Thought and Language. Cambridge: MIT Press.

Vygotsky, L. S. (1987). Thinking and speech. In R. W. Rieber \& A. S. Carton (Eds.), The collected works of L. S. Vygotsky: Vol. 1. Problems of general psychology (pp.39-285). New York: Plenum Press.

Vygotsky, L. S. (1994). The problem of the environment. In R. v. Veer \& J. Valsiner (Eds.), The Vygotsky reader (pp. 338-354). Oxford, UK: Blackwell Publishers.

Vygotsky, L. S. (1998). Infancy. In R. W. Rieber (Ed.), The collected works of L. S. Vygotsky: Vol. 5. Child psychology (pp.207-241). New York: Plenum Press.

Vygotsky, L., \& Luria, A. (1996). Tool and symbol in child development. In R. Veer \& J. Valsiner (Eds.), The Vygotsky reader (pp.99-174). Cambridge: Blackwell Publishers.

Wagner, H. (1970). Alfred Schutz: on Phenomenology and social relations. Chicago: The University of Chicago Press.

Wang, C., \& Pape, S. (2007). A probe into three Chinese boys' self-efficacy belief in learning English as a second language. Journal of Research in Childhood Education, 21(4), 364-377.

Wang, X. L., Bernas, R., \& Eberhard, P. (2005). Maternal teaching approaches in four cultural communities: Implications for early childhood teachers. Journal of Early Childhood Research, 3(3), 269-288.

Wang, Y., \& Ritchie, J. (2007). Comparing adults' and children's views on resolving children's conflict in China and New Zealand. Paper presented at the $9^{\text {th }}$ Early Childhood Convention, Rotorua, New Zealand.

Wartofsky, W. (1979). Models: Representation and scientific understanding. Holland: D. Reidel Publishing. 
Weinert, R. (1995). The role of formulaic language in second language acquisition: A review. Applied Linguistics, 16(2), 180-205.

Weisman, E.M., Flores, S.Y., \& Valenciana, C. (2007). Building bilingual bicultural learning communities: Experiences of Latino teacher candidate. Journal of Hispanic Higher Education, 6(3), 191-208.

Wells, G. (1993). Dynamics of discourse: Literacy and the construction of knowledge. In E. A. Forman, N. Minick, \& C. A. Stone (Eds.), Contexts for learning: Sociocultural dynamics in children's development (pp.58-90). Oxford: Oxford University Press.

Wenger, E. (1998). Communities of practice: Learning, meaning, and identity. Cambridge: Cambridge University Press.

Wenger, E. McDermott, R., \& Snyder, W. (2002). Cultivating communities of practice. Boston: Harvard Business School Press.

Wertsch, J. (Ed.). (1985). Culture, communication, and cognition: Vygotskian perspectives. Cambridge: Cambridge University Press.

Wertsch, J. (1991). Voices of the mind: A sociocultural approach in mediated action. USA: Harvard University Press.

Wertsch, J.V., Tulviste, P., \& Hagstrom, F. (1993). A sociocultural approach to agency. In E. Forman, N. Minick, \& C.A. Stone (Eds.), Contexts for learning: Sociocultural dynamics in children's development (pp.336-356). Oxford: OUP.

Wertsch, J.V., \& Tulviste, P. (1996). L.S. Vygotsky and contemporary developmental psychology. In H. Daniels (Ed.), An introduction to Vygotsky (pp.53-74). London: Routledge.

Wertsch, J. V., \& Tulviste, P. (2005). L.S Vygotsky and contemporary developmental psychology. In Daniels. H (Ed.), An introduction to Vygotsky (2 ${ }^{\text {nd }}$ ed., pp.59-80). London: Routledge.

West, M. (2001). Teaching the third culture child. Young Children, 56(6), 27-32.

White, C., Watts, N., \& Trlin, A. (2002). New Zealand as an English-language learning environment: Immigrant experiences, provider perspectives and social policy implications. Social Policy Journal of New Zealand, 18, 148-162.

Whitehead, M. (2007). Developing language and literacy with young child ( ${ }^{\text {rd }}$ ed.). London: Paul Chapman Publishing.

Willett, J. (1995). Becoming first graders in an L2: An ethnographic study of L2 socialization. TESOL Quarterly, 29(3), 473-503.

Woods, P., Boyle, M., \& Hubbard, N. (1999). Multicultural children in the early years: Creative teaching, meaningful learning. Clevedon: Multilingual Matters.

Woolfolk, A. (2004). Educational psychology. ( $9^{\text {th }}$ ed). Boston: Allyn \& Bacon.

Wu, B. (2009). "Whose culture has capital?”: Chinese skilled migrant mothers raising their children in New Zealand. Unpublished EdD thesis. Auckland University of Technology, Auckland.

Wu, C., \& Chao, R. (2005). Intergenerational cultural conflicts for Chinese American youth with immigrant parents: Norms of parental warmth and the consequences. International Journal of Behavioral Development, 29, 516-523. 
Xu, Y., Farver, J., Zhang, Z., Zeng, Q., Yu, L., \& Cai, B. (2005). Mainland Chinese parenting styles and parent-child interaction. International Journal of Behavioral Development, 29(6), 524 - 531.

Yau, J., \& Smetana, J. G. (2003). Conceptions of moral, social-conventional, and personal events among Chinese preschoolers in Hong Kong. Child Development, 74(3), 647-658.

Zadeh, Z, Y., Geva, E., \& Rogers, M. A. (2008). The impact of acculturation on the perception of academic achievement by immigrant mothers and their children. School Psychology International, 29(1), 39-70.

Zhang, C (2010). Who are Chinese?/ 谁是华人？华人是谁? Retreived July 25, 2010, from http://www.huayuqiao.org/articles/shcheong/shcheong02.htm.

Zhang, Y., Kohnstamm, G., Slotboom, A., Elphick, E., \& Cheung, P. C. (2002).

Chinese and Dutch parents' perceptions of their children's personality. Journal of Genetic Psychology, 163(2), 165-178.

Zwaans, A., van der Veen, I., Volman, M., \& ten Dam, G. (2008). Social competence as an educational goal: The role of the ethnic composition and the urban environment of the school. Teaching and Teacher Education, 24(8), 2118-2131. 
Appendices 
Appendix 1: Number of enrolments in early childhood education by ethnicity (Minisitry of Education, 2008a)

\begin{tabular}{|c|c|c|c|c|c|c|c|c|c|c|c|c|c|c|c|c|}
\hline \multirow[b]{2}{*}{ Ethnicity } & \multicolumn{2}{|c|}{2000} & \multicolumn{2}{|c|}{2001} & \multicolumn{2}{|c|}{2002} & \multicolumn{2}{|c|}{2003} & \multicolumn{2}{|c|}{2004} & \multicolumn{2}{|c|}{2005} & \multicolumn{2}{|c|}{2006} & \multicolumn{2}{|c|}{2007} \\
\hline & $\begin{array}{l}\text { Number } \\
\text { enrolled }\end{array}$ & $\begin{array}{c}\% \text { of } \\
\text { total } \\
\text { enrolled }\end{array}$ & $\begin{array}{l}\text { Number } \\
\text { enrolled }\end{array}$ & $\begin{array}{c}\% \text { of } \\
\text { total } \\
\text { enrolled }\end{array}$ & $\begin{array}{l}\text { Number } \\
\text { enrolled }\end{array}$ & $\begin{array}{c}\% \text { of } \\
\text { total } \\
\text { enrolled }\end{array}$ & $\begin{array}{l}\text { Number } \\
\text { enrolled }\end{array}$ & $\begin{array}{c}\% \text { of } \\
\text { total } \\
\text { enrolled }\end{array}$ & $\begin{array}{l}\text { Number } \\
\text { enrolled }\end{array}$ & $\begin{array}{c}\% \text { of } \\
\text { total } \\
\text { enrolled }\end{array}$ & $\begin{array}{l}\text { Number } \\
\text { enrolled }\end{array}$ & $\begin{array}{c}\% \text { of } \\
\text { total } \\
\text { enrolled }\end{array}$ & $\begin{array}{l}\text { Number } \\
\text { enrolled }\end{array}$ & $\begin{array}{c}\% \text { of } \\
\text { total } \\
\text { enrolled }\end{array}$ & $\begin{array}{l}\text { Number } \\
\text { enrolled }\end{array}$ & $\begin{array}{l}\% \text { of } \\
\text { total } \\
\text { enrolled }\end{array}$ \\
\hline NZ Māori & 32,255 & 18.5 & 31,026 & 18.1 & 32,779 & 18.7 & 33,892 & 18.8 & 35,232 & 19.1 & 35,756 & 19.3 & 35,000 & 19.0 & 35,618 & 18.7 \\
\hline Tokelauan & 410 & 0.2 & 366 & 0.2 & 373 & 0.2 & 338 & 0.2 & 324 & 0.2 & 325 & 0.2 & 273 & 0.1 & 272 & 0.1 \\
\hline Fijian & 365 & 0.2 & 360 & 0.2 & 379 & 0.2 & 395 & 0.2 & 414 & 0.2 & 476 & 0.3 & 461 & 0.2 & 448 & 0.2 \\
\hline Niuean & 657 & 0.4 & 634 & 0.4 & 693 & 0.4 & 755 & 0.4 & 826 & 0.4 & 794 & 0.4 & 721 & 0.4 & 669 & 0.4 \\
\hline Tongan & 2,083 & 1.2 & 2,201 & 1.3 & 2,159 & 1.2 & 2,187 & 1.2 & 2,230 & 1.2 & 2,166 & 1.2 & 2,062 & 1.1 & 2,112 & 1.1 \\
\hline Cook Island & 1,829 & 1.0 & 1,721 & 1.0 & 1,932 & 1.1 & 1,948 & 1.1 & 2,027 & 1.1 & 1,961 & 1.1 & 1,792 & 1.0 & 1,667 & 0.9 \\
\hline Samoan & 4,796 & 2.8 & 5,099 & 3.0 & 5,476 & 3.1 & 5,637 & 3.1 & 5,698 & 3.1 & 5,624 & 3.0 & 5,665 & 3.1 & 5,685 & 3.0 \\
\hline Other Pacific & 589 & 0.3 & 528 & 0.3 & 503 & 0.3 & 575 & 0.3 & 538 & 0.3 & 575 & 0.3 & 511 & 0.3 & 588 & 0.3 \\
\hline $\begin{array}{l}\text { Not Known } \\
\text { Pacifika }\end{array}$ & 12 & 0.0 & 8 & 0.0 & 4 & 0.0 & 3 & 0.0 & 5 & 0.0 & 6 & 0.0 & 7 & 0.0 & 193 & 0.1 \\
\hline Total Pasifika & 10,741 & 6.2 & 10,917 & 6.4 & 11,519 & 6.6 & 11,838 & 6.6 & 12,062 & 6.5 & 11,927 & 6.4 & 11,492 & 6.2 & 11,634 & 6.1 \\
\hline $\begin{array}{l}\text { South East } \\
\text { Asian }\end{array}$ & 875 & 0.5 & 858 & 0.5 & 892 & 0.5 & 1,056 & 0.6 & 1,009 & 0.5 & 1,042 & 0.6 & 1,074 & 0.6 & 1,152 & 0.6 \\
\hline Indian & 2,265 & 1.3 & 2,283 & 1.3 & 2,582 & 1.5 & 2,937 & 1.6 & 3,140 & 1.7 & 3,255 & 1.8 & 3,419 & 1.9 & 3,737 & 2.0 \\
\hline Chinese & 2,681 & 1.5 & 2,708 & 1.6 & 2,846 & 1.6 & 3,273 & 1.8 & 3,499 & 1.9 & 3,554 & 1.9 & 3,477 & 1.9 & 3,781 & 2.0 \\
\hline Other Asian & 2,124 & 1.2 & 2,421 & 1.4 & 2,579 & 1.5 & 2,620 & 1.5 & 2,817 & 1.5 & 2,778 & 1.5 & 2,812 & 1.5 & 3,074 & 1.6 \\
\hline Total Asian & 7,945 & 4.6 & 8,270 & 4.8 & 8,899 & 5.1 & 9,886 & 5.5 & 10,465 & 5.7 & 10,629 & 5.7 & 10,782 & 5.8 & 11,744 & 6.2 \\
\hline $\begin{array}{l}\text { Other (eg Sth } \\
\text { American, } \\
\text { African) }\end{array}$ & 2,571 & 1.5 & 2,261 & 1.3 & 2,469 & 1.4 & 2,549 & 1.4 & 2,712 & 1.5 & 2,855 & 1.5 & 3,026 & 1.6 & 5,943 & 3.1 \\
\hline $\begin{array}{l}\text { European/ } \\
\text { Pākehā }\end{array}$ & 120,733 & 69.3 & 118,859 & 69.4 & 119,297 & 68.2 & 121,835 & 67.7 & 124,043 & 67.2 & 124,360 & 67.0 & 124,154 & 67.3 & 125,968 & 66.0 \\
\hline TOTAL & 174,245 & 100 & 171,333 & 100 & 174,963 & 100 & 180,000 & 100 & 184,513 & 100 & 185,528 & 100 & 184,454 & 100.0 & 190,907 & 100.0 \\
\hline
\end{tabular}




\section{Appendix 2: Teacher participant information sheet}

\section{Letter head}

Dear teachers

My name is Karen GUO, a PhD Student at the Victoria University of Wellington. In order to complete my thesis I intend to look at the learning experiences of Chinese immigrant children in New Zealand early childhood centres.

As a person from an immigration background myself, I am interested in knowing whether these children's home learning experiences impact on their learning and development in New Zealand early childhood centres and if so, how and to what extent.

In the study that I am now undertaking, my aim is to gather information on what differences occur on rearing and educating young children between Chinese immigrant families and New Zealand early childhood settings. In particular, I will be describing these children's learning experiences in New Zealand childcare centres and identifying how they can be supported towards a positive learning experience.

The research has the approval of the Faculty of Education, Ethics Committee at the Victoria University of Wellington. It is being supervised by Doctor Val Podmore and Doctor Carmen Dalli in the School of Education Studies.

I propose to study Chinese immigrant children in your centre. I prefer the child/ren aged from three to five who speak Mandarin Chinese as the first language. Their parents should have been here for no more than ten years. I will gather information by observing each child and interviewing the child, some teachers and the child's parents. My observations with a child are expected to carry out for one day a week for five weeks. My role will be that of an 'impartial observer', that is, I would stay in a distance from the child most of the time and interact with the child occasionally. I will take care not to interrupt the child's play in the programme.

An interview will be conducted with the child in the centre towards the end of my observations of the child when I notice the child playing alone. This will happen as conversations between us. I will focus on the child's opinions of early childhood education at home and in the centre and the interview is proposed to be completed in half an hour. The child will be guided to express opinions through both verbal and nonverbal means, such as drawing or role play.

Semi-structured interviews with teachers and the parents will be conducted too. Interviews will be audio-taped. Questions would be asked to teachers and parents for information about the child's learning experiences and needs as well as teachers and parents' opinions of raising and educating Chinese immigrant children. Parents' interviews will be conducted in Mandarin.

Informal interviews/conversations will be conducted on an ongoing process to clarify some issues and minimise omissions of the observations. The interviews would not be used to elicit criticism of anyone involved. They would be analysed to get in-depth information about the studied child. Upon completion of the interviews, notes taken will 
be passed on to you for feedback. A summary of the findings will be provided for both teachers and parents at the completion of the data collection.

The information collected will be kept in a strictly confidential manner and the only other people with access to any information are my supervisors Dr Val Podmore and Dr Carmen Dalli. Moreover, all names used will be fictitious and confidentiality is assured at all times. I will make sure that the information is kept anonymous. Although the study process or results may be published in academic journals and the collected information may be disseminated at professional conferences, the names of anybody involved in this study will not appear in any publications. The data collected from the study will be destroyed at the conclusion of the research. You have the right to participate and withdraw from the study at any time until the end of May 2007.

I would appreciate it if you would approve your participation in this research. Once this approval has been granted, I would explore with you which child/ren at the centre might be appropriate for such a study. I would then seek the permission of the parents or guardians concerned, subject to the centre's approval. I also hope to interview some of the teachers at the centre in terms of their perceptions of the child and their expectations and working experiences with Chinese immigrant children. If you have any queries or would like to know more about the study please do not hesitate to contact me at ...

Thank you for your cooperation and I look forward to hearing from you.

Karen Guo 


\section{Appendix 3: Parent participant information sheet}

\section{Dear Parent/s}

My name is Karen Guo, a PhD student at the Victoria University of Wellington. I wish to conduct a research on how your child's learning experience in an early childhood centre in New Zealand.

As a person who also came from the Chinese cultural background, and as someone who is involved in early childhood education, I am interested in knowing whether the different childrearing beliefs and approaches between Chinese immigrant families and New Zealand childcare institutes impact on the learning and development of these children and if so, how. In particular, I wish to look at your child's interests, strengths and needs. My observation will be carried out for five weeks, one day a week. I will take care not to interrupt her/his play. The aim is not to critique your child in any way; it is simply to observe how your child communicates, plays, learns and interacts with others. I also hope to be able to interview you concerning your own perceptions about your child's involvement in the early childhood centre during this study. Your expectations and needs will be invited in a hope of finding out your preferred ways of raising young children. It is my plan too, to interview your child at the centre for about half an hour to understand her/his own perceptions of the learning experiences in your family and in the early childhood centre.

In the final document imaginary names will be used and confidentiality will be maintained throughout the study. The only other people with access to any information will be my supervisors, Dr Val Podmore and Dr Carmen Dalli of the Faculty of Education, Victoria University of Wellington. I am expecting to meet you in person for some of your ideas about the studied issue. Upon completion of the interviews, notes taken will be passed on to you for feedback. The research results may be published in academic journals or disseminated in professional conferences but confidentiality will be maintained in any situations. The collected data will be destroyed at the conclusion of the study. You have the right to participate and withdraw from the study at any time until the end of May 2007. After the completion, I will provide you with a summary of the findings.

I would appreciate it if you would approve your child's participation in this research. If you have any queries or would like to know more about the study please don't hesitate to contact me at ...

Thank you for your cooperation.

Karen Guo 


\title{
Appendix 4: Consent forms for teachers and parents
}

\author{
VICTORIA UNIVERSITY OF WELLINGTON \\ FACULTY OF EDUCATION
}

\section{Research title:}

Chinese immigrant children in New Zealand childcare settings

Student Name: Karen Liang Guo

Supervisors: Val Podmore \& Carmen Dalli

I have read the information about this study and have had the details of the study explained to me.

I understand that the research procedures will be as follows:

1. One interview, which will be transcribed. It is to request our ideas about the child, the studied issues and our parental/teaching experiences with the studied child/ren.

2. Transcripts and research discussion draft corrections by the participant. A summary of the research will be provided at my request.

3. Follow up discussion.

4. Child observations in narrative forms. The children's play and learning individually, with peers and with teaches will be observed and details will be noted down.

5. An interview with the child. The child will be guided to express their opinions of the learning experiences at home and in the childcare centre in verbal and nonverbal means.

6. The results may be disseminated at academic or professional conferences or published in academic or professional journals but our names will not appear anywhere and no information will be attributed to me in any way that will identify me.

I also understand that I am free to withdraw the study at any time, or to decline to answer any particular questions in the study. I agree to provide information to the researcher on the understanding that it is completely confidential.

I am willing to allow the study to proceed in the childcare centre under the conditions set out on the letter.

I am willing to participate in this study and happy to have my child participated in this study (parents)

Signed

Date: 


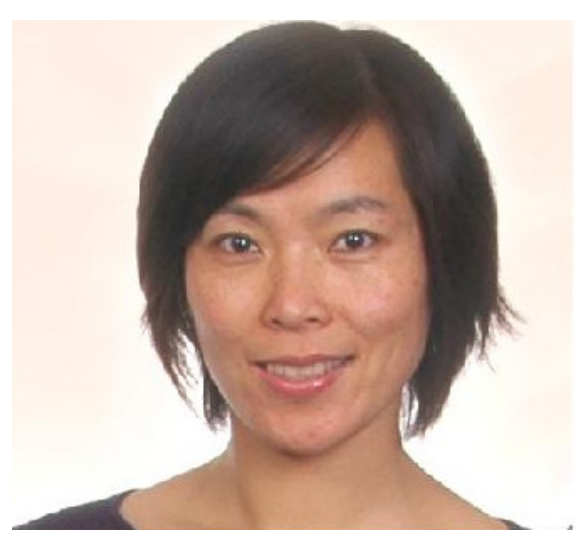

Title of research: Chinese immigrant children in New Zealand childcare centres

Researcher's name: Karen Guo

The Child's name:

I know why Karen is at my centre.

Yes/No (please circle one)

It is ok/not ok (please circle one) that she watches me at my centre.

It is ok/not ok (please circle one) that she takes photos and videotapes me playing.

It is ok/not ok (please circle one) that I talk to her about myself, my friends and my teachers at my centre and my mum or dad at my home.

I am

(please circle one) that Karen comes to my centre one day every week for five weeks to do these things. 


\section{Appendix 6: Children's interview schedule}

Date of interview

Childcare Centre

Name of the interviewee

Total time of interview

The interviews with each child will be conducted using pictures and some story beginnings. The procedures followed in conducting these interviews are:

1. approach the child

2. play with the child

3. tell the child 'Let me tell you a story about Hong (the child in the pictures). Show each picture for one minute then narrate the standard story beginning

4. take the picture away and ask the child to continue the story.

The pictures display the scenarios reflecting the child's learning experiences in five ways. These story beginnings include:

-having a day at home

-going to childcare in the morning

-free play

-playing with peers

-playing with a teacher/teachers

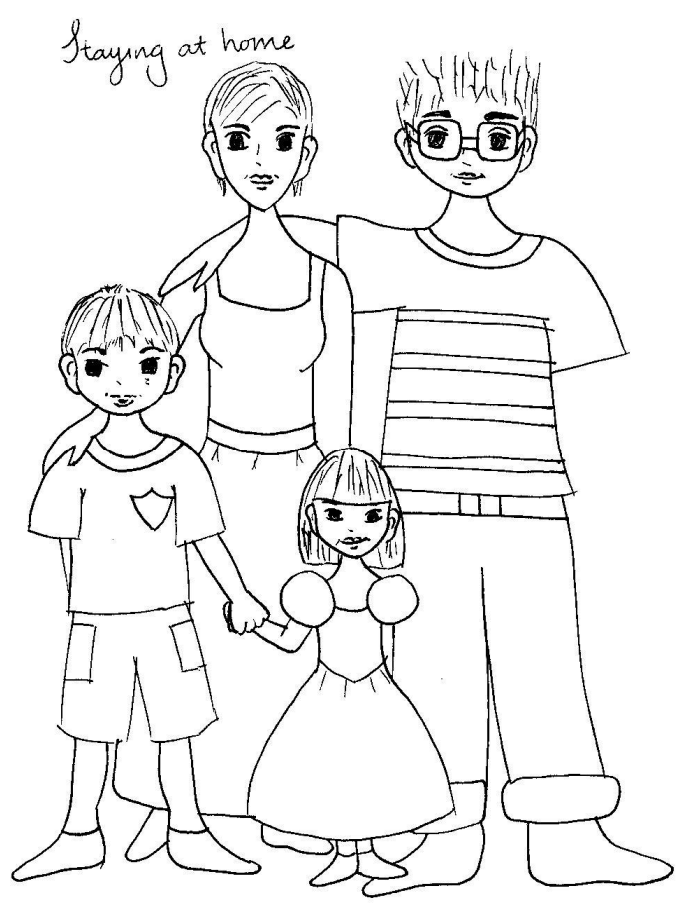



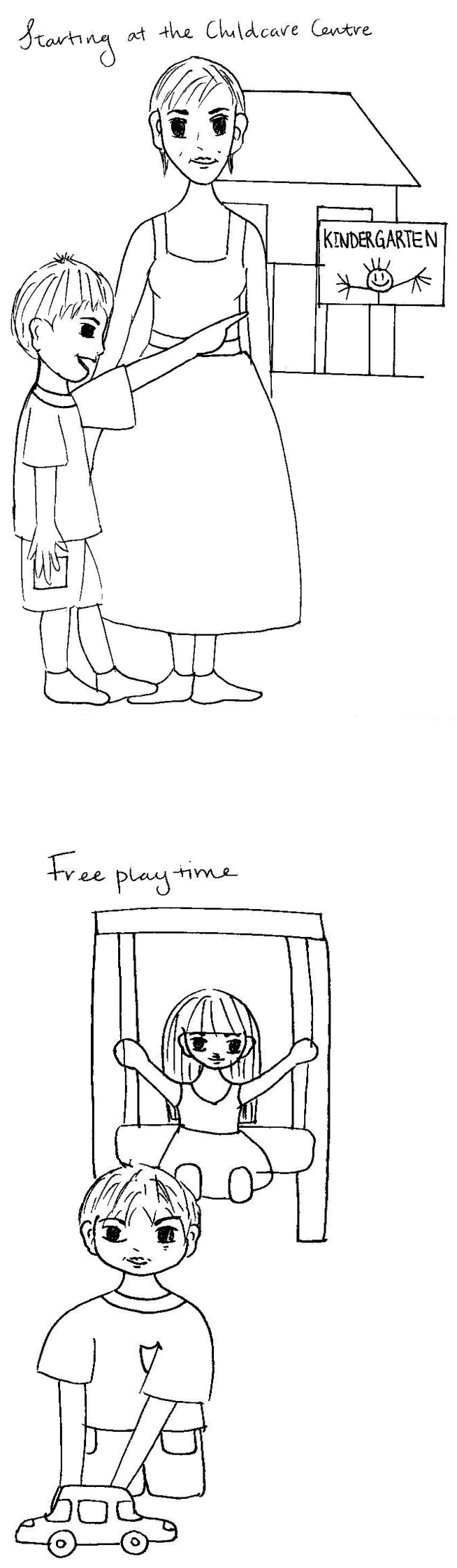
playing with peevs

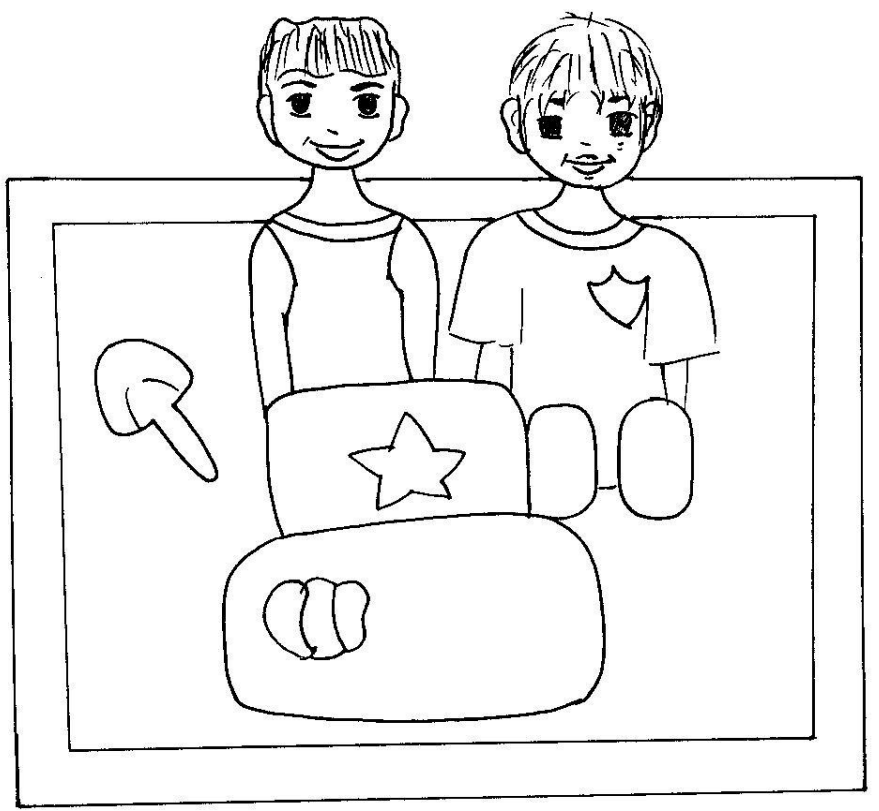

Playing with a teacher

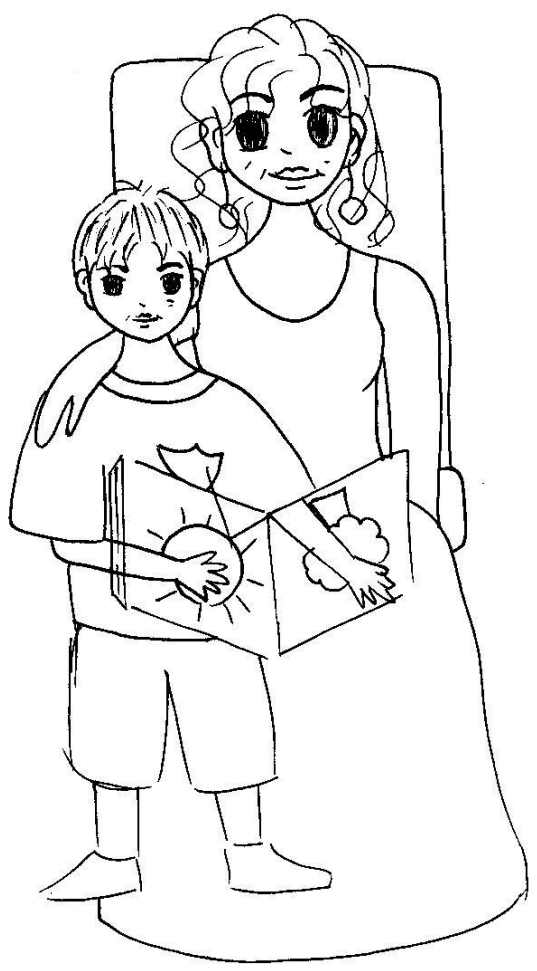




\section{Appendix 7: Parents' interview schedule}

\section{Research Title: Chinese Immigrant Children in New Zealand Childcare Centres}

\section{Date of interview}

Sex of the interviewee

Relationship to the child

Length of staying in New Zealand

Educational background of the interviewee

Family structure of the interviewee

Name of the child

Length of attendance of the child at the centre

Total time of interview
Childcare Centre

Type of Centre

Age of the interviewee

Place of birth of the interviewee

Age of the child

1. Background information of the parent

Can I know some information about your education, profession, family structure and opinions of your immigration experiences so far?

2. Background information of the child

How would you describe (the child's name)-such as his/her strengths and interest?

Can you describe the learning process of your child over the past years?

Does you child have any special needs?

2. Social experiences of the child

Did your child have any friends before coming here?

Do you know if the child has any friends now?

How does he/she play with others, children and adults?

3. Language learning experiences of the child

Did your child know any English before coming here? If so, how much do you think he/she knew?

What language do you usually speak with your child? How often do you speak this language?

Does anybody in your home speak English to your child? If so, when? Where? In what contexts?

4. Home learning experiences

What is your child's typical day routine at home during the weekdays or at weekend? 
What do you tend to do with your child?

Do you maintain the Chinese culture with your child? If so, why and how?

Have your experiences as an immigrant made an influence on your parental roles?

\section{Centre experiences}

Had your child been to any other childcare places in either New Zealand or in your own country before coming to this centre?

Have your child changed in any way after coming here? If so, in what ways?

Do you know whether your child can use English here?

How does your child understand being at a place out of home like this?

Now, please if you have any, tell me your general opinions of this childcare centre.

6. General impression of the centre

What opinions do you have about the teachers here in terms of their work with your child?

Do you think an environment like this centre helps a lot with your child's learning and development?

What's your opinion of New Zealand education as opposed to the education in your country of origin?

7. Parent involvement in the centre

Have you learnt about the teaching approaches with children in general and your child in particular?

Do you express your concerns about your child to the teachers here? If so, when and to whom? If not, why?

Are you comfortable/confident to approach teachers? Why?

Do teachers inform you of your child's experience in the centre and ask you for your opinion? If so, how often and when?

Are there any specific things in your child's centre that concern you?

How would you like to work with teachers?

The following questions will be related to your expectations of your child, 


\section{Parent expectations}

In what way do you think children learn and develop?

Which is the most important aspect of children's development to you?

What made you send your child to a childcare centre and this centre?

What in particular do you want your child to learn/develop at this centre?

What are your expectations and aspirations for your child's learning/education and development?

Is there anything else you would like to say about your child, the child's learning experiences at home and at this centre? 


\section{Appendix 8: Teachers' interview schedule}

\section{Research title: Chinese Immigrant Children in New Zealand Childcare Centres}

Date of interview

Sex of the interviewee

Position at the centre of the interviewee

Ratio, nationality diversity of the centre

Name of the child

Working period of the interviewee at the centre

Period of attendance of the child at the centre

Total time of interview
Childcare Centre

Type of Centre

Place of birth of the interviewee

1. Background of the Teacher

How long have you been working in this centre?

How long have you been involved in early childhood education?

What formal early childhood qualifications do you have?

2. What are the general expectations of children's learning experiences here?

How do you think young children develop?

How is the programme implemented at the centre?

3. Centre's teaching approach with immigrant children

What are Chinese immigrant children like in general?

Have you ever encountered difficulties when working with immigrant children? If so, what were they?

What do you think are the main learning needs of immigrant children?

What factors contribute to successful settlement of immigrant children?

Are there any behaviours from Chinese immigrant parents and children that you think are obviously distinct from what you are familiar with?

Do you use any special approaches to help immigrant children?

4. The learning experiences of the studied child

How long has (the child's name) been in this centre? 
Are there any particular activities that the child does not like to do?

Does (the child's name) have friends? Have you noticed how he/she usually behaves in groups?

How do you feel about the child's English?

Can you describe the child's settlement process?

How have you been supporting the child?

5. Parents' involvement

What learning experiences do you know (the child's name) has at home?

Do the child's parents get involved in your centre's programme? If so, how often and when?

Have you ever talked with parents about their ideas of their child's early education? If so, when and how often?

What have you been doing to help immigrant parents participate in the programs?

6. Further comments or information about the topic 


\section{Appendix 9: Teachers and parents' interview transcript feedback}

\section{To the teachers}

(Date)

Dear

This is the transcript of our interview which was conducted on You have the right to correct, add, or eliminate parts of the interview. If you make some corrections, I will send you the updated transcript for approval. If I have not heard from you by I will assume that you are comfortable for me to use the data from the interview.

I look forward to hearing from you. 


\section{Appendix 10: An individual case description}

The experiences of each participating child were analyzed and described before the data were cross analyzed and presented as findings. I include an example of an individual case description here: Amy's experience.

\section{Amy's Experience}

\section{Introducing Amy}

Amy was chosen by the centre manager because "She is a typical Chinese little doll" (informal teacher discussion). She presented to others as a happy, bright and friendly child, with a ready smile and a strong love of singing and dancing. Amy appeared to be close to the teachers and to "love being with adults" (teacher interview). For her parents, "she was flexible and was able to entertain herself and others quite easily" (parent interview).

Amy was three years and six months and had been at the centre for four months when my research began. Although she was mostly quiet, she did speak at times. Amy was born in New Zealand, into a Mandarin-Chinese speaking home, and was a proficient Mandarin speaker. Amy's parents came from Mainland China and had been living in New Zealand for six years. Her father was a full time computer engineer, and her mother was a part-time accountant. She also had a big sister who was two years older than her. Amy's typical day at the childcare centre was from 8:30am to 5:30pm. Her father usually dropped her off and collected her.

Amy's mother took care of her at home before she turned 18 months. Then, while her father remained in New Zealand, her mother returned with Amy and her sister to China for one and half years, where she attended a childcare centre for a year. They returned to New Zealand when Amy was three years old because her sister needed to start school. Amy attended a childcare centre for two months before changing to the present centre. "We [her parents] decided to move her out of there because she cried too much and was very unhappy. There was a vacancy in this centre, so we put her here" (parent interview). 
During my study, Amy initiated few contacts with me but did not hesitate to respond to me when approached. Prior to my arrival, her mother gave her the assent form and we met for a short talk, but she did not seem to pay any special attention to me during my stay. When we talked during the interview, Amy was fond of sharing with me her childcare experience. For her, there is no way that she could not come to childcare "because everybody else comes to childcare" (child interview). Amy loved her childcare experiences because "children play there" (child interview) and she wants to play (child interview).

Amy's centre was a privately owned childcare centre, catering for children from two to five years of age. The centre is divided into two rooms, one for over four year olds and the other for 2 to 4-year-olds, which was Amy's room. The two groups were managed separately but the children mingled during outside free-play times. During my study, they played outside for all of the afternoons and about one third of their morning sessions. Children were from many cultural groups and there were five Chinese children in the centre and two in Amy's group. There were 45 children on the roll and seven fulltime teachers. Three teachers and 20 children were in Amy's room. The teaching and learning programme at the centre was based on children's interests and guided by $T e$ Whäriki. According to the centre manager, "the centre embraced the style of teacherguided free play”. Maria was a trained primary school teacher, Iby was a qualified early childhood teacher, and Fiona was in training at an early childhood tertiary institution.

\section{English language in daily conversations}

\section{Amy's English language profile}

Amy managed many routines that required English. When there was a need to use it, she was either quiet or made a simple expression and quite often resorted to body language to help her express herself. Quite often, she smiled, nodded, shook her head or pointed to relevant places or objects. . Except for the use of these strategies, Amy's data revealed that in daily conversations, she weighed up the situations to make language choices. Amy was also observed to attempt to learn the new language. 


\section{Using languages in daily conversations}

Speaking Chinese when being alone, with Ken, a Chinese peer, or with Karen, a Chinese researcher

Amy was able to make correct language choices according to the contexts and people she was with, so she spoke English with the English speaking people, such as her teachers and the English speaking peers, and only Chinese to herself, with Ken, a Chinese boy or with the people who could speak Chinese. The following examples illustrate Amy's use of Chinese in some situations.

Amy runs with a ball: 'stop, stop, wo rang ni stop [stop, stop, I tell you to stop].

(The $3^{\text {rd }}$ visit, $4: 12 \mathrm{pm}$ )

Amy is in the sandpit. Ken is calling her. 'han wo gan shen ma?' (Why calling me?), murmurs Amy, as she walks out of the sandpit in response to Ken's call.

(The $1^{\text {st }}$ visit, $2: 45 \mathrm{pm}$ )

Amy moved to Ken and handed to him a book: 'Ken, xiang he wo kan zhe shu ma? (Do you want to read this book with me?)

(The $2^{\text {nd }}$ visit, 11:21am)

Amy comes to me: 'wo ma ma gei wo mai le yi jian mao yi (my mum bought me a jumper).

Karen: 'shi zhe jian ma (this one?)' pointing to the one on her.

Amy: 'bu shi. Zhi shi jiu de (no, this is an old one)'. (The $2^{\text {nd }}$ visit, 9:49am)

These examples indicate that Amy made appropriate choices for speaking Chinese. Never during my study did she speak it to the people who could not speak Mandarin, a phenomenon in tune with Baker and Jones's term (1998, p. 39) "language boundaries" referring to second language children's ability to associate each language with specific individuals or contexts.

\section{Speaking English to herself}

At times, Amy used English in her communication with herself. On one occasion I observed:

Amy walks to the animal box, getting down and picking up a little plastic 'elephant'. Nobody else is there. Amy speaks to the 'elephant': 'elephant, daxiang. Hello, elephant, daxiang, hello'.

$$
\text { (The } 5^{\text {th }} \text { visit, 9:26am) }
$$

It was a quiet self play situation. Using the 'elephant' as a prop, Amy was speaking to herself. An explanation for this could be that Amy was quietly practising English. Since 
it did not involve any other people, she did not need to decide which language to use, so that she spoke both.

\section{Speaking English and Chinese with Ken}

However, in the following observation, Amy used some English words in her conversation with Ken, a very close friend, who, as Amy described "does not know English" (Amy's interview) but understands Chinese quite well.

Amy holds up a book and calls up: Ken, come. Ni yao kan zhe ge ma? Hao da de yi ge da chuan, big' (do you want to read this book? A big ship is there, big). Ken comes, sits with her, reaching the book.

Amy removes Ken's hand: 'Ken, no touching, zhe shi wo de' (it's mine'). (The $3^{\text {rd }}$ visit, 3:02pm)

When telling me that "Ken does not know much English" Amy indicated her awareness that he might not have understood the English words, therefore it could be said that Amy did not make a correct language choice here. However, remembering SirajBlatchford and Clarke's (2001) idea regarding the importance of a stress free environment for second language young learners to practise their new language, perhaps Amy did not intend to engage Ken in dialogue but was simply practising her new language, after all, the 'no touching' phrase would have made little sense to him. Her use of 'no touching', therefore might have served the purpose of rehearsing the phrase because it was the only time I observed Amy using it and in view of her English ability, it might have been new to her. Amy might have perceived Ken's role as similar to that of the 'elephant'. Because neither Ken nor the 'elephant' understood English, they could not make a judgment on her use of the new language, and so she felt confident to use it with them. Amy's speech here therefore functioned as a self talk. However, what should not be neglected is her body language when she removed Ken's hand from the book. Here, Amy might have been demonstrating an awareness of the social underpinning of her speech and was attempting to ensure Ken understood what she said.

In these examples, Amy mixed the two languages, using both Chinese and English, especially in her conversation with Ken when she substituted words in Chinese with English. This occurred in Baker and Jones (1998, p. 37) as "code switching", a common feature of second language learning, and a result of interaction in two different 
languages. For young children, code switching could happen in a practical and purposeful way to express themselves with the available vocabulary. This would explain why Amy switched the languages in these two examples; she was purposefully practising the new language.

\section{Speaking English with teachers}

Amy also chose to use English with her teachers and she was able to take turns in her conversations with them. In the following example, Amy made conversational exchanges with her teacher Fiona.

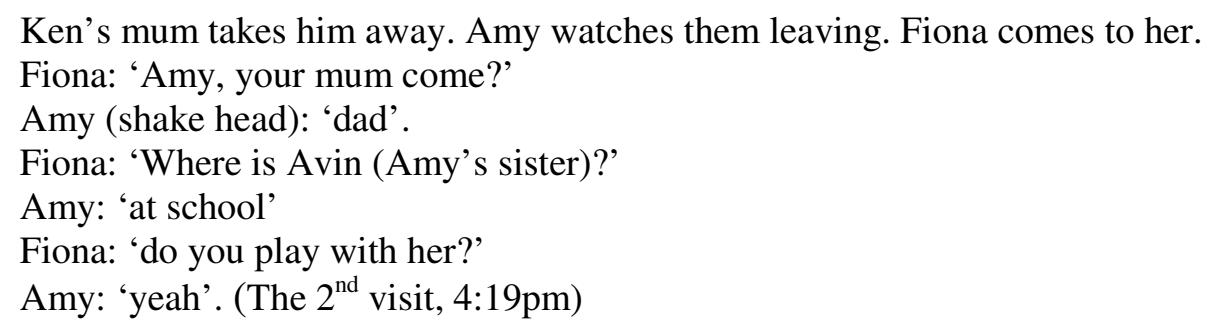

Following this conversation, Fiona and Amy sat down and found a book to read together, during which there were further language exchanges. Amy spoke in a simple way and responded happily. During my field work, Amy had many interactions with the teachers, involving speaking English. In several cases, it appeared that she chose to communicate with the teachers in the hope of learning English with them. In the next part of the story, I recorded how Amy sought teachers' help with her learning of the new language.

\section{A keen learner of the English language}

\section{Learning with the teachers}

Most of Amy's conversations were either with her teachers or Ken. She was aware of this and told me she wanted to talk to teachers because "I need to learn English" (child interview), thus it is reasonable to infer that Amy was motivated to learn the new language. Children's motives to learn a new language drives them to adopt strategies to learn it (Siraj-Blatchford \& Clarke, 2001). Amy, as demonstrated in the following excerpt, actively related to her teacher Maria and in doing so, demonstrated an attempt to learn with her teacher. 
Amy jumps to Maria, giving her a cuddle, sitting on Maria's lap, and handing to her a book.

Maria: 'want me to read it?'

Amy: 'yes'.

Maria reads the book. When she is finished, Amy leaves Maria and comes back with a pile of books.

Maria: 'too many'.

Amy: 'no, no many' and handing one to Maria.

Maria reads.

Maria leaves when hearing a child crying. Amy goes with Maria and once Maria settles that child, Amy takes Maria back to the floor. (The $1^{\text {st }}$ visit, $1: 59 \mathrm{pm}$ )

Their interactions were disrupted a few times but when Fiona left, Amy either left with Fiona or waited. As soon as she saw Fiona, Amy brought Fiona back to her.

On another occasion, I recorded the following notes:

Amy walks to her teacher Fiona, pointing to her hair clip.

Fiona: 'oh, that's pretty' (raising her voice).

Amy: 'my sister got pink... I don't like pink'.

Fiona: 'what colour do you like then, red', pointing to Amy's hair clip.

Amy: 'white and green too'.

Fiona: 'that's good. I also like green'.

(The $2^{\text {nd }}$ visit, 9:13am)

As shown in these examples, Amy used single words or short English phrases. I also observed her using body language, specifically pointing to what was referred to, to express herself. The key strategy that emerged from Amy's data was her engagement with teachers in interactions. In her language interactions with the teachers, Amy used some specific strategies.

\section{Adopting learning and communication strategies}

The strategies Amy used are listening, repeating and asking questions. My field notes from the first visit record Amy's language exchange with Fiona:

Amy settles on the floor with her teacher Fiona. Fiona is reading her a book.

Fiona points to an object in the book: 'what colour?'

Amy: blue', speaking loudly.

Fiona: 'this one'

Amy: 'green'.

Fiona points to a pineapple: 'what's this?'

Amy shakes head.

Fiona: 'pineapple'.

Amy: 'pineapple', loudly then again, 'pineapple'.

Amy looks at the book: 'is this a peach?

Fiona: 'no, apricot'.

Amy: 'apricot'.

(The $1^{\text {st }}$ visit, 8:48am) 
The conversation stopped when Fiona went to attend to other children, but after she returned, Amy moved on her lap again and told her that her shoe had a circle. Fiona did not respond but Amy gave her a gentle nudge and repeated. Apart from listening, repeating and asking questions, there was spontaneous speech here too, because Amy initiated the conversation and treated Fiona as a conversational peer, in an equal language activity. This is a very positive strategy. Grosjean (1982) believes that children learn a new language with determination if they are keen to be engaged in activities using the new language.

The following field note once again indicates Amy's happy engagement with Fiona in a language-related activity.

Fiona reads a story to Amy and another girl, who are sitting on Fiona's lap. Amy looks at the pictures in the book and says:

Amy: 'one, two, three, four, five flowers', pointing to the picture.

The other girl copies her.

Amy giggles and then turns to Fiona: 'Fiona, one, two, three, four, five flowers, yes?'.

(The $1^{\text {st }}$ visit, 9:05am)

Amy explicitly initiated the language exchanges and her spontaneous speech was noticeable in this situation.

Amy imitated her teachers too. I captured her standing on a chair by the window and teacher Maria, telling her 'you be careful'. Amy did not reply but after Maria left she smiled and repeated 'you be careful'.

At group (mat) time, Amy kept herself noticeably focused on teacher Iby's introduction of 'shapes'.

Iby is holding a book of shapes and a box of blocks.

I: 'What shape is it?' pointing to a circle in the book

Some children: 'a circle'.

I: 'what around us is in a circle shape?'

Children look around. Amy looks around too.

A child: 'the table'

Amy: 'ball'

Teacher I moves the whiteboard to her and draws a circle on it.

I: 'Circle'

Some children copy: 'circle'.

Amy: 'circle'.

I: 'what shape is it?' pointing to a diamond in the book.

There is a pause. 
One child suddenly bursts: a diamond.

I: 'yes, a diamond.

Amy (Speaks quietly): 'diamond.

I draws a diamond on the white board.

I: 'Do you know this one?' holding up a triangle block.

Amy (calling out): 'a triangle'. Some children also say 'triangle'

(The $5^{\text {th }}$ visit, 10:04am)

On another group learning occasion, I recorded Amy placing herself in the front row, closely watching and listening to Mary, a teacher who was sitting facing children, telling them a story, and discussing it with them.

Mary asks: 'any news to share today?'

The children are silent

A boy stands up, following Mary's gesture to go over to her, moving to Mary and tells his news.

Amy watches the boy and Mary, listening. She murmurs: 'went to the zoo', copying the word used by Mary.

When the boy sits back, Mary says: 'let's stand up, having a stretch'

Mary stands up. Amy stands up.

(The $4^{\text {th }}$ visit, 11:47am)

As in small group situations, Amy's learning was obvious in these large group learning contexts. She similarly listened with concentration, repeated the words and initiated spontaneous responses.

In the following note, Amy was seen to pick up a learning moment by repeating a sentence.

Teacher Iby is talking to two boys about their fighting when Amy walks past.

Iby: 'Tell him, I don't like it', to a boy.

Amy stops (loudly): 'tell him, I don't like it', and runs away.

Amy says again as she is running out: 'I don't like it'.

Amy bumps into a child and gives the child a push: 'I don't like it'.

(The $5^{\text {th }}$ visit, $3: 46 \mathrm{pm}$ )

Amy's use of the phrase 'I don't like it' to the child whom she pushed was in context and demonstrated her interest in using the new language. Even so, my observations were that Amy focused this interest only on teachers. She appeared to have no desire to take part in language interactions with her peers or to learn from them, although conversations among peers were a very prominent feature of the centre. 


\section{Meaningful language input of the teachers}

At this centre, children had many free play opportunities, during which Amy was frequently engaged in her own explorations. The teachers monitored the environment and encouraged children to learn themselves or with teachers (teacher interview). On these occasions, the teachers interacted with Amy, when they were seen to make an effort to give her meaningful language input. Amy appeared to understand them well. Meaningful language input was central to Baker's (1996) description regarding supporting second language young children and during my study with Amy, I noted examples of the teachers getting down to her level, using props, talking slowly and using simple words. Quite interestingly, Amy's teacher Iby learnt the Chinese greeting 'good morning' and used it with Amy and Ken. This could have given Amy a message that it was acceptable to use Chinese, so that she used it to herself and with Ken.

\section{Being a Member of the Group}

\section{Following the centre routines with ease}

The centre provided children with established routine activities, with which Amy appeared very comfortable. In the following example, she was actively taking part in the tidy up experience.

Iby (Amy's teacher) (calling): 'tidy up time'.

Amy is running around outside and quickly jumps inside, bending down, picking up things on floor.

Iby, Mary and Fiona, three teachers are tidying up and some children are helping too. Amy calls to Iby, who is standing close to her: 'Iby, me tidy up' Iby: 'good girl'.

When the room is clear, Amy goes outside to continue helping up. (The $4^{\text {th }}$ visit, 11:36am)

She said in our interview that 'it's no good that teachers are unhappy' (child interview). Amy's attitudes might be derived from her awareness being a group member and of respecting teachers.

Apart from 'tidy up', Amy joined other children in routines, such as meal time, sleep, mat time and she took responsibility to clear her table after meals. After playing with puzzles, blocks or books, she put them back to the right places every time. One day, 
Amy went to the bathroom and found the toilet bowl was soiled. She brought the toilet paper and carefully cleaned the bowl.

\section{A Chinese peer as a base of peer relationships}

Everyone was aware that the Chinese boy Ken was Amy's best friend. Amy told me during the interview, "Ken wants to play with me. I play with Ken". Ken was two years and six months at the time of the study. On numerous occasions during my field work, especially on the first three visits, Amy was with Ken. They sometimes interacted but mostly were just alongside each other. A teacher said: "Amy plays a big sister role to Ken' (informal discussion).

Sometimes, Amy initiated the contact with Ken, for example,

Amy jumps up and down by the entrance when Ken comes in with his mum. In seeing Ken, Amy's face lightens up. She walks to Ken and holds his hands.

(The $1^{\text {st }}$ visit: 10:02am)

On some occasions, it was Ken who searched for Amy. Amy, in return responded to him promptly and willingly. One day, a photographer came to the centre to take photos of the children and left his tool case on the floor:

Amy and some other children are banging the photographer's case. They are laughing. Ken, stands in the middle of the room, calling up: 'Amy'. Amy quickly stops banging, turning around, standing up, going to Ken and taking Ken's hand.

(The $3^{\text {rd }}$ visit, 9:29am)

When talking about friends, Amy regarded Ken as her best friend. There is also evidence that Amy cared about Ken. On one occasion, Amy washed her hands, sat at the meal table waiting for the food. Ken was still washing hands. I observed:

Amy put a hand on the chair next to her. A girl came, wanting to take the chair. Amy says: 'Ken', holding the chair for Ken. The girl moves to another chair.

Ken comes out of the bathroom. Amy waves to him. Ken goes to sit by her.

(The $2^{\text {nd }}$ visit, 2:49pm)

I also noted Amy going to Ken when Ken cried after a teacher physically stopped him from throwing toys. Amy was apart from Ken at that time but ran to him the minute she heard him crying. Amy did not talk to Ken but she coaxed him to sit down and then she sat with him. 
Ken was more like a follower to Amy, so his presence did not distract Amy from playing with others. I recorded Amy interacting with other children alongside Ken:

Amy is crawling on the floor with Ken. An English speaking boy approaches them with a tiger puppet and 'roar...' to them, lifting the tiger up close to them. Amy stands up and jumps to him, shouting, 'tiger...', then jumping away, giggling. The boy approaches her again, 'roar.....'. Amy roars back and says "hi tiger.', jumping away cheerfully.

(The $1^{\text {st }}$ visit, 11:00am)

Although Amy appeared to be very responsive to Ken, on one occasion, I recorded her rejection of Ken's attempt to play with her.

Amy is drawing at a table.

Ken goes to her, takes hold of Amy's hand and pulls her towards the door.

Amy: 'Ken, ni yao gan shen ma (Ken, what do you want to do?)'

Ken: 'na li wan' (go there), pointing outside.

Amy: 'bu, wo yao hua hua' '(No, I want to do drawing)'.

Ken pulls Amy out.

Amy: 'bu. Wo bu qu' (no, I am not going).

Ken gives up and leaves Amy.

(The $3^{\text {rd }}$ visit, 2:49pm)

In this situation, Amy insisted on staying inside because she wanted to do her drawing, which means that while Amy liked playing with Ken, she was not passive in their relationship.

Iby said that 'although Amy plays mainly with Ken, when Ken is not around, she also plays with other children. My field notes also recorded scenarios when she related to other children without Ken. Below is an example of that.

Amy is at the sandpit, cooking. A girl walks in with a little tube shaped plastic bag containing flowing fluid. The girl sits in the sandpit and waves her bag up in the air. Amy moves to her, watching. Amy points to the bag: 'stuck' when the fluid stops in the bag. Another girl approaches them, holding some wrapping paper. This girl gives paper to another girl and instructs her to wrap her plastic bag. She also hands to Amy some paper. Amy takes the paper, wrapping some sand in it. Amy then gives the girl a nudge and says: 'I'm finished'. The girl gives her more paper and Amy carries on wrapping sand. (The $1^{\text {st }}$ visit: $2: 45 \mathrm{pm}$ )

Amy's parents believed that Amy did not have a strong need for friendship, but she did mention some children's names to them. Her teachers talked about Amy's emerging 
relationships with some children who were friendly and easy going. At the end of my study, Amy interacted with Ken less but was still seen to use him as a base to develop friendships with others. In doing so, Amy tried to support Ken into her play with other children. This according to Amy is that "I want to play with many other children". It is possible to infer from these excerpts that Amy needed friends, but she was searching for friendships with children who looked friendly to her, and her relationship with Ken possibly served as a basis for her to develop further peer relationships. Because Ken was around, Amy was not desperate for friends and she avoided interacting with those who did not appear friendly to her. In the following note, Amy appeared restraining herself from interacting with some English-speaking peers.

\footnotetext{
Amy is drawing on a sheet of paper alongside three English-speaking girls. She is sitting on one side of the table next to Iby and the three English-speaking girls are sitting next to each other on the other side of the table. The English-speaking girls are drawing and they sometimes giggle among themselves. Amy colours a picture all red while the other children use a range of colours to draw. Amy stops, looking into the pen holder which is empty as the other pens have taken out by the girls and placed next to them.

Iby: 'finished?.

Amy: 'no', shaking head.

Iby: 'then you need to tell them you need pens'.

Amy makes no move. Iby gets a couple of pens from the other girls and hands them to Amy. Amy continues drawing. (The $2^{\text {nd }}$ visit, 2:03pm)
}

It was obvious that Amy wanted the pens, but she did not approach the other girls for them, perhaps because, as she told me after the incident, 'I dare not ask'. Here, Amy indicated, that she was not confident to approach the other girls because, as I observed, they did not look friendly to Amy and Amy might have felt the same. Amy might have felt that her approach to the girls would not be met with friendly compliance and she would risk rejection. Also, with Iby around, Amy relied on her support. I don't interpret Amy's language to be a limitation here because while her English was not proficient, she had enough language to handle the situation.

\section{Enjoying interactions with teachers}

My field notes include strong evidence illustrating how easily Amy related to the teachers, and an interview comment confirms teachers acknowledged this too. Amy seemed to respond to teachers with confidence. 
On my first day, a picture of how Amy related to Fiona is recorded in the following scenario. Here is how Amy greeted Fiona.

Amy is scribbling on the paint easel. She puts her head up when hearing the door open. Teacher Fiona arrives.

A: 'Hi, Fiona', calling loudly with excitement. A quickly drops her brush on the easel sill, runs to Fiona with her arms widely open. There is a large smile on A's face. Fiona gets down to Amy, holds her up and gives Amy a kiss on the cheek.

F: 'how are you, Amy?, watching Amy's eyes'

A: 'hi, Fiona', putting her head towards Fiona's chest, fiddling a button on Fiona's jacket and smiles.

(The $1^{\text {st }}$ visit, $8: 30 \mathrm{am}$ )

Amy's greeting to Fiona brought them together for an hour and twenty minutes, and although Fiona included other children in play, Amy was determined to be with her.

I recorded another example of Amy's interaction with her teacher Maria:

Amy goes outside (after sleep). She sees Maria and walks to her. Maria is standing by the playhouse, talking with a teacher in the four year group. Maria sees Amy, smiles to her and stretches her hand to Amy. Amy walks up and holds Maria's hand.

(The $5^{\text {th }}$ visit, 2:08pm)

\section{Joining teachers with peers}

While Amy did not seem to mind playing alone, she accepted a teacher's request for joining small group play organised by her teacher Mary.

Amy is walking around, holding a teddy bear in her arms. Mary and two children are sitting at a table threading pads that had many holes on.

Mary sees Amy: 'Amy, you want to come?'

Amy quickly runs to her and finds a chair to sit on.

(The $3^{\text {rd }}$ visit, $2: 19 \mathrm{pm}$ )

In many situations, I noted Amy happily accepting teachers' invitations to join peer learning opportunities.. In a teacher's presence, she appeared engaged in the learning opportunities with peers.

In addition, the teachers in Amy's centre seemed to try hard with their teaching. During the interactions with children, including large group situations, the teachers used a range of teaching resources. The manager told me "we are trying to make learning fun for the children here". It could also be concluded that Amy loved being with teachers because they were fun. 


\section{Helping teachers deal with peer behaviours.}

Amy was particularly responsive to teachers when requested to support them to manage other children's behaviors. I recorded:

Amy walks inside. Iby is telling a boy: 'Danny, take the ball out', seeing the boy bringing a ball in. The boy does not listen. Iby sees Amy: 'Amy, ask Danny to take the ball out'. Amy walks up to Danny: 'Danny, outside. Take it out'. The boy holds the ball with him. Amy snatches it and throws the ball out.

(The $1^{\text {st }}$ visit: $3: 23 \mathrm{pm}$ )

Similarly, she helped Maria deal with Ken's behavior. I recorded:

Ken is throwing toys around. Amy is playing on her own. Maria calls: 'Amy can you go to stop Ken?. He is throwing toys'.

Amy walks to Ken, grabs his hand, watching him and says: 'no. no. bu ke yi (don't do it)', speaking firmly.

(The $3^{\text {rd }}$ visit, 2:55pm)

Amy's parents attributed Amy's respectful attitudes to teachers to her childcare experience in China.

Mum: Amy is very friendly and many teachers, including the teachers in China like her. In China, children are scared of teachers and Amy knew that teachers needed to be respected. Teachers are teachers. Amy knows the importance of teachers to teach. The teachers there treated her well because she listened to them. Amy could be stubborn at times but she always behaves well. I just guess but do not know much about her relationships with the teachers here. In the last centre, the teachers were quite strict. Amy could not understand them and she was very unhappy. She told me once that she liked the teachers at this centre because they were good. So I think..en...Amy relates well with the teachers here. She knows the importance of teachers to teach.

Dad: Amy is always with adults. She is very close to her mum and knows how to interact with adults. She has no friend and may have difficulty to be with children, but adults should not be a problem.

\section{(Parent interview)}

These answers give further accounts to Amy's positive relationships with teachers. Apart from her desire to have somebody with her, Amy's affection for teachers is derived from her intention to learn from them and her learning to respect and to gain acceptance from teachers. This highlights the influence of Chinese culture on Amy, because she treated teachers with respect as teachers provide knowledge and stand for authority (Chao, 1995). Amy's childcare experience in China had laid a foundation for her to learn to relate to teachers. 


\title{
Amy's parents' viewpoints
}

Amy's parents reported that Amy loved reading, and she liked singing and dancing too. They were very clear that "Amy is Chinese and we should show her all the important aspects of Chinese traditions. The parents said they purchased Chinese cartoons for Amy and her sister to teach them the importance of hard working. They told the sisters Chinese stories whereby introducing them the valuable aspects of Chinese culture. The parents also said that it is of particular importance that Amy keeps her own language. "Although it is crucial that she picks up the new language, she must have Chinese as her home language" (Amy's mother). Her parents agreed that the beauty of Chinese culture rested on its emphasis on competition and academic learning. They had similar perceptions that a young child, like Amy should be introduced to academic writing and reading as the key learning elements alongside the development of good habits and a strong desire for learning.

\begin{abstract}
Amy's mother: her father is always worried about her [Amy]'s academic learning and always wants her to learn reading and writing. I teach Amy words and numbers. She learns both English and Chinese with us. Her dad teaches her science sometimes and her sister teaches her English and maths. Her sister has lots of after school classes and I sometimes take Amy along. She goes into the Chinese and music classes and sits there, but because she is still too little, she just stays there to listen. Her sister loves singing and Amy learns lots of English songs from her.
\end{abstract}

In addition, her mother explained: 'three-year-olds in China know a lot and the children here are much behind. I don't want Amy to be too much disadvantaged because she may go to China for primary school and it is important that we laid good academic foundations for her".

Except for some self help skills, Amy's mother believed that: "Amy has not learnt much at the centre. Her English is slowly progressing. I went to see the manager about having Amy moved to the 4-year-old group because Amy needed advanced learning, but the manager did not help. I just gave up, because it is pointless to go further".

Amy's parents wanted "learning should be more structured and more teaching and teachers should communicate with parents very often'. Amy's father added that "we don't know what Amy learns. I sometimes ask her what she learns and how many English words she has learnt. She knows she needs to learn the language because her 
language is different". The mother felt sad that Amy did not want them to go to the centre to see her and recalled Amy being upset when she visited her during the session for her.

The parents also admitted that they had not been invited to parent meetings except for the twenty minutes induction when Amy first joined the centre. Because of this, the parents said they did not know of the programs and they thought it was meaningless to ask because "the teachers tell nothing but only how good Amy is. My requests could only be a fuss to them. We think teachers should give us useful information and organise individual meetings once for a while". Amy's father states: "teachers are more important than parents [to children], as parents are too busy after work with housework and do not have time for children. I think it is important that Amy gets to know how to function in a Kiwi cultural environment. It is important that teachers help her develop the skills about this. Good teachers should be active and interact meaningfully with children. Amy's mother said: "I don't' like teachers not to organise the work but talk too much among themselves". Even so, at the end of the interview, Amy's parents said that this centre was good as Amy was happy. For them, "nothing else is more important than seeing Amy happy there. There are problems and we are not happy with the teachers but since Amy's happy, we can just let her go". The mother could not predict any problems with Amy's relationships with her teachers because "Amy knows the importance of teachers to teach".

During my field work, Amy's father came to the centre to pick her up and drop her off, and never during these visits did he communicate with teachers, but was always seen to be in a hurry. Although parent and teacher communications were not a clear feature in Amy's centre, some parents interacted with teachers.

The following note was taken in the morning of my first day of observation of Amy:

Amy's father takes Amy to the entrance and stops at the recording book, writing down the time of Amy's arrival. Amy walks into the room by herself. Her father waves 'goodbye' over the door. Amy: 'zai jian (bye). On his way out, Amy's father meets the centre manager, Mary, and smiles to her. Mary smiles back. 


\section{Amy's teachers' viewpoint}

Amy's teachers could not identify any features that suggested that Amy was different from local children. "Only when she first joined, we felt a bit at a loss at how to take off her layers and layers of clothes seeing her too hot. It is typical of many Chinese children we have had at the centre. Amy can easily concentrate on learning and have excellent small motor skills. She likes dancing. I guess she might have learnt to use her hands very early on" (teacher interview).

Amy's manager Mary told me that immigrant children were not really different but their parents' worried too much and their apprehension somehow gave their children a message that they were different, which meant the children were unhappy and unsettled. The centre manager mentioned Amy's mother's request regarding moving Amy to the four year old group. Her teacher Iby responded "as if the parents think that the children do not learn anything here". Mary believed that they should have explored some strategies to inform the parents of their practice and philosophies. During my visits, the teachers did not request information from Amy's parents and at most, they smiled to her father when he arrived. It is, therefore, clear that the parents were not informed, and the teachers knew little about Amy's family life. Mary said: "I have to admit that we do not really know much about these [Chinese] children's families". That is why she only guessed that Amy might have used her hands very early on. The teachers, including Mary all attributed the communication gap with Amy's parents to their difficult communication with them. . They also mentioned their concern regarding inappropriate communication with the parents. Therefore, they concentrated on the child. Iby said "our programme is child-centred. It's important that we pay attention to these [Chinese immigrant] children to help them settle". In her guide for professionals working with diverse cultural children, Keats (1997, p. 56) confirms that 'many [professionals] prefer to be able to deal only with the child, finding the parent from another culture difficult to understand".

Regarding working with Amy, the most evident strategy the teachers adopted was speaking with her. They all spoke slowly and clearly with her and taught her specific words associated with what was happening and concrete objects in the immediate environment. Related to their language support was also the teachers' practice of using 
Amy as a bilingual help. It was noticeable that when Maria requested her for support with Ken's behaviors, she conveyed to Amy a message that it was wonderful to know two languages, thereby encouraging her use of both languages in the centre.

\section{Researcher's reflection}

The information collected from all the participants is consistently agreed that Amy was happy and was developing in this new environment.

However, there were different perceptions and expectations for Amy's development. Her parents thought Amy was not developing as fast as she could and needed more advanced opportunities, whereas the teachers considered Amy was a typical child whose development was acceptable. Amy herself indicated a desire to learn more, therefore for her, she needed to relate to teachers because teachers could teach her.

As the researcher, I perceived Amy's experience to be very positive, however she should be provided with more learning opportunities based on her strengths and interests, such as music, and also she could be supported to develop appropriate ways to function in her learning context, for example building confidence to relate to more English-speaking peers.

There is definitely a concern about parents and teachers' communication. In Amy's case, her parents spoke English well and were confident to talk with teachers. They were disappointed with teachers' lack of information about their child and their difficulty to persuade teachers to change their practice as they wished. So, Amy's parents did not have the desire to relate to teachers. The teachers, however, anticipated communication difficulties and the parents' excessive demands to have restrained them from working with Chinese immigrant parents.

The ideas, values and practices of each of the participants in Amy's study are clearly important if one wants to understand Amy's experience in a New Zealand early childhood centre. I found Amy's attitude towards learning particularly helpful, because she was a determined learner, who perceived her centre experience to be a commitment but not a choice. The attitude is very powerful; it influenced Amy's use of Chinese, her 
development of English, and her relationships with teachers and peers. In an attempt to settle in an English-speaking setting, Amy tried to use the Chinese cultural tools she knew well to process the new cultural information, to relate to her Chinese peer, and she communicated in English with her teachers to learn from them. Amy chose to play with friendly English-speaking peers because they helped her develop group membership. Similarly, Amy minimised her interactions with the peers who appeared not to include her, to avoid potentially troublesome issues, which would contribute to difficulties of her settling in the environment. Amy's learning experience, as such, indicates her active agency in her own learning and development.

Amy's parents' Chinese cultural beliefs that academic development and structured learning are essential influenced their expectations of Amy's experience in the centre. I argue that this style of parents' values is important, because this perspective led to the parents perceiving teachers' work to be inappropriate, Amy's own commitment as important and their involvement in home-teaching Amy as necessary. This value would have also have contributed to the parents' limited participation in Amy's centre experiences.

In my opinion, the teachers in Amy's centre incorporated very little of Amy's home cultural resources. I tend to think of Iby's practice of a Chinese greeting as very basic and Amy's use of Chinese to be only an effortless approval of her teachers. When the teachers adopted a child-centred free play approach to teach Amy in the same way as they do to all the other children and made few attempts to work with Amy's parents, they mainly relied on the dominant mainstream cultural practices for Amy. Fortunately, despite her parents' disappointment with Amy's 'learning' at the centre, they considered Amy's happiness to be important. For this reason, the parents allowed the practices of the teachers.

I predict happier days for Amy at this centre, with her progressing to learn more English that may contribute to further friendships. At the same time, I also hypothesise her confusion about how to juggle the differences between home and the childcare setting. If the communication does not occur between her parents and teachers, Amy may be the one who needs to bridge the difference. 